\title{
Variation of fruit quality traits in apricot as sources for nutraceutical breeding
}

\author{
PhD THESIS
}

HELENA GÓMEZ MARTÍNEZ

València, October 2021

PhD. Supervisors:

María Luisa Badenes Català Elena Zuriaga García

UPV Supervisor: Santiago Vilanova Navarro 



\section{Agradecimientos}

Este trabajo ha sido posible gracias a la ayuda predoctoral FPI-INIA-2015, del Instituto Nacional de Investigación y Tecnología Agraria y Alimentaria (INIA), bajo el proyecto FPI2015-0042 con la denominación 'CPD2015-0245 Mejora sostenible de albaricoquero y melocotonero' y desarrollado en el Instituto Valenciano de Investigaciones Agrarias (IVIA), dependiente de la Generalitat Valenciana.

El trayecto a recorrer durante la realización de una Tesis Doctoral no siempre es un camino llano, por ello, me gustaría dar las gracias a todas a todas aquellas personas que de una u otra forma me han ayudado a que haya podido finalizar este trabajo y a que estos años hayan sido más fáciles.

En primer lugar, a mis directoras de tesis. A Elena por su ayuda y por lo que me ha enseñado durante este tiempo en el laboratorio, pero, sobre todo, por lo que he aprendido en lo personal. A Marisa me gustaría darle las gracias por la confianza depositada en mí y darme la oportunidad de incorporarme al grupo de Frutales del IVIA, pero también por ayudarme a buscar soluciones y darme su apoyo en tantos momentos de dificultad.

No quisiera olvidarme de mis compañeros de laboratorio del departamento de Frutales del IVIA: Gabino, Fany, Mati, Mar, Inma, Ana Conejero y Noelia, muchas gracias por ayudarme cuando lo he necesitado. También, quisiera recordar a Pepe y Julio, por sus consejos y, como no, también a Jose por sacar siempre tiempo y echarme un cable con la fruta. También quiero mencionar a Alba, Carles, Fran y Ángela, por lo bien que lo hemos pasado en "nuestro despachito", comidas y almuerzos y por esos momentos de esparcimiento a veces tan necesarios. Como no, me gustaría agradecer a Blasco su ayuda, sus consejos y apoyo en los momentos complicados, sabiéndome sacar una sonrisa. Ha sido una suerte coincidir contigo en el laboratorio, aunque haya sido por poco tiempo.

Quiero hacer mención al resto de compañeros del IVIA que me han prestado su apoyo. Almudena, gracias por tu ayuda con el HPLC y tus consejos, he aprendido mucho. A Miri y los "becarios de abajo", Estela y Carles, gracias.

Esta tesis también es fruto de mis primeros pasos entre laboratorios y, por ello, no puedo olvidarme de Manolo, Carmina, Amparo y Carlos. Gracias por seguir tendiéndome la mano a pesar de los años.

Durante la tesis, he pasado por momentos buenos y otros no tan buenos, por eso no puedo olvidarme de aquellos que siempre han estado a mi lado. Mari, Maïté, Carrión, Belén, Alba, Víctor y Raúl, gracias por hacer todo más llevadero y hacerme ver las cosas desde otra perspectiva.

Y, como no, también quisiera agradecer todo su apoyo a mis Mapalés, mi pequeña gran familia fallera, por preguntarme por "los higos, nísperos..., bueno, los albaricoques... eso que tú haces..." y por recordarme siempre que, pase lo que pase, somos Leones. 
Tampoco puedo olvidarme de mis balseños, especialmente de María e Irene. Gracias chicas por nuestras charlas (incluso a distancia), han hecho todo más ameno.

No obstante, si hay unas personas a las que verdaderamente tengo que dar las gracias es a mi familia, a mis tíos y primos, pero sobre todo esta tesis está dedicada a las personas más importantes de mi vida: mis abuelos y mis padres, ya que me han apoyado y ayudado a levantarme cuando yo sola no podía, me han aconsejado siempre y, en definitiva, soy lo que soy gracias a ellos. Gracias de corazón. 




\section{ABSTRACT}

Nowadays, there is a growing interest in a balanced and healthy diet, rich in fruit and vegetables with a positive effect on health and, particularly, with high antioxidant content. Simultaneously exists a consumer demand for high quality fruit with a balanced flavour and reminiscent of traditional varieties. As a result, breeding programmes are also aimed at improving the quality of the fruit, both in terms of the physicochemical properties of the fruit and its nutritional profile. From this point of view, the apricot is a complete fruit as far it is an extraordinary source of fibre, vitamins, phenolic compounds, and organic acids. Apricot properties make it possible to consume fruits both fresh and processed, which is exploited by the agri-food, pharmaceutical and cosmetic industries for the production of a wide variety of products.

At the Instituto Valenciano de Investigaciones Agrarias (IVIA) a breeding program is carrying out to obtain new apricot varieties but also to solve factors that directly affect production, such as selfcompatibility or resistance to Sharka, but also is aimed to improve fruit quality. As the first step in any breeding program, this work has identified sources of variation among the collection of accessions in the IVIA's apricot collection, detailing the composition profiles for the main sugars, organic acids, phenolic compounds, and vitamin C. In addition, a higher concentration of compounds has been identified in the peel than in the flesh, which makes this tissue a source of compounds of interest for the industry.

On the other hand, for a better knowledge of the genetic control of sugar metabolism, the expression of the key genes involved in this pathway (SUS, SPS and FK), related to QTLS previously described in soluble solids, was studied. In addition, the study was also supported with the corresponding phylogenetic analysis among different species, revealing the level of conservation among them and confirming the high level of synteny in the genus Prunus.

Regarding phenolic compounds, the expression of genes involved in critical points of the metabolic pathway (DRF, PAL and FLS) was analysed. Our results revealed that the red-blushed accessions are associated with a higher expression of ParDFR and ParPAL2, and the expression differences among paralogues could be due to the presence of a BOXCOREDLPAL, also related to genes involved in anthocyanin biosynthesis (ParDFR, ParFLS2 and ParPAL2). Simultaneously, the genetic effect in the offspring of 'Goldrich', one of the main genitors used at the breeding program for the introgression of Sharka resistance, was also studied. Results revealed that it improves the concentration of neochlorogenic and chlorogenic acids, as well as the genetic expression of ParPAL1. 


\section{RESUMEN}

Actualmente, hay un progresivo interés por una dieta equilibrada y saludable, rica en frutas y verduras con un efecto positivo en la salud $y$, particularmente, con alto contenido en antioxidantes. Paralelamente existe una demanda, por parte del consumidor, de fruta de alta calidad que tenga un sabor equilibrado y que recuerde a las variedades tradicionales. Todo ello ha propiciado que los programas de mejora genética también tengan como objetivo la mejora de la calidad de la fruta, tanto en propiedades fisicoquímicas del fruto como de su perfil nutricional. Desde este punto de vista, el albaricoque es un fruto muy completo ya que es una fuente extraordinaria de fibra, vitaminas, compuestos fenólicos y ácidos orgánicos. Sus propiedades hacen posible su consumo tanto en fresco o como procesado, lo que es aprovechado por las industrias agroalimentaria, farmacéutica y cosmética para la elaboración de una amplia variedad de productos.

En el Instituto Valenciano de Investigaciones Agrarias (IVIA) se está llevando a cabo un programa de mejora para la obtención de nuevas variedades de albaricoquero que, además de solucionar factores que afectan directamente a la producción, como son la autocompatibilidad o resistencia a Sharka, también tiene por objetivo la mejora de la calidad de la fruta. Como primer paso en todo programa de mejora, en este trabajo se han identificado fuentes de variación entre la colección de accesiones del banco de germoplasma del IVIA, quedando detallada la composición del perfil para los principales azúcares, ácidos orgánicos, compuestos fenólicos y de vitamina C. Además, se ha identificado una mayor concentración de compuestos en la piel del fruto que en su pulpa, lo que hace de este tejido una fuente de compuestos de interés para la industria.

Por otro lado, para un mejor conocimiento del control genético del metabolismo de azúcares, se estudió la expresión de los genes clave implicados en esta ruta (SUS, SPS y FK), relacionados con QTLS previamente descritos en materia de sólidos solubles. Además, también se reforzó el estudio con el correspondiente análisis filogenético entre diferentes especies, observándose el grado de conservación entre las mismas y confirmándose el alto grado de sintenia en el género Prunus.

Con relación a los compuestos fenólicos, se ha analizado la expresión de genes implicados en puntos críticos de la ruta metabólica ( $D R F, P A L$ y $F L S$ ). Nuestros resultados revelaron que las variedades más rojizas se asocian a una mayor expresión de ParDFR y ParPAL2, así como también que las diferencias de expresión entre parálogos podría deberse a la presencia de un BOXCOREDLPAL, relacionado también con los genes implicados en la síntesis de antocianinas (ParDFR, ParFLS2 y ParPAL2). Paralelamente, también se estudió el efecto genético que ejerce sobre su descendencia la variedad 'Goldrich', uno de los principales genitores empleados en el programa de mejora para la introgresión de la resistencia a Sharka, concluyendo que favorece la concentración de neoclorogénico y clorogénico, así como en la expresión génica de ParPAL1. 


\section{RESUM}

Actualment, hi ha un progressiu interés per una dieta equilibrada i saludable, rica en fruites i verdures amb un efecte positiu en la salut $i$, particularment, amb alt contingut en antioxidants. Paral-lelament existeix una demanda, per part del consumidor, de fruita d'alta qualitat que tinga un sabor equilibrat $\mathrm{i}$ que recorde a les varietats tradicionals. Tot això, ha propiciat que els programes de millora genètica també tinguen com a objectiu la millora de la qualitat de la fruita, tant en propietats fisicoquímiques del fruit com del seu perfil nutricional. Des d'aquest punt de vista, l'albercoc és un fruit molt complet ja que és una font extraordinària de fibra, vitamines, compostos fenòlics i àcids orgànics. Les seues propietats fan possible el seu consum tant en fresc com processat, sent també aprofitat per les indústries agroalimentària, farmacèutica i cosmètica per a l'elaboració d'una àmplia varietat de productes.

A I'Institut Valencià d'Investigacions Agràries (IVIA) s'està duent a terme un programa de millora per a I'obtenció de noves varietats d'albercoquer que, a més de solucionar factors que afecten directament la producció, com són l'autocompatibilitat o resistència a Sharka, també té per objectiu la millora de la qualitat de la fruita. Com a primer pas en qualsevol programa de millora, en aquest treball s'han identificat fonts de variació entre la col-lecció d'accessions del banc de germoplasma de l'IVIA, quedant detallada la composició del perfil per als principals sucres, àcids orgànics, compostos fenòlics i de vitamina C. A més, s'ha identificat una major concentració de compostos en la pell del fruit que en la seua polpa, convertint aquest teixit en una font de compostos d'interés per a la indústria.

D'altra banda, per a un millor coneixement del control genètic del metabolisme de sucres, es va estudiar l'expressió dels gens clau implicats en aquesta ruta (SUS, SPS i FK), relacionats amb QTLS prèviament descrits en matèria de sòlids solubles. A més, també es va reforçar l'estudi amb la corresponent anàlisi filogenètica entre diferents espècies, observant-se el grau de conservació entre les mateixes i confirmant-se l'alt grau de sintenia al gènere Prunus.

En relació amb els compostos fenòlics, s'ha analitzat l'expressió de gens implicats en punts crítics de la ruta metabòlica $(D R F, P A L$ i $F L S)$. Els nostres resultats van revelar que les varietats més vermelles s'associen a una major expressió de ParDFR i ParPAL2, així com també que les diferències d'expressió entre paràlogs podria deure's a la presència d'un BOXCOREDLPAL, relacionat també amb els gens implicats en la síntesi de antocianines (ParDFR, ParFLS2 i ParPAL2). Paral-lelament, també es va estudiar l'efecte genètic que exerceix sobre la seua descendència la varietat 'Goldrich', un dels principals genitors empleats al programa de millora per a la introgressió de la resistència a Sharka, concloent que afavoreix la concentració de neoclorogènic i clorogènic, així com en l'expressió gènica de ParPAL1. 


\section{ABBREVIATIONS}

\begin{tabular}{|c|c|}
\hline ANOVA & Analysis of Variance \\
\hline BHLH & Basic Helix-Loop-Helix domain \\
\hline BLAST & Basic Local Alignment Search Tool \\
\hline BLASTP & Basic Local Alignment Search Tool of Proteins \\
\hline BZIP & Basic Leucine Zipper domain \\
\hline cDNA & Complementary DNA \\
\hline DTT & DL-dithiothreitol \\
\hline DW & Dry Weight \\
\hline FK & Fructokinase \\
\hline FW & Fresh Weight \\
\hline HPLC & High-Performance Liquid Chromatography \\
\hline HPLC-DAD & High-Performance Liquid Chromatography with Diode-Array Detection \\
\hline LC-MS & Liquid Chromatography-Mass Spectrometry \\
\hline LG & Linkage Group \\
\hline MD locus & Maturity Date locus \\
\hline PC & Principal Component \\
\hline PCA & Principal Component Analysis \\
\hline PCR & Polymerase Chain Reaction \\
\hline PCR & Polymerase Chain Reaction \\
\hline PPV & Plum Pox Virus \\
\hline PVP-40 & Polyvinylpyrrolidone \\
\hline qRT-PCR & Quantitative Real Time RT-PCR \\
\hline QTL & Quantitative Trait Loci \\
\hline RNA & Ribonucleic Acid \\
\hline SDH & Sorbitol Dehydrogenase \\
\hline SI & Sweetness Index \\
\hline SPS & Sucrose-6-Phosphate-Synthase \\
\hline SSC & Soluble Solids Content \\
\hline SUS & Sucrose Synthase \\
\hline TSI & Total Sweetness Index \\
\hline
\end{tabular}




\section{INDEX}

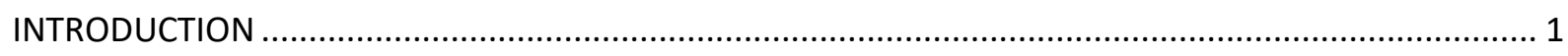

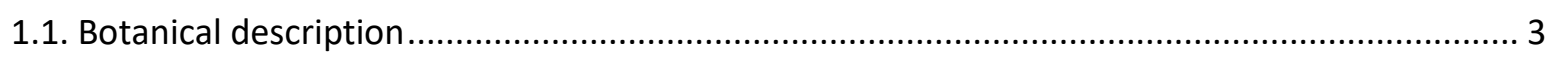

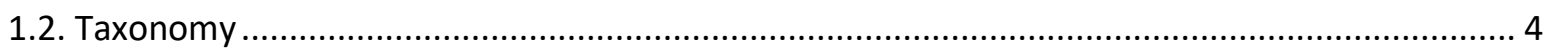

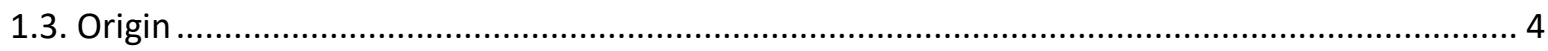

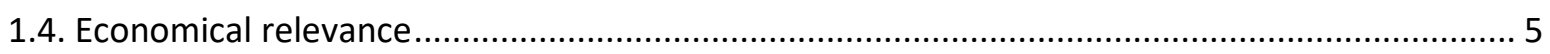

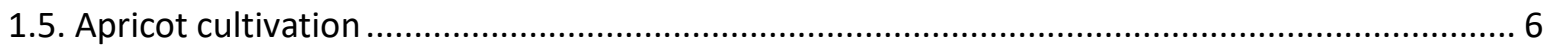

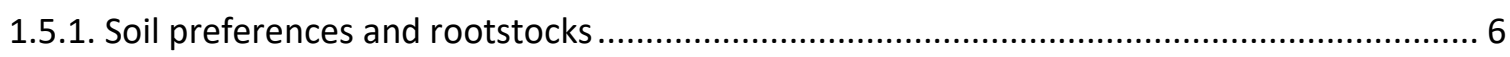

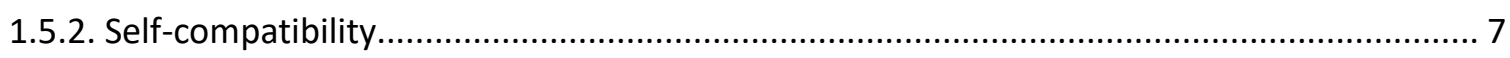

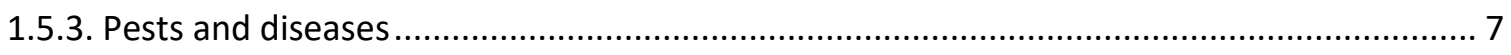

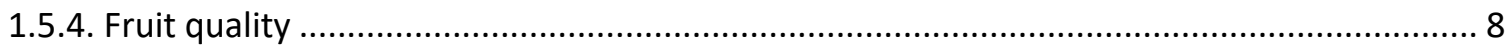

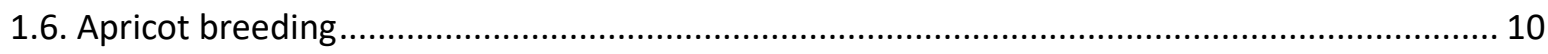

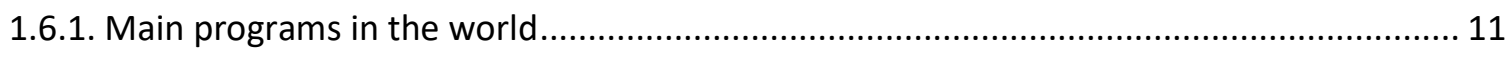

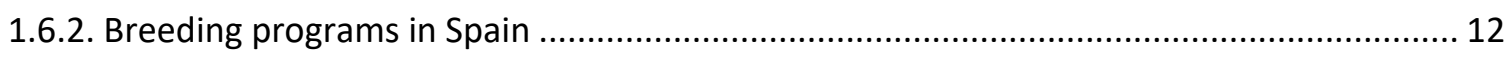

1.6.3. Germplasm and genetic resources for apricot breeding ............................................. 13

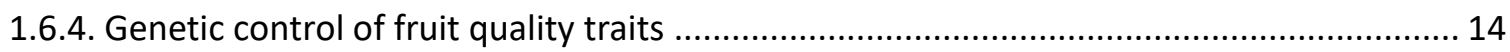

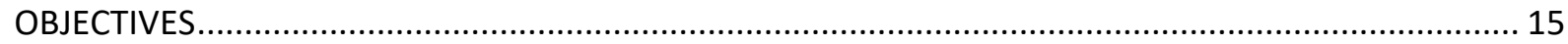

CHAPTER I: Nutraceutical profiles of apricots (Prunus armeniaca L.) as a source of fruit quality

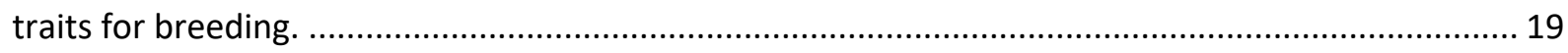

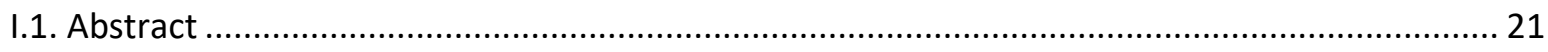

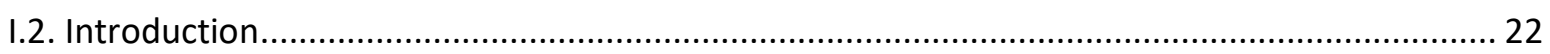

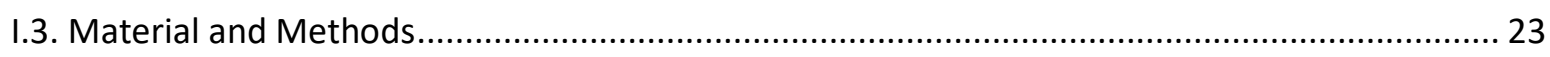

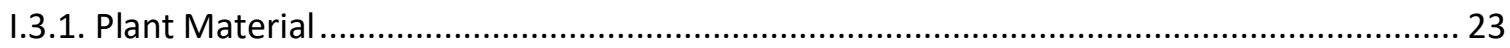

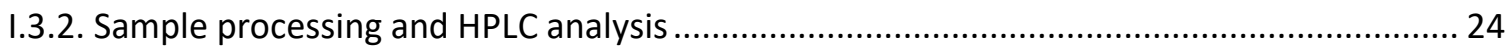

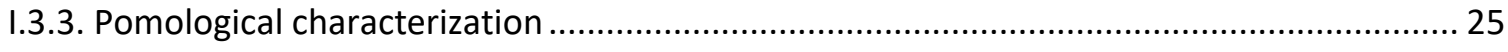

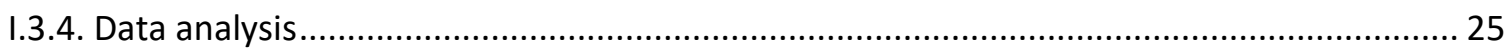

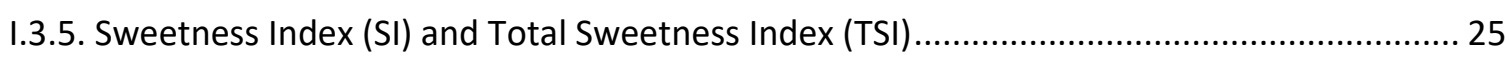

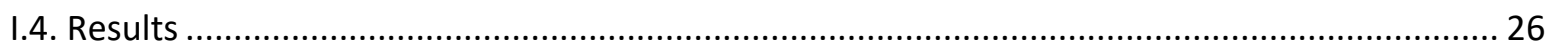


I.4.1. Sugars

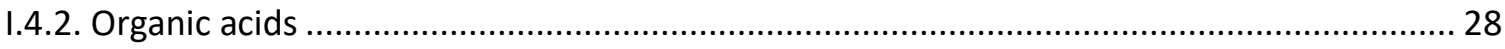

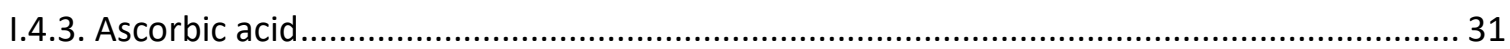

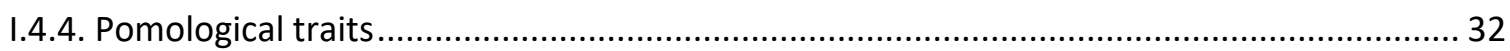

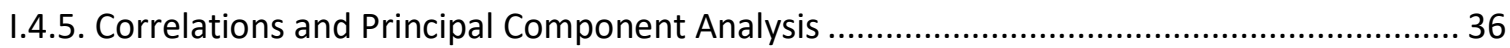

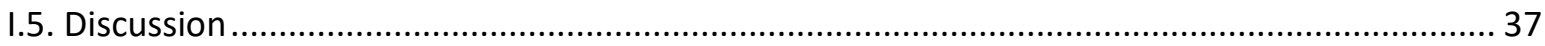

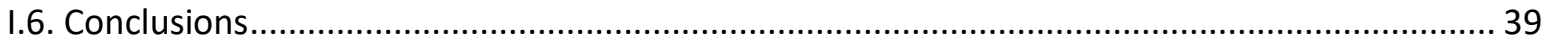

CHAPTER II: Sugar content and sugar-related gene expression in apricot fruits (Prunus

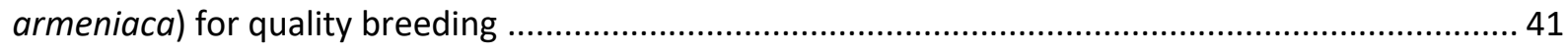

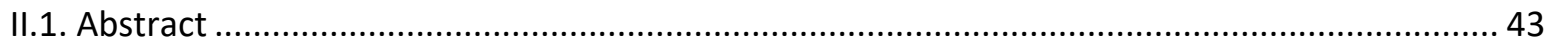

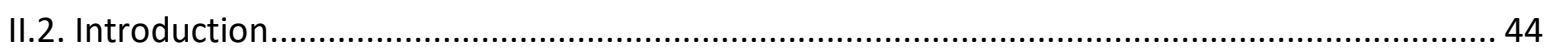

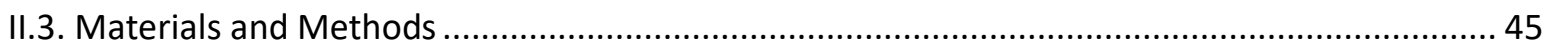

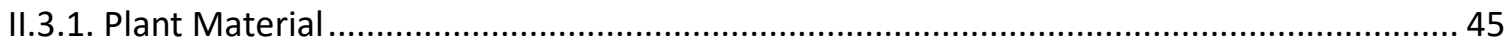

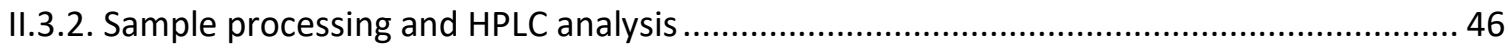

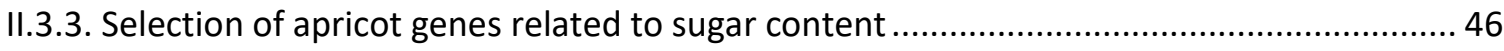

II.3.4. Phylogenetic analysis of sugar related genes.................................................... 46

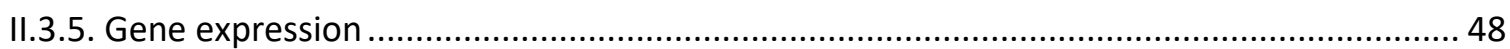

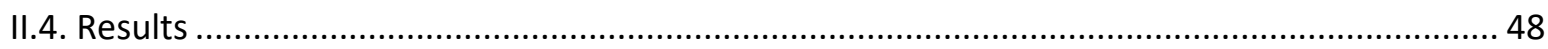

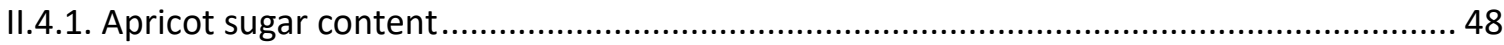

II.4.2. Sugar QTL-related genes selection and phylogenetic analysis.................................. 49

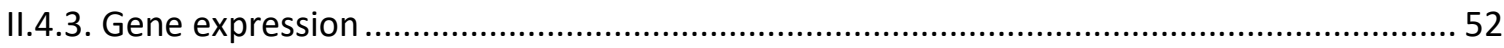

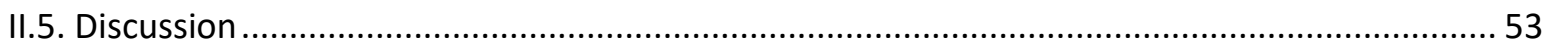

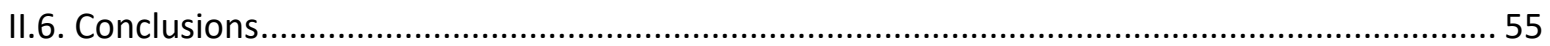

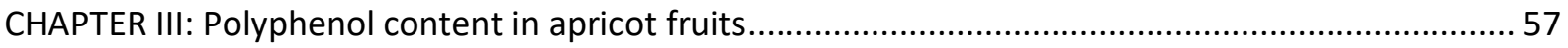

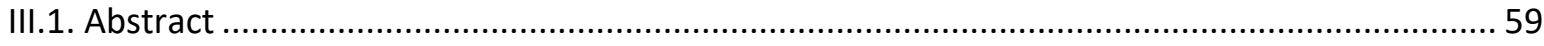

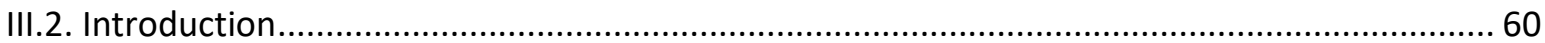

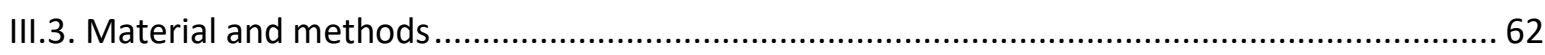

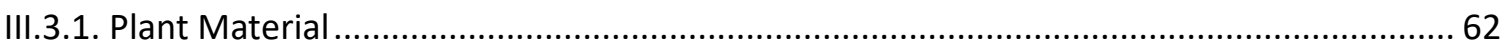


III.3.2. Extraction and HPLC of phenolic compounds

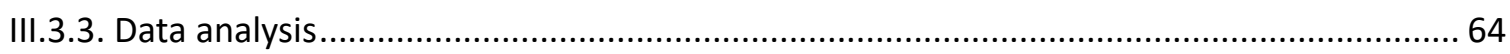

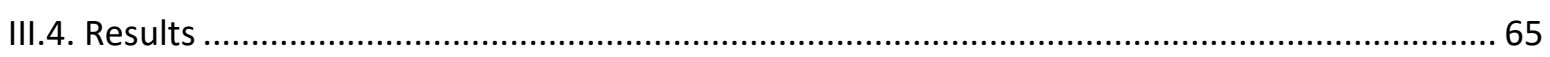

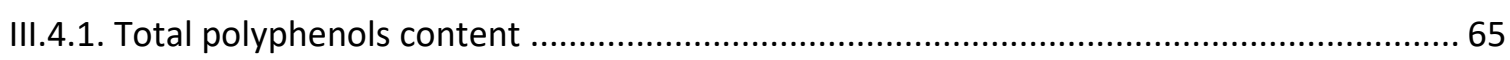

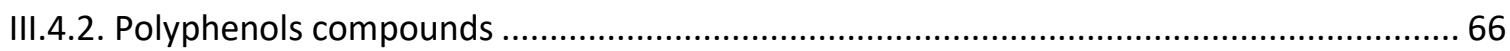

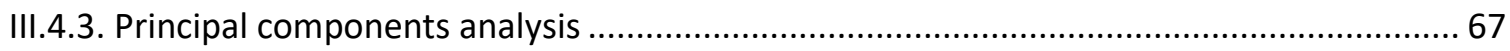

III.4.4. Contribution of the resistant cultivar 'Goldrich' to the quality traits studied. ................69

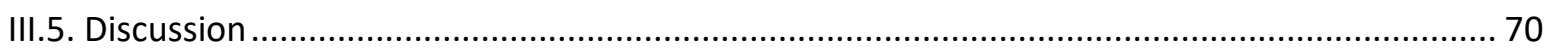

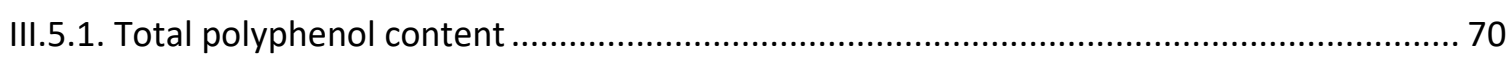

III.5.2. Contribution of the PPV-resistant 'Goldrich' variety to fruit quality ............................ 72

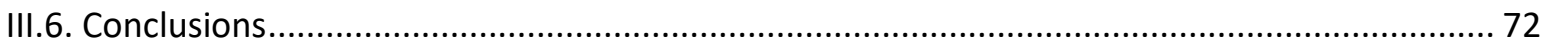

CHAPTER IV: Insights of phenolic pathway in fruits: transcriptional and metabolic profiling in

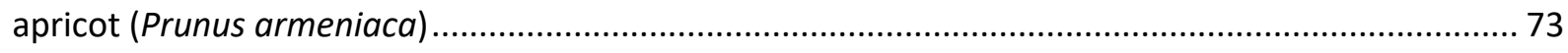

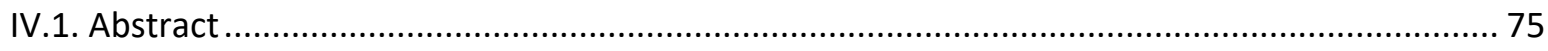

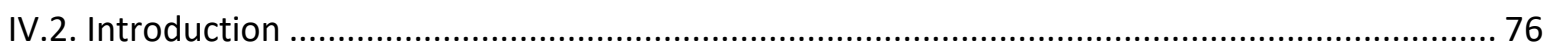

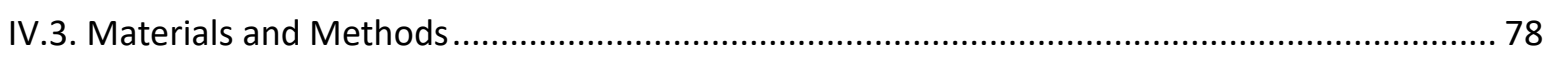

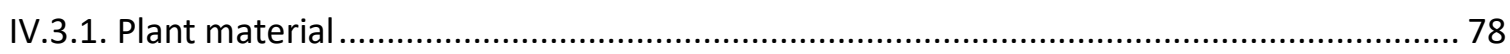

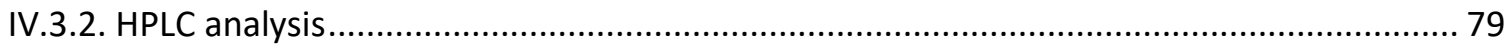

IV.3.3. Obtention of gene sequences and cis-acting elements motif identification .................. 79

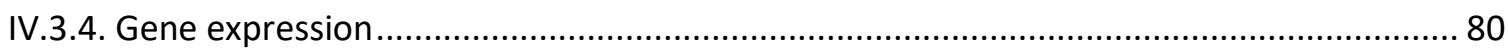

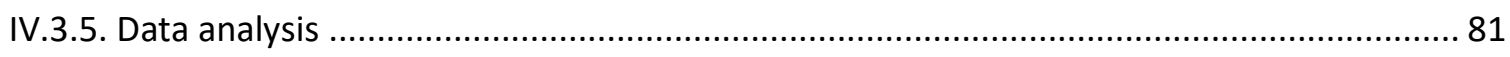

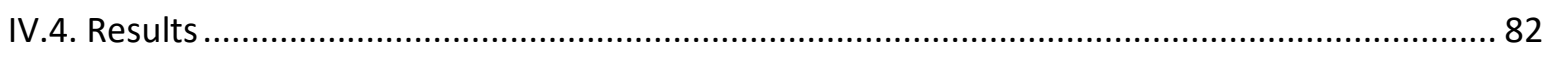

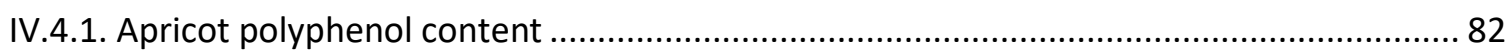

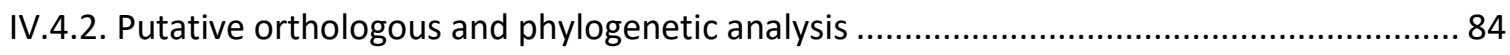

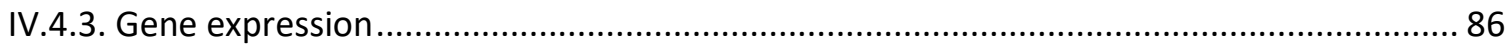

IV.4.4. Contribution of 'Goldrich' to phenolic compounds content and genetic expression........86

IV.4.5. Relationships between gene expression and phenolic compound accumulation............88

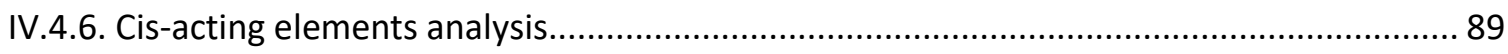




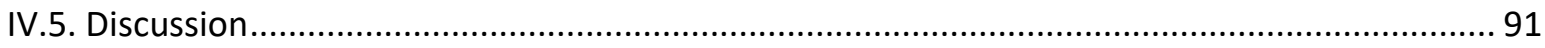

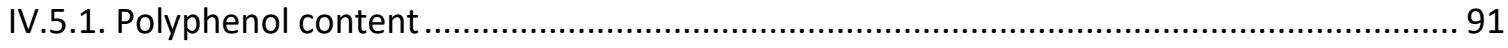

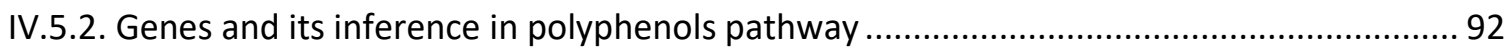

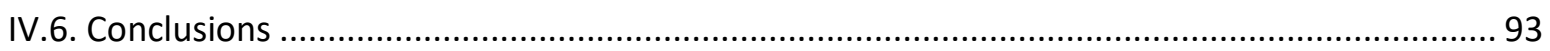

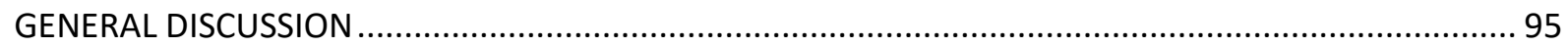

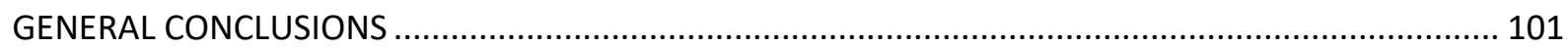

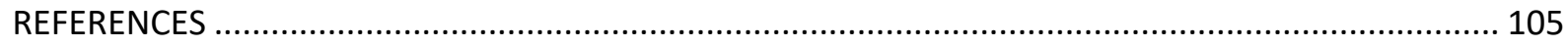

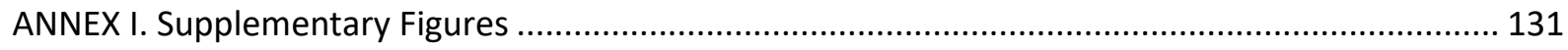

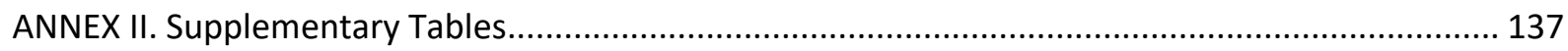

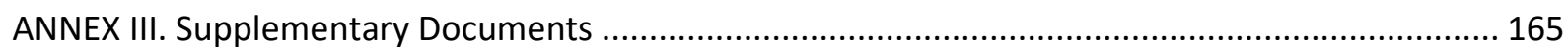




\section{FIGURE INDEX}

Figure 1. Details of apricot morphology: (A) Tree; (B) Leaves; (C) Buds; (D) Flowers.; (E) Fruit. Source: IVIA.

Figure 2. Total production (t) and cultivated area (ha) of apricot worldwide from 2009 to 2019

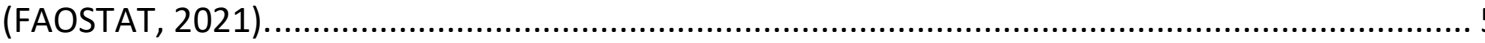

Figure 3. Worldwide and Mediterranean Basin apricot production (t) in 2019 (FAOSTAT, 2021)........ 6

Figure 4. Apricot PPV symptoms in leaves, fruits, and stone (Rubio et al., 2009). 8

Figure 5. PPV-resistant 'Dama Taronja' (A) and 'Dama Rosa' (B) apricot fruits (Badenes et al., 2018).

Scale bars in centimetres. 13

Figure 6. Significant correlations among variables analysed $(\alpha=0.05)$.

Figure 7. Principal Component Analysis for 2017 for pomological and nutraceutical data. Left: first and second components; Right: first and third components 36

Figure 8. Sugar content profile in the studied accessions (g/100g DW). 49

Figure 9. Neighbor-joining phylogenetic trees. A: SDH proteins; B: FK1 proteins; C: SUS proteins; D: SPS proteins. The percentage of replicate trees in which the associated taxa clustered together in the bootstrap test (1000 replicates) are shown next to the branches.

Figure 10. Gene expression of studied apricot genes.

Figure 11. Polyphenol compounds concentration: Neochlorogenic acid, chlorogenic acid and Rutin.

Data from 2016. A: Concentration in peel; B: Concentration in flesh.

Figure 12. Plot of the variables studied and accessions in the space defined by the two first PC. ...... 68

Figure 13. Phenolic biosynthesis pathway.

Figure 14. Examples of apricot fruits from Mediterranean varieties used as genitors in the breeding program with high redblush on the skin. This trait resulted related to anthocyanin content. A: Fruits from 'Ginesta'; B: Fruits from 'Palau'.

Figure 15. Chlorogenic (A), neochlorogenic (B), rutin (C) and quercetin-3-glucuronide (D) contents (mg/100g DW) in 2019 and 2020.

Figure 16. Neighbour-Joining phylogenetic tree for the proteins encoded by PAL (A), DFR (B) and FSL (C) genes. Data was bootstrapped 1000 times. Numbers close to each branch represents the percentage of replicate trees in which the associated taxa clustered together in the bootstrap test. Trees are drawn to scale according to evolutionary distances ( $p$-distance), included under each tree representing the number of substitutions per site.

Figure 17. Genetic expression (average of both years of study) of ParPAL1 (A), ParPAL2 (B), ParDFR (C) and ParFLS2 (D). Bars represent standard deviation. Different letters represent statistically significant differences. 
Figure 18. Significant correlations from the linear regression model for each compound.

Figure 19. Analysis of cis-acting elements on $1500 \mathrm{bp}$ upstream from start codon (ATG) sequences of Prunus armeniaca DFR, PAL and FLS genes. MRE: MYB-like Recognition Element; MYC: MYC-like recognition sequence; TATA: TATA box-like; BOXLCOREDCPAL: Consensus of the putative "core" sequences of box-L-like PAL promoter region. 90 


\section{TABLE INDEX}

Table 1. Taxonomic classification of Prunus armeniaca.

Table 2. Profiles of sugar content in flesh ( $\mathrm{g} / 100 \mathrm{~g}$ fresh weight (FW)) and peel $(\mathrm{g} / 100 \mathrm{~g}$

Dry Weight (DW)) during 2016, 2017 and 2019.

Table 3. Profiles of organic acids content in flesh (g/100 g FW) and peel (g /100 g DW)

during 2016, 2017 and 2019.

Table 4. Ascorbic acid content in flesh (mg/100 g FW) and peel (g /100 g DW) during 2016,

2017 and 2019.

Table 5. Pomological traits measured during 2016, 2017 and 2019.

Table 6. Plant material and sugar content (g/100g DW).

Table 7. Studied genes and synteny between Arabidopsis thaliana and Prunus persica and

Prunus armeniaca.

Table 8. Peach genes and sugar-related QTLs in Prunus persica.

Table 9. Plant material.

Table 10. Pomological traits measured in the genotypes studied related to fruit size and firmness. 3-years average \pm standard deviation. Different letter means significant differences among genotypes.

Table 11. Phenolic compounds: Neochlorogenic, chlorogenic, rutin and quercetin-3glucuronide. 3-years average \pm standard deviation. Different letter means significant differences among genotypes.

Table 12. Variable contribution to Principal Components, eigenvalues, and cumulative variance in the PCA. 68

Table 13. General Linear Model for phenolic compounds and pomological traits to test the 'Goldrich' effect and interaction. SSi: Sum of Squares; SS relative: SSi/SStotal; Year: environmental effect due to the year; G61: genetic main effect of 'Goldrich'; Year x G6161

Table 14. Variables studied and 'Goldrich' contribution. C: General average value of the

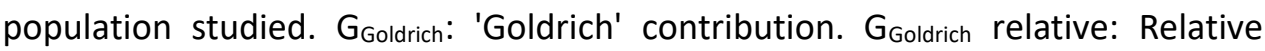
contribution of 'Goldrich' to the general average. Confidence intervals at 95\%. 70

Table 15. Plant material used in the study, pedigree, and origin 78 
Table 16. Used primers in the studied and reference genes amplification.

Table 17. Polyphenol total content (mg/100gDW). Average \pm standard deviation. Different letter means significant differences among genotypes. Varieties with * produced fruits with a redblush of skin $>50 \%$

Table 18. 'Goldrich' contribution to phenolic content: Sum of squares (SS) and model parameters coefficients. SSr: SS relative; SSt: SS total; P-v: P-value; Gr: 'Goldrich' relative; Sig: Significance.

Table 19. 'Goldrich' contribution to genetic expression: Sum of squares and model parameters coefficients. SSr: SS relative; SSt: SS total; Pv: P-value; Gr: 'Goldrich' relative; Sig: Significance. 88

Table 20. Pearson correlation coefficients among compounds and gene expression 88 


\section{SUPPLEMENTARY FIGURES INDEX}

Figure S1. Profiles of sugar content in flesh (g/100 g fresh weight (FW)) and peel (g /100 g dry weight (DW)) during 2016, 2017 and 2019. 133

Figure S2. Profiles of organic acids content in flesh (g/100 g FW) and peel (g /100 g DW) during 2016, 2017 and 2019.

Figure S3. Ascorbic acid content in flesh (mg/100 g FW) and peel (mg /100 g DW) during 2016, 2017 and 2019

\section{SUPPLEMENTARY TABLES INDEX}

Table S1. Spearman's rank correlation coefficients (upper triangular matrix) and significance levels (lower triangular matrix) among variables analyzed.

Table S2. Sweetness estimation, SI and TSI were calculated according to Magwaza and Opara (2015)

Table S3. Used primers in the qPCR amplification for sugar-related and reference genes 144

Table S4. Sugar content (g/100gDW) in the studied accessions in 2019 and 2020. 145

Table S5. Homologous proteins in other species which sugar content in fruit has economically importance in Prunus persica (A) and Prunus armeniaca (B). Ev: EValue

Table S6. Gene expression. Mean, standard deviation (sd) and significance (sg) 150

Table S7. Polyphenol total concentration in flesh (mg/100g FW) and peel (mg/100g DW) for each year and three years average (mean \pm standard deviation). Different letter means significant differences among genotypes

Table S8. Neochlorogenic acid concentration in peel (mg/100g DW) in 2016, 2017, 2018 and three years average. Mean \pm standard deviation. Different letter means significant differences.

Table S9. Chlorogenic acid concentration in peel (mg/100g DW) in 2016, 2017, 2018, and three years average. Mean \pm standard deviation. Different letter means significant.

Table S10. Rutin concentration in peel (mg/100g DW) in 2016, 2017, 2018, and three years average. Mean \pm standard deviation. Different letter means significant differences. 
Table S11. Quercetin-3-glucuronide content in peel (mg/100gDW) in 2016, 2017, 2018 and three years average. Mean \pm standard deviation. Different letter means significant differences.

Table S12. Prunus persica and Prunus armeniaca synteny and protein identity 153

Table S13. Arabidopsis thaliana and Prunus armeniaca protein identity. 154

Table S14. p-distance for PAL (A), DFR (B) and FLS (C) proteins. 155

Table S15. Similarity (1-p-distance) among protein sequences of PAL (A), DFR (B), FLS (C). 157

Table S16. Genetic expression of studied genotypes. Different letter means significant differences among genotypes. 159

Table S17. Linear regression model in caffeate-derivates. 160

Table S18. Linear regression model in flavonols. 162

\section{SUPPLEMENTARY DOCUMENTS INDEX}

Document S1. Acceptance mail of the publication "Nutraceutical profiles of apricots (Prunus armeniaca L.) as a source of fruit quality traits for breeding" (Chapter 1) in the Spanish Journal of Agricultural Research. 
INTRODUCTION 


\subsection{Botanical description}

Apricot (Prunus armeniaca L.) is a diploid species ( $2 n=2 x=16$ chromosomes), with a genome size of $\sim 220 \mathrm{Mb}$ (Jiang et al., 2019). Apricots are stone fruit trees adapted to temperate areas with a smallmedium size reaching 7-8 m high and dark bark (Agustí-Fonfría, 2010). Apricot trees (Figure 1) are deciduous and required chilling hours for overcoming the dormancy which varies among cultivars (Ruiz et al., 2006). Leaves are large, with a shape from elliptic to cordate, serrated margins, and long redpurple petioles (Agustí-Fonfría, 2010). Apricot flowers are hermaphroditic and appear from lateral buds, sprouting before leaves in late winter or spring (Agustí-Fonfría, 2010; Pérez-Pastor et al., 2004). Flowers are composed of 5 petals and sepals, with a high number of stamens and a single pistil with a carpel. Although two ovules are in the flower, only one evolves to seed after fecundation. Fruits consist of a drupe, with a hard endocarp that surrounds the seed, a fleshly mesocarp and a thin exocarp.

Apricots revealed a great diversity in pomological traits. The Community Plant Variety Office published a protocol for distinctness, uniformity and stability that reflects the different characteristics that can be used to discriminate between different cultivars (CPVO and EU, 2008). For instance, skin and flesh colour can vary from light cream to dark orange. Furthermore, apricots can present pubescence, a ventral suture and can present a blush that can surround the fruit completely. Moreover, the blush colour can differ from orange-reddish to purple. Seed shape is ovate, and stone can differ from circular to elliptic (Agustí-Fonfría, 2010).

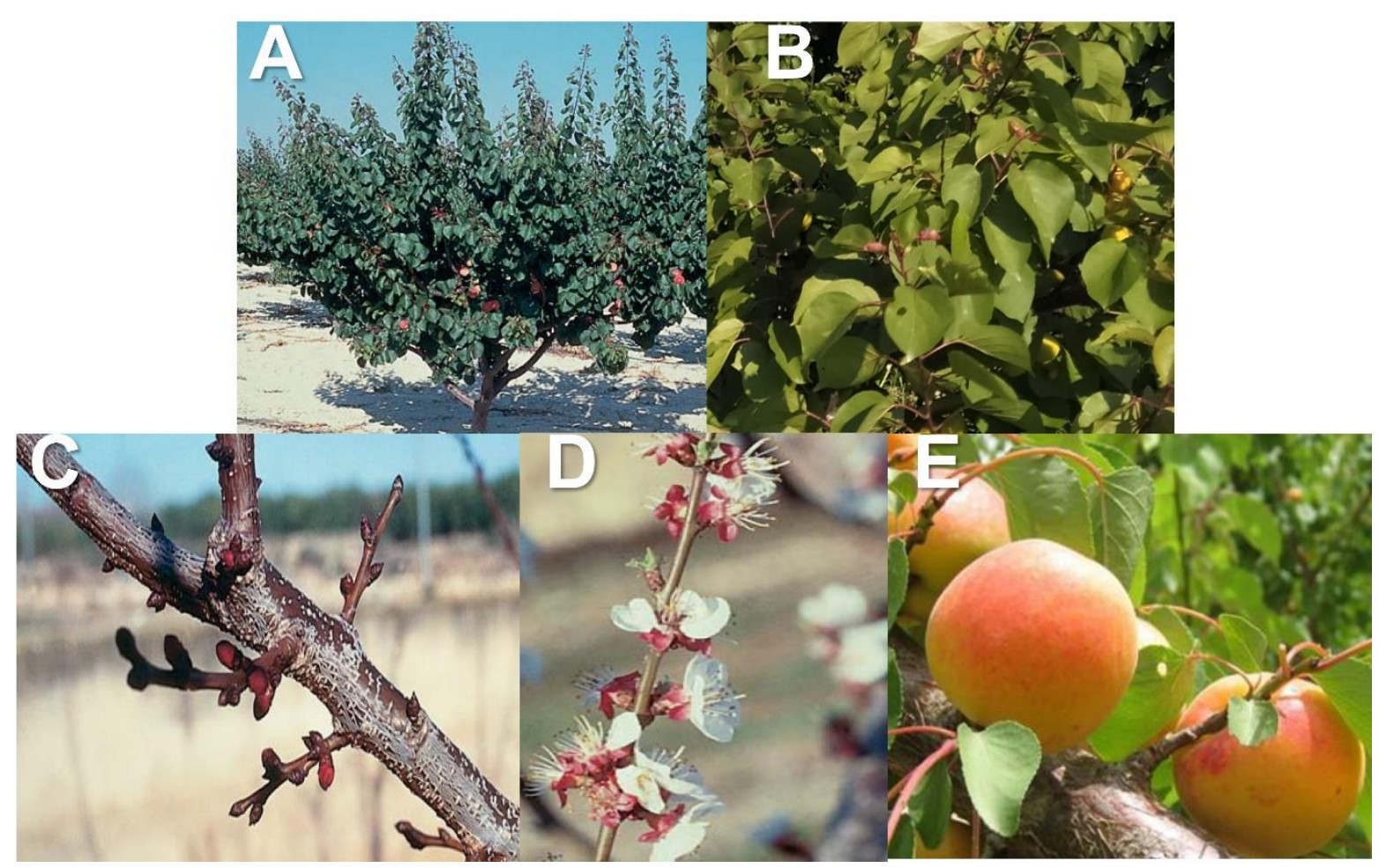

Figure 1. Details of apricot morphology: (A) Tree; (B) Leaves; (C) Buds; (D) Flowers.; (E) Fruit. Source: IVIA. 


\subsection{Taxonomy}

Apricot belongs to the Rosaceae family and is classified inside the tribe Amygdaleae, in the genus Prunus at the Armeniaca section (NCBI, 2021). The complete taxonomic classification of apricot (NCBI: txid36596) is described in Table 1. Depending on the classification system, the number of apricot species varies from 3 to 12 . From them, six are commonly recognized according to Zhebentyayeva et al. (2012): P. armeniaca L. is the common apricot, P. mume (Sieb.) Sieb. \& Succ. is the Japanese apricot, P. brigantina Vill. appears in the French and Italian Alps, P. mandshurica (Maxim.) is the Manchurian apricot, $P$. sibirica L. is the Siberian apricot, and P. holosericeae Batal. is the Tibetan apricot.

Table 1. Taxonomic classification of Prunus armeniaca.

$\begin{array}{lc}\begin{array}{l}\text { Superkingdom } \\ \text { Kingdom }\end{array} & \begin{array}{c}\text { Eukaryota } \\ \text { Viridiplantae } \\ \text { Phylum }\end{array} \\ \text { Subphylum } & \begin{array}{c}\text { Streptophyta } \\ \text { Streptophytina } \\ \text { Class }\end{array} \\ \text { Order } & \text { Ragnoliopsida } \\ \text { Family } & \text { Rosales } \\ \text { Subfamily } & \text { Amygdaloideae } \\ \text { Tribe } & \text { Amygdaleae } \\ \text { Genus } & \text { Prunus } \\ \text { Section } & \text { Armeniaca (Lam.) Koch } \\ \text { Species } & \text { Prunus armeniaca L. }\end{array}$

\subsection{Origin}

According to Vavilov (1951), apricots are native to China and Central Asia being the result of two successive domestication events. Following the Silk Road, the apricot culture arrived to the IranoCaucasian region, pointed as a secondary center of diversification (Vavilov, 1951). Later, based on morphological and physiological traits, four eco-geographical groups were recognised by Kostina (1964): Central Asian, Irano-Caucasian, European and Dzhungar-Zailij. Later, Layne (1996) added two new ones: North Chinese and East Chinese groups.

However, the history of apricot domestication remains unclear. As reviewed by Zhebentyayeva et al. (2012), Central Asia and China were considered as independent centers of domestication by most of the contemporary authors, but it was not clear which of the two regions was the first one. In a recent 
study using an important worldwide germplasm collection including 890 apricot accessions and 25 microsatellite markers (Bourguiba et al., 2020), the accessions from China and Central Asia clustered together and showed the highest level of genetic variability. According to these authors, apricots followed 3 different diffusion routes from the center of origin to the rest of the world (Bourguiba et al., 2020). First route was followed through Eastern Asia to Japan 2,000 years ago. Second route was from the Irano-Caucasian region through Mediterranean countries to Morocco. In previous works, two different routes were distinguished from the Irano-Caucasian region, one through the Southern European countries and the other through the North African ones (Bourguiba et al., 2012). Finally, the third route was through the Continental European countries.

More recently, a population genomics study using 600 apricot genomes pointed two independent domestication events from distinct wild Central Asian populations as the origin of Chinese and European apricots ca. 2,000-3,000 years ago (Groppi et al., 2021). According to these authors, Chinese cultivated apricots had higher genetic diversity than the European ones, that could be explained by their lower fraction of self-compatible accessions or/and the gene flow with wild relatives. Knowing the geographic areas where there is a greater genetic diversity is very useful for the search for variability in traits of interest for breeding purposes.

\subsection{Economical relevance}

Apricot, an important fruit tree in the temperate areas of both hemispheres, is highly appreciated for its edible fruits. The different qualities of apricot are used by the agri-food industry since it can be consumed both fresh and dry, what enables apricots to be used for the preparation of nectars, juices, jams, sweets, or desserts. In addition, apricot kernel oil is also used in perfumery, cosmetics, and pharmaceuticals (Xi and Lei, 2020). In addition, apricot fruits are highly appreciated by consumers because of the great health benefits associated with their organoleptic and nutraceutic properties.

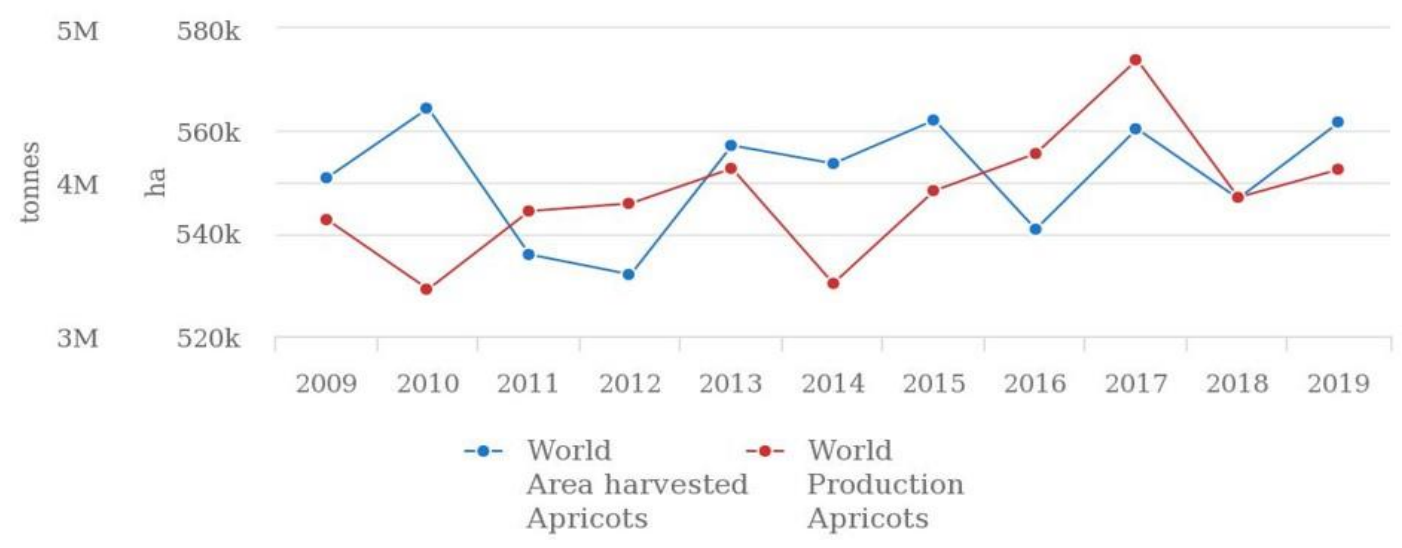

Figure 2. Total production ( $t$ ) and cultivated area (ha) of apricot worldwide from 2009 to 2019 (FAOSTAT, 2021). 
In 2019, more than 561,750 ha and more than 4 million tonnes were harvested (Figure 2). Asia accounts for the $60.7 \%$ of the worldwide production and Europe for the $24.5 \%$ (Figure 3 ). The main countries producing apricot are located in the Mediterranean basin, being Turkey the main apricot producer with 846,606 tonnes, followed by Uzbekistan, Iran, Algeria and Italy (FAOSTAT, 2021).

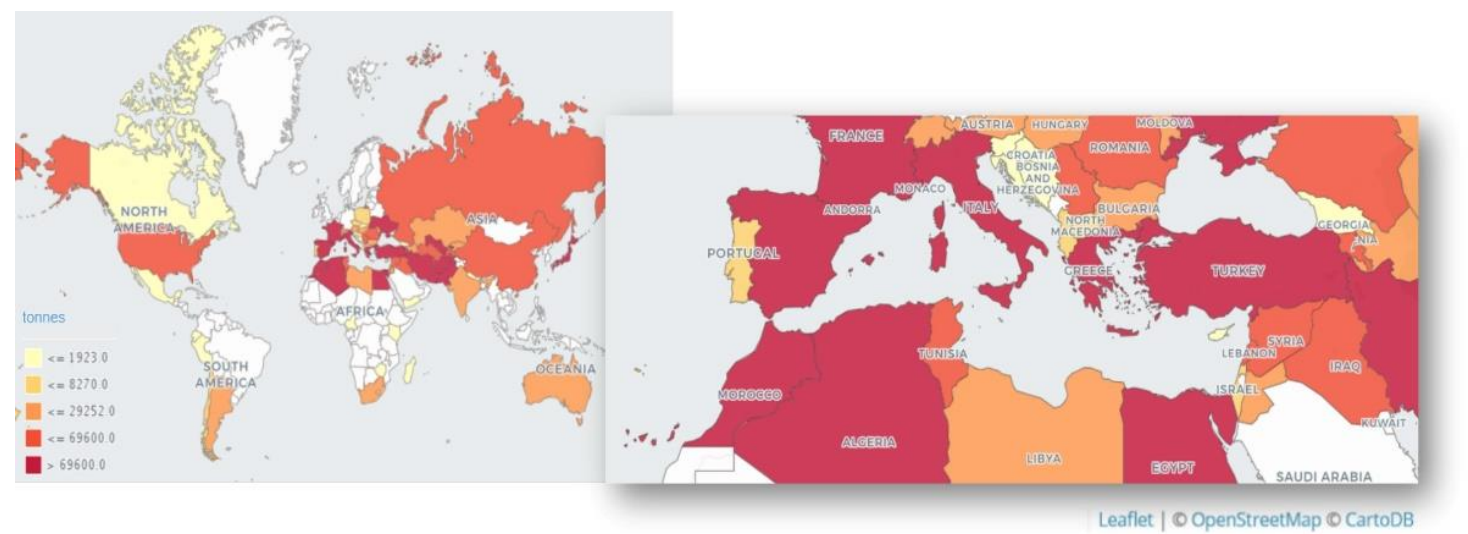

Figure 3. Worldwide and Mediterranean Basin apricot production (t) in 2019 (FAOSTAT, 2021).

Spain was the sixth worldwide producer with 145,836 tonnes of apricot in 2019 (FAOSTAT, 2021). Exports account for $64 \%$ of the Spanish apricot production, which resulted into the main worldwide exporter of fresh apricot, followed by Surinam and Turkey, which is also the main exporter of dried apricots (FAOSTAT, 2021). Spanish apricot exportation is based on early varieties, with a ripening period between April and middle June. Cultivated apricot surface in Spain was 20,240 ha in 2019, being the Region of Murcia and Valencia, the main producers (MAPA, 2020).

\subsection{Apricot cultivation}

\subsubsection{Soil preferences and rootstocks}

Apricot trees are well-adapted to temperate regions of both hemispheres (35-50N and 35-50 $\mathrm{S}$ ), but particularly in the Mediterranean basin countries (Zhebentyayeva et al., 2012). Apricots can support even $-50 \circ \mathrm{C}$, although its buds and flowers could be damaged from -2 to -4 으 (Agustí-Fonfría, 2010; Güneș, 2006). Apricot species prefer deep soils, avoiding clayey and wet or very sandy and dry ones. However, its water requirements are not very elevated. Apricot trees are $\mathrm{K}$ and $\mathrm{N}$ demanding. In fact, $\mathrm{N}$ deficiencies can produce a wrong vegetative development, or even problems during fruit setting (Agustí-Fonfría, 2010).

There is a relatively small amount of research for apricot specific rootstocks as reviewed by Zhebetyayeva et al. (2012). Traditionally, apricot seedlings have been used as rootstocks, like 'Canino 9-7', a 'Canino' clone selected due to the higher germination and better vegetative growth (Orero et 
al., 2004). However, in order to deal with the conditions of soils in the Spanish Mediterranean area and also control the high vigour induced by seedling rootstock, plum based rootstocks were also successfully used (Reig et al., 2018). From them, the hexaploids 'Pollizo de Murcia' (Prunus insititia) and Prunus domestica, the diploid 'Myrobalan' (Prunus cerasifera), and different plum based hybrids as 'Marianna' (Prunus cerasifera $\times$ Prunus munsoniana) became popular. The rootstock-cultivar affinity should be taken into consideration to select the right rootstock as well as climate and soil conditions, like its tolerance to nematode and fungal pathogen and its adaptability to calcareous and harder soil textures (Hernández et al., 2010; Reig et al., 2018).

\subsubsection{Self-compatibility}

On the other hand, apricot exhibits a gametophytic self-incompatibility (GSI) system that prevents selffertilization favoring outcrossing, even though many European apricot varieties are self-compatible (SC) (Muñoz-Sanz et al., 2017b). This trait is very important for orchard production as it could have a significant impact on fruit set and yield as revised by Muñoz-Sanz et al. (2020b). GSI is genetically controlled by a single multiallelic locus, termed S-locus, that contains at least 2 linked genes, one expressed in the pistil (S-RNAse) and the other in the pollen (SFB in Prunus). In apricot, some selfcompatible mutants have been described having a knock-out mutation of SFB, named SFBc (Vilanova et al., 2005). Moreover, another pollen gene, named ParMDO, has been identified at our IVIA's group as necessary for the GSI system in apricot (Muñoz-Sanz et al., 2017a). Nowadays, molecular assisted selection for SC is applied as a routine at the IVIA's apricot breeding program (Muñoz-Sanz et al., 2020a).

\subsubsection{Pests and diseases}

Main apricot pests and diseases are common to other stone fruits as plums, peaches, or cherries. Apricot trees are susceptible to bacterial canker and blast (Pseudeomonas syringae), but also to fungal affections as powdery mildew (Sphaerotheca pannosa and Podosphaera tridactyla), shothole (Wilsonomyces carpophilus) or blossom blight and fruit brown rot (Monilinia laxa) (Agustí-Fonfría, 2010; Ledbetter, 2008).

However, Plum pox virus (PPV) is the most important viral disease affecting stone fruits trees and is a limiting factor for apricot production worldwide (García and Cambra, 2007). PPV is a potyvirus transmitted by aphids in a non-persistent way, being the chemical treatments not effective in preventing plant infection. As observed in Figure 4, PPV symptoms appear on leaves, shoots, flowers, but the most recognizable symptom is the appearance of circular and chlorotic spots or yellow rings in leaves and fruits, which can also affect the stone (García et al., 2014). As a result, fruits become deformed and develop irregular shapes, developing necrotic areas under spots, and becoming noncommercial (García et al., 2014). 

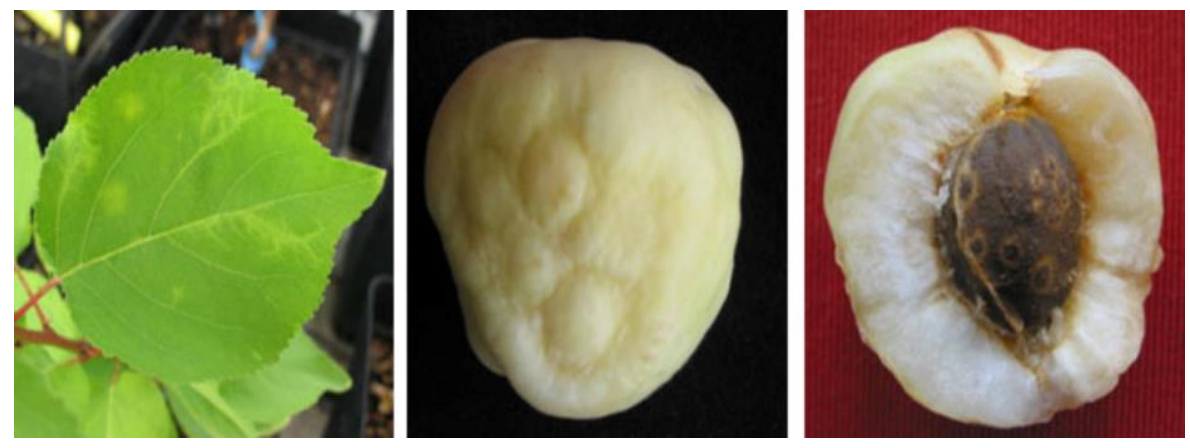

Figure 4. Apricot PPV symptoms in leaves, fruits, and stone (Rubio et al., 2009).

Control measures are based on the use of certified and healthy plants and the eradication of infected trees. However, the time between inoculation and the detection of symptoms together with the persistence of virus reservoirs, make eradication inefficient (Martínez-Gómez et al., 2000). In this scenario, the growth of PPV resistant Prunus cultivars has been pointed out as the ideal long-term solution. After an important germplasm screening, just a handful of North American apricot cultivars were described as resistant and have been used as donors in all apricot breeding programs in progress (Martínez-Gómez et al., 2000). Interestingly, after an important international effort, our group has identified two ParPMC genes, members of a cluster of genes, as host susceptibility paralogous genes required for PPV infection (Zuriaga et al., 2018). Moreover, the presence of a $5 \mathrm{nt}$ deletion within the second intron of ParPMC2 is used as a molecular marker (ParPMC2-del) for assisted selection in our breeding program (Polo-Oltra et al., 2020), avoiding the need for resistance phenotyping, a real bottleneck for any apricot breeding program.

\subsubsection{Fruit quality}

Fruit ripening is a genetic and irreversible process from the physiological point of view (Fujisawa et al., 2013). Apricot is a climacteric fruit (Biale, 1960), hence, during the ripening process the ethylene concentration increases the respiration ratio (Brady, 1987). During fruit development, its main attributes as fruit firmness, soluble solids content, acids, and colour change. In fact, in stone fruits and other Rosaceae species, the highest value of soluble sugar content is reached the end of the ripening process, but contrary, organic acids decrease during fruit ripening due to they are used as respiratory substrate (Chen et al., 2009; Desnoues et al., 2014; Moing et al., 2001; Zhang et al., 2019). Those changes are crucial for the apricot postharvest behaviour and commercialization (Xi et al., 2016). For this reason, there is an interest in the study of the ripening process and its role in fruit quality.

Diversity in pomological parameters, pigments, soluble solids, organic acids, ethylene production, texture and in its maturity date have important effects on fruit quality. Moreover, the pomological and nutraceutical properties depend on apricot varieties, but also on cultural practices, stage of 
development and environmental conditions (Bae et al., 2014; Drogoudi et al., 2008; Ruiz et al., 2005). In this sense, knowing the germplasm available would allow to develop new cultivars that meet the high requirements demanded by consumers but also to study the genetic control of these traits. In a highly competitive scenario, sensorial fruit properties and consumer approval are influenced by volatile compound contents, skin and flesh colour, size, or texture (Naryal et al., 2019; Ruiz and Egea, 2008). In this sense, some studies suggested that consumers are interested in large fruit size, orange colour skin and an intense blush (Piagnani and Bassi, 2013).

On the other hand, an adequate balance among sugars and organic acids is crucial for the consumer due to its influence on taste and flavour (Gurrieri et al., 2001; Kader, 2008; Klee, 2010). Actually, total soluble solid content influences notably the fruit taste (Caliskan et al., 2012), but also some studies in nectarine and peach revealed that the sugar and organic acids ratio and the citric and shikimic acids have a relevant impact on sweetness perception. In addition, other studies also suggested that aroma and taste is influenced by organic acids and sucrose (Colaric et al., 2005).

Sugar composition depends mainly on genetics, but cultivation and environmental factors can affect the total sugar concentration, which increases until reaches a maximum at the ripening (Xi et al., 2016). Moreover, in some apricot cultivars have been found a positive correlation among sugars and anthocyanins in red-blushed cultivars (Huang et al., 2019). The major sugars in apricot are sucrose, followed by glucose, fructose and sorbitol (Akin et al., 2008; Drogoudi et al., 2008; Karabulut et al., 2018; Schmitzer et al., 2011), in accordance with other fruits as peach, litchi and mandarin, in which also the sucrose is the predominant sugar (Xi et al., 2016). However, in plum and apricot sucrose only is detected when fruits reach the full maturation stage (Bae et al., 2014). Regarding organic acids, malic and citric acids are the predominant in most fruits, but the final organic acid concentration is influenced by the balance of organic acid biosynthesis, its metabolism and its vacuolar storage. In apricot, some authors found malic acid as the predominant organic acid (Akin et al., 2008).

As a diet rich in fruits and vegetables can have a positive impact on health or reduce the risk of cardiovascular diseases or hypertension (Hu et al., 2000; Southon and Faulks, 2002), consumer increased its demand in fruits with a high content in phytochemicals with antioxidant potential (Caliskan et al., 2012). Carotenoids, dietary fibbers, phenolic compounds, and vitamins are the predominant bioconstituents with health benefits (Slavin and Lloyd, 2012). In this sense, apricot fruit could be considered a functional food as it is a source of fibre, organic acids, minerals, sugars, anthocyanins, vitamins, provitamin A and ascorbic acid (Akin et al., 2008; Bolin and Stafford, 1974; Dragovic-Uzelac et al., 2007; Hegedüs et al., 2011; Leccese et al., 2008; Moustafa and Cross, 2019; Owais, 2010; Ruiz et al., 2005).

Apricots also have a beneficial effect on health due its content in antioxidant compounds (ErdoganOrhan and Kartal, 2011; Xi and Lei, 2020). Apricots contain phenolic compounds, such catechin, 
epicatechin, p-coumaric acid among others, that exhibit a good antioxidant activity (Arts et al., 2000). However, many factors could influence the fruit antioxidant capacity such as the genotype, geographic region, harvest year or fruit development period (Dragovic-Uzelac et al., 2007; Drogoudi et al., 2008; Hegedüs et al., 2010). The most abundant phenolic compounds in plants are flavonoids, they have an important role in the plant defence system, but also they are reported as relevant for human diet (Giada, 2013). The major phenolic compound in apricots are chlorogenic and neochlorogenic acids, (+)catechin, (-)-epicatechin, and rutin (Radi et al., 1997), which also was found as the predominant phenolic compound in some apricot varieties (Schmitzer et al., 2011). Moreover, phenolic content had a more significant contribution to the total antioxidant capacity in apricots than total carotenoid content (Drogoudi et al., 2008).

Finally, phytocompounds are not equally distributed in fruits. For instance, higher concentrations of organic acids or phenolics have been found in peel than in pulp (Nunes et al., 2008; Schmitzer et al., 2011; Xi et al., 2016) and a similar fruit distribution has been found for the content of aroma volatiles, making the peel of fruits an excellent tissue to explore flavour quality formation (Kader, 2008; Xi et al., 2016).

\subsection{Apricot breeding}

In general, fruit tree breeding programs consisted in biparental crosses between species or varieties with the traits of interest with the aim of obtaining a diverse offspring for them. Once the offspring has been obtained, the phenotypes of interest are selected and tested in different environments. Notably, the development of a new cultivar takes years from pollination and fruit trees breeders need to anticipate cultivar needs at least 10 years into the future, including aspects of production, fruit consumption, climate change or market requirements (Byrne, 2012). Moreover, fruit tree breeding programs have also to deal with the long juvenility periods of these species that affect the breeding cycle. For instance, the length of this period is 3-5 years in Prunus spp. (van Nocker and Gardiner, 2014). Another challenge refers to the high space requirements due to large plant sizes or their high levels of heterozygosity. As an advantage, the vegetative propagation allows to preserve the improved traits easily as it fixes the favourable combinations of the traits.

The developments in biotechnology and genomics have opened the opportunity to address these problems more easily. It is worth highlighting the molecular tools developed in the case of Rosaceae within the framework of international projects RosBREED (www.rosbreed.org/) and FruitBreedomics (www.fruitbreedomics.com/) or Genome Database for Rosaceae (GDR, https://www.rosaceae.org). Biotechnological tools such as genetic transformation could accelerate breeding programs. However, the transformation efficiency depends on the species, being too low in the case of apricot (Petri et al., 
2008). The hexaploid plum is the model species for Prunus spp genetic transformation (Petri et al., 2012). Anyway, the key aspect is still knowing the genes involved in the control of the trait in order to use them as a target in transformation or editing assays and this still requires a lot of basic research. For this purpose, nowadays, as the cost of DNA sequencing is more and more cheap, the phenotyping process is the actual bottleneck of any breeding program.

\subsubsection{Main programs in the world}

Due to changes of industry and consumers requirements, the development of new apricot cultivars is continuous. In Europe, The Community Plant Variety Office (CPVO) have a list of 322 apricot cultivars, which includes 148 under active protection of plant variety rights and other 31 under analysis (https://cpvo.europa.eu/en). Despite these numbers, the number of apricot specific breeding programs is lower than in other fruit trees species such as peach or apple (Zhebentyayeva et al., 2012). The main objectives in European apricot breeding programs are resistance to biotic stresses, environmental adaptability, the extension of the harvest season, quality for fresh and processed fruits and pomological traits (Bassi and Audergon, 2006). As it is a species with very low plasticity, each region develops its own varieties. However, the available PPV resistant cultivars (Martínez-Gómez et al., 2000), used as donors in all the apricot breeding programs, are not well adapted to the Southern European conditions, hampering the process to develop new cultivars in these regions.

The main breeding achievements around the world were reviewed by Zhebentyayeva et al. (2012). The oldest ongoing apricot breeding program started in 1925 at the Nikita Botanical Gardens in Yalta, Crimea, Ukraine. As far as we know, not too much information is publicly available on the apricot breeding programs of the main producing countries, such as Turkey or Iran.

In Italy, the 5th apricot producer, 4 public breeding programs are in progress at the Universities of Milano, Bologna and Pisa and the Unità di Ricerca per la Frutticoltura di Caserta (Bassi, 1995; Zhebentyayeva et al., 2012;).

In Greece, the apricot breeding program started in 1989 in order to deal with the sharka disease. North-American PPV-resistant cultivars were crossed with the local cultivar named 'Bebecou' to look for resistance to the aggressive strain PPV-M (Karayiannis et al., 2006a and 2006b).

In France, the INRA and the Sica Centrex in collaboration with the Cep Innovation, are carrying out an apricot breeding program focused on the obtention of new varieties with interesting agronomical and gustative traits, particularly a largest maturity time, regular production, excellent firmness, and postharvest behaviour. In addition, the program is also aimed to obtain new Sharka resistant accessions with an unambiguous varietal segmentation in terms of skin and flesh colouration (Audergon et al., 2009 and 2011).

Outside of the European Community, apricot breeding programs are in progress on all continents, 
excepts Antarctica. In the regions where PPV has been of lesser importance, the apricot breeding programs were focused on other objectives. In Australia, the South Australian Research \& Development Institute (SARDI) started 35 years ago a national breeding program in order to develop apricot cultivars for the fresh market and dried industries (Graetz, 2018). Their goals were related to a better eating and fruit quality, acceptable post-harvest properties, and a good performance with mechanised production systems, but also maintaining traditional characteristics as full colour (Graetz, 2018).

In North America, California is the major region of apricot production with around the $86 \%$ of harvested production, however, in some regions production was limited. Early apricot breeding programs started in 1950's, and goals were focused mainly on productive varieties identification (Ledbetter, 2010). Apricot breeding at the Agricultural Research Service laboratory in Fresno, CA began in 1955. Early efforts in apricot were focused to identify productive selections, but also on the high fruit acidity. In addition, large, firm, and coloured apricot selections were obtained. Moreover, breeding efforts also are focused to obtain fresh apricots with a high quality in terms of sweetness (Ledbetter, 2010). Regarding pests and diseases, despite that PPV has been maintained under control and its impact is minimal in this country, the development of new varieties well-adapted to California conditions and PPV-resistant should be a high priority future objective in order to deal with futures PPV breakouts (Ledbetter, 2010).

In Asia, Chinese breeding programs efforts had, as a result, an expansion of the apricot ripening season, an increase of fruit and kernel size and also production. In addition, breeding programs also had as a result an improved firmness, flavour, and aroma characteristics (Badenes and Hutagalung, 2012). In Japan, breeders are focused on fruit processing abilities, but also on fruit colour and pigmentation, a later flowering and early fruit ripening.

\subsubsection{Breeding programs in Spain}

Two public institutions are carrying out apricot breeding programs in Spain, the Centro de Edafología y Biología Aplicada del Segura (CEBAS-CSIC) and the IVIA, and both started with the main purpose of developing PPV-resistant cultivars. For this purpose, as explained previously, the available PPV-donors that could be used were not well-adapted to the Spanish productive areas. At CEBAS-CSIC, the apricot breeding program started in 1991 and as a result, some PPV-resistant cultivars as Rojo Pasión,' 'Selene' or 'Murciana', but also with high fruit quality and late-ripening as 'Dorada' have been developed (Egea et al., 2004a, 2004b, 2005).

The apricot breeding program at IVIA started in 1993 in order to obtain new apricot varieties with high fruit quality, resistant to PPV and well-adapted to the Southern European environment (MartínezCalvo et al., 2009). The mid-early ripening cultivars "Dama Taronja" and "Dama Rosa" were recently 
released (Badenes et al., 2018) (Figure 5). In order to increase the efficiency of the breeding program, a marker-assisted selection (MAS) strategy is routinely implemented to identify PPV resistant and selfcompatible seedlings (Muñoz-Sanz et al., 2020a; Polo-Oltra et al., 2020).

A

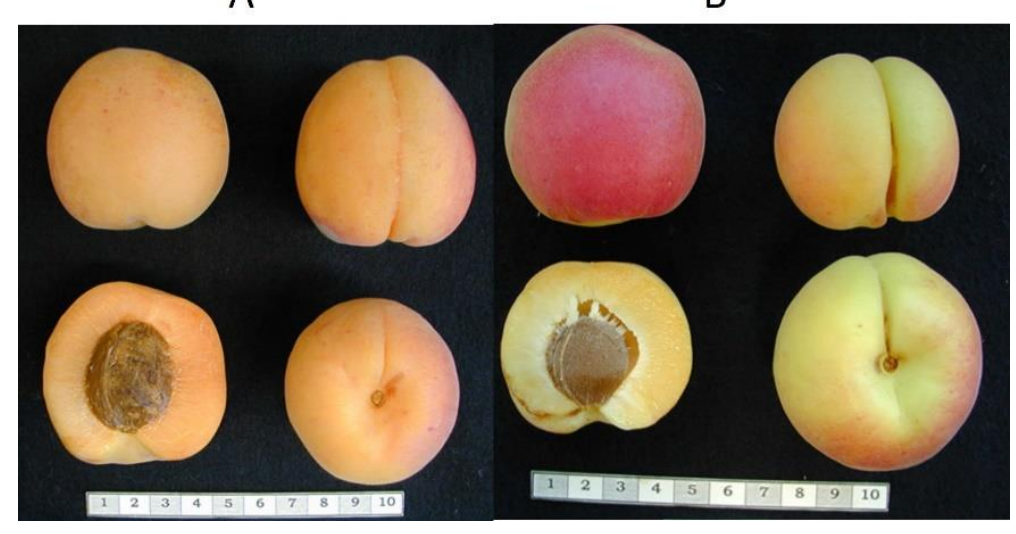

Figure 5. PPV-resistant 'Dama Taronja' (A) and 'Dama Rosa' (B) apricot fruits (Badenes et al., 2018). Scale bars in centimetres.

\subsubsection{Germplasm and genetic resources for apricot breeding}

The first step of any breeding program is the identification (or creation) of genetic variation that could be used as a source of an interesting trait. For this purpose, the genebanks are a key tool as they locate, collect and conserve plants considered of priority interest for our society or scientific knowledge aimed at optimizing the conservation and use of plant genetic resources, for example, in breeding programs (Tanksley and McCouch, 1997). Interestingly, the biggest seeds germplasm bank is the Svalbard Global Seed Vault (SGSV) (https://seedvault.nordgen.org), located in Norway, which keeps seed samples duplicates of the crop diversity stored in the world's genebanks following the FAO's genebank standards.

Regarding fruit tree species, The National Clonal Germplasm Repository in Davis, CA (USA) is a genebank of the United States Department of Agriculture (USDA) and the Agricultural Research Service (ARS). The collection maintains crops adapted to Mediterranean climates and its Prunus spp. collection includes more than 90 taxa and 1600 accessions (Preece and Aradhya, 2013). This collection is of great interest as an important source of genes for fruit tree breeding. Moreover, as explained before, due to the low plasticity of the apricot species, each institution usually maintains a small collection of local adapted accessions that are useful for their breeding programs. In this sense, Badenes and Zuriaga (2016) reviewed the use of these local accessions and the importance of maintaining them in germplasm collections in order to avoid their loss. 


\subsubsection{Genetic control of fruit quality traits}

Regarding fruit quality, previous studies in Prunus revealed that the phenotypic expression of most fruit quality traits is quantitative and depends on complex biochemical processes and metabolic pathways, having a multigenic control (Illa et al., 2011). This, makes difficult to identify linked markers to apply MAS (García-Gómez et al., 2018). With this purpose, QTLs identification of traits related to soluble solids, acid content, skin or flesh colour has been studied (García-Gómez et al., 2019).

A genetic map in Prunus spp. described QTLs in LG1 related with fruit mass and citric acid (Ogundiwin et al., 2009). Regarding soluble solid content and sugar metabolism, in Prunus spp some QTLs related with main sugars content have been found in LG1, 4, 5, 6, and 7 (Dirlewanger et al., 2006; Etienne et al., 2002; García-Gómez et al., 2019; Ogundiwin et al., 2009; Quilot et al., 2004). In peach, LG5 also has been related with some traits associated with skin colour, with two candidate genes coding enzymes involved in flavonoids metabolic pathway (Quilot et al., 2004).

Concerning genetic control of phenolic compounds, some QTLs have been described in apricot (GarcíaGómez et al., 2019; Salazar et al., 2017). Various QTLs related with skin and flesh colour have been located in LG3 and a gene encoding for a MYB transcription factor has also been described in this region (García-Gómez et al., 2019; Salazar et al., 2017). Regarding the genetic control of sugars metabolism, a sucrose-synthase (SUS1), a sucrose-phosphate-synthase (SPS2) and a neutral invertase (Ivr), have been described as regulation steps in sugar biosynthesis (Zhang et al., 2019). Moreover, in LG4 some candidate genes involved in diglucose, and D-mannose binding have been described for apricot (García-Gómez et al., 2019), in agreement with other authors who found in LG4 the most relevant QTLs related to ripening time, fruit development and soluble solid content (Ruiz et al., 2010; Salazar et al., 2013, 2016; Socquet-Juglard et al., 2013).

However, the described QTLs related to quality-traits in stone fruits, and particularly in apricot, is limited (Abbott et al., 2008), being most of them are reported in peach. Moreover, none of the putative QTLs controlling sugar-related traits described in peach have been fine-mapped (Cirilli et al., 2016). This evidences the need to extend the knowledge of genetic control and characterization of quality traits, particularly of the metabolic pathway of sugars or phenolic compounds due to the demand of consumers. 
OBJECTIVES 
Consumers are increasingly demanding high-quality products. In a globalized scenario, the development of new varieties that meet these requirements is essential for the apricot crop to remain competitive. In this sense, the overall objective of this thesis is the germplasm screening for the identification of sources of nutraceutical compounds that could be used as donors at the IVIA's apricot breeding program. For this purpose, this thesis has pursued the following 3 specific objectives:

1. Screening of the content of main sugars, organic acids, phenolic compounds, and ascorbic acid in a set of accessions of the IVIA's apricot germplasm collection.

2. Characterization of the expression of the main genes involved in the phenolic and sugar metabolic pathways and its relationship with fruit content.

3. Study the genetic contribution of the cultivar 'Goldrich', used traditionally as the main donor of PPV resistance, to its offspring regarding phenolic compounds content. 


\section{CHAPTER I: Nutraceutical profiles of apricots (Prunus armeniaca L.) as a source of fruit quality traits for breeding.}

This section is based on an article accepted for publication in the Spanish Journal of Agricultural Research (in press) (Document S1).

Authors contributions:

- $\quad$ Helena Gómez-Martínez: Data curation, writing original draft, formal analysis.

- A. Bermejo: Data curation, analysis of compounds.

- M.L. Badenes: Project administration, writing original draft.

- $\quad$ E. Zuriaga: Supervision, writing original draft. 


\section{I.1. Abstract}

In a social context of increasingly concern about healthy diets, the development of new varieties with enhanced content in nutraceutical compounds is an increasingly important objective of the fruit breeding programs currently developed. In this sense, apricot is a fruit crop very appreciated by consumers due to its organoleptic characteristics, but also plays an important role in human nutrition due to its content of phytocompounds as sugars, organic acids, vitamins, and polyphenols. In this work, new selections from the apricot breeding program carried out at the Instituto Valenciano de Investigaciones Agrarias (IVIA) and traditional varieties have been analysed aimed at identifying sources of genetic variation for fruit quality. For this purpose, sugar content, organic acids and ascorbic acid were studied during 3 crop years. Results revealed sucrose and glucose as the major sugars, malic and citric acid as the main organic acids, and diverse ascorbic acid content among the cultivars studied. Results obtained pointed some accessions as potential sources to increase fruit quality. In addition, the study showed that apricot peel is an excellent source of nutraceutical compounds. Moreover, this study opens up new possibilities for future work to study the genetic control of these traits in apricot.

\section{Keywords:}

sugars, ascorbic acids, organic acids, breeding

\section{Abbreviations used:}

DW: Dry Weight

FW: Fresh Weight

PPV: Plum Pox Virus

SI: Sweetness index

TSI: Total Sweetness index 


\subsection{Introduction}

The increasing demand for safe, healthy, and nutritious food by consumers, turn the internal quality of the fruit into one of the main goals of the food industry. In this sense, plants and some fruits become a useful source of compounds with a relevant role in improving health (Slavin and Lloyd, 2012; Vieira da Silva et al., 2016). In fact, plant extracts and their bioactive compounds are used by the industry to produce functional food (Azmir et al., 2013). For this reason, those fruits with high content of these compounds are of high interest for the industry. In this sense, nutraceutical profiles can be used for promotion of fruit consumption as a natural functional food.

Apricot (Prunus armeniaca L.) is a stone fruit crop species with a large tradition in the Mediterranean basin countries. World apricot production reached 4.08 million tonnes in 2019, being Turkey, Uzbekistan, Iran, Italy, Algeria, and Spain as main producers (FAOSTAT, 2021). Despite its wide geographical spread, each region usually grows locally adapted apricot cultivars because this species has very specific ecological requirements. In this sense, significant breeding efforts have been undertaken (Zhebentyayeva et al., 2012), leading to a rich diversity apricot germplasm in terms of fruit morphology, harvest season or biotic and abiotic stresses. Apricots are consumed in multiple and diverse ways, including fresh or processed fruits (as dried, canned, jam, juice or even liquors), and the apricot kernel oil is also used for medicinal purposes (Zhebentyayeva et al., 2012). Apricots are an important source of sugars, fiber, proteins, minerals, and vitamins (Moustafa and Cross, 2019; Sochor et al., 2010). However, pomological and nutraceutical properties depend on varieties, cultivation systems, fruit storage conditions or developmental stages (Ruiz et al., 2005).

In terms of fruit consumption, organoleptic characteristics are one of the main factors for consumers decision. Notwithstanding, nutraceutical compounds interact with each other and influence the quality properties making it difficult to handle. For instance, the flavour is provided by sucrose, malic acid, and volatiles (Xi et al., 2016), being sugar and organic acid balance relevant for sweetness. From them, fructose and sucrose are the prominent contributors to sweetness, being the most important sensory quality for consumer satisfaction (Fan et al., 2017). Similar results have been found in peach, whose sweetness depends on the overall sugar amount as well as in the specific relative amount of each individual sugar (Kroger et al., 2006). Regarding the apricot nutraceutical profile, previous studies have also found glucose and sucrose as the major sugars in both flesh and peel (Xi et al., 2016). Moreover, during the fruit ripening a high number of molecular and metabolic changes occur that have a relevant effect in fruit properties (D'Ambrosio et al., 2013; Karlova et al., 2014; Osorio et al., 2013; Seymour et al., 2013). In this sense, García-Gómez et al. (2021) reviewed current knowledge of the molecular bases of fruit ripening process in Prunus species due to its importance for breeding. For instance, organic acids increase during the early stages of fruit development and decrease when fruits were full-ripped, 
being malic the most important organic acid in apricot (Xi et al., 2016). Additionally, fruits and vegetables constitute the main source of ascorbate in the human diet, so rising its content in highly consumed fruits would clearly have an impact on human nutrition (Fenech et al., 2019). Moreover, ascorbate content has been also related with elevated stress tolerance (Fenech et al., 2019). In fact, foliar application of ascorbic acid on peach trees resulted in improving the yield and fruit quality (Sajid et al., 2017). Previous studies found that vitamin C content in apricot could reach up to $100 \mathrm{mg} / 100 \mathrm{~g}$ dry weight (DW) (Akin et al., 2008), showing the potential of this species as a source of this vitamin. In conclusion, apricot germplasm represents a diverse source of phytocompounds that can be exploited for breeding purposes in order to develop new varieties with higher content of these nutraceutical compounds. The apricot breeding program at the Instituto Valenciano de Investigaciones Agrarias (IVIA) has the purpose of obtaining new varieties, with high fruit quality, resistant to the Plum Pox virus (PPV), self-compatibles and well-adapted to the Southern European environment (Martínez-Calvo et al., 2009). PPV is the main limiting factor for apricot production worldwide, hence, during the last decades, development of PPV resistant varieties has been the main objective of almost any apricot breeding program (Polo-Oltra et al., 2020). However, for this purpose, just some North American cultivars not well-adapted to Mediterranean conditions were identified and used as resistance donors (Martínez-Gómez et al., 2000). This represents a challenge especially in the current climate change scenario affecting the Mediterranean basin, with increasingly mild winters.

The objective of the present work is to assess the fruit quality characterization of 1 North-American, 3 Spanish (Valencian Community) and 9 accessions from the IVIA's apricot breeding program aimed at identifying the most convenient genotypes for increasing the fruit quality of apricot while keeping the adaptability to warm winters. In this study we analyse sugars (sucrose, fructose, and glucose), ascorbic acid, and organic acids (citric, malic, succinic and fumaric) during 3 cropping seasons.

\section{I.3. Material and Methods}

\section{I.3.1. Plant Material}

Thirteen apricot genotypes were used, including 3 well-known cultivars from the Mediterranean Basin ('Canino', 'Mitger' and 'Tadeo'), 1 North-American ('Goldrich'), and 9 selections from the IVIA's breeding program resistant to PPV ('Dama Rosa', 'Dama Taronja', 'GG9310', 'GG979', 'GP9817', 'HG9821', 'HG9850', 'HM964' and 'SEOP934'). Pedigree information could be checked at Polo-Oltra et al., (2020). All of them are kept at the collection of the IVIA in Moncada (Valencia, Spain). Five fruits per tree were harvested at the ripening stage during 3 growing seasons (2016, 2017 and 2019) and used for pomological and nutraceutical analyses. For each fruit, the peel was separated from the flesh 
with a peeler. A mix of 5 fruits (peel or flesh, respectively) was frozen with liquid nitrogen and kept at -80 C $\mathrm{C}$ until processing. Peel samples were freeze-dried and powdered. Tissue homogenization was carried out using a Polytrom 3100 (Kinematica AG, Switzerland) and a vortex for the flesh and peel samples, respectively.

\section{I.3.2. Sample processing and HPLC analysis}

For sample processing, $1 \mathrm{~g}$ of flesh or $10-20 \mathrm{mg}$ of freeze-dried peel were mixed with $1.5 \mathrm{~mL}$ of $5 \%$ metaphosphoric acid solution, $1 \mathrm{~mL}$ of water of LC-MS grade and $1 \mathrm{~mL}$ of $0.1 \% \mathrm{H}_{2} \mathrm{SO}_{4}$ solution for ascorbic acid, sugars, and organic acids extraction, respectively. Then the sample was homogenized and centrifuged at 4 으 for $20 \mathrm{~min}$ at $8.050 \mathrm{xg}$.

Compounds were identified on the bases of comparing their retention times, UV-vis spectra and mass spectrum data with authentic standards obtained from Sigma-Aldrich using an external calibration curve. In addition, standards were run daily with samples for validation. All the solvents used were of LC-MS grade. Three samples per cultivar were analysed and all the samples were run in triplicate. The Empower 2 software (Waters, Spain) was used for data processing.

\section{I.3.2.1. Ascorbic acid}

Total ascorbic acid was extracted according to the method previously described by Cano and Bermejo, (2011) adapted to a microliter format (Sdiri et al., 2012) and using DL-dithiothreitol (DTT) as reducing reagent of dehydroascorbic acid to ascorbic acid. After centrifugation, $1 \mathrm{~mL}$ of supernatant was mixed with $200 \mu \mathrm{L}$ of DTT ( $20 \mathrm{mg} / \mathrm{mL}$ ) and maintained for $2 \mathrm{~h}$ in the dark, then filtered through $0.45 \mu \mathrm{m}$ filter. It was analysed by HPLC-DAD in an Alliance liquid chromatographic system (Waters, Barcelona, Spain) equipped with a 2695 separation module coupled to a 2996 photodiode array detector, and a reversephase $\mathrm{C}_{18}$ column Tracer Excel $5 \mu \mathrm{m} 120$ OSDB (250 mm x $4.6 \mathrm{~mm}$ ) (Teknokroma, Barcelona, Spain) with an isocratic mobile phase of methanol:0.6\% acetic acid (5:95) at a flow rate of $1 \mathrm{~mL} / \mathrm{min}$, and injection volume was $5 \mu \mathrm{L}$. The quantification was performed at $245 \mathrm{~nm}$.

\subsubsection{Sugars}

Sucrose, glucose, and fructose were extracted as described by Sdiri et al., (2012). After centrifugation, samples were filtered through a $0.45 \mu \mathrm{m}$ nylon filter and analysed by an HPLC system equipped with a Waters 515 HPLC pump, a Waters 2414 refractive index detector, a 5- $\mu \mathrm{m}$ Tracer Carbohydr column (250 mm x $4.5 \mathrm{~mm}$ ) (Teknokroma, Barcelona, Spain), and a 20- $\mu \mathrm{L}$ loop Rheodyne injector were used for the sugar analysis. The mobile phase was composed of acetonitrile and water (75:25) at a flow rate of $1.0 \mathrm{~mL} / \mathrm{min}$. 


\section{I.3.2.3. Organic acids}

Citric, malic, succinic and fumaric acids were extracted as described by Sdiri et al., (2012). After centrifugation, the supernatant was filtered through a $0.45 \mu \mathrm{m}$ filter, analysed by HPLC-DAD and confirmed by HPLC-MS under electrospray ion negative conditions using a ZQ2000 mass detector. The sample temperature was $5{ }^{\circ} \mathrm{C}$ and column temperature was $35^{\circ} \mathrm{C}$. Capillary voltage was $3.0 \mathrm{kV}$, cone voltage was $23 \mathrm{~V}$, source temperature was $100^{\circ} \mathrm{C}$, desolvation temperature was $200^{\circ} \mathrm{C}$ and desolvation gas flow was $400 \mathrm{~L} / \mathrm{h}$. Full data acquisition was performed by scanning from 100 to 400 uma in the centroid mode. An ICSep ICE-COREGEL 87H3 column (Transgenomic, UK), an ICSep ICE-COREGEL 87H guard kit, and an automatic injector were used for chromatographic separation. The solvent system was an isocratic mobile phase of $0.1 \% \mathrm{H}_{2} \mathrm{SO}_{4}$ solution. The total run time was $20 \mathrm{~min}$ at $0.6 \mathrm{~mL} / \mathrm{min}$, and the injection volume was $5 \mu \mathrm{L}$.

\section{I.3.3. Pomological characterization}

Ten fruit variables were studied according to the apricot descriptor guidelines published by the International Union for the Protection of the Obtained Vegetables (CPVO and EU, 2008). A digital calibrator Mahr 16 EX was used for length measures. Fruit weight was measured in a precision scale COBOS ( $\max 12 \mathrm{~kg}, \mathrm{~d}=1 \mathrm{~g}$ ). Firmness was measured using an EZ-L Test (Shimadzu, Kyoto, Japan) with an $8 \mathrm{~mm}$ cylindrical plunger. Colour related traits were determined by visual inspection and codified as qualitative traits following the CPVO descriptor.

\section{I.3.4. Data analysis}

Data were analysed with R (R Core Team, 2012) using R-studio software (v.3.5.3) with stats, ggbiplot, readxl, graphics and grDevices packages. Normality and homoscedasticity were checked using ShapiroWilk and Bartlett tests, respectively. Next, the non-parametric Kruskal-Wallis test was used to make all samples comparisons. Notched Box-and-whiskers plots were used to determine significant differences between groups. Correlation coefficients among the variables were determined using the Spearman method. Principal component analysis (PCA), using centered and scaled data, was conducted to visualize the relationships between accessions and variables.

\section{I.3.5. Sweetness Index (SI) and Total Sweetness Index (TSI)}

In order to determine the sweetness perception of fruits, both index were calculated according to (Magwaza and Opara, 2015) following the equations:

$$
\begin{aligned}
& \mathrm{SI}=(1.00 \times[\text { glucose }])+(2.30 \times[\text { fructose }])+(1.35 \times[\text { sucrose }]) \\
& \mathrm{TSI}=(1.00 \times[\text { sucrose }])+(0.76 \times[\text { glucose }])+(1.50 \times[\text { fructose }])
\end{aligned}
$$




\section{I.4. Results}

Pomological and metabolic data obtained here were submitted to statistical analysis in order to check the presence of significant differences between genotypes and/or years. In all cases, data showed no normality and homoscedasticity according to the Shapiro-Wilk and Bartlett test, respectively, violating ANOVA assumptions. For this reason, the non-parametric Kruskal-Wallis test was used to check differences between years and/or genotypes in all cases.

\section{I.4.1. Sugars}

Fructose, glucose and sucrose content in peel and flesh showed significant differences $(p \leq 0.05)$ between the accessions analysed (Table 2, Figure S1).

Table 2. Profiles of sugar content in flesh (g/100 g fresh weight (FW)) and peel (g /100 g Dry Weight (DW)) during 2016, 2017 and 2019.

\begin{tabular}{|c|c|c|c|c|c|c|c|c|c|c|c|c|}
\hline \multirow{2}{*}{$\begin{array}{l}\text { FLESH } 2016 \text { FW } \\
\text { Genotype }\end{array}$} & \multicolumn{3}{|c|}{ Fructose } & \multicolumn{3}{|c|}{ Glucose } & \multicolumn{3}{|c|}{ Sucrose } & \multicolumn{3}{|c|}{ Total } \\
\hline & Mean & sd & Significance & Mean & sd & Significance & Mean & sd & Significance & Mean & sd & Significance \\
\hline 'Goldrich' & 0,23 & 0,04 & ef & 1,34 & 0,24 & c & 5,52 & 0,01 & bc & 7,09 & 0,29 & bcd \\
\hline 'Canino' & 0,08 & 0,01 & a & 0,68 & 0,03 & $\mathrm{a}$ & 7,08 & 0,23 & $d$ & 7,85 & 0,27 & de \\
\hline 'Mitger' & 0,38 & 0,04 & g & 1,89 & 0,04 & de & 4,24 & 0,16 & $\mathrm{a}$ & 6,52 & 0,16 & $a b$ \\
\hline 'Tadeo' & 0,48 & 0,02 & $\mathrm{~h}$ & 3,11 & 0,06 & g & 7,16 & 0,55 & $d$ & 10,75 & 0,48 & $\mathrm{~g}$ \\
\hline 'Dama Rosa' & 0,24 & 0,01 & ef & 2,20 & 0,03 & $f$ & 8,62 & 0,72 & e & 11,06 & 0,68 & $\mathrm{~g}$ \\
\hline 'Dama Taronja' & 0,29 & 0,04 & $f$ & 2,08 & 0,08 & ef & 6,89 & 0,18 & d & 9,25 & 0,06 & $f$ \\
\hline 'GG9310' & 0,17 & 0,02 & bcde & 1,02 & 0,06 & $b$ & 6,78 & 0,37 & d & 7,97 & 0,45 & de \\
\hline 'GG979' & 0,10 & 0,01 & $a b$ & 1,29 & 0,00 & c & 4,47 & 0,23 & $\mathrm{a}$ & 5,86 & 0,22 & a \\
\hline 'GP9817' & 0,15 & 0,02 & bcd & 1,02 & 0,06 & $b$ & 6,38 & 0,28 & $\mathrm{~cd}$ & 7,55 & 0,32 & cde \\
\hline 'HG9821' & 0,20 & 0,01 & de & 1,16 & 0,01 & bc & 6,75 & 0,16 & $d$ & 8,11 & 0,15 & $\mathrm{e}$ \\
\hline 'HG9850' & 0,13 & 0,01 & $a b c$ & 0,98 & 0,03 & $b$ & 4,65 & 0,01 & $a b$ & 5,75 & 0,03 & a \\
\hline 'HM964' & 0,20 & 0,01 & cde & 0,94 & 0,01 & $b$ & 5,50 & 0,10 & bc & 6,64 & 0,10 & $a b c$ \\
\hline 'SEOP934' & 0,28 & 0,02 & $f$ & 1,76 & 0,05 & d & 10,30 & 0,03 & $f$ & 12,34 & 0,04 & $\mathrm{~h}$ \\
\hline PEEL 2016 DW & \multicolumn{3}{|c|}{ Fructose } & \multicolumn{3}{|c|}{ Glucose } & \multicolumn{3}{|c|}{ Sucrose } & \multicolumn{3}{|c|}{ Total } \\
\hline Genotype & Mean & sd & Significance & Mean & sd & Significance & Mean & sd & Significance & Mean & sd & Significance \\
\hline 'Goldrich' & 7,03 & 0,94 & d & 18,75 & 2,54 & de & 12,41 & 1,34 & a & 38,19 & 4,67 & a \\
\hline 'Canino' & 2,85 & 0,04 & a & 12,62 & 0,89 & $b$ & 33,13 & 1,84 & $f$ & 48,60 & 2,71 & bcd \\
\hline 'Mitger' & 6,07 & 0,39 & $\mathrm{~cd}$ & 20,94 & 0,76 & ef & 23,20 & 1,45 & bcde & 50,21 & 1,10 & $\mathrm{~cd}$ \\
\hline 'Tadeo' & 4,95 & 0,35 & bc & 23,66 & 0,40 & $f$ & 26,39 & 1,34 & cde & 57,86 & 1,73 & $d$ \\
\hline 'Dama Rosa' & 4,87 & 0,74 & $\mathrm{bc}$ & 19,32 & 3,05 & def & 18,16 & 1,89 & $a b$ & 42,36 & 5,66 & $a b c$ \\
\hline 'Dama Taronja' & 4,61 & 0,18 & $\mathrm{~b}$ & 18,30 & 0,18 & cde & 17,30 & 0,87 & $a b$ & 40,21 & 0,75 & $a b$ \\
\hline 'GG9310' & 4,46 & 0,28 & $b$ & 13,75 & 2,35 & bc & 22,56 & 0,88 & bcd & 40,77 & 1,91 & $a b c$ \\
\hline 'GG979' & 3,84 & 0,68 & $a b$ & 13,06 & 0,90 & $b$ & 21,22 & 1,12 & bc & 40,31 & 2,22 & $a b$ \\
\hline 'GP9817' & 4,29 & 0,11 & $b$ & 13,94 & 0,77 & bc & 26,22 & 2,12 & cde & 44,44 & 2,80 & $a b c$ \\
\hline 'HG9821' & 4,99 & 0,22 & bc & 12,71 & 0,33 & $b$ & 28,59 & 0,72 & def & 46,29 & 0,45 & $a b c$ \\
\hline 'HG9850' & 3,77 & 0,04 & $a b$ & 18,02 & 0,32 & cde & 25,99 & 2,41 & cde & 47,78 & 2,31 & $a b c$ \\
\hline 'HM964' & 3,69 & 0,29 & $a b$ & 7,83 & 0,99 & a & 28,31 & 2,64 & def & 43,41 & 2,25 & $a b c$ \\
\hline 'SEOP934' & 3,89 & 0,11 & $a b$ & 15,54 & 1,26 & bcd & 28,96 & 4,35 & ef & 48,39 & 5,61 & bcd \\
\hline
\end{tabular}


Table 2 (cont.)

\begin{tabular}{|c|c|c|c|c|c|c|c|c|c|c|c|c|}
\hline \multirow{2}{*}{$\begin{array}{l}\text { PEEL } 2017 \text { DW } \\
\text { Genotype }\end{array}$} & \multicolumn{3}{|c|}{ Fructose } & \multicolumn{3}{|c|}{ Glucose } & \multicolumn{3}{|c|}{ Sucrose } & \multicolumn{3}{|c|}{ Total } \\
\hline & Mean & sd & Significance & Mean & sd & Significance & Mean & sd & Significance & Mean & sd & Significance \\
\hline 'Goldrich' & 6,30 & 0,50 & de & 10,01 & 0,98 & $a b$ & 4,63 & 0,33 & $a$ & 23,68 & 1,20 & $a b$ \\
\hline 'Canino' & 4,75 & 0,64 & bc & 15,92 & 1,97 & d & 27,11 & 0,51 & de & 47,78 & 2,76 & $d$ \\
\hline 'Mitger' & 6,02 & 0,35 & cde & 24,48 & 1,53 & e & 31,96 & 2,95 & ef & 62,46 & 1,80 & fg \\
\hline 'Tadeo' & 6,64 & 0,11 & e & 15,75 & 0,49 & $d$ & 23,70 & 0,43 & $d$ & 53,27 & 0,27 & de \\
\hline 'Dama Rosa' & 2,73 & 0,11 & a & 6,24 & 0,57 & a & 7,83 & 0,22 & $a b$ & 16,80 & 0,50 & $a$ \\
\hline $\begin{array}{l}\text { ‘Dama } \\
\text { Taronja' }\end{array}$ & 6,48 & 0,38 & de & 14,41 & 0,18 & bcd & 11,50 & 0,93 & $\mathrm{bc}$ & 32,39 & 1,12 & bc \\
\hline 'GG9310' & 5,72 & 0,04 & bcde & 10,69 & 0,37 & $a b c$ & 16,57 & 0,33 & c & 32,97 & 0,34 & c \\
\hline 'GG979' & 6,54 & 0,67 & de & 9,41 & 0,66 & a & 14,85 & 1,40 & c & 31,89 & 0,21 & $\mathrm{bc}$ \\
\hline 'GP9817' & 6,90 & 0,59 & e & 14,69 & 1,30 & $\mathrm{~cd}$ & 16,09 & 1,15 & c & 37,68 & 2,92 & c \\
\hline 'HG9821' & 6,28 & 0,29 & de & 14,58 & 1,85 & bcd & 46,88 & 4,77 & $\mathrm{~g}$ & 67,74 & 6,76 & g \\
\hline 'HG9850' & 4,85 & 0,72 & bc & 14,28 & 2,25 & bcd & 30,37 & 1,12 & ef & 49,50 & 1,67 & de \\
\hline 'HM964' & 4,58 & 0,42 & b & 16,75 & 2,93 & d & 36,43 & 1,84 & $f$ & 57,76 & 4,80 & ef \\
\hline 'SEOP934' & 5,21 & 0,24 & bcd & 9,62 & 0,12 & a & 16,07 & 2,98 & c & 32,70 & 3,56 & $\mathrm{bc}$ \\
\hline
\end{tabular}

\begin{tabular}{|c|c|c|c|c|c|c|c|c|c|c|c|c|}
\hline \multirow{2}{*}{$\begin{array}{l}\text { PEEL } 2019 \text { DW } \\
\text { Genotype }\end{array}$} & \multicolumn{3}{|c|}{ Fructose } & \multicolumn{3}{|c|}{ Glucose } & \multicolumn{3}{|c|}{ Sucrose } & \multicolumn{3}{|c|}{ Total } \\
\hline & Mean & sd & Significance & Mean & sd & Significance & Mean & sd & Significance & Mean & sd & Significance \\
\hline 'Goldrich' & 6,63 & 0,74 & $f$ & 18,93 & 1,55 & c & 17,80 & 0,75 & a & 43,37 & 2,86 & $a b c$ \\
\hline 'Canino' & 2,26 & 0,28 & a & 14,65 & 0,46 & $a b c$ & 45,03 & 1,44 & e & 61,93 & 1,84 & de \\
\hline 'Mitger' & 6,03 & 0,33 & ef & 29,66 & 0,77 & d & 25,74 & 2,06 & bcd & 61,43 & 1,71 & de \\
\hline 'Tadeo' & 4,97 & 0,24 & cde & 27,17 & 0,46 & d & 32,46 & 0,16 & d & 64,60 & 0,75 & e \\
\hline 'Dama Rosa' & 5,43 & 0,32 & def & 18,01 & 1,23 & c & 27,66 & 0,81 & $\mathrm{~cd}$ & 51,11 & 2,09 & bcd \\
\hline $\begin{array}{l}\text { 'Dama } \\
\text { Taronja' }\end{array}$ & 5,46 & 0,22 & def & 16,87 & 1,50 & bc & 17,88 & 0,75 & a & 40,21 & 2,46 & $a b$ \\
\hline 'GG9310' & 3,10 & 0,62 & $a b$ & 11,63 & 0,79 & a & 24,68 & 2,75 & $a b c$ & 39,41 & 3,73 & a \\
\hline 'GG979' & 4,92 & 0,60 & cde & 11,18 & 2,04 & a & 30,76 & 3,75 & $\mathrm{~cd}$ & 46,87 & 5,73 & $a b c$ \\
\hline 'GP9817' & 4,97 & 0,73 & cde & 16,54 & 0,06 & bc & 19,17 & 0,91 & $a b$ & 40,68 & 1,63 & $a b$ \\
\hline 'HG9821' & 6,62 & 0,30 & $\mathrm{f}$ & 13,37 & 1,83 & $a b$ & 31,16 & 0,08 & $\mathrm{~cd}$ & 51,15 & 1,70 & bcd \\
\hline 'HG9850' & 4,38 & 0,44 & bcd & 16,98 & 2,66 & $\mathrm{bc}$ & 31,56 & 5,08 & $\mathrm{~cd}$ & 52,93 & 7,61 & $\mathrm{~cd}$ \\
\hline 'HM964' & 2,99 & 0,44 & $a b$ & 12,96 & 1,43 & $a b$ & 24,36 & 2,68 & $a b c$ & 40,31 & 3,60 & $a b$ \\
\hline 'SEOP934' & 3,83 & 0,17 & $\mathrm{bc}$ & 18,23 & 1,09 & c & 24,62 & 2,53 & $a b c$ & 46,68 & 3,36 & $a b c$ \\
\hline
\end{tabular}

Regarding total sugar content in flesh, 'SEOP934' showed the highest value (12.34 g/100 g FW) and 'HG9850' the lowest one (5.75 g/100 g FW) (Figure S1A). In all cases sucrose was the predominant sugar, ranging from 65.1 to $90.3 \%$ of the total. For each sugar, 'Tadeo' showed the highest content of fructose $(0.48 \mathrm{~g} / 100 \mathrm{~g} \mathrm{FW})$ and glucose $(3.11 \mathrm{~g} / 100 \mathrm{~g} \mathrm{FW})$, and 'SEOP934' showed the highest quantity of sucrose (10.3 g/100 g FW). Regarding peel content, Kruskal-Wallis test showed an effect of the crop year over all the sugars analysed $(\alpha=0.05)$. According to the Spearman correlation analysis, 13 significant correlations were observed between the analysed sugars (Figure 6, Table S1). 


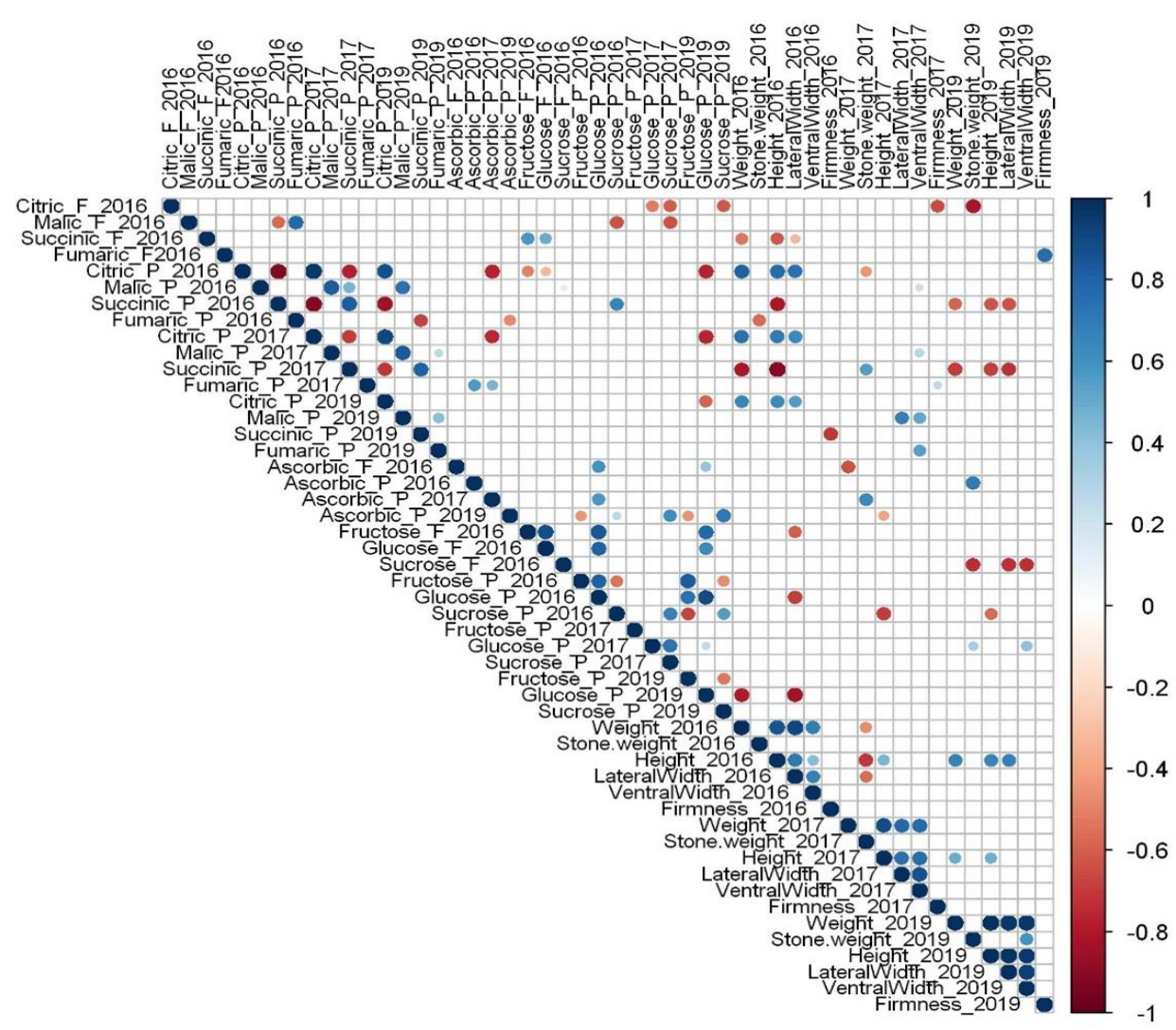

Figure 6. Significant correlations among variables analysed $(\alpha=0.05)$

Mainly, fructose and glucose appear positively correlated between tissues and also among years, while in peel fructose appeared negatively correlated with sucrose. The North American 'Goldrich' cultivar showed the lower total sugar content in 2016 (38.19 g/100 g DW) and the second lowest in 2017 (23.68 g/100 g DW), mainly due to its low sucrose content. In fact, this cultivar consistently showed the lowest sucrose contents (12.41, 4.63 and $17.80 \mathrm{~g} / 100 \mathrm{~g}$ DW, respectively). In general, the well-known cultivars from the Mediterranean Basin showed high sugar content and the accessions belonging to the IVIA's breeding program showed an intermediate content between them and 'Goldrich'. For each sugar, fructose ranged between the $3.65-26.60 \%$ of total sugar measured, glucose between $18.03-$ 49.09\% and sucrose between 19.57-72.70\%. As a measure of sweetness, SI and TSI index were calculated (Table S2). According to these indexes, fruits with identical total sugar content but with relatively more fructose or sucrose will taste sweeter. Overall, the Spanish cultivar 'Tadeo' and the selection of the breeding program 'HG9821' had the sweetest peel, while 'SEOP934' showed the sweetest flesh. Contrary, the selections 'Dama Rosa' and 'GG979' have the lower values in peel and 'HG9850' in flesh.

\section{I.4.2. Organic acids}

Significant differences among the apricot accessions were observed for citric, malic, succinic and fumaric acids content (Table 3, Figure S2). 
Table 3. Profiles of organic acids content in flesh (g/100 g FW) and peel (g /100 g DW) during 2016, 2017 and 2019.

\begin{tabular}{|c|c|c|c|c|c|c|c|c|c|c|c|c|c|c|c|}
\hline \multirow{2}{*}{$\begin{array}{c}\text { FLESH } 2016 \\
\text { Genotype }\end{array}$} & \multicolumn{3}{|c|}{ Citric } & \multicolumn{3}{|c|}{ Malic } & \multicolumn{3}{|c|}{ Succinic } & \multicolumn{3}{|c|}{ Fumaric } & \multicolumn{3}{|c|}{ Total } \\
\hline & Mean & SD & Significance & Mean & SD & Significance & Mean & SD & Significance & Mean & SD & Significance & Mean & SD & Significance \\
\hline 'Goldrich' & 1,496 & 0,040 & $\mathrm{~cd}$ & 1,431 & 0,023 & $\mathrm{~g}$ & 0,250 & 0,014 & bcd & 0,0029 & $3,962 \mathrm{E}-05$ & $\mathrm{~d}$ & 3,180 & 0,073 & $\mathrm{e}$ \\
\hline 'Canino' & 1,01 & 0,03 & $\mathrm{a}$ & 0,48 & 0,02 & c & 0,23 & 0,01 & $a b c$ & 0,0025 & 0,00008 & bcd & 1,73 & 0,07 & a \\
\hline 'Mitger' & 0,938 & 0,024 & a & 0,356 & 0,025 & $b$ & 0,266 & 0,013 & bcde & 0,0026 & $1,428 \mathrm{E}-04$ & $\mathrm{~cd}$ & 1,562 & 0,056 & a \\
\hline 'Tadeo' & 1,287 & 0,002 & $b$ & 0,526 & 0,005 & c & 0,540 & 0,029 & $f$ & 0,0025 & 1,551E-05 & $c d$ & 2,355 & 0,030 & $b$ \\
\hline 'Dama Rosa' & 1,626 & 0,054 & de & 1,305 & 0,034 & $f$ & 0,320 & 0,041 & cde & 0,0024 & $7,548 \mathrm{E}-05$ & $\mathrm{bc}$ & 3,254 & 0,115 & e \\
\hline 'Dama Taronja' & 1,874 & 0,038 & $f$ & 0,633 & 0,014 & $d$ & 0,292 & 0,054 & bcde & 0,0020 & $1,637 \mathrm{E}-04$ & $a b$ & 2,801 & 0,089 & $c d$ \\
\hline 'GG9310' & 1,731 & 0,064 & ef & 0,978 & 0,025 & e & 0,355 & 0,017 & $\mathrm{e}$ & 0,0034 & $2,186 \mathrm{E}-04$ & $\mathrm{e}$ & 3,067 & 0,098 & de \\
\hline 'GG979' & 1,512 & 0,058 & $\mathrm{~cd}$ & 0,475 & 0,024 & c & 0,248 & 0,005 & abcd & 0,0018 & 2,997E-04 & a & 2,236 & 0,084 & $b$ \\
\hline 'GP9817' & 1,828 & 0,027 & $f$ & 0,625 & 0,016 & $d$ & 0,344 & 0,069 & de & 0,0035 & 1,599E-04 & e & 2,800 & 0,108 & $c d$ \\
\hline 'HG9821' & 1,413 & 0,099 & $\mathrm{bc}$ & 1,019 & 0,083 & e & 0,256 & 0,036 & bcde & 0,0025 & $1,934 \mathrm{E}-04$ & $\mathrm{~cd}$ & 2,691 & 0,214 & c \\
\hline 'HG9850' & 1,065 & 0,032 & $\mathrm{a}$ & 0,363 & 0,017 & $b$ & 0,187 & 0,009 & $a b$ & 0,0020 & $5,038 \mathrm{E}-05$ & $a b$ & 1,617 & 0,057 & a \\
\hline 'HM964' & 1,434 & 0,033 & bc & 0,500 & 0,008 & c & 0,148 & 0,007 & $\mathrm{a}$ & 0,0022 & $1,365 \mathrm{E}-04$ & $a b c$ & 2,084 & 0,034 & $b$ \\
\hline 'SEOP934' & 1,890 & 0,066 & $f$ & 0,105 & 0,004 & $a$ & 0,342 & 0,018 & de & 0,0026 & $1,115 \mathrm{E}-04$ & $\mathrm{~cd}$ & 2,339 & 0,086 & $b$ \\
\hline PEEL 2016 & \multicolumn{3}{|c|}{ Citric } & \multicolumn{3}{|c|}{ Malic } & \multicolumn{3}{|c|}{ Succinic } & \multicolumn{3}{|c|}{ Fumaric } & \multicolumn{3}{|c|}{ Total } \\
\hline Genotype & Mean & SD & Significance & Mean & SD & Significance & Mean & SD & Significance & Mean & SD & Significance & Mean & SD & Significance \\
\hline 'Goldrich' & 10,860 & 1,485 & $\mathrm{~cd}$ & 8,449 & 0,342 & $\mathrm{~b}$ & 1,058 & 0,016 & $a b$ & 0,3365 & 0,032 & $b$ & 20,705 & 1,781 & $\mathrm{~b}$ \\
\hline 'Canino' & 9,58 & 1,04 & bcd & 5,48 & 2,28 & $a b$ & 1,73 & 0,14 & cde & 0,2754 & 0,03 & $a b$ & 17,07 & 3,30 & $b$ \\
\hline 'Mitger' & 1,414 & 0,428 & $a$ & 6,084 & 0,626 & $a b$ & 1,883 & 0,201 & efg & 0,2546 & 0,015 & $a$ & 9,636 & 1,255 & a \\
\hline 'Tadeo' & 2,013 & 0,118 & $a$ & 5,123 & 0,059 & $a b$ & 2,121 & 0,030 & $\mathrm{fg}$ & 0,2945 & 0,008 & $a b$ & 9,551 & 0,193 & a \\
\hline 'Dama Rosa' & 7,391 & 0,194 & $b$ & 6,973 & 0,146 & $a b$ & 1,839 & 0,023 & def & 0,2783 & 0,009 & $a b$ & 16,481 & 0,346 & $b$ \\
\hline 'Dama Taronja' & 11,630 & 1,537 & $d$ & 3,975 & 0,482 & $a$ & 1,505 & 0,096 & $\mathrm{~cd}$ & 0,2902 & 0,007 & $a b$ & 17,401 & 2,105 & $b$ \\
\hline 'GG9310' & 11,106 & 0,523 & $\mathrm{~cd}$ & 5,703 & 0,342 & $a b$ & 1,550 & 0,025 & cde & 0,2742 & 0,029 & $\mathrm{a}$ & 18,633 & 0,899 & $b$ \\
\hline 'GG979' & 11,705 & 0,111 & $\mathrm{~d}$ & 5,841 & 0,251 & $a b$ & 1,433 & 0,039 & $\mathrm{bc}$ & 0,2625 & 0,024 & $\mathrm{a}$ & 19,242 & 0,383 & $b$ \\
\hline 'GP9817' & 10,711 & 0,271 & $\mathrm{~cd}$ & 5,228 & 1,118 & $a b$ & 1,669 & 0,044 & cde & 0,2897 & 0,016 & $a b$ & 17,898 & 1,368 & $b$ \\
\hline 'HG9821' & 8,151 & 0,109 & $b$ & 6,759 & 0,556 & $a b$ & 1,623 & 0,004 & cde & 0,2585 & 0,011 & $\mathrm{a}$ & 16,792 & 0,657 & $b$ \\
\hline 'HG9850' & 9,217 & 0,473 & $\mathrm{bc}$ & 4,177 & 0,298 & a & 1,741 & 0,083 & cdef & 0,2676 & 0,014 & a & 15,403 & 0,286 & $b$ \\
\hline 'HM964' & 10,486 & 0,440 & $c d$ & 5,750 & 0,156 & $a b$ & 0,940 & 0,077 & a & 0,2893 & 0,021 & $a b$ & 17,465 & 0,668 & $b$ \\
\hline 'SEOP934' & 1,465 & 0,420 & $a$ & 14,195 & 3,401 & $\mathrm{C}$ & 2,257 & 0,307 & g & 0,2590 & 0,013 & $a$ & 18,177 & 4,019 & $b$ \\
\hline
\end{tabular}


Table 3. (Cont).

\begin{tabular}{|c|c|c|c|c|c|c|c|c|c|c|c|c|c|c|c|}
\hline \multirow{2}{*}{$\begin{array}{l}\text { PEEL } 2017 \\
\text { Genotype }\end{array}$} & \multicolumn{3}{|c|}{ Citric } & \multicolumn{3}{|c|}{ Malic } & \multicolumn{3}{|c|}{ Succinic } & \multicolumn{3}{|c|}{ Fumaric } & \multicolumn{3}{|c|}{ Total } \\
\hline & Mean & SD & Significance & Mean & SD & Significance & Mean & SD & Significance & Mean & SD & Significance & Mean & SD & Significance \\
\hline 'Goldrich' & 17,225 & 1,273 & $\mathrm{~cd}$ & 12,573 & 0,804 & $f$ & 1,625 & 0,090 & $a b$ & 0,2749 & 0,032 & $a$ & 31,698 & 2,163 & $\mathrm{e}$ \\
\hline 'Canino' & 8,922 & 0,489 & $b$ & 2,445 & 0,143 & a & 1,928 & 0,109 & bc & 0,2540 & 0,009 & $\mathrm{a}$ & 13,549 & 0,691 & $a b$ \\
\hline 'Mitger' & 1,795 & 0,064 & a & 7,667 & 0,074 & de & 2,530 & 0,011 & de & 0,4158 & 0,018 & $\mathrm{bc}$ & 12,407 & 0,030 & a \\
\hline 'Tadeo' & 2,171 & 0,213 & a & 6,476 & 0,343 & cde & 2,402 & 0,065 & $d$ & 0,2704 & 0,013 & a & 11,320 & 0,424 & a \\
\hline 'Dama Rosa' & 6,668 & 1,395 & $b$ & 8,155 & 1,881 & e & 2,347 & 0,180 & $d$ & 0,3435 & 0,098 & $a b c$ & 17,513 & 3,290 & $b$ \\
\hline 'Dama Taronja' & 17,602 & 2,128 & cde & 4,966 & 0,358 & $a b c$ & 1,431 & 0,020 & $a$ & 0,2712 & 0,011 & $\mathrm{a}$ & 24,270 & 2,376 & $\mathrm{~cd}$ \\
\hline 'GG9310' & 20,549 & 1,208 & e & 6,086 & 0,234 & bcde & 2,220 & 0,181 & $\mathrm{~cd}$ & 0,2748 & 0,010 & $a$ & 29,129 & 1,632 & de \\
\hline 'GG979' & 19,537 & 0,666 & de & 6,223 & 0,059 & bcde & 1,916 & 0,021 & bc & 0,2747 & 0,029 & a & 27,951 & 0,659 & de \\
\hline 'GP9817' & 14,595 & 0,602 & c & 5,456 & 0,160 & bcd & 1,941 & 0,098 & bc & 0,2800 & 0,031 & $a b$ & 22,273 & 0,885 & c \\
\hline 'HG9821' & 6,381 & 0,786 & $b$ & 3,880 & 0,479 & $a b$ & 1,394 & 0,143 & $a$ & 0,4540 & 0,021 & c & 12,109 & 1,154 & a \\
\hline 'HG9850' & 7,710 & 0,643 & $b$ & 2,469 & 0,033 & $a$ & 1,846 & 0,129 & bc & 0,4518 & 0,018 & c & 12,477 & 0,688 & $\mathrm{a}$ \\
\hline 'HM964' & 18,233 & 0,056 & de & 6,784 & 0,143 & cde & 2,155 & 0,073 & $\mathrm{~cd}$ & 0,2850 & 0,008 & $a b$ & 27,457 & 0,154 & cde \\
\hline 'SEOP934' & 1,273 & 0,033 & a & 21,244 & 0,237 & $\mathrm{~g}$ & 2,871 & 0,178 & e & 0,2648 & 0,008 & a & 25,652 & 0,182 & $\mathrm{~cd}$ \\
\hline PEEL 2019 & \multicolumn{3}{|c|}{ Citric } & \multicolumn{3}{|c|}{ Malic } & \multicolumn{3}{|c|}{ Succinic } & \multicolumn{3}{|c|}{ Fumaric } & \multicolumn{3}{|c|}{ Total } \\
\hline Genotype & Mean & sd & Significance & Mean & sd & Significance & Mean & sd & Significance & Mean & sd & Significance & Mean & sd & Significance \\
\hline 'Goldrich' & 28,358 & 0,524 & $\mathrm{fg}$ & 13,394 & 0,262 & $\mathrm{~g}$ & 0,814 & 0,031 & $\mathrm{a}$ & 0,000 & 0,000 & $\mathrm{a}$ & 42,566 & 0,805 & g \\
\hline 'Canino' & 12,930 & 3,091 & bc & 2,188 & 0,051 & a & 0,823 & 0,156 & a & 0,002 & 0,000 & $a b$ & 15,944 & 2,890 & $\mathrm{~b}$ \\
\hline 'Mitger' & 0,758 & 0,026 & $a$ & 10,334 & 0,189 & $f$ & 3,175 & 0,137 & e & 0,014 & 0,000 & $f$ & 14,280 & 0,350 & $a b$ \\
\hline 'Tadeo' & 3,233 & 0,059 & a & 6,193 & 0,054 & c & 1,332 & 0,025 & $b$ & 0,006 & 0,001 & bc & 10,763 & 0,136 & a \\
\hline 'Dama Rosa' & 16,178 & 1,530 & c & 10,375 & 0,986 & $f$ & 2,742 & 0,244 & $d$ & 0,011 & 0,001 & ef & 29,306 & 2,760 & $d$ \\
\hline 'Dama Taronja' & 30,924 & 0,687 & gh & 6,502 & 0,147 & $\mathrm{~cd}$ & 1,190 & 0,065 & $b$ & 0,033 & 0,002 & $\mathrm{~h}$ & 38,649 & 0,881 & $\mathrm{fg}$ \\
\hline 'GG9310' & 33,047 & 0,411 & $\mathrm{~h}$ & 6,268 & 0,085 & c & 2,646 & 0,035 & $d$ & 0,005 & 0,000 & $\mathrm{bc}$ & 41,967 & 0,529 & $\mathrm{~g}$ \\
\hline 'GG979' & 24,570 & 1,128 & de & 6,295 & 0,242 & C & 2,460 & 0,133 & $d$ & 0,027 & 0,002 & $\mathrm{~g}$ & 33,352 & 1,504 & de \\
\hline 'GP9817' & 26,035 & 0,741 & def & 7,509 & 0,183 & de & 1,743 & 0,053 & c & 0,007 & 0,000 & $\mathrm{~cd}$ & 35,294 & 0,975 & ef \\
\hline 'HG9821' & 11,196 & 0,286 & $b$ & 7,887 & 0,274 & e & 1,112 & 0,042 & $a b$ & 0,005 & 0,001 & $\mathrm{bc}$ & 20,200 & 0,589 & c \\
\hline 'HG9850' & 23,822 & 0,795 & $d$ & 4,881 & 0,177 & $b$ & 1,282 & 0,029 & $b$ & 0,006 & 0,001 & $b c$ & 29,990 & 1,000 & $d$ \\
\hline 'HM964' & 27,341 & 0,257 & ef & 6,639 & 0,065 & $\mathrm{~cd}$ & 1,374 & 0,042 & $b$ & 0,010 & 0,000 & de & 35,364 & 0,338 & ef \\
\hline 'SEOP934' & 2,684 & 0,044 & $\mathrm{a}$ & 26,402 & 0,346 & $\mathrm{~h}$ & 3,535 & 0,033 & $f$ & 0,054 & 0,000 & i & 32,675 & 0,396 & de \\
\hline
\end{tabular}


In this case, 21 significant correlations were detected between the organics analyzed, being the most notorious the negative correlation between succinic and citric acids in peel (Figure 6, Table S1). In flesh, citric acid was the main organic acid in all cases, ranging from 47-80.8\%. Malic acid was the second one, ranging from 4.5-45\%, except for 'Tadeo' ( 22.2\%), which showed more succinic content (22.9\%). Succinic represented between 7.1-22.93\% of the organic acids measured and fumaric just between 0.07 and $0.17 \%$. Regarding total content in flesh, 'Dama Rosa' showed the highest value $(3.254 \mathrm{~g} / 100$ g FW) and 'Mitger' the lower one (1.562 g/100 g FW) (Table 2, Figure S2). As in the case of sugars, an effect of crop year was observed over the peel content in all the organic acids analysed $(\alpha=0.05)$. The content of fumaric was especially low in 2019, which was confirmed by repeating the analyzes. Regarding peel content, citric acid was the main organic acid in all cases except for 'SEOP934' (6\%), 'Mitger' (12.5\%) and 'Tadeo' (23.4\%), which consistently showed a higher content of malic acid (77.8\%, $68.6 \%$ and $56.1 \%$, respectively) and also succinic acid (with mean values of $15.3 \%, 17 \%$ and $18.60 \%$, respectively), except 'Tadeo' in 2019. Regarding the total content in peel, 'Goldrich' showed the highest values (20.7, 31.7 and $42.6 \mathrm{~g} / 100 \mathrm{~g}$ DW), and 'Tadeo' the lower ones (9.6, 11.3 and 10.8 g/100 g DW) during the three years analysed.

\section{I.4.3. Ascorbic acid}

Results of ascorbic acid content in peel and flesh of the genotypes studied in the 3 crop years are in Table 4 and Figure S3. Significant differences were found among crop years $(\alpha=0.05)$. In flesh, values ranged from $9.11 \mathrm{mg} / 100 \mathrm{~g} \mathrm{FW}$ ('SEOP934') and 13.08 mg/100 g FW ('HG9821'). Regarding peel content, 'Mitger', 'HG9850' and 'HM964' showed the highest values in 2016 (185.02 mg/100 g DW), 2017 (192.82 mg/100 g DW) and 2019 (165.16 mg/100 g DW), respectively.

Table 4. Ascorbic acid content in flesh (mg/100 g FW) and peel (g /100 g DW) during 2016, 2017 and 2019.

\begin{tabular}{|c|c|c|c|c|c|c|c|c|c|c|c|c|}
\hline \multirow[b]{2}{*}{ Genotype } & \multicolumn{3}{|c|}{ Flesh 2016} & \multicolumn{3}{|c|}{ Peel 2016} & \multicolumn{3}{|c|}{ Peel 2017} & \multicolumn{3}{|c|}{ Peel 2019} \\
\hline & Mean & SD & Sig. & Mean & SD & Sig. & Mean & SD & Sig. & Mean & SD & Sig. \\
\hline 'Goldrich' & 10,36 & 0,74 & $a b c$ & 178,29 & 25,03 & ef & 139,29 & 5,77 & $a b$ & 66,84 & 3,06 & $a$ \\
\hline 'Canino' & 9,40 & 0,90 & $a$ & 120,84 & 2,22 & $a b c$ & 102,94 & 5,11 & a & 122,70 & 20,48 & c \\
\hline 'Mitger' & 11,80 & 0,50 & $a b c$ & 185,02 & 5,17 & $f$ & 174,60 & 3,57 & bc & 105,83 & 0,86 & bc \\
\hline 'Tadeo' & 13,02 & 0,20 & bc & 121,12 & 5,94 & $a b c$ & 170,52 & 17,80 & bc & 98,44 & 1,15 & bc \\
\hline ‘D. Rosa' & 11,51 & 0,31 & $a b c$ & 144,00 & 7,38 & bcde & 161,95 & 18,18 & bc & 93,84 & 8,89 & $a b c$ \\
\hline 'D. Taronja' & 10,25 & 0,62 & $a b c$ & 111,20 & 8,05 & $a b$ & 138,42 & 7,64 & $a b$ & 93,87 & 3,35 & $a b c$ \\
\hline 'GG9310' & 9,92 & 0,30 & $a b$ & 128,54 & 5,02 & abcd & 139,13 & 14,10 & $a b$ & 102,64 & 19,00 & $c$ \\
\hline 'GG979' & 10,50 & 2,30 & $a b c$ & 148,44 & 8,01 & bcdef & 127,38 & 12,81 & a & 109,38 & 4,51 & $b c$ \\
\hline 'GP9817' & 11,97 & 0,83 & $a b c$ & 111,61 & 25,39 & $a b$ & 140,26 & 9,36 & $a b$ & 93,66 & 0,76 & $a b c$ \\
\hline 'HG9821' & 13,08 & 0,29 & c & 98,70 & 11,25 & a & 192,43 & 8,53 & c & 85,51 & 1,55 & $a b$ \\
\hline 'HG9850' & 11,43 & 1,03 & $a b c$ & 165,64 & 22,30 & def & 192,82 & 2,25 & c & 101,23 & 18,82 & $b c$ \\
\hline 'HM964' & 9,57 & 0,19 & a & 159,49 & 1,71 & cdef & 177,65 & 15,68 & c & 165,16 & 3,35 & $\mathrm{~d}$ \\
\hline 'SEOP934' & 9,11 & 1,86 & a & 137,78 & 2,14 & abcde & 175,55 & 10,83 & $\mathrm{bc}$ & 90,72 & 0,71 & $a b$ \\
\hline
\end{tabular}

Sig.: Significance; SD: Standard Deviation. 


\section{I.4.4. Pomological traits}

Ten fruit traits, mainly related with size, firmness, and colour, were studied in the 3 crop years and significant differences were found among crop years and genotypes ( $\alpha=0.05)$ (Table 5). 'Dama Taronja' in 2016 and 2019 and 'HG9821' in 2017 showed the highest weight, almost 3 times higher than the lowest one in all cases. Fruit colour was also influenced by the environment, with slight variations observed every year. Anyway, 'Dama Taronja', 'HM964' and 'SEOP934' showed medium to dark orange flesh colour, that could point them as good carotenoid sources. Regarding firmness, another trait highly affected during the ripening process, significant differences were also observed between the analysed accession, showing in general higher values the traditional cultivars, like 'Goldrich', 'Canino', 'Mitger' and 'Tadeo'. 
Table 5. Pomological traits measured during 2016, 2017 and 2019.

\begin{tabular}{|c|c|c|c|c|c|c|c|c|c|c|c|c|c|c|c|c|c|c|c|c|c|c|c|c|}
\hline \multirow{2}{*}{ Genotype } & \multirow[t]{2}{*}{ Year } & \multirow{2}{*}{$\begin{array}{l}\text { Harvest } \\
\text { date }\end{array}$} & \multicolumn{3}{|c|}{ Weight (g) } & \multicolumn{3}{|c|}{ Stone weight (g) } & \multicolumn{3}{|c|}{ Height (mm) } & \multicolumn{3}{|c|}{$\begin{array}{l}\text { Lateral Width } \\
(\mathrm{mm})\end{array}$} & \multicolumn{3}{|c|}{$\begin{array}{l}\begin{array}{l}\text { Ventral Width } \\
(\mathrm{mm})\end{array} \\
\end{array}$} & \multicolumn{3}{|c|}{$\begin{array}{c}\text { Firmness } \\
(\mathrm{kgf} / \mathrm{cm} 2)\end{array}$} & \multirow{2}{*}{$\begin{array}{l}\text { Skin } \\
\text { Color* }\end{array}$} & \multirow{2}{*}{$\begin{array}{l}\text { Over } \\
\text { Color }\end{array}$} & \multirow{2}{*}{$\begin{array}{l}\text { Intensity } \\
\text { Over } \\
\text { Color* }\end{array}$} & \multirow{2}{*}{$\begin{array}{l}\text { Flesh } \\
\text { Color* }\end{array}$} \\
\hline & & & mean & SD & Sig. & mean & SD & Sig. & mean & SD & Sig. & mean & SD & Sig. & mean & SD & Sig. & mean & SD & Sig. & & & & \\
\hline ‘Goldrich’ & 2016 & 22/Jun & 56,25 & 3,19 & c & 13,03 & 0,91 & a & 46,79 & 3,20 & $\mathrm{bcd}$ & 46,06 & 1,27 & bc & 42,82 & 0,46 & $\mathrm{~b}$ & 2,53 & 0,89 & ef & M orange & $\begin{array}{c}\text { orange } \\
\text { red }\end{array}$ & $\mathrm{L}$ & D orange \\
\hline 'Canino & 2016 & 03/Jun & 63,32 & 4,92 & bc & 17,54 & 1,52 & bc & 47,31 & 1,79 & c & 48,54 & 1,52 & $c d$ & 44,43 & 1,79 & c & 3,65 & 0,97 & $f$ & $\begin{array}{l}\text { yellow } \\
\text { green }\end{array}$ & $\begin{array}{l}\text { orange } \\
\text { red }\end{array}$ & $\mathrm{L}$ & cream \\
\hline 'Mitger' & 2016 & 03/Jun & 50,64 & 6,29 & c & 18,72 & 1,57 & bc & 41,79 & 1,51 & $b$ & 45,74 & 1,68 & $\mathrm{bc}$ & 47,46 & 2,11 & de & 2,49 & 0,75 & ef & yellowish & pink & D & cream \\
\hline 'Tadeo' & 2016 & 15/Jun & 30,70 & 2,13 & e & 18,11 & 1,76 & c & 36,16 & 2,22 & a & 38,95 & 1,33 & a & 38,72 & 1,29 & a & 3,00 & 0,78 & $f$ & $\begin{array}{l}\text { yellow } \\
\text { green }\end{array}$ & $\begin{array}{l}\text { orange } \\
\text { red }\end{array}$ & M & L orange \\
\hline $\begin{array}{l}\text { 'Dama } \\
\text { Rosa' }\end{array}$ & 2016 & 06/Jun & 51,92 & 7,09 & $\mathrm{~cd}$ & 16,76 & 3,64 & $\mathrm{bc}$ & 42,21 & 2,82 & $\mathrm{~b}$ & 47,72 & 2,31 & bcd & 42,73 & 2,32 & bc & 1,42 & 0,50 & cde & $\begin{array}{l}\text { yellow } \\
\text { green }\end{array}$ & pink & D & L orange \\
\hline $\begin{array}{l}\text { 'Dama } \\
\text { Taronja' }\end{array}$ & 2016 & 10/Jun & 93,40 & 21,31 & $a$ & 20,06 & 8,19 & abcde & 55,83 & 4,53 & $d$ & 53,78 & 5,01 & cde & 51,95 & 3,86 & $\mathrm{fg}$ & 2,88 & 0,96 & ef & $\begin{array}{l}\text { yellow } \\
\text { green }\end{array}$ & $\begin{array}{l}\text { orange } \\
\text { red }\end{array}$ & L & D orange \\
\hline 'GG9310' & 2016 & 06/Jun & 62,00 & 6,85 & $b c$ & 23,68 & 2,64 & cde & 44,75 & 2,84 & c & 47,75 & 1,83 & bcd & 46,71 & 4,15 & def & 0,45 & 0,10 & a & $\begin{array}{l}\text { yellow } \\
\text { green }\end{array}$ & $\begin{array}{l}\text { orange } \\
\text { red }\end{array}$ & L & cream \\
\hline 'GG979' & 2016 & 13/Jun & 77,34 & 5,06 & $a$ & 18,80 & 2,16 & bc & 46,61 & 2,39 & c & 52,48 & 1,92 & e & 51,21 & 1,41 & $\mathrm{~g}$ & 1,11 & 0,89 & $\mathrm{bc}$ & yellowish & pink & M & L orange \\
\hline 'GP9817' & 2016 & 13/Jun & 61,86 & 12,62 & $\mathrm{bc}$ & 18,15 & 3,10 & bc & 43,83 & 2,98 & $b c$ & 50,41 & 4,16 & de & 46,92 & 3,78 & cde & 1,44 & 0,79 & abcde & $\begin{array}{l}\text { yellow } \\
\text { green }\end{array}$ & red & $M$ & L orange \\
\hline 'HG9821' & 2016 & 08/Jun & 73,62 & 12,94 & $a$ & 27,36 & 7,76 & e & 45,69 & 4,99 & $\mathrm{bcd}$ & 50,86 & 6,59 & de & 48,41 & 2,80 & e & 1,26 & 0,52 & $d$ & $\begin{array}{l}\text { yellow } \\
\text { green }\end{array}$ & pink & $M$ & cream \\
\hline 'HG9850' & 2016 & 03/Jun & 70,30 & 17,11 & $b$ & 23,52 & 4,00 & cde & 46,45 & 4,28 & bc & 48,08 & 0,64 & $c d$ & 48,05 & 1,11 & ef & 1,79 & 0,41 & e & $\begin{array}{l}\text { yellow } \\
\text { green }\end{array}$ & pink & L & $\begin{array}{l}\text { cream } / \mathrm{L} \\
\text { orange }\end{array}$ \\
\hline 'HM964' & 2016 & 01/Jun & 43,88 & 5,77 & $\mathrm{~cd}$ & NA & NA & & 36,34 & 1,86 & a & 43,66 & 2,27 & $b$ & 43,29 & 2,22 & bc & 0,73 & 0,09 & $b$ & $\begin{array}{l}\text { yellow } \\
\text { green }\end{array}$ & $\begin{array}{l}\text { orange } \\
\mathrm{red} / \mathrm{red}\end{array}$ & L-M & $\begin{array}{l}\text { cream } / L \\
\text { orange }\end{array}$ \\
\hline 'SEOP934' & 2016 & 08/Jun & 51,11 & 2,89 & $\mathrm{~cd}$ & 22,22 & 2,32 & $d$ & 39,09 & 4,49 & $\mathrm{a}$ & 47,01 & 0,77 & c & 46,71 & 0,63 & de & 0,94 & 0,25 & $\mathrm{bc}$ & L orange & pink & $M$ & $M$ orange \\
\hline
\end{tabular}

* L: ligth; M: medium; D: dark.

Sig.: Significance; SD: Standard Deviation. 
Table 5. (Cont.)

\begin{tabular}{|c|c|c|c|c|c|c|c|c|c|c|c|c|c|c|c|c|c|c|c|c|c|c|c|c|}
\hline \multirow[t]{2}{*}{ Genotype } & \multirow{2}{*}{ Year } & \multirow{2}{*}{$\begin{array}{c}\text { Harvest } \\
\text { date }\end{array}$} & \multicolumn{3}{|c|}{ Weight (g) } & \multicolumn{3}{|c|}{ Stone weight (g) } & \multicolumn{3}{|c|}{ Height (mm) } & \multicolumn{3}{|c|}{$\begin{array}{l}\text { Lateral Width } \\
(\mathrm{mm})\end{array}$} & \multicolumn{3}{|c|}{$\begin{array}{c}\text { Ventral Width } \\
(\mathrm{mm})\end{array}$} & \multicolumn{3}{|c|}{ Firmness (kgf/cm2) } & \multirow[t]{2}{*}{ Skin Color* } & \multirow{2}{*}{$\begin{array}{l}\text { Over } \\
\text { Color }\end{array}$} & \multirow{2}{*}{$\begin{array}{c}\text { Intensity } \\
\text { Over } \\
\text { Color* }\end{array}$} & \multirow{2}{*}{$\begin{array}{l}\text { Flesh } \\
\text { Color* }\end{array}$} \\
\hline & & & mean & SD & Sig. & mean & SD & Sig. & mean & SD & Sig. & mean & SD & Sig. & mean & SD & Sig. & mean & SD & Sig. & & & & \\
\hline ‘Goldrich' & 2017 & 09/Jun & 50,87 & 3,96 & $\mathrm{~cd}$ & 14,80 & 2,21 & abcd & 47,35 & 1,76 & e & 43,86 & 1,47 & $a b$ & 39,91 & 1,54 & bc & 3,55 & 1,53 & $a b c$ & M orange & $\begin{array}{l}\text { orange } \\
\text { red }\end{array}$ & M & M orange \\
\hline 'Canino' & 2017 & 31/May & 35,77 & 6,19 & $\mathrm{bc}$ & 11,64 & 1,11 & a & 36,16 & 3,11 & $a b c$ & 37,27 & 6,10 & a & 34,47 & 2,27 & $a b$ & 3,85 & 1,41 & $a b c$ & L orange & pink & L & L orange \\
\hline 'Mitger' & 2017 & 25/May & 35,23 & 6,31 & bc & 15,13 & 1,92 & bc & 38,94 & 1,93 & c & 42,03 & 1,12 & a & 40,70 & 1,67 & c & 3,11 & 0,80 & b & yellowish & purple & D & $\begin{array}{l}\text { withish } \\
\text { green }\end{array}$ \\
\hline 'Tadeo' & 2017 & 09/Jun & 23,20 & 2,52 & a & 20,86 & 6,41 & abcde & 33,93 & 1,57 & $a b$ & 36,94 & 2,49 & a & 32,17 & 2,19 & a & 3,08 & 1,22 & $a b c$ & yellowish & pink & D & cream \\
\hline $\begin{array}{l}\text { 'Dama } \\
\text { Rosa' }\end{array}$ & 2017 & 09/Jun & 46,53 & 3,95 & c & 14,91 & 1,95 & $a b c$ & 40,93 & 0,90 & $\mathrm{~cd}$ & 45,57 & 0,65 & b & 39,37 & 1,29 & bc & 2,04 & 0,37 & b & $\begin{array}{l}\text { yellow } \\
\text { green }\end{array}$ & pink & D & cream \\
\hline $\begin{array}{l}\text { 'Dama } \\
\text { Taronja' }\end{array}$ & 2017 & 09/Jun & 49,30 & 7,64 & $\mathrm{~cd}$ & 10,51 & 2,92 & $a b c$ & 45,75 & 0,69 & e & 45,35 & 3,20 & $a b c$ & 41,29 & 1,30 & abcd & 0,59 & 0,09 & a & D orange & $\begin{array}{l}\text { orange } \\
\text { red }\end{array}$ & M & D orange \\
\hline 'GG9310' & 2017 & 09/Jun & 38,87 & 9,48 & $a b c$ & 17,03 & 4,88 & abcd & 37,93 & 2,74 & $a b c d$ & 40,63 & 4,75 & $a b$ & 35,53 & 0,89 & a & 0,97 & 0,27 & a & yellowish & $\begin{array}{l}\text { orange } \\
\text { red }\end{array}$ & L & L orange \\
\hline 'GG979' & 2017 & 09/Jun & 44,87 & 5,62 & $\mathrm{~cd}$ & 15,18 & 3,11 & abcd & 39,32 & 4,60 & bcde & 40,78 & 2,49 & $a b$ & 38,55 & 1,44 & $a b c$ & 1,02 & 0,39 & $a b c$ & L orange & pink & $\mathrm{D}$ & L orange \\
\hline 'GP9817' & 2017 & 09/Jun & 32,80 & 1,73 & b & 13,12 & 0,69 & $a b$ & 35,96 & 1,05 & bc & 42,03 & 0,26 & a & 35,88 & 1,53 & ab & 3,27 & 1,61 & $a b c$ & $\begin{array}{l}\text { yellow } \\
\text { green }\end{array}$ & pink & M & cream \\
\hline 'HG9821' & 2017 & $25 / J u n$ & 83,40 & 1,27 & e & 27,35 & 0,07 & e & NA & NA & & NA & NA & & NA & NA & & NA & NA & & L orange & pink & M & cream \\
\hline 'HG9850' & 2017 & $25 / J u n$ & 48,83 & 5,09 & $\mathrm{~cd}$ & 18,19 & 2,70 & $c d$ & 41,60 & 1,19 & $d$ & 45,09 & 1,39 & $b$ & 41,74 & 2,01 & c & 6,80 & 1,49 & c & yellowish & pink & $M$ & cream \\
\hline 'HM964' & 2017 & 02/Jun & 33,43 & 2,30 & b & 14,39 & 0,99 & b & 33,37 & 0,60 & a & 41,47 & 1,28 & a & 39,63 & 1,89 & bc & 2,52 & 0,41 & b & $\begin{array}{l}\text { yellow } \\
\text { green }\end{array}$ & pink & M & white \\
\hline 'SEOP934' & 2017 & 02/Jun & 56,50 & 3,32 & $d$ & 20,20 & 1,43 & $d$ & 40,24 & 1,57 & $c d$ & 48,17 & 1,74 & c & 47,36 & 1,41 & d & 1,62 & 0,86 & $a b c$ & yellowish & pink & M & L orange \\
\hline
\end{tabular}

* L: ligth; M: medium; D: dark.

Sig.: Significance; SD: Standard Deviation. 
Table 5. (Cont.)

\begin{tabular}{|c|c|c|c|c|c|c|c|c|c|c|c|c|c|c|c|c|c|c|c|c|c|c|c|c|}
\hline \multirow[t]{2}{*}{ Genotype } & \multirow{2}{*}{ Year } & \multirow{2}{*}{$\begin{array}{c}\text { Harvest } \\
\text { date }\end{array}$} & \multicolumn{3}{|c|}{ Weight (g) } & \multicolumn{3}{|c|}{ Stone weight (g) } & \multicolumn{3}{|c|}{ Height (mm) } & \multicolumn{3}{|c|}{ Lateral Width $(\mathrm{mm})$} & \multicolumn{3}{|c|}{$\begin{array}{l}\text { Ventral Width } \\
(\mathrm{mm})\end{array}$} & \multicolumn{3}{|c|}{ Firmness (kgf/cm2) } & \multirow{2}{*}{$\begin{array}{l}\text { Skin } \\
\text { Color* }\end{array}$} & \multirow{2}{*}{$\begin{array}{l}\text { Over } \\
\text { Color }\end{array}$} & \multirow{2}{*}{$\begin{array}{c}\text { Intensity } \\
\text { Over } \\
\text { Color* }\end{array}$} & \multirow{2}{*}{$\begin{array}{l}\text { Flesh } \\
\text { Color* }\end{array}$} \\
\hline & & & mean & SD & Sig. & mean & SD & Sig. & mean & SD & Sig. & mean & SD & Sig. & mean & SD & Sig. & mean & SD & Sig. & & & & \\
\hline ‘Goldrich’ & 2019 & $19 /$ Jun & 77,95 & 14,67 & $c d$ & 20,81 & 3,42 & b & 54,88 & 1,42 & a & 51,47 & 3,46 & c & 46,99 & 3,02 & $\mathrm{bc}$ & 2,06 & 1,56 & bcd & M orange & red & M & M orange \\
\hline 'Canino' & 2019 & $11 /$ Jun & 52,15 & 5,44 & d & 17,23 & 2,93 & b & 42,43 & 0,80 & b & 44,71 & 2,25 & $b c$ & 42,69 & 1,58 & $\mathrm{~b}$ & 0,47 & 0,09 & a & M orange & red & D & $M$ orange \\
\hline 'Mitger' & 2019 & $11 /$ Jun & 64,85 & 12,30 & $\mathrm{~cd}$ & 28,13 & 4,71 & c & 45,51 & 3,01 & $b$ & 49,04 & 3,56 & c & 50,50 & 2,50 & c & 2,28 & 2,19 & abcde & yellowish & purple & M & white \\
\hline 'Tadeo' & 2019 & 19/Jun & 41,75 & 8,77 & $a b c$ & 19,88 & 4,18 & b & 39,13 & 2,19 & $b c$ & 43,40 & 2,46 & a & 41,92 & 3,71 & $a b$ & 1,93 & 1,79 & abcde & yellowish & pink & $M-D$ & cream \\
\hline $\begin{array}{l}\text { 'Dama } \\
\text { Rosa' }\end{array}$ & 2019 & $05 /$ Jun & 32,25 & 5,77 & $a b$ & 13,59 & 1,81 & $a b$ & 36,50 & 2,51 & $\mathrm{~cd}$ & 39,53 & 2,62 & $a b$ & 36,39 & 2,67 & a & 1,48 & 0,32 & cde & L orange & pink & D & L orange \\
\hline $\begin{array}{c}\text { 'Dama } \\
\text { Taronja' }\end{array}$ & 2019 & 05/Jun & 84,05 & 10,35 & $d$ & 13,15 & 1,01 & $a b$ & 53,03 & 2,88 & $a$ & 51,29 & 1,72 & c & 49,71 & 2,45 & c & 1,47 & 1,15 & bcd & M orange & pink & D & D orange \\
\hline 'GG9310' & 2019 & 29/May & 45,30 & 5,56 & $\mathrm{bc}$ & 17,73 & 1,04 & b & 39,14 & 3,57 & $b c$ & 44,36 & 1,30 & $b c$ & 41,97 & 2,56 & $a b$ & 1,89 & 0,34 & $d$ & L orange & $\begin{array}{l}\text { orange } \\
\text { red }\end{array}$ & L & L orange \\
\hline 'GG979' & 2019 & $17 /$ Jun & 55,35 & 10,46 & bc & 18,45 & 3,49 & b & 43,42 & 2,06 & $\mathrm{~b}$ & 45,78 & 2,84 & $\mathrm{bc}$ & 44,69 & 3,21 & $\mathrm{bc}$ & 0,74 & 0,51 & $a b c$ & L orange & pink & $M$ & L orange \\
\hline 'GP9817' & 2019 & $11 /$ Jun & 40,05 & 2,90 & $b$ & 14,49 & 1,38 & $a b$ & 38,25 & 1,09 & $c$ & 44,50 & 1,27 & bc & 40,80 & 1,58 & $a b$ & 4,42 & 1,14 & e & $\begin{array}{l}\text { yellow } \\
\text { green }\end{array}$ & $\begin{array}{l}\text { orange } \\
\text { red }\end{array}$ & $M$ & cream \\
\hline 'HG9821' & 2019 & 04/Jun & 46,75 & 7,76 & bc & 21,17 & 3,75 & $b$ & 41,06 & 2,83 & bc & 48,03 & 3,66 & $\mathrm{bc}$ & 41,78 & 1,87 & $b$ & 0,78 & 0,05 & $b$ & $\begin{array}{l}\text { yellow } \\
\text { green }\end{array}$ & pink & D & L orange \\
\hline 'HG9850' & 2019 & 23/May & 59,55 & 6,56 & c & 20,12 & 1,18 & b & 43,42 & 2,28 & $\mathrm{~b}$ & 47,68 & 2,09 & c & 45,17 & 2,94 & $\mathrm{bc}$ & 1,62 & 0,65 & $d$ & $M$ orange & pink & D & M orange \\
\hline 'HM964' & 2019 & 27/May & 29,70 & 2,69 & $\mathrm{a}$ & 15,14 & 2,45 & $a b$ & 32,94 & 1,09 & $d$ & 38,09 & 2,48 & a & 37,67 & 1,21 & a & 2,46 & 0,81 & de & L orange & $\begin{array}{l}\text { orange } \\
\text { red }\end{array}$ & M & cream \\
\hline 'SEOP934' & 2019 & $03 /$ Jun & 31,33 & 2,67 & $\mathrm{a}$ & 12,58 & 1,58 & a & 32,00 & 1,25 & $d$ & 38,85 & 1,37 & a & 38,89 & 1,21 & $a b$ & 1,11 & 0,21 & c & $\begin{array}{l}\text { yellow } \\
\text { green }\end{array}$ & pink & D & L orange \\
\hline
\end{tabular}

* L: ligth; M: medium; D: dark.

Sig.: Significance; SD: Standard Deviation. 


\section{I.4.5. Correlations and Principal Component Analysis}

As consumer preferences are highly influenced by the balance of sugar and organic acids content, relations between all the analysed compounds were also studied (Figure 6, Table S1). As 'HG9821' and 'HM964' had some pomological data missing, these accessions were eliminated from the analysis. Emphasizing just the strongest correlations $(-0.8>x>0.8)$, malic content in flesh was highly and positively correlated with fumaric content in peel, while citric content in peel was highly and negatively correlated with succinic, ascorbic acid and glucose content. Glucose and fructose showed positive correlations in both tissues. Regarding pomological traits, fruit weight and height showed positive correlations with citric content but negatively with succinic and glucose content.

In order to explore the variability observed in the accessions, the pomological and nutraceutical data for each year were submitted to principal component analyses (PCA). As results with each independent data set were quite similar, just the PCA for 2017 is shown (Figure 7).
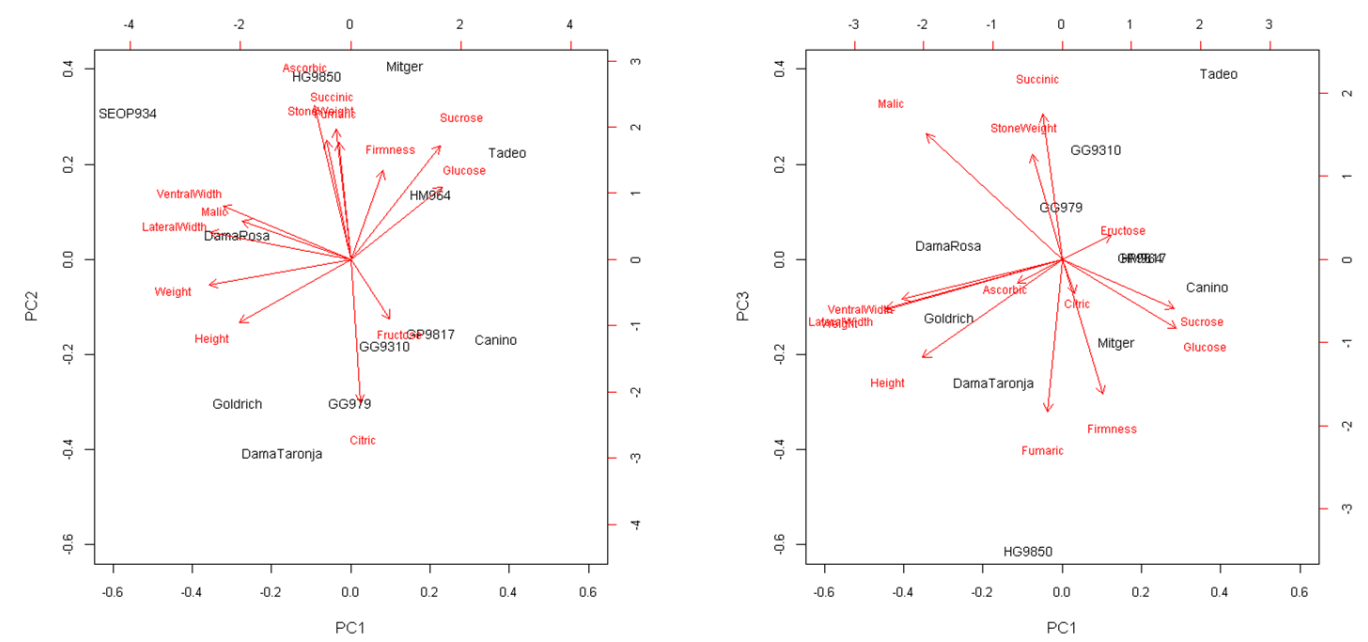

Figure 7. Principal Component Analysis for 2017 for pomological and nutraceutical data. Left: first and second components; Right: first and third components

First 3 principal components (PC1, PC2 and PC3) accounted for $73.8 \%$ of the total variance $(31.06 \%$, $27.29 \%$ and $15.45 \%$, respectively). PC1 was positively correlated mainly with sugar content and firmness, and negatively with malic content and fruit size traits. PC2 showed a positive correlation mainly with succinic, fumaric, ascorbic acids, sucrose, stone weight and firmness, and negatively with citric acid and fructose content. PC3 showed a positive correlation with succinic, malic, and stone weight, but in this case a negative one with firmness and fumaric. Accessions appear distributed in the space of the 3 first components without a clear substructure. 'Goldrich' and 'Dama Taronja' appeared close to each other, but the other 'Goldrich' descendants appear more separated by the PC3. 'Canino' and 'GP9817' appeared also close to each other, while 'Tadeo' appears clearly separated from the rest. 


\section{I.5. Discussion}

Traditionally, plant breeding goals have been focused on yield, stress resistance and external quality traits as appearance and shelf-life. However, consumers are increasingly demanding high quality food. As an example, huge efforts are in progress to recover the lost flavour in tomato cultivars (Tieman et al., 2017). Nowadays, internal quality traits have been incorporated as objectives of almost any plant breeding program. Great efforts are being made in order to identify genes of interest involved in the control of these traits that could be useful to facilitate breeding programs (García-Gómez, 2019; Zhang et al., 2019). The IVIA's apricot breeding program started in 1993 and was initially focused on introgression of sharka resistance into locally grown cultivars (Martínez-Calvo et al., 2009). However, just a handful of North American apricot PPV resistant cultivars, adapted to cold-growing conditions, have been identified (Martínez-Gómez et al., 2000). Despite the crosses with those cultivars introduce also undesirable traits, the hybrids obtained in the breeding program represent a good opportunity to incorporate new breeding goals and to accelerate the development of new varieties better adapted to the Mediterranean basin conditions. In this sense, the characterization of the nutraceutical properties of these germplasm collection allows to identify putative promising accessions and to optimize the design of the future crosses. This study opens also future work to study the genetic control of these traits in apricot. In this work, we analysed 13 accessions of the IVIA's collection in order to identify the main source of variation for each phytocompound of interest: sugars (sucrose, fructose and glucose), organic acids (citric, malic, succinic and fumaric) and vitamin C (ascorbic acid).

Apricot fruits are a good source of sugars, fiber, proteins, minerals, and vitamins (Moustafa and Cross, 2019). Fruit taste is highly dependent of the soluble solids content, which is the sum of sugars, acids, and other minor components, however sugars represent the most important proportion. As described in apricot and other Prunus species, sucrose, glucose, and fructose are the main sugars present in fruits (Bassi and Selli, 1990; Cirilli et al., 2016). For instance, sucrose is the predominant sugar (40-85\%) in peach, followed by fructose and glucose in variable ratios (Cirilli et al., 2016), similarly to our data presented here. According to Bae et al., (2014), the content of glucose and fructose was higher than sucrose and sorbitol during fruit growth, these authors also pointed that sucrose increase as major sugar in apricot and plum at the end of maturity, that is in accordance with our results. Consumer perception of sweetness intensity depends on the overall sugar amount but also the specific profile (Cirilli et al., 2016). For this sweetness estimation, the contribution of each carbohydrate is calculated, based on the fact that fructose and sucrose are sweeter than glucose (Magwaza and Opara, 2015). Although comparisons with other previous works are complicated for this type of traits, our values are similar to the ones obtained by Fan et al., (2017) analyzing northwest Chinese apricots. According to our study, 'SEOP934', 'HG9821' and 'HG9850' could be good candidates as sweetness source. 
Organic acids also have an important role, with sugars, on apricot taste (Xi et al., 2016). All organic acids increase at first and then fall throughout fruit development and ripening process (Xi et al., 2016). In agreement with the previous studies already cited, malic and citric acids were predominant in the apricot genotypes analysed. In terms of taste Dolenc-Šturm et al., (1999) pointed the stronger acidic taste of malic compared with citric acid, and conclude that the optimal ratio between malic and citric acid is near the value of 0.8. Interestingly, some accessions showed the malic:citric ratio around this value, like 'Dama Rosa' and 'HG9821', two accessions from the IVIA's breeding program, and also 'Goldrich'. Interestingly, the PPV resistant 'Dama Rosa' cultivar has been already registered (Badenes et al., 2018). Moreover, cultivars with high content in acids and low in sugars could be more appreciated, particularly those with higher citric acid concentration (Dolenc-Šturm et al., 1999). Additionally, cultivars with high content of organic acids could be also used as source of these compounds, as they can be used to provide acidity and sour flavour as additive in food products. For instance, malic acid is used for elaboration of sweets and fumaric acid is used as acidulant and antioxidant in soft drinks and cake mixes (Moldes et al., 2017). In this sense, several of the selections studied could be useful for the food-industry, like 'GG9310', 'GG979', and 'SEOP934' that appear as good candidates as they showed high contents of total organics acids.

Finally, the ascorbic acid is one of the most important vitamin in fruits (Lee and Kader, 2000), because of its protective activity as antioxidant (Rice-Evans et al., 1997). We found significant differences in ascorbic acid contents between crop years and among genotypes. Our results are in agreement with others studies on apricot varieties (Akin et al., 2008; Gündoğdu et al., 2013), with values ranging from 98.70 to $192.82 \mathrm{mg} / 100 \mathrm{~g}$ DW among varieties and crop year. 'HM964' could be suggested as a promising cultivar for ascorbic acid content improving due to their high content and stable behaviour in the 3 years.

The increasing demand of healthy products has raised the need of using alternative supplements and additives in food and, fruit nutraceutical compounds can be a good choice since they can be extracted from natural sources and can provide extra health benefits (Moldes et al., 2017). Our results suggest that apricot peel is a good source of sugars, vitamins, and organic acids, being an interesting provider of nutraceutical compounds. Our results are in agreement with other authors that pointed the apricot peel as an extraordinary source of nutraceutical compounds and an optimum tissue for studying mechanisms of flavour quality formation in fruit (Voo et al., 2012; Xi et al., 2016). Similar results were found in previous apricot studies (Ruiz et al., 2005) and other fruits species like pear (Li et al., 2014) or peach (Campbell and Padilla-Zakour, 2013). 


\section{I.6. Conclusions}

A set of selections and genitors from the IVIA's apricot breeding collection has been characterized from a nutraceutical point of view and the main sources of variation of the group of genotypes have been identified, which can be considered as a previous step for further breeding. Our results confirmed the diversity among the set of apricot studied regarding to sugars, organic acids, and ascorbic acid content. These results pave the way for future studies in which the mapping of QTLs can be carried out using our segregating populations once the parents have been characterized. For this purpose, a higher number of fruits will be analysed per tree in order to address the genotype $x$ environment interaction analysis. 
CHAPTER II: Sugar content and sugar-related gene expression in apricot fruits (Prunus armeniaca) for quality breeding 


\section{II.1. Abstract}

Apricot is a stone fruit highly appreciated by consumers, mainly due to their fruits are a good source of sugar compounds. However, fruit quality and sugar balance are crucial for consumer acceptance. Among the stone fruits, apricot is an important source of sugar specially as dried fruit in most of the producing countries. In apricot fruits, the major sugars are sucrose, glucose, fructose, and sorbitol. Regarding to sugar metabolism, the main enzymes involved are sucrose-6-phosphate-synthase (SPS), sucrose synthase (SUS), and sorbitol dehydrogenase (SDH). However, genetic control of sugar content and compounds remains unclear and the number of described sugar-related quantitative trait loci (QTLs) is limited. For a better knowledge of the genetic control of the sugar compounds and their relationships, we have studied the genetic expression of a sorbitol dehydrogenase, a fructokinase, three sucrose synthases, and three phosphate sucrose synthases located in sugar-related QTLs of Prunus genus. The objective is to identify the main actors of the sugar network and their potential use on apricot breeding. The content of the main sugar compounds was analyzed, the orthologous genes identified and confirmed by a phylogenetic tree containing also related species. Results revealed high conservation among Prunus persica (peach) and Prunus armeniaca in the studied predicted proteins. All together, these results contribute to a better knowledge of apricot sugars metabolism, genetic control in the apricot species, and the high homology between apricot and peach related to the genes from the sugar network.

\section{Keywords:}

Sucrose; fructose; apricot; QTL; SPS; SUS; SDH; FK1 


\section{II.2. Introduction}

Apricot is a temperate zone fruit highly appreciated by consumers, mainly due to apricot fruits are a good source of nutraceutic compounds. Indeed, fruit quality is crucial for consumer acceptance (Ruiz and Egea, 2008), especially pomological traits such as aroma, juiciness, flesh colour, fruit weight, shape, sweetness, and soluble sugar concentration (Borsani et al., 2009; Naryal et al., 2019), having the sugar content a clear influence in the consumer preference (Fan et al., 2017; Gurrieri et al., 2001). In apricot fruits, the major sugars are sucrose, glucose, fructose, and sorbitol. Moreover, in some Prunus spp, sugar content depends on the fruit developmental stage and is regulated by carbohydrate supply, dilution effect in fruit volume, and metabolic processes (Desnoues et al., 2016). In fact, sucrose is only detected when fruits reach full maturation (Bae et al., 2014). In addition, soluble solid contents are strongly affected by seasonal variability, in contrast to sugar profiles that tend to be constant across environments and genotypes (Bassi et al., 1996; Cirilli et al., 2016).

The main enzymes involved in sucrose metabolism are sucrose-6-phosphate-synthase (SPS), sucrose synthase (SUS), and sorbitol dehydrogenase (SDH), all of them increased their expression at the end of the fruit ripening (Xi et al., 2016; Zhang et al., 2019). Most of the fruit sucrose concentration comes from the action of the SPS. However, SUS can catalyse the reversible reaction of sucrose into fructose and SDH transforms sorbitol into glucose and fructose with also the action of a sorbitol oxygenase (García-Gómez, 2021). On the other hand, the action of fructokinase (FK), a hexokinase, contributes to the glucose and fructose contents as a result of the sucrose and sorbitol metabolism. Moreover, in those genotypes with high ratios of glucose and fructose, the FK capacities are higher at the end of fruit development (Cirilli et al., 2016; Desnoues et al., 2014).

Regarding genetic control, the number of described QTL related to fruit quality in stone fruits is limited (Abbott et al., 2008), particularly in apricot in which sugar genetic control remains unclear. Nevertheless, some QTLs in apricot related to soluble solids content has been described in LG2, LG3, LG4, and LG5 meanwhile others related to fruit colour are in LG3 (García-Gómez et al., 2019; Salazar et al., 2013). Moreover, a high synteny among Prunus spp. has been described (Arús et al., 2010; Campoy et al., 2011). In peach, sugar-related QTLs have been described in LG1, LG6, and LG7, where also was located a candidate gene for glucose synthase (SUS1) (IIla et al., 2011; Ogundiwin et al., 2009). Improving fruit quality is one of the main goals of the apricot breeding program at Instituto Valenciano de Investigaciones Agrarias (IVIA), along with the development of sharka-resistant varieties. However, phenotypic expression of most of these fruit quality traits are quantitative and based on complex metabolic pathways. Consequently, a better knowledge of the genetic control of these traits and the variability among them could improve the efficiency of breeding programs focused on high-quality varieties (Illa et al., 2011). For this reason, we have studied the genetic expression of a sorbitol 
dehydrogenase, a fructokinase, three sucrose synthases, and three phosphate sucrose synthases located in sugar-related QTLs of Prunus genus in 3 well-known apricot varieties, and 8 hybrid accessions of IVIA's apricot breeding program aimed at unravel the main actors of sugar network and their potential use on apricot breeding.

\section{II.3. Materials and Methods}

\section{II.3.1. Plant Material}

A set of 2 well-known cultivars from the Mediterranean Basin ("Canino' and "Mitger'), 1 NorthAmerican ('Goldrich'), and 8 selections from the IVIA's breeding program resistant to PPV that include 2 registered cultivars ('Dama Rosa' and 'Dama Taronja') were analysed (Table 6). Trees are maintained at the IVIA's germplasm collection located in Moncada, Valencia, Spain. Five fruits per tree were harvested at the ripening stage during two growing seasons (2019 and 2020). For each fruit, the peel was separated from the flesh with a peeler. A mix of peel from 5 fruits was frozen with liquid nitrogen and kept at -80 으 until processing.

Table 6. Plant material and sugar content (g/100g DW).

\begin{tabular}{|c|c|c|c|c|c|c|c|c|}
\hline \multirow{2}{*}{ Genotype } & \multirow{2}{*}{ Pedigree } & \multirow{2}{*}{ Origin } & \multicolumn{2}{|c|}{ Harvest date } & \multirow{2}{*}{ Sucrose } & \multirow{2}{*}{ Glucose } & \multirow{2}{*}{ Fructose } & \multirow{2}{*}{ Sorbitol } \\
\hline & & & 2019 & 2020 & & & & \\
\hline 'Canino' & Unknown & Spain & June 11th & June 3rd & $44,891 \pm 5,959 \mathrm{c}$ & $5.932 \pm 0.806 a$ & $2.140 \pm 0.298 a$ & $5.758 \pm 2.737 \mathrm{bcd}$ \\
\hline $\begin{array}{l}\text { 'Dama } \\
\text { Rosa' }\end{array}$ & Goldrich x Ginesta & IVIA & May 5th & May 29th & $27,767 \pm 2,258 \mathrm{ab}$ & $11.175 \pm 2.422 \mathrm{cdef}$ & $4.603 \pm 0.482 \mathrm{cde}$ & $4.059 \pm 1.545 a b c$ \\
\hline $\begin{array}{c}\text { 'Dama } \\
\text { Taronja' }\end{array}$ & Goldrich x Katy & IVIA & May 5th & June 3rd & $19,737 \pm 3,653 a$ & $14.743 \pm 2.395$ ghi & $6.208 \pm 1.167 f$ & $5.430 \pm 1.044 \mathrm{bcd}$ \\
\hline 'GG9310' & Goldrich x Ginesta & IVIA & May 29th & June 1st & $27,860 \pm 1,660 \mathrm{ab}$ & $9.780 \pm 1.414 \mathrm{bcd}$ & $3.914 \pm 0.940 \mathrm{bc}$ & $2.966 \pm 0.817 \mathrm{ab}$ \\
\hline 'GG979' & Goldrich x Ginesta & IVIA & June 17th & June 5th & $26,849 \pm 10,562 a b$ & $13.742 \pm 3.540 \mathrm{fgh}$ & $5.390 \pm 0.498 \mathrm{def}$ & $2.472 \pm 0.559 a$ \\
\hline 'Goldrich' & Sunglo x Perfection & USA & June 19th & June 11th & $21,565 \pm 8,657 a$ & $15.338 \pm 1.385 \mathrm{hi}$ & $5.697 \pm 0.094$ ef & $2.103 \pm 0.568 \mathrm{a}$ \\
\hline 'GP9817' & Goldrich x Palau & IVIA & June 11th & June 3rd & $21,140 \pm 1,251 \mathrm{a}$ & $13.656 \pm 0.653 \mathrm{efgh}$ & $4.823 \pm 0.542 \mathrm{cde}$ & $4.597 \pm 1.225 \mathrm{abcd}$ \\
\hline 'HG9821' & Harcot x Ginesta & IVIA & June 4th & June 1st & $33,294 \pm 1,110 b$ & $10.444 \pm 0.595$ bcde & $6.082 \pm 0.514 f$ & $2.257 \pm 0.572 a$ \\
\hline 'HG9850' & Harcot x Ginesta & IVIA & May 23th & May 20th & $32,600 \pm 0,777 b$ & $8.269 \pm 0.857 \mathrm{abc}$ & $4.440 \pm 0.268 \mathrm{~cd}$ & $9.264 \pm 1.796 \mathrm{e}$ \\
\hline 'HM964' & Harcot x 'Mitger' & IVIA & May 27th & May 20th & $27,927 \pm 3,641 \mathrm{ab}$ & $7.554 \pm 0.935 a b$ & $3.789 \pm 0.840 b c$ & $6.020 \pm 1.634 \mathrm{~cd}$ \\
\hline 'Mitger' & Unknown & Spain & June 6th & May 25th & $23,398 \pm 4,539 a$ & $17.243 \pm 0.678 \mathrm{i}$ & $6.402 \pm 0.469 f$ & $12.853 \pm 1.420 f$ \\
\hline 'SEOP934' & SEO $\times$ Palau & IVIA & June 3rd & May 25th & $25,715 \pm 2,130 \mathrm{ab}$ & $11,766 \pm 2,448 \mathrm{defg}$ & $3,244 \pm 0,325 b$ & $7,312 \pm 2,475 \mathrm{de}$ \\
\hline
\end{tabular}




\section{II.3.2. Sample processing and HPLC analysis}

Peel samples were freeze-dried and powdered. Tissue homogenization was carried out using a vortex. For sample processing, 10-20 mg of tissue were mixed with $1 \mathrm{~mL}$ of water of LC-MS grade for sugars extraction, homogenized and centrifuged at $4{ }^{\circ} \mathrm{C}$ for $20 \mathrm{~min}$ at $8.050 \mathrm{xg}$. The supernatant was filtered through a $0.45 \mathrm{~mm}$ nylon filter and analysed by HPLC with $20 \mu \mathrm{l}$ of volume injection. The mobile phase consists of $100 \%$ Milli-Q water with a flow rate of $0.8 \mathrm{ml} \mathrm{min}-1$. For retention time determination and sugar quantification, a previous calibration of sucrose, glucose, fructose, and sorbitol was carried out with Sigma Aldrich Standards. HPLC column was kept at $80^{\circ} \mathrm{C}$ in a thermostatically-controlled oven, meanwhile autosampler was at $40 \circ$ C. Each sample was run by quadrupled.

\section{II.3.3. Selection of apricot genes related to sugar content}

A set of 8 key genes previously known as related to sugar content in fruit were analysed in this work (Table 7). Arabidopsis thaliana protein sequences were obtained using a keyword-based searching against the UniProt database. These sequences were used to identify peach and apricot orthologs using the Reciprocal best BLASTP hit method using A. thaliana TAIR10 in Phytozome v.12.1.6 (https://phytozome.jgi.doe.gov/pz/portal.html) and P. persica v.2.1 (Verde et al., 2017) and P. armeniaca v1.0 genomes (Jiang et al., 2019) in the GDR (Jung et al., 2019) websites, respectively. Genomic position of the peach genes were compared with a set of QTLs for sugar content in peach according to previous studies (Cirilli et al., 2016; Desnoues et al., 2016; Sosinski et al., 1998).

\section{II.3.4. Phylogenetic analysis of sugar related genes}

BLASTP analyses were conducted to identify the homologous genes in other species such as Fragaria vesca v1.1 (Shulaev et al., 2011), Vitis vinifera Genoscope.12X (Jaillon et al., 2007) and Malus domestica v.1.0 (Velasco et al., 2010) using Phytozome v.12.1.6 and also Prunus dulcis Texas v.2.0 (Alioto et al., 2020) using the GDR website. Multiple protein sequence alignments were carried out using ClustalW software integrated in MEGA X v.10.1.8 software (Kumar et al., 2018). The proportion of different amino acids between two sequences, $\mathrm{p}$-distance, was calculated for distance estimation. NeighbourJoining phylogenetic trees were built also using MEGA X v.10.1.8 software, and bootstrap supports were calculated using 1000 replicates. 
Table 7. Studied genes and synteny between Arabidopsis thaliana and Prunus persica and Prunus armeniaca.

Prunus persica genome

\begin{tabular}{|c|c|c|c|c|c|c|c|c|c|c|}
\hline \multicolumn{3}{|c|}{ Arabidopsis thaliana } & \multicolumn{2}{|c|}{ Prunus persica } & \multicolumn{4}{|c|}{ Prunus persica vs Arabidopsis thaliana } & \multicolumn{2}{|c|}{$\begin{array}{c}\text { Arabidopsis thaliana vs Prunus } \\
\text { persica }\end{array}$} \\
\hline Gene & TAIR ID & Location & Gene & GDR ID & $\begin{array}{l}\text { Identity with } \\
\text { Arabidopsis CDS }\end{array}$ & E-value & $\begin{array}{l}\text { Identity with } \\
\text { Arabidopsis } \\
\text { Protein }\end{array}$ & E-value & $\begin{array}{l}\text { Identity with } \\
\text { peach Protein }\end{array}$ & E-value \\
\hline AtSDH & AT5G51970.1 & chr5:21111445-21113403 & PperSDH & Prupe.8G143000.1 & $786 / 1050(75 \%)$ & 0 & $274 / 350(78 \%)$ & 0 & $274 / 350(78.29 \%)$ & 0 \\
\hline AtFK1 & AT5G51830.1 & $\operatorname{chr5:21069110-21071739}$ & PperFK1 & Prupe.2G151100.1 & $662 / 918(72 \%)$ & $8 \cdot 10^{-138}$ & $243 / 325(75 \%)$ & $4 \cdot 10^{-173}$ & $243 / 325(74.77 \%)$ & $5.76 \cdot 10^{-150}$ \\
\hline AtSUS1 & AT5G20830.2 & chr5:7050599-7055398 & PperSUS1 & Prupe.7G192300.1 & $1816 / 2408(75 \%)$ & 0 & $659 / 805(82 \%)$ & 0 & $659 / 805(81.86 \%)$ & 0 \\
\hline AtSUS3 & AT4G02280.1 & chr4:994726-998991 & PperSUS3 & Prupe.8G264300.1 & $1859 / 2423(77 \%)$ & 0 & $684 / 810(84 \%)$ & 0 & $684 / 810(84.44 \%)$ & 0 \\
\hline Atsus6 & AT1G73370.1 & chr1:27584364-27588978 & PperSUS6 & Prupe.5G241700.1 & $1735 / 2374(73 \%)$ & 0 & $605 / 811(75 \%)$ & 0 & 605/811 (74.6\%) & 0 \\
\hline AtSPS1 & AT5G20280.1 & chr5:6844712-6850237 & PperSPS1 & Prupe.7G249900.1 & 2323/3039 (76\%) & 0 & $827 / 1058$ (78\%) & 0 & $827 / 1058$ (78.17\%) & 0 \\
\hline AtSPS2 & AT5G11110.1 & chr5:3536189-3541149 & PperSPS2 & Prupe.1G483200.1 & 2234/3065 (73\%) & 0 & 764/1067 (72\%) & 0 & 764/1067 (71.6\%) & 0 \\
\hline AtSPS3 & AT1G04920.1 & chr1:1391434-1395953 & PperSPS3 & Prupe.1G159700.1 & 2302/3169 (73\%) & 0 & $829 / 1076(77 \%)$ & 0 & 836/1077 (77.62\%) & 0 \\
\hline
\end{tabular}

Prunus armeniaca genome

\begin{tabular}{|c|c|c|c|c|c|c|c|c|c|c|}
\hline \multicolumn{3}{|c|}{ Arabidopsis thaliana } & \multicolumn{2}{|c|}{ Prunus armeniaca } & \multicolumn{4}{|c|}{ Prunus armeniaca vs Arabidopsis thaliana } & \multicolumn{2}{|c|}{$\begin{array}{c}\text { Arabidopsis thaliana vs } \\
\text { Prunus armeniaca }\end{array}$} \\
\hline Gene & TAIR ID & Location & Gene & GDR ID & $\begin{array}{c}\text { Identity with } \\
\text { Arabidopsis CDS }\end{array}$ & E-value & $\begin{array}{c}\text { Identity with } \\
\text { Arabidopsis } \\
\text { protein }\end{array}$ & E-value & $\begin{array}{l}\text { Identity with } \\
\text { apricot Protein }\end{array}$ & E-value \\
\hline AtSDH & AT5G51970.1 & chr5:21111445-21113403 & ParSDH & PARG21073m09 & $784 / 1050(75 \%)$ & 0 & $274 / 350(78 \%)$ & 0 & $282 / 371(76.01 \%)$ & 0 \\
\hline AtFK1 & AT5G51830.1 & chr5:21069110-21071739 & ParFK1 & PARG18141m01 & $660 / 918(72 \%)$ & $7 \cdot 10^{-135}$ & $242 / 325(74 \%)$ & $2 \cdot 10^{-170}$ & $244 / 340(71.76 \%)$ & $3.13 \cdot 10^{-145}$ \\
\hline AtSUS1 & AT5G20830.2 & chr5:7050599-7055398 & ParSUS1 & PARG27579m02 & $1821 / 2406(76 \%)$ & 0.0 & $657 / 805(82 \%)$ & 0 & $657 / 805(81.61 \%)$ & 0 \\
\hline Atsus3 & AT4G02280.1 & chr4:994726-998991 & ParSUS3 & PARG19843m01 & $1842 / 2411(76 \%)$ & 0.0 & $677 / 810(84 \%)$ & 0 & $677 / 810(83.58 \%)$ & 0 \\
\hline Atsus6 & AT1G73370.1 & chr1:27584364-27588978 & ParSUS6 & PARG25311m01 & $1734 / 2374(73 \%)$ & 0.0 & $608 / 811(75 \%)$ & 0 & $608 / 811$ (74.97\%) & 0 \\
\hline AtSPS1 & AT5G20280.1 & chr5:6844712-6850237 & ParSPS1 & PARG28120m01 & 2331/3043 (77\%) & 0.0 & $825 / 1058(78 \%)$ & 0 & $825 / 1058(77.98 \%)$ & 0 \\
\hline AtSPS2 & AT5G11110.1 & chr5:3536189-3541149 & ParSPS2 & PARG08259m01 & $1663 / 2163(77 \%)$ & 0.0 & $763 / 1067(72 \%)$ & 0 & 764/1067 (71.6\%) & 0 \\
\hline AtSPS3 & AT1G04920.1 & chr1:1391434-1395953 & ParSPS3 & PARG04316m01 & 2149/2976 (72\%) & 0.0 & 751/978 (77\%) & 0 & 756/980 (77.14\%) & 0 \\
\hline
\end{tabular}




\section{II.3.5. Gene expression}

Total RNA was isolated from $80 \mathrm{mg}$ of powdered tissue with the Plant/Fungi Total RNA Purification Kit (NORGEN, Thorold, ON, Canada), adding 2\% (w:v) polyvinylpyrrolidone (PVP-40) and 2\% Bmercaptoethanol to $600 \mu \mathrm{L}$ of lysis buffer $\mathrm{C}$ for each sample. DNase treatment was made using the RNase-Free DNase Set (Qiagen, Valencia, CA, USA). RNA quality and integrity were checked by agarose gel electrophoresis and quantification was made using the Qubit assay (Invitrogen, Carlsbad, CA, USA). For cDNA synthesis, 500ng of RNA were reverse transcribed with the PrimeScript RT Reagent kit ('Perfect Real Time') using an Oligo-d(T) primer (Takara Bio, Otsu, Japan) in a total volume of $10 \mu \mathrm{L}$. One microliter of 10X diluted first-strand CDNA were used for PCR reactions in a final volume of $15 \mu$. qRT-PCR was performed on a StepOnePlus Real-Time PCR System (Life Technologies, Carlsbad, CA, USA), using TB Green Premix Ex Taq (Tli RNaseH Plus) (Takara Bio, Otsu, Japan). Primer pairs are listed in Table S3. Cycling protocol consisted of $10 \mathrm{~min}$ at $95{ }^{\circ} \mathrm{C}$, followed by 40 cycles of $5 \mathrm{~s}$ at $95{ }^{\circ} \mathrm{C}$ for denaturation and $30 \mathrm{~s}$ at $60^{\circ} \mathrm{C}$ for annealing and extension. PCR reaction specificity was assessed by the presence of a single peak in the dissociation curve after amplification and through size estimation of the amplified products by agarose electrophoresis. Normalized gene expression levels were measured by the relative standard curve procedure using the geometric mean of two reference genes, Actin and Sand-like (Lloret et al., 2017; You et al., 2021). Results were the average of 3 technical replicates each one. Comparisons of multiple samples were evaluated by the non-parametric KruskalWallis test, with a confidence level of 95\%, using the Statgraphics Centurion XVII v. 17.2.00 software (Statpoint Technologies, Warrenton, VA, USA). Significant differences were labelled with different letters.

\section{II.4. Results}

\section{II.4.1. Apricot sugar content}

The sugars profiles showed significant differences $(p \leq 0.05)$ between the accessions analysed (Figure 8; Table S4). Also, Kruskal-Wallis test revealed sucrose and glucose were year-dependant. Regarding total sugar content, "Mitger' (59.9g/100g DW) and 'Canino' (58.7g/100g DW) showed the highest values, while "GP9817' (44.2g/100g DW) and 'Goldrich' (44.7g/100g DW) the lowest ones. In all cases, sucrose was the predominant sugar (ranging from 76.4 to $39.1 \%$ of the total) followed by glucose (34.310.1\%). The third position varied among samples, with fructose ranging from $13.5-3.6 \%$ and sorbitol between 21.5-4.3\%. "Mitger' was the cultivar showing the highest content of both sugars. 


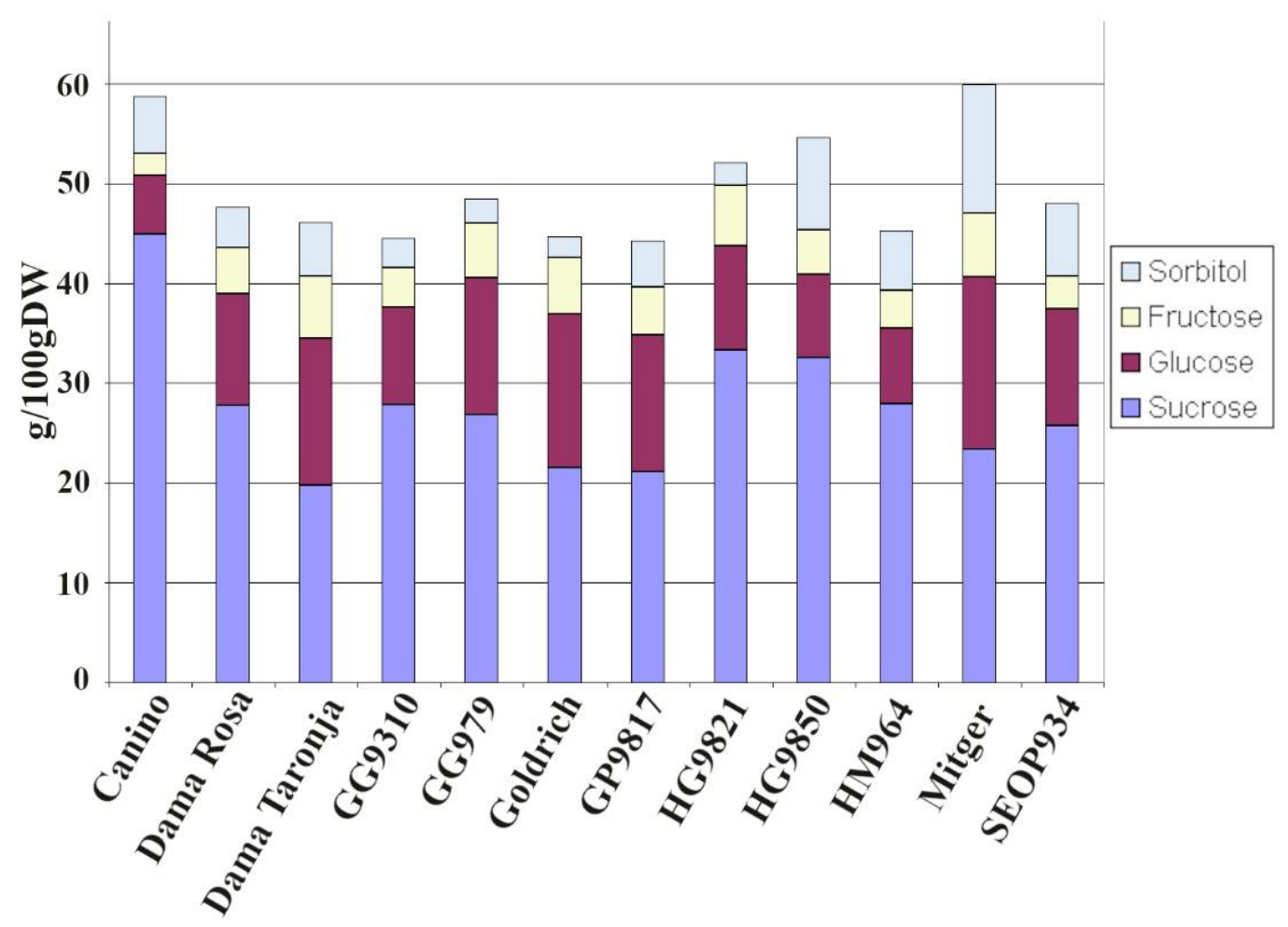

Figure 8. Sugar content profile in the studied accessions (g/100g DW).

\section{II.4.2. Sugar QTL-related genes selection and phylogenetic analysis}

A set of 8 main enzymes genes encoding for 3 sucrose synthases (SUS1, SUS3 and SUS6), a fructokinase (FK1), 3 sucrose-6-phosphate synthases (SPS1, SPS2, and SPS3) and a sorbitol dehydrogenase (SDH) were selected as related with sugar metabolism (Cirilli et al., 2016). Their Arabidopsis thaliana protein and CDS sequences, obtained from UniProt database, were blasted against Prunus persica and Prunus armeniaca predicted proteins in order to identify their homologs (Table 7). As expected, higher similarity was observed between proteins of Arabidopsis and both Prunus spp. (72-84\%), than coding sequences (72-76\%). Location of the Prunus persica proteins in relation with previously described sugar-related QTLs (Table 8) was studied in order to confirm the gene selection for subsequent analysis. Peach genes selected were next to QTLs related to a fruit quality trait, such as soluble sugar content (SSC), fructose, glucose, sucrose content or ripening time. 
Table 8. Peach genes and sugar-related QTLs in Prunus persica.

\begin{tabular}{|c|c|c|c|c|c|c|c|c|}
\hline Name & Gene ID & Gene location & $\mathbf{L}$ & Trait & QTL name & QTL location $^{1}$ & Map name & References \\
\hline \multirow{4}{*}{ PpeSDH } & \multirow{4}{*}{ Prupe.8G143000.1 } & \multirow{4}{*}{$\begin{array}{l}\text { Pp08:15999040- } \\
16001622+\end{array}$} & \multirow{4}{*}{8} & Ripening & qRP.ScB-G8 & - & Peach-LN-F2 (marker) & \multirow{4}{*}{$\begin{array}{l}\text { Desnoues et al., 2016; Sosinski et al., } \\
1998\end{array}$} \\
\hline & & & & Sucrose & qSUC.SZ-LG8.56 & - & Peach-DvsS-BC2 & \\
\hline & & & & Fructose & qFRU.ScB-LG8 & - & Peach-LN-F2; Peach-ScB-F2-1998 & \\
\hline & & & & Glucose & qGLU.ScB-LG8 & - & Peach-ScB-F2-1998 & \\
\hline \multirow{5}{*}{ PpeFK1 } & \multirow{5}{*}{ Prupe.2G151100.1 } & \multirow{5}{*}{$\begin{array}{l}\text { Pp02: 20597751- } \\
\text { 20600680+ }\end{array}$} & \multirow{5}{*}{2} & Fructose & qFRUC.SP-G2.1 & - & Peach-SP-BC2-2004 & \multirow{5}{*}{$\begin{array}{l}\text { Hernández-Mora et al., 2017; Joobeur } \\
\text { et al., 1998; Quilot et al., 2004; } \\
\text { Zeballos et al., } 2016\end{array}$} \\
\hline & & & & SSC & qSSC.peachV2.0-LG2.2017 & $19233501-20101839$ & Prunus-18-peachV2.0-physical & \\
\hline & & & & Ripening & qRT.PF-G2 & - & Prunus-TE-F2 & \\
\hline & & & & time & qRPT.peachV2.0-LG2.201 & $23368508-24174472$ & Prunus-18-peachV2.0-physical & \\
\hline & & & & Total & qTSU.V-Ch2_2-2009 & - & peach-VxBT-F1-Venus & \\
\hline \multirow{3}{*}{ PpeSUS1 } & \multirow{3}{*}{ Prupe.7G192300.1 } & \multirow{3}{*}{$\begin{array}{c}\text { Pp07:18350336- } \\
\text { 18356360+ }\end{array}$} & \multirow{3}{*}{7} & Sucrose & qSUC.SZ-LG7.2_S & $30-39.5$ & Peach-DvsS-BC2 & \multirow{3}{*}{$\begin{array}{l}\text { Desnoues et al., 2016; Quilot et al., } \\
\text { 2004; Romeu et al., 2014; Sosinski et } \\
\text { al., 2000; Vilanova et al., 2003; } \\
\text { Yamamoto et al., } 2005\end{array}$} \\
\hline & & & & & qSUC.SP-G7.1 & - & Peach-SP-BC2-2004 & \\
\hline & & & & $\begin{array}{l}\text { Ripening } \\
\text { time }\end{array}$ & qHD.V6xG-AA-2012.7a & $0-17.8$ & Peach-V6xG-F1-V6 & \\
\hline \multirow{3}{*}{ PpeSUS3 } & \multirow{3}{*}{ Prupe.8G264300.1 } & \multirow{3}{*}{$\begin{array}{l}\text { Pp08:22179197- } \\
\text { 22184773- }\end{array}$} & \multirow{3}{*}{8} & Sucrose & qSUC.SZ-LG8.56 & - & Peach-DvsS-BC2 & \multirow{3}{*}{$\begin{array}{l}\text { Desnoues et al., 2016; Sosinski et al., } \\
1998\end{array}$} \\
\hline & & & & Fructose & qFRU.ScB-LG8 & - & Peach-LN-F2 ;Peach-ScB-F2-1998 & \\
\hline & & & & Glucose & qGLU.ScB-LG8 & - & Peach-ScB-F2-1998 & \\
\hline \multirow{5}{*}{ PpeSUS6 } & \multirow{5}{*}{ Prupe.5G241700.1 } & \multirow{5}{*}{$\begin{array}{l}\text { Pp05:18195911- } \\
18200676+\end{array}$} & \multirow{5}{*}{5} & Sucrose & qSUC.BT-Ch5-2009 & & peac & \multirow{5}{*}{$\begin{array}{l}\text { Desnoues et al., 2016; Hernández Mora } \\
\text { et al., 2017; Zeballos et al., } 2016\end{array}$} \\
\hline & & & & Sugar & qTSU.BT-Ch5-2009 & & & \\
\hline & & & & SSC & qSSC.peachV2.0- & $15249345-18236498$ & Prunus-18-peachV2 0-phvsic & \\
\hline & & & & Ripening & qRPT.peachV2.0-LG5.2017 & $1376476-2240658$ & 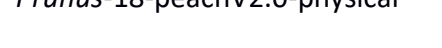 & \\
\hline & & & & FK & qFKACT.SZ-LG5.1_S & $17.2-24.5$ & Peach-DvsS-BC2 & \\
\hline \multirow{4}{*}{ PpeSPS1 } & \multirow{4}{*}{ Prupe.7G249900.1 } & \multirow{4}{*}{$\begin{array}{l}\text { Pp07:21151882- } \\
\text { 21157785- }\end{array}$} & \multirow{4}{*}{7} & & qSUC.SZ-LG7.2_S & $30-39.5$ & Peach-DvsS-BC2 & \\
\hline & & & & & qSUC.SP-G7.1 & - & Peach-SP-BC2-2004 & Desnoues et al., 2016; Quilot et al., \\
\hline & & & & Glucose & qGLC.SZ-LG7.3_S & $32-39.5$ & Peach-DvsS-BC2 & 2004; Romeu et al., 2014 \\
\hline & & & & Ripening & qHD.V6xG-AA-2012.7a & $0-17.8$ & Peach-V6xG-F1-V6 & \\
\hline & & & & & qSUC.SZ-LG1.5_S & 21.1-32.6 & Peach-DvsS-BC2 & \\
\hline PneSPS2 & Prune 1G4832001 1 & Pp01:40288494- & 1 & & qSUC.SZ-LG1.6_S & $25.4-33-4$ & Peach-DvsS-BC2 & Desnoues et al., 2016; Hernández \\
\hline 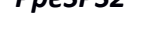 & rifupe. 10403200.1 & 40295210- & $\perp$ & Ripening & qRPT.peachV2.0-LG1.2017 & $40030681-41980791$ & Prunus-18-peachV2.0-physical & Mora et al., 2017 \\
\hline & & & & Fructose & qFRU.SZ-LG1.1_S & 29.1-32 & Peach-DvsS-BC2 & \\
\hline & & & & Sucrose & qSUC.SZ-LG1.6_S & 25.4-33.4 & Peach-DvsS-BC2 & Desnoues et al., 2016; Zeballos et al., \\
\hline PpeSPS3 & Prupe.1G159700.1 & $\begin{array}{l}\text { PpU1:12/0214/- } \\
\text { 12709381- }\end{array}$ & 1 & Fructose & qFRU.BT-Ch1_2-2008 & 23.04 & peach-VxBT-F1-Big_Top & 2016 \\
\hline & & & & Glucose & qGLC.SZ-LG1.1_S & $17.2-33$ & Peach-DvsS-BC2 & \\
\hline
\end{tabular}

${ }^{1}$ Peak or Span start-Span stop. 
Search for homologous proteins were also done in other species in which sugar content in fruit became an important trait, like almond, wild strawberry, grape, and apple (Table S5). As expected, similarity was higher between sequences of Prunus spp. Interestingly, BLASTP analysis of SUS3 and SUS1 from peach or apricot against Fragaria vesca identified the same gene, named mrna12940.1-v1.0-hybrid. Neighbor-joining phylogenetic trees were obtained to study the relationship between species for each gene (Figure 9). In general, almond and peach genes appeared closer to each other than to apricot. Regarding sucrose synthases predicted proteins (Figure 9C), three different clusters can be observed for each gene, but SUS1 and SUS3 clustered together and separated from SUS6, except for MdSUS3. Fragaria gene mrna12940.1-v1.0-hybrid clustered with the rest of SUS1 sequences, hence it was named as FVSUS1. The sucrose-6-phosphate-synthase phylogenetic tree revealed three different clusters (Figure 9D). SPS1 and SPS2 are closer than SPS3. MdSPS1 appears closer to the rest of the sequences of SPS3.

A

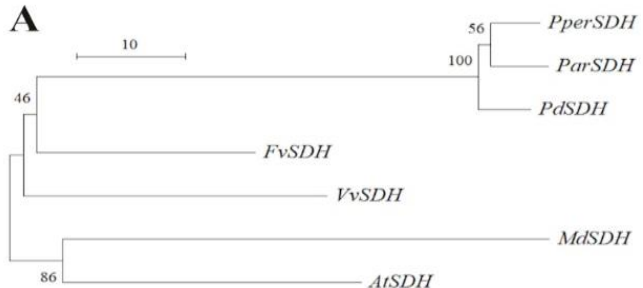

C

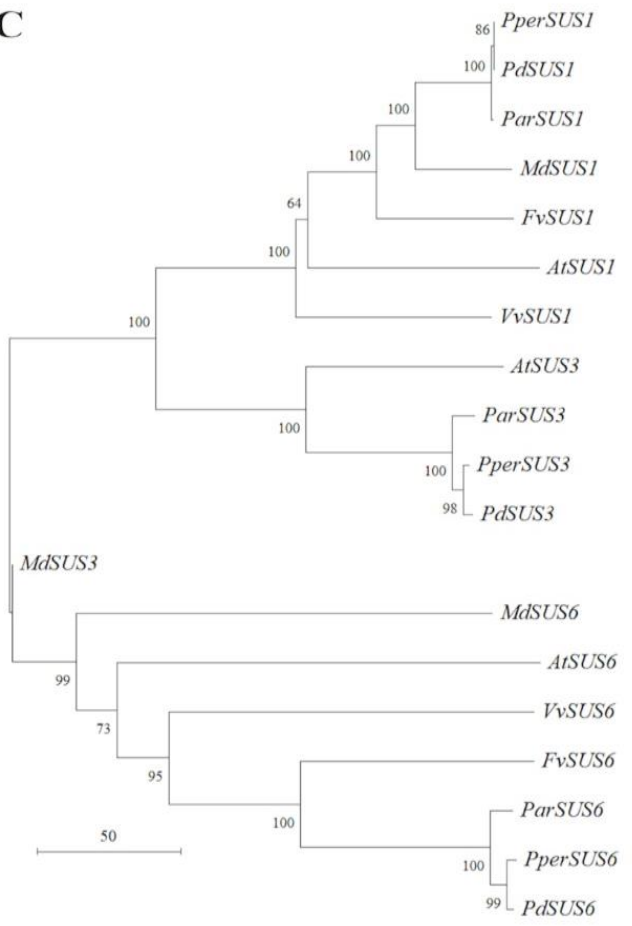

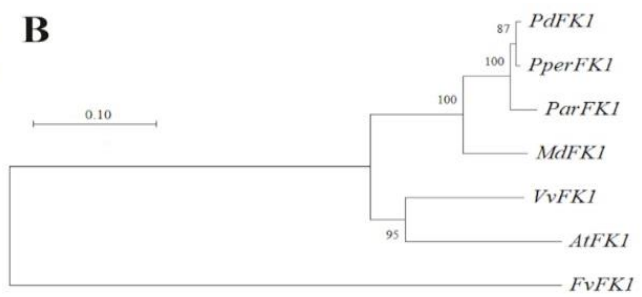

D

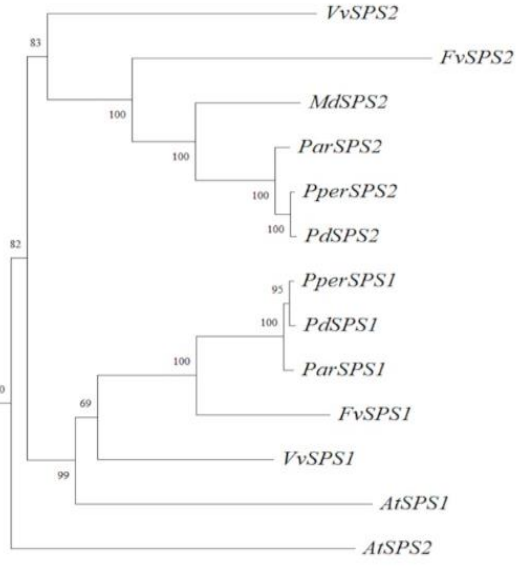

MdSPSI

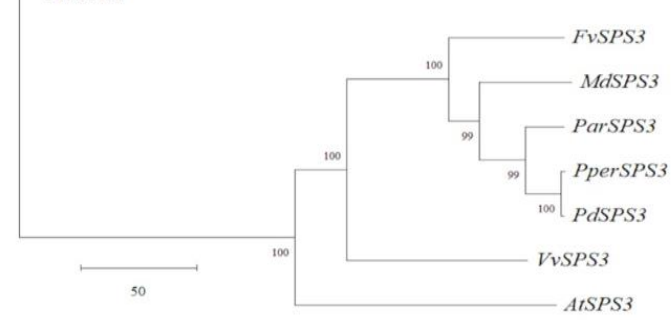

Figure 9. Neighbor-joining phylogenetic trees. A: SDH proteins; B: FK1 proteins; C: SUS proteins; D: SPS proteins. The percentage of replicate trees in which the associated taxa clustered together in the bootstrap test (1000 replicates) are shown next to the branches. 


\section{II.4.3. Gene expression}

Gene expression of the 8 apricot genes was conducted using fruit peel tissue from 11 accessions, 2 well-known cultivars from the Mediterranean Basin, 1 North-American, and 8 selections from the IVIA's breeding program resistant to PPV. The sorbitol dehydrogenase (ParSDH), the fructokinase (ParFK1), the three sucrose synthases (ParSUS1, ParSUS3 and ParSUS6), and three sucrose-phosphatesynthases (ParSPS1, ParSPS2 and ParSPS3) were analysed (Figure 10; Table S6). Statistical analysis revealed ParSPS1, ParSPS3, and ParSDH gene expression as year-dependant.
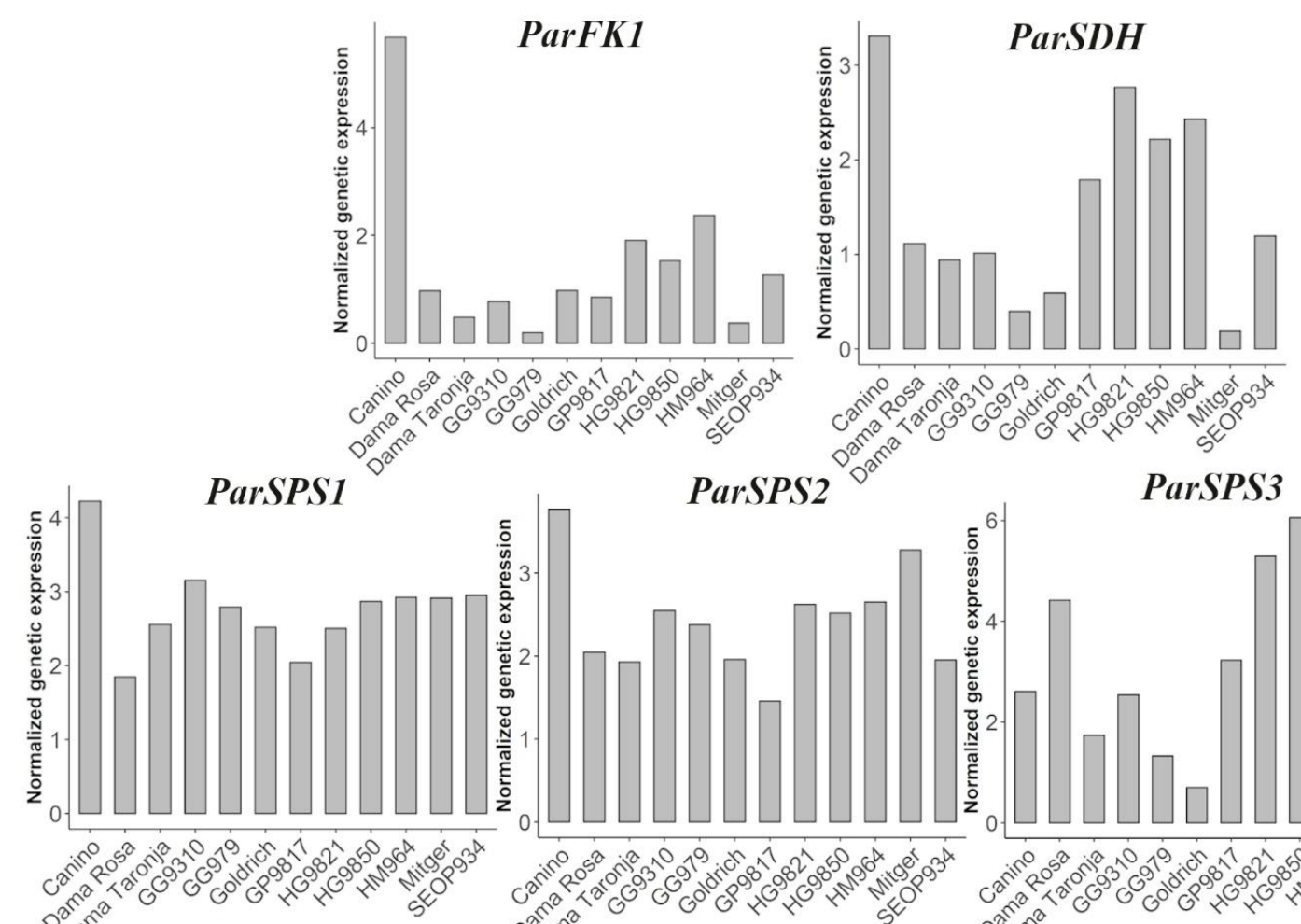

ParSPS2

ParSPS3

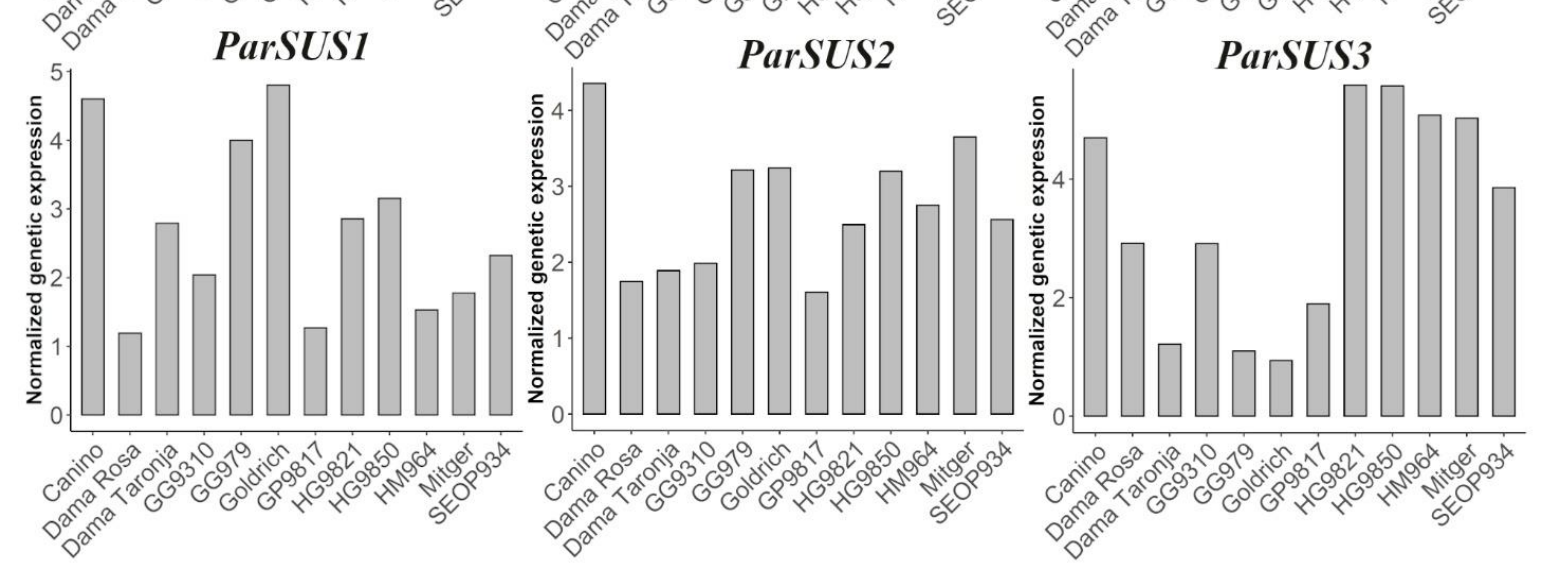

Figure 10. Gene expression of studied apricot genes. 
Fructokinase ParFK1 showed a quite low expression in general, mainly during 2019, but 'Canino' and 'HM964' showed high values in 2020 samples. The sorbitol dehydrogenase ParSDH also showed a very low overall expression and no significant differences among genotypes were observed. Regarding the 3 sucrose-phosphate-synthases (ParSPS1, ParSPS2 and ParSPS3), 'Canino' was the accession with the highest expression for ParSPS1 and ParSPS2 for both years, but not for SPS3. For this gene, the highest expression level in 2019 was observed in 'HG9821', but in 'HG9850' in 2020 and also for the two yearsaverage. In reference to sucrose synthase, two-years average results revealed 'Goldrich' and 'Canino' as the accessions with the highest expression for ParSUS1 and ParSUS3, respectively. However, 'Canino' also revealed a high expression in ParSUS1. Regarding ParSUS6, no significant differences among genotypes were found in 2019 samples but more differences were observed in 2020, with lower expression in samples like 'Goldrich', 'GG979' or 'Dama Taronja'.

\section{II.5. Discussion}

Apricots can be an important source of different nutraceutical compounds such as fibbers, vitamins, phenolics or soluble solids. In addition, some of them are in higher amounts in the peel than in flesh, as occurs in other fruit crops (Bizjak et al., 2013; Gómez-Martínez et al., 2021). For this reason, improvement of fruit quality parameters could be a crucial aspect to develop new well-appreciated varieties. In this sense, it should be taken into account that an excellent balance between sugars and organic acids is relevant for consumer appreciation and consumption (Xi et al., 2016). However, sugar genetic control in apricot is still unclear. Advancing knowledge about the genetic control of these traits could improve the efficiency of apricot breeding programs. In this work, we analyse the sugar profile in fruit peel of 11 accessions, 2 well-known cultivars from the Mediterranean Basin, 1 North-American, and 8 selections from the IVIA's breeding program. Our results showed that at maturity, sucrose was the major sugar in the apricot peel, followed by glucose. In contrast, fructose and sorbitol were the minor studied sugar compounds. These results are in agreement with previous studies in apricot and peach (Bae et al., 2014; Dirlewanger et al., 1999; Wang et al., 2016; Xi et al., 2016).

Among the studied genotypes, 'Canino' was the accession with the highest concentration in sucrose, which agrees with the high expression observed for sucrose synthase (ParSUS1 and ParSUS3) and sucrose-phosphate-synthases (ParSPS1 and ParSPS2). On the other hand, some studies suggested that the metabolic capacity of sorbitol dehydrogenase in peach could be responsible of fructose levels in fruits (Kanayama et al., 2005). This fact could be related with the low concentration of fructose and sorbitol in all studied accession, and the low expression level for ParSDH and ParFK1 showed in all genotypes during the period of study. However, significant differences among genotypes in the contain of soluble sugar compounds were found, but not at their gene expression levels in all cases. Similar 
results have been observed in other studies about sugar-related enzymes in peach fruits, in which a slight variation in enzymatic activity was insufficient to explain changes in metabolites concentrations, revealing stability across years and genotypes with variable sugar composition (Cirilli et al., 2016). In addition, sugar accumulation is a quantitative trait affected by environment conditions, different interconnected metabolic processes or multigenic-control (Cirilli et al., 2016) and cannot be explained only by gene expression.

Concerning genetic control knowledge, a total of six SUS genes are present in the Prunus persica genome (Cirilli et al., 2016). From them, PperSUS1 is prevalently expressed in peach fruit compared with others plant parts, being also upregulated during fruit ripening and postharvest storage (Zhang et al., 2013). A similar situation is presented for SPS genes. Although four putative genes are identified in peach, only SPS1 and SPS2 have been studied in detail (Cirilli et al., 2016). In this study, we analyse the expression in the apricot peel of SUS1, SUS3 and SUS6 and SPS1, SPS2 and SPS3 of peach orthologues in a set of 11 apricot genotypes during two growing seasons which extends the number of genes studied in Prunus genus. As a first step to identify orthologues in different species, a BLAST of Arabidopsis thaliana against Prunus persica and Prunus armeniaca genome references were performed and showed high similarity among predicted protein sequences, particularly between peach and apricot. In addition, a search of orthologues in other economically important species was carried out. In agreement with other studies (Cirilli et al., 2016; Socquet-Juglard et al., 2013), high conservation among Prunus spp. and another Rosaceae species was observed (Arús et al., 2010).

Their position related to previously described QTLs associated with a sugar-related trait, such as soluble solids, sucrose, fructose, and glucose concentration was studied. However, a few studies in apricot showed a relationship between fruit ripening time and sugar profiles (Mesarović et al., 2018). As far as sugar concentration is extremely related to ripening time, QTLs related to this trait were also analysed for gene locations. The QTL screening in apricot just revealed an SSC-related QTL located in LG2, and only a few related with flowering or ripening time located near the studied genes (Hernández Mora et al., 2017; Joobeur et al., 1998; Quilot et al., 2004; Zeballos et al., 2016). Peach genome is more complete and has a high synteny among Rosaceae spp., particularly this synteny is very high between Prunus armeniaca and Prunus persica (Jiang et al., 2019). Results showed peach orthologues for all sucrose-phosphate and sucrose synthases analysed were located near a described sucrose QTL, and all of them were located near a fructose-related QTL. In fact, PperFK1, located in LG2, was related with a fructose-related QTL but also with fruit ripening time and SSC traits. These results are in agreement with other studies in peach that support a correlation between ripening date and sugar content by a QTL co-localization on LG2 and by the co-localization of a QTL for SCC at the MD locus, establishing a possible pleiotropic effect among them (Dirlewanger et al., 2006). 
On the other hand, other studies in Prunus (Desnoues et al., 2016) identified different QTLs for sucrose but the most important QTL was located in LG1. This result agrees with our results, as PperSPS2 and PperSPS3 are close to a QTL related with sucrose in LG1.

Although the low detail found in the apricot reference genome about sugar-related QTLs, an association has been established in the localization of orthologs in peach with QTLs associated with the genetic control of sugars. However, due to the high synteny found between both species, it would be expected that a similar situation could also occur in apricot fruits. However, more QTLs needs to be identified with the sugar profile for a better understanding of the genetic control of sugars in apricot. On the other hand, the study of the profile of the sugar content can be interesting for the development of varieties with a balance taste, but the knowledge of the profile in byproducts such as fruit peel can be interesting also for the development of new varieties or the use of existing ones by the cosmetic or food industry.

\section{II.6. Conclusions}

In this work we analysed the sugar profile in apricot peel and also, we studied the gene expression of relevant genes involved in sugar metabolism: sorbitol dehydrogenase (ParSDH), a fructokinase (ParFK1), three sucrose-synthases (ParSUS1, ParSUS2 and ParSUS3), and three sucrose-phosphatesynthases (ParSPS1 and ParSPS2). Results demonstrated a high conservation among Prunus persica and Prunus armeniaca in the studied predicted proteins. Additionally, we showed the relationship among sugar-related QTLs detected in peach and the orthologous obtained. All together, these results contribute to a better knowledge of sugars genetic control in the apricot species and for homology found to Prunus persica as well. 


\section{CHAPTER III: Polyphenol content in apricot fruits}

This section is based on an article published in Scientia Horticulturae (2021, vol. 277, 109828).

The final authenticated version is available online at:

https://doi.org/10.1016/j.scienta.2020.109828

Authors contributions:

- Helena Gómez-Martínez: Data curation, writing original draft, formal analysis, writing review and editing.

- A. Bermejo: Data curation.

- E. Zuriaga: Supervision, writing review and editing.

- M.L. Badenes: Project administration, writing original draft, writing review and editing. 


\section{III.1. Abstract}

Apricot (Prunus armeniaca L.) species is one of the most important Mediterranean fruits. The fruits are important in the diet of Asian and Mediterranean countries in which the apricot is used as fresh and dried fruit, being an important source of nutrients. Despite of the amount of genetic resources and diversity studies available into the species, there are a few studies focused on fruit quality. Among the different compounds of fruit quality, polyphenols are classified as the most abundant antioxidants in nature, being important as a source of health benefits as well as a potential source of natural products for the food industry. The important role of polyphenols in human nutrition, outline these compounds as the most relevant for defining fruit quality. In this study, the polyphenol content on fruits from different apricot varieties included elite cultivars and hybrids from the IVIA breeding program have been compared for identifying the genotypes with relevant contribution to fruit quality. The most important compounds obtained in terms of quantity were: phenolic acids and flavonoids. Results identified the PPV resistant cultivar 'Goldrich' as the best cultivar for increasing the content of antioxidants in the varieties of the breeding program.

\section{Keywords:}

Fruit quality, antioxidants, neochlorogenic, chlorogenic, rutin, Quercetin-3-glucuronide 


\section{III.2. Introduction}

Apricot (Prunus armeniaca L.) species is one of the most important Mediterranean fruits. Its center of origin is located in China and later it spread to Europe and the rest of Asian countries generating different ecological diversification centers in which the Mediterranean basin is one of them (Bailey and Hough, 1975). The long domestication history provided a wide genetic diversity in pomological characteristics and adaptability to different environments. The fruits are important in the diet of Asian countries in which the apricot is used as fresh and dried fruit, being an important source of sugar. Despite the genetic diversity of apricot species has been very well studied (Martínez-Mora et al., 2009; Romero et al., 2003; Z. Wang et al., 2014a) there are few studies focused on compounds related to fruit quality (Camps and Christen, 2009; Socquet-Juglard et al., 2013; Ruiz et al., 2005). Among the different compounds polyphenols are one of the most important as a source of health benefits as well as a potential source of natural products for the food industry. Polyphenols represent a group of chemical substances common in plants being the different parts of the plants the main provider of these important compounds in the human diets. Polyphenols are positively correlated with antioxidant capacity of fruits (Almeida et al., 2011; Gan et al., 2016; Mokrani et al., 2016). Hence, one of the most important benefits of fruit consumption is attributed to their high antioxidant content. Research studies supports the role of antioxidants in the prevention of several diseases (Ginter and Simko, 2012; Manach et al., 2005; Rodriguez-Mateos et al., 2014; Scalbert et al., 2005).

The involvement of reactive oxygen species (ROS) in the etiology of many diseases suggested that phytochemicals showing antioxidant activity may contribute to the prevention of these pathologies. In this sense polyphenols provide health benefits by elimination of free radicals, by the protection and regeneration of other dietary antioxidants (e.g. vitamin E) and the chelation of pro-oxidant metals (Lima et al., 2014). Their antioxidant potential provides other health benefits reported such as an antimutagenic activity, reduction of the risk of cardiovascular diseases, atherosclerosis protection (Yao et al., 2004). Dietary polyphenols contribute to epigenetic changes at cell level and have emerged as potential drugs for therapeutic uses.

In the food industry, preservation of food requires the addition of antioxidant compounds. Some plant extracts may represent an alternative source of natural antioxidants, that can be included in the human diet of being an important source for synthesis of these compounds as natural additives of the food industry. Polyphenol concentrations in foods vary according to numerous genetics and environmental factors (Manach et al., 2004). Differences on polyphenol content among cultivars from different species have been reported, pointing out the genetic diversity (Andre et al., 2007; Tabart et al., 2006). In temperate fruit crops, polyphenol content is relevant and arise as one of the main contributor to fruit quality (Veberic and Stampar, 2005). Polyphenol content and antioxidant activity of fruits have 
been very well referenced (Wolfe et al., 2003). For instance, the role on health benefits of phenolic compounds from apple was studied by Boyer and Liu (2004). The polyphenolic content varied among apple cultivars, remaining relatively stable during cold storage (Matthes and Schmitz-Eiberger, 2009) being an important feature for apple consumption. The studies of polyphenols in stone fruits are scarce and focused on antioxidant capacity, in nectarines and plums (Gil et al., 2002; Kim et al., 2003) and apricot (Erdogan-Orhan and Kartal, 2011; Fan et al., 2018). Besides of the antioxidant capacity, polyphenols fruit content is becoming an important component of fruit quality because affect the color, flavor and taste of the fruits, impacting the fruit consumption (Crisosto, 2003).

Polyphenols have been related to colour of fruits and anthocyanin accumulation (Jin et al., 2016; Luo et al., 2016). Several genes have been identified in the metabolic pathways, such as dihydroflavonol 4reductase (DFR) and flavonol synthase (FLS), associated with anthocyanin pathway. On the other hand, in Prunus genus, MYB10 gene has been proposed as the best candidate for skin colour in peach (Jiao et al., 2014; Rahim et al., 2014; Tuan et al., 2015) and apricot fruit (García-Gómez et al., 2019). In addition, some candidate genes have been reported for skin pigmentation in peach, such as a betacarotene hydroxylase $(B C H)$, a zeaxanthin epoxidase (ZXE2) and a leucoanthocyanidin dioxygenase (PpLDOX) (Ogundiwin et al., 2009 and 2008). All the genes identified in the polyphenols pathways represent new strategies for increasing fruit quality by means of conventional and molecular breeding. The important role of polyphenols in different plant mechanisms as well as their increasing importance in human nutrition, outline these compounds as the most relevant for defining fruit quality. In apricot the outbreak of the sharka diseases caused by the plum pox virus or PPV (García et al., 2014), point out the need of introgression of resistance as the unique solution. Only a few cultivars from the Ontario region of Canada were identified as resistance to PPV (Soriano et al., 2012). Apricot as a temperate fruit crop needs to accomplish an amount of chilling during winter for spring budbreak. The resistant cultivars available have high chilling requirements. This mechanism of adaptability gathered during evolution results in bad adaptability to warmer winters as those of the Mediterranean area. Beside of the bad adaptability, the resistant cultivars provided other inconvenient characteristics as floral selfincompatibility and worse fruit quality. The introgression of resistance to PPV in apricot may have important consequences in the new obtained resistant cultivar as compromised adaptability and worse fruit quality.

Our hypothesis is that among the group of cultivars resistant to PPV, 'Goldrich' is the better adapted to the Mediterranean conditions. This cultivar has been used as the main donor of resistance in the IVIA breeding program (Badenes et al., 2018). In this study, we test the potential effect on fruit quality of the main donor of resistance to PPV and their suitability for increasing fruit quality in the program. Due to the important role of polyphenols in fruit quality we focused the study on these compounds. 
The relationship between phenolic components and the genotypes and structure of the data were analyzed using principal component analysis (PCA).

The study presents and compares the polyphenol content on fruits from different apricot varieties that included the main donor of resistance to PPV, traditional varieties adapted to the Mediterranean and the first generation of hybrids from the IVIA breeding program aimed at identifying the best genitors for increasing the content of antioxidants in the elite varieties.

\section{III.3. Material and methods}

\section{III.3.1. Plant Material}

The plant material consisted in a set of cultivar and selections from the IVIA's breeding program (Badenes et al., 2006; Martínez-Calvo et al., 2009) that aims to obtain new varieties resistant to PPV (plum pox virus) the most important disease affecting Prunus genus species worldwide (García and Cambra, 2007; García et al., 2014). A set of 4 well-known cultivars (group 1) and 9 selections (group 2) from the IVIA's breeding program were analysed (Table 9). First group includes "Canino', "Mitger' and 'Tadeo', all three cultivars from the Mediterranean Basin, and 'Goldrich' a variety from North America, used as a donor of resistance to PPV. Second group includes 2 cultivars already registered 'Dama Rosa' and 'Dama Taronja' and other 7 preselected accessions All of them are selected seedlings resistant to PPV and self-compatible.

Table 9. Plant material.

\begin{tabular}{c|c|c|ccc}
\hline \multirow{2}{*}{ Genotype } & Pedigree & \multirow{2}{*}{ Origin } & \multicolumn{3}{|c}{ Harvest Date } \\
\cline { 4 - 6 } & & & $\mathbf{2 0 1 6}$ & $\mathbf{2 0 1 7}$ & $\mathbf{2 0 1 8}$ \\
\hline 'Canino' & Unknown & Spain & June 3 & May 31 & June 11 \\
'Dama Rosa' & Goldrich x Ginesta & IVIA & June 6 & June 9 & June 7 \\
'Dama & Goldrich x Katy & IVIA & June 10 & June 9 & June 11 \\
'GG9310' & Goldrich x Ginesta & IVIA & June 6 & June 9 & June 5 \\
'GG979' & Goldrich x Ginesta & IVIA & June 13 & June 9 & June 14 \\
Goldrich & Sunglo x Perfection & USA & June 22 & June 9 & June 11 \\
'GP9817' & Goldrich x Palau & IVIA & June 13 & June 9 & June 11 \\
'HG9821' & Harcot X Ginesta & IVIA & June 8 & May 25 & June 5 \\
'HG9850' & Harcot x Ginesta & IVIA & June 3 & May 25 & June 7 \\
'HM964' & Harcot x 'Mitger' & IVIA & June 1 & June 2 & May 30 \\
'Mitger' & Unknown & Spain & June 3 & May 25 & May 30 \\
'SEOP934' & SEO x Palau & IVIA & June 8 & June 2 & June 5 \\
'Tadeo' & Unknown & Spain & June 15 & June 9 & June 18 \\
\hline
\end{tabular}


The trees are maintained at the IVIA's germplasm collection located in Moncada (latitude $37^{\circ} 45^{\prime} 31.5^{\prime \prime}$ N., longitude $1^{\circ} 01^{\prime} 35.1^{\prime \prime}$ W.), near Valencia (Spain). The genotypes were characterized for agronomic and pomology traits for further selection. The pomological characterization of the genotypes studied was made following Martínez-Calvo et al. (2010). Variables related to fruit size and firmness were indicated in Table 10.

Table 10. Pomological traits measured in the genotypes studied related to fruit size and firmness. 3years average \pm standard deviation. Different letter means significant differences among genotypes.

\begin{tabular}{|c|c|c|c|c|c|c|c|}
\hline Genotype & $\begin{array}{l}\text { Height } \\
(\mathrm{mm})\end{array}$ & $\begin{array}{l}\text { Diameter } \\
(\mathrm{mm})\end{array}$ & $\begin{array}{c}\text { Ratio } \\
\frac{\text { Height }}{\text { ventralwidth }}\end{array}$ & $\begin{array}{c}\text { Weight } \\
\text { (g) }\end{array}$ & $\begin{array}{l}\text { Weight } \\
\text { (stone)(g) }\end{array}$ & $\begin{array}{c}\text { Ratio } \\
\frac{\text { weight }(\text { fruit })}{\text { weight }(\text { stone })}\end{array}$ & $\begin{array}{l}\text { Firmness } \\
\left(\mathrm{kgf} / \mathrm{cm}^{2}\right)\end{array}$ \\
\hline 'Canino' & $44.9 \pm 6.9$ def & $45.9 \pm 7.9 b$ & $1.3 \pm 0.3$ ef & $61.4 \pm 21.5 \mathrm{~d}$ & $3.5 \pm 0.4 \mathrm{fg}$ & $17.2 \pm 4.9 \mathrm{abc}$ & $2.8 \pm 1.7$ cde \\
\hline $\begin{array}{l}\text { 'Dama } \\
\text { Rosa' }\end{array}$ & $41.7 \pm 2.1 \mathrm{bcd}$ & $46.5 \pm 2.3 b$ & $1.1 \pm 0.1 \mathrm{bc}$ & $49.3 \pm 6.5 b c$ & $3.2 \pm 0.2 \mathrm{def}$ & $15.7 \pm 2.6 \mathrm{a}$ & $1.5 \pm 0.5 \mathrm{abc}$ \\
\hline $\begin{array}{c}\text { 'Dama } \\
\text { Taronja' }\end{array}$ & $52.5 \pm 5.6 \mathrm{~h}$ & $52.5 \pm 6.4 \mathrm{~d}$ & $1.6 \pm 0.3 \mathrm{~g}$ & $85.5 \pm 25.2 f$ & $5.5 \pm 1.4 \mathrm{~h}$ & $16.2 \pm 6.3 a b$ & $1.5 \pm 1.4 \mathrm{abc}$ \\
\hline 'GG9310' & $43.1 \pm 3.8 \mathrm{cde}$ & $46.8 \pm 4.7 b$ & $1.2 \pm 0.2 \mathrm{cde}$ & $57.8 \pm 13.3 \mathrm{bcd}$ & $2.7 \pm 0.4 \mathrm{bcd}$ & $21.4 \pm 4.0 \mathrm{~d}$ & $0.6 \pm 0.3 \mathrm{a}$ \\
\hline 'GG979' & $46.0 \pm 5.1 \mathrm{efg}$ & $50.8 \pm 6.5$ & $1.4 \pm 0.2 \mathrm{f}$ & $73.4 \pm 18.7$ e & $3.8 \pm 0.7 \mathrm{~g}$ & $19.4 \pm 4.1 \mathrm{bcd}$ & $1.1 \pm 0.6 \mathrm{ab}$ \\
\hline 'Goldrich' & $49.2 \pm 4.0 \mathrm{gh}$ & $46.9 \pm 3.2 b$ & $1.3 \pm 0.1 \mathrm{ef}$ & $60.6 \pm 10.8 \mathrm{~cd}$ & $3.8 \pm 0.5 \mathrm{~g}$ & $16.4 \pm 4.0 \mathrm{abc}$ & $2.2 \pm 1.4 \mathrm{bcde}$ \\
\hline 'GP9817' & $41.9 \pm 3.5 \mathrm{bcd}$ & $48.5 \pm 4.0$ & $1.1 \pm 0.2 \mathrm{bcd}$ & $54.5 \pm 13.1 \mathrm{bcd}$ & $3.2 \pm 0.5$ ef & $17.2 \pm 2.8 \mathrm{abc}$ & $1.5 \pm 1.2 \mathrm{ab}$ \\
\hline 'HG9821' & $47.4 \pm 3.4 \mathrm{fg}$ & $53.4 \pm 4.7 d$ & $1.4 \pm 0.2 \mathrm{fg}$ & $77.1 \pm 12.4$ ef & $3.1 \pm 0.5 \mathrm{cde}$ & $25.7 \pm 5.1 \mathrm{e}$ & $2.9 \pm 3.4 \mathrm{de}$ \\
\hline 'HG9850' & $43.6 \pm 2.9 \mathrm{cde}$ & $47.8 \pm 3.1$ & $1.3 \pm 0.2 \mathrm{de}$ & $60.2 \pm 12.2 \mathrm{~cd}$ & $3.0 \pm 0.5$ bcde & $20.5 \pm 3.8 d$ & $3.1 \pm 2.7 \mathrm{e}$ \\
\hline 'HM964' & $37.5 \pm 4.2 \mathrm{a}$ & $45.4 \pm 4.5 b$ & $1.0 \pm 0.2 b$ & $48.4 \pm 15.7 b$ & $2.6 \pm 0.3 b c$ & $19.1 \pm 5.3 \mathrm{abcd}$ & $1.7 \pm 0.9 \mathrm{abcd}$ \\
\hline 'Mitger' & $42.3 \pm 3.5 \mathrm{~cd}$ & $46.8 \pm 4.7 b$ & $1.1 \pm 0.2 b c$ & $51.7 \pm 15.6 \mathrm{bcd}$ & $2.6 \pm 0.4 b c$ & $19.6 \pm 4.6 \mathrm{~cd}$ & $2.9 \pm 1.3 \mathrm{de}$ \\
\hline 'SEOP934' & $38.9 \pm 3.0 a b$ & $47.2 \pm 1.9$ & $1.1 \pm 0.1 \mathrm{bcd}$ & $52.7 \pm 5.3 \mathrm{bcd}$ & $2.6 \pm 0.3 b$ & $20.5 \pm 2.2 \mathrm{~d}$ & $1.0 \pm 0.6 \mathrm{ab}$ \\
\hline 'Tadeo' & $36.8 \pm 2.9 a$ & $40.1 \pm 3.3 \mathrm{a}$ & $0.8 \pm 0.1 \mathrm{a}$ & $33.0 \pm 8.5 a$ & $1.6 \pm 0.3 a$ & $20.8 \pm 4.0 \mathrm{~d}$ & $3.1 \pm 1.2 \mathrm{e}$ \\
\hline
\end{tabular}

For polyphenols analysis, five fruits per tree were harvested the ripening stage during 3 growing seasons (2016, 2017 and 2018). For each fruit, the peel was separated from the flesh with a peeler. Two samples consisted in a mix of the peel from 5 fruits and a mix of flesh from 5 fruits per genotype and crop year were frozen with liquid nitrogen and kept at $-80 \circ \mathrm{C}$ until processing. Tissue homogenization was carried out using a Polytrom 3100 (Kinematica AG, Switzerland) and a vortex for the flesh and peel samples, respectively.

\section{III.3.2. Extraction and HPLC of phenolic compounds}

Phenolics were extracted and determined according to the procedure described by Cano et al. (2008) and Cano and Bermejo (2011). Briefly, $5 \mathrm{mg}$ of freeze-dried peel or flesh were mixed with $1 \mathrm{~mL}$ of 
DMSO/MeOH $(1: 1, v / v)$. Then the sample was centrifuged (Eppendorf 5810R centrifuge; Eppendorf Iberica, Madrid, Spain) at $4{ }^{\circ} \mathrm{C}$ for $20 \mathrm{~min}$ at $8.050 \times \mathrm{g}$. The supernatant was filtered through a $0.45 \mu \mathrm{m}$ nylon filter and analysed by HPLC-DAD and HPLC-MS in a reverse-phase column C18 Tracer Excel $5 \mu \mathrm{m}$ 120 OSDB (250 mm x $4.6 \mathrm{~mm}$ ) (Teknokroma, Barcelona, Spain). An Alliance liquid chromatographic system (Waters, Barcelona, Spain) equipped with a 2695 separation module, coupled to a 2996 photodiode array detector and a ZQ2000 mass detector was used. A gradient mobile phase consisting of acetonitrile (solvent $A$ ) and $0.6 \%$ acetic acid (solvent $B$ ) was used at a flow rate of $1 \mathrm{~mL} / \mathrm{min}$, with an injection volume of $10 \mu \mathrm{L}$. The gradient change was as follows: $10 \% 2 \mathrm{~min}, 10-75 \% 28 \mathrm{~min}, 75-10 \% 1$ min, and hold at 10\% 5 min. An HPLC-MS analysis was performed and worked under electrospray ion positive (flavonoids) and negative (phenolic acids) conditions. Capillary voltage was $3.50 \mathrm{kV}$, cone voltage was $20 \mathrm{~V}$, source temperature was $100^{\circ} \mathrm{C}$, desolvation temperature was $225^{\circ} \mathrm{C}$, cone gas flow was $70 \mathrm{~L} / \mathrm{h}$ and desolvation gas flow was $500 \mathrm{~L} / \mathrm{h}$. Full data acquisition was performed by scanning 200 to 800 uma in the centroid mode. Compounds were identified on the basis of comparing their retention times, UV-Vis spectra and mass spectrum data with authentic standards from Sigma-Aldrich using an external calibration curve. All the solvents used were of LC-MS grade. Three samples per cultivar were analysed.

\section{III.3.3. Data analysis}

All the data analysis and graphics were made using R-studio software (Version1.1.463, 2009-2018, Rstudio, Inc.) with 'stats', grDevices', and 'graphics' (R Core Team), 'dplyr' (Wickham, et al, 2021), 'readxl' (Wickham, et al., 2016a), 'plyr' (Wickham, 2020), 'scales' (Wickham and Seidel, 2020), 'grid' (Murrell, 2005), 'ggbiplot' (Vu, 2011.), 'FSA' (Ogle et al., 2020), 'DescTools' (Signorell, et al., 2020), 'rcompanion' (Mangiafico, 2020), 'multcompView' (Graves, et al., 2019), and 'ggplot2' (Wickham, 2016b) packages.

Polyphenol content from all compounds and accessions were statistically tested by Kruskal-Wallis test $(P \leq 0.05)$ and averages were compared with the Pairwise Wilcoxon-Mann-Whitney test at $95 \%$ confidence level $(P \leq 0.05)$, using the Statgraphics XVI.I software (Statpoint Technologies, Warrenton, VA, USA). Significant different samples were labeled with different letters. Data of the accessions were analysed by multivariate analysis, applying the method of Principal Components Analysis (PCA) (Eriksson et al., 1999). PCA and correlogram were carried out using R (v.3.6.1, R Core Team, 2019) with R-studio software (v.3.5.3) with the 'stats' (R Core Team), 'ggplot2' (Wickham, 2016b), 'GGally' (Schloerke, et al., 2020), 'dplyr' (Wickham, et al, 2021), and 'factoextra' (Kassambara and Mundt, 2020). Previously, data was centred and scaled to have unit variance. The variables included were the compounds analyzed. A biplot of individual scores and loadings was obtained.

For testing the contribution of 'Goldrich' to the parameters of quality in the studied population, we 
performed a regression of the data to a linear model as described by Gómez and Ligarreto (2012). In the model, the phenotype is linearly explained as follows:

$$
\text { [ Phenotype }=C+G_{\text {Goldrich }}+\text { Year }+G_{\text {Goldrich }} \text { Year }+ \text { Residual ] }
$$

Where $C$ is the general average of the population (constant), $G_{\text {Goldrich }}$ is the genetic effect of 'Goldrich', Year is the environmental effect due to the year and Residual is the residual effect.

The model was calculated using the Statgraphics XVI.I software (Statpoint Technologies, Warrenton, VA, USA). A quantitative variable for evaluating the genetic effect of 'Goldrich' was included with a value of 1 for 'Goldrich', 0.5 value for 'Goldrich $x$ X' hybrids and null value for the other genotypes nonrelated to 'Goldrich'. Model parameters were estimated with a $95 \%$ confidence level $(P \leq 0.05)$.

\section{III.4. Results}

\section{III.4.1. Total polyphenols content}

The polyphenol content in plants varies depending on the part of the plant and the tissue. In the first year of the study, we analysed the polyphenol content on flesh and peel. Results showed the content in peel was about 8 to 10 fold than flesh (Figure 11). From the results obtained, in the next crop years the analysis was focused on peel, since there is the main contributor on polyphenols of the fruit. Taking into account that fresh and dried apricots are consumed with peel, this is the part of the fruit most important for assessing antioxidant capacity.

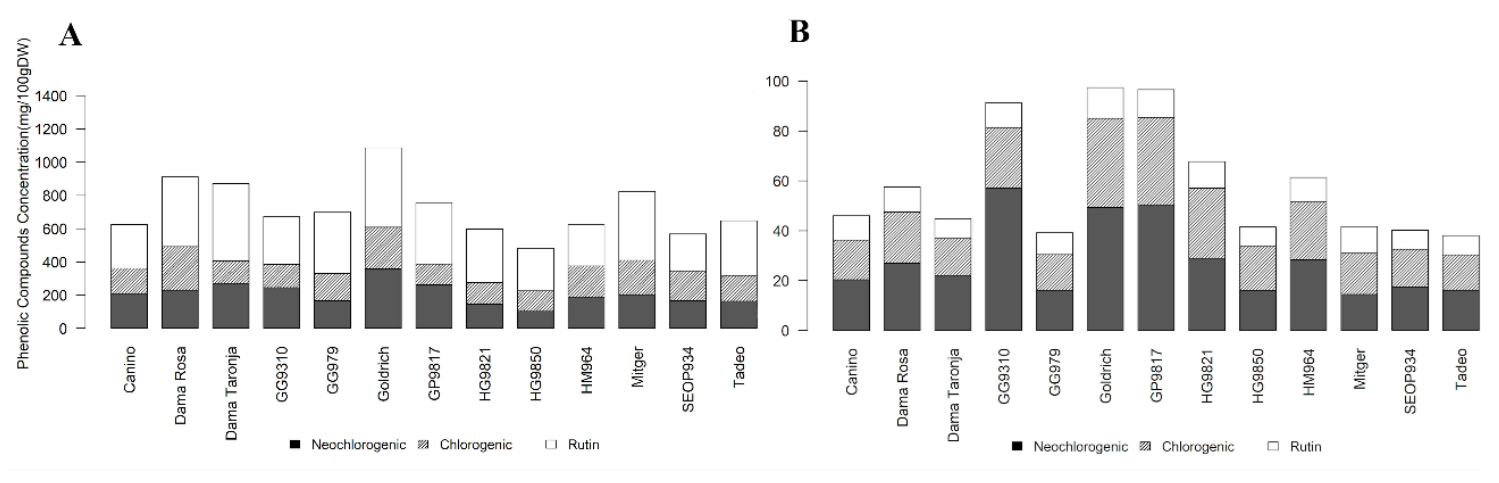

Figure 11. Polyphenol compounds concentration: Neochlorogenic acid, chlorogenic acid and Rutin. Data from 2016. A: Concentration in peel; B: Concentration in flesh.

The total polyphenol content of the varieties and selections studied varied among genotypes and years (Table 11 and Figure S1). Interestingly, the variety 'Goldrich', used in the breeding program as donor of resistance to PPV, has the highest content of total polyphenols, followed by 'Dama Rosa', a seedling from 'Goldrich' registered from the program and characterized by more than $80 \%$ of red blush peel. Both varieties showed an average of total polyphenols higher than $850 \mathrm{mg} / 100 \mathrm{~g}$ DW. A second group 
with more than $700 \mathrm{mg} / 100$ DW on average included the variety 'Canino' and the hybrids 'GG9310', 'GP9817', both seedlings from 'Goldrich' and the hybrids 'SEOP934' and HM966, this group resulted very rich in polyphenols. The year effect was relevant in the total content of polyphenols being the 3rd year the one in which the content was lower in $70 \%$ of the varieties studied (Table S7).

Table 11. Phenolic compounds: Neochlorogenic, chlorogenic, rutin and quercetin-3-glucuronide. 3years average \pm standard deviation. Different letter means significant differences among genotypes.

\begin{tabular}{|c|c|c|c|c|}
\hline Genotype & Neochlorogenic acid & Chlorogenic acid & Rutin & $\begin{array}{l}\text { Quercetin-3- } \\
\text { glucuronide }\end{array}$ \\
\hline 'Canino' & $174.43 \pm 53.13 a b c$ & $110.28 \pm 38.94 a$ & $420.16 \pm 238.55 a$ & $73.34 \pm 13.62 \mathrm{a}$ \\
\hline 'Dama Rosa' & $242.59 \pm 68.12 \mathrm{bcd}$ & $264.24 \pm 117.33 b$ & $316.02 \pm 134.03 a$ & $57.26 \pm 22.74 a$ \\
\hline 'Dama Taronja' & $216.28 \pm 77.50 \mathrm{abcd}$ & $166.22 \pm 96.88 a b$ & $257.27 \pm 141.18 \mathrm{a}$ & $75.06 \pm 41.35 a$ \\
\hline 'GG9310' & $278.97 \pm 44.54 \mathrm{~cd}$ & $131.47 \pm 15.22 \mathrm{a}$ & $324.27 \pm 140.95 a$ & $53.53 \pm 17.59 a$ \\
\hline 'GG979' & $160.31 \pm 19.75 a b$ & $165.08 \pm 31.40 a b$ & $241.45 \pm 134.84 a$ & $51.14 \pm 25.47 a$ \\
\hline 'Goldrich' & $297.43 \pm 111.09 \mathrm{~d}$ & $263.97 \pm 109.64 b$ & $388.92 \pm 85.30 \mathrm{a}$ & $79.11 \pm 26.37 a$ \\
\hline 'GP9817' & $236.79 \pm 73.99 \mathrm{bcd}$ & $175.80 \pm 84.08 a b$ & $293.97 \pm 67.30 \mathrm{a}$ & $48.33 \pm 16.59 a$ \\
\hline 'HG9821' & $162.66 \pm 16.52 a b$ & $126.27 \pm 31.78 a$ & $289.51 \pm 117.55 a$ & $53.17 \pm 25.79 a$ \\
\hline 'HG9850' & $110.92 \pm 8.69 a$ & $130.46 \pm 11.03 \mathrm{a}$ & $212.63 \pm 52.60 \mathrm{a}$ & $33.60 \pm 30.95 a$ \\
\hline 'HM964' & $237.58 \pm 109.86 \mathrm{bcd}$ & $203.72 \pm 92.04 a b$ & $243.43 \pm 46.39 a$ & $60.71 \pm 12.51 \mathrm{a}$ \\
\hline 'Mitger' & $164.16 \pm 38.00 a b$ & $134.59 \pm 61.62 \mathrm{a}$ & $268.43 \pm 130.97 a$ & $53.18 \pm 23.73 a$ \\
\hline 'SEOP934' & $207.65 \pm 88.31 \mathrm{abcd}$ & $224.15 \pm 107.90 a b$ & $255.74 \pm 71.70 \mathrm{a}$ & $78.35 \pm 58.47 a$ \\
\hline 'Tadeo' & $139.32 \pm 20.76 a b$ & $123.23 \pm 29.97 \mathrm{a}$ & $375.03 \pm 127.49 a$ & $71.13 \pm 12.35 \mathrm{a}$ \\
\hline
\end{tabular}

\section{III.4.2. Polyphenols compounds}

Fruits present complex mixtures of polyphenols. The phenolics substances in fruits are mainly phenolic acids and flavonoids. The most important compounds obtained in terms of quantity were: neochlorogenic acid, chlorogenic acid and flavonoids, as rutin and quecertin-3 glucuronide.

\section{III.4.2.1. Neochlorogenic acid}

Neochlorogenic acid concentration results revealed significant differences among accessions (Table 11). 'Goldrich' showed one of the highest concentrations on average and during the three years of sampling. The accessions with higher neochlorogenic acid content were the same that those with maximum polyphenol content. Neochlorogenic acid is one of the most relevant components of the 
total polyphenols according to quantity, being the most contributors to the polyphenol content in apricot. Neochlorogenic concentration within accessions was year dependent. A trend observed was a general lower concentration in all genotypes during the crop year 2018. Only 2 hybrids, 'HG9821' and 'HG9850' present the lowest content in 2016 year. Both hybrids are siblings from the same cross (Table S8).

\section{III.4.2.2. Chlorogenic acid}

Results of chlorogenic acid content average of the three crop years studied ranged between 110 to $277 \mathrm{mg} / 100 \mathrm{~g}$ DW (Table 11). The variety 'Goldrich' shows the maximum content. The variety 'Dama Rosa' and the hybrids 'GP9817', 'HM964' and 'SEOP934' showed content higher of $200 \mathrm{mg} / 100 \mathrm{~g}$ DW. Results into the different crop years showed differences among varieties and a similar trend than the observed in neochlorogenic acid (Table S9). The crop year 2018 resulted in the lower content of the 3 crop years studied in most of the varieties, except two hybrids 'HG9821' and 'HG9850', similarly to the results on neochlorogenic content.

\section{III.4.2.3. Rutin}

Results of rutin from the 3 crop years showed the variety "Canino' a traditional Mediterranean variety, with the highest content on average. The varieties in which the content was higher than $300 \mathrm{mg} / 100 \mathrm{~g}$ DW were 'Goldrich', "Dama Rosa' and "Tadeo'. Rutin concentration was no year-dependent (Table 11, Table S10). The trend detected of lower phenolic acids content in 2018 crop year was not observed in the content of rutin.

\section{III.4.2.4. Quercetin-3-glucuronide}

Results of quercetin-3-glucuronide average content in the three crop years analysed ranged between 33, 7 to 78,6 from the hybrid 'HG9850' and 'Goldrich' respectively (Table 11, Table S11). On the other hand, no significant differences were detected among years. The variety 'Goldrich' is one of the varieties with higher content among the set during the 3 crop years, which indicates it can be good parental for increasing the content of this compound in apricot by breeding.

\section{III.4.3. Principal components analysis}

Principal components analysis (PCA) was performed. (Table 12). Data revealed that $81.78 \%$ of variance was explained by the two first principal components. All the studied variables had positive scores for PC1. Distribution of varieties and hybrids studied plotted in the space of the first two PC is showed in Figure 12. 


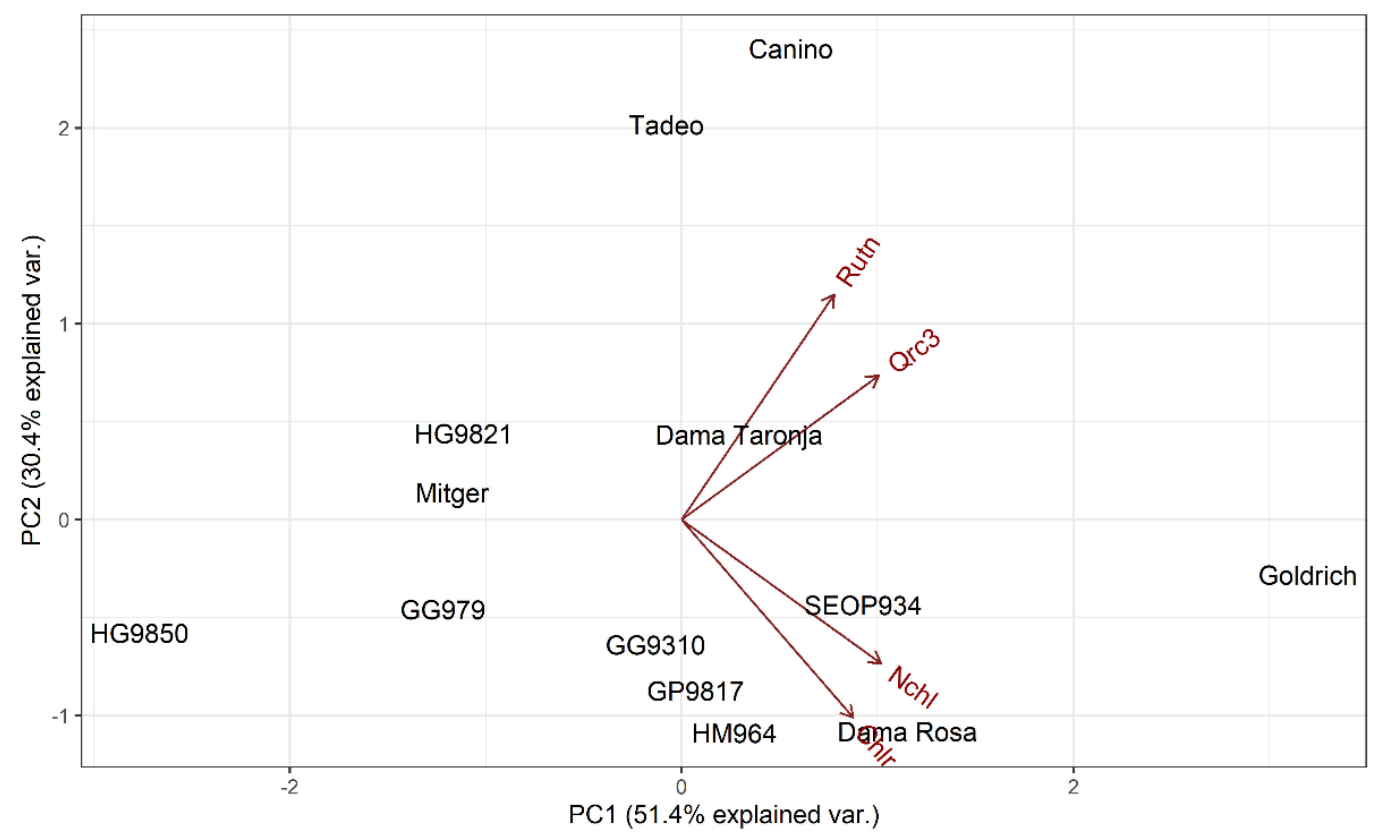

Figure 12. Plot of the variables studied and accessions in the space defined by the two first PC.

The accessions with higher polyphenol acid content are located in the positive scores of PC1 and negative of PC2. The variety with higher scores is 'Goldrich' which indicates that might be a good candidate for increasing the polyphenols acids in a breeding program. On the other hand, the content of polyphenols from the flavonoid group (rutin and quercetin-3-glucuronide) has positive values in PC1 and PC2. The varieties with higher PC2 scores are two traditional varieties well known "Canino' and 'Tadeo'.

Table 12. Variable contribution to Principal Components, eigenvalues, and cumulative variance in the PCA.

\begin{tabular}{lrrrr}
\hline \multirow{2}{*}{ Variable } & \multicolumn{4}{c}{ PCA } \\
\cline { 2 - 5 } & PC1 & PC2 & PC3 & PC4 \\
\hline Neochlorogenic acid & 0.55 & -0.40 & 0.35 & 0.64 \\
Chlorogenic acid & 0.47 & -0.55 & -0.52 & -0.46 \\
Rutin & 0.42 & 0.62 & -0.57 & 0.34 \\
Quercetin-3-glucuronide & 0.54 & 0.40 & 0.53 & -0.51 \\
\hline Eigenvalue & 2.06 & 1.22 & 0.40 & 0.32 \\
Variance (\%) & 51.38 & 30.40 & 10.10 & 8.12 \\
\hline Cumulative Variance (\%) & 51.38 & 81.78 & 91.88 & 100.00 \\
\hline
\end{tabular}


III.4.4. Contribution of the resistant cultivar 'Goldrich' to the quality traits studied.

In the frame of the breeding program all the genotypes studied were characterised according to the main pomological characteristics during the procedure of selection. Among the pomological traits we selected size and firmness of the fruit as traits that contribute to the quality. Table 13 indicates the obtained coefficients of the linear model related to the contribution of 'Goldrich' in the variables

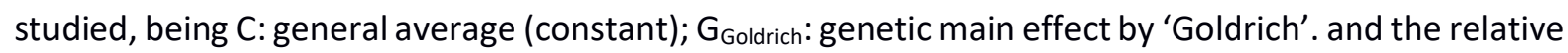
effect $\mathrm{G}_{\text {Goldrich }} / \mathrm{C}$.

Table 13. General Linear Model for phenolic compounds and pomological traits to test the 'Goldrich' effect and interaction. SSi: Sum of Squares; SS relative: SSi/SStotal; Year: environmental effect due to the year; G69: genetic main effect of 'Goldrich'; Year x G6969

\begin{tabular}{|c|c|c|c|c|c|c|c|c|c|c|}
\hline \multirow[b]{2}{*}{ Parameter } & \multicolumn{2}{|c|}{ Year } & \multicolumn{2}{|c|}{$\mathbf{G}_{\text {Goldrich }}$} & \multicolumn{2}{|c|}{ Year $\times \mathbf{G}_{\text {Goldrich }}$} & \multicolumn{2}{|c|}{ Residual } & \multirow[b]{2}{*}{$\mathrm{SS}_{\text {total }}$} & \multirow[b]{2}{*}{$\mathbf{R}^{2}$} \\
\hline & SSY $_{Y}$ & $\begin{array}{c}\text { SS } \\
\text { relative }\end{array}$ & $\mathbf{S S}_{\mathrm{G}}$ & $\begin{array}{c}\text { SS }_{\mathrm{G}} \\
\text { relative }\end{array}$ & $S S_{Y \times G}$ & $\begin{array}{c}S_{Y_{X G}} \\
\text { relative }\end{array}$ & $S_{S_{R}}$ & $\begin{array}{c}S_{R} \\
\text { relative }\end{array}$ & & \\
\hline Neochlorogenic & $50558 * *$ & 0.073 & $174553^{* *}$ & 0.250 & $32397.6 * *$ & 0.046 & 316370 & 0.454 & 696869 & 0.546 \\
\hline Chlorogenic & $30924.2^{*}$ & 0.037 & $101004^{* *}$ & 0.122 & $71525.1^{* *}$ & 0.086 & 473383 & 0.571 & 828517 & 0.428 \\
\hline Rutin & 4083.31 & 0.002 & 61965.9 NS & 0.028 & 68295.8 NS & 0.031 & $1.98 \cdot 10^{6}$ & 0.900 & $2.20 \cdot 10^{6}$ & 0.100 \\
\hline $\begin{array}{l}\text { Quercetin-3- } \\
\text { glucurunide }\end{array}$ & $14236.6 * *$ & 0.164 & $594.246 \mathrm{NS}$ & 0.007 & $1772.51 \mathrm{NS}$ & 0.020 & 54330.4 & 0.627 & 86715.6 & 0.373 \\
\hline Height (mm) & $472.148 * *$ & 0.085 & $628.233^{* *}$ & 0.113 & 11.936 NS & 0.00214 & 3951.410 & 0.709 & 5572.190 & 0.291 \\
\hline Diameter(mm) & $667.365^{* *}$ & 0.133 & 31.518 NS & 0.006 & $67.731 \mathrm{NS}$ & 0.01346 & 3578.150 & 0.711 & 5032.970 & 0.289 \\
\hline \multicolumn{11}{|l|}{ Ratio } \\
\hline$\frac{\text { Height }}{\text { Diameter }}$ & $1,341 * *$ & 0,104 & $0,338^{*}$ & 0,026 & $0,055 \mathrm{NS}$ & 0,004 & 9,909 & 0,770 & 12,870 & 0,230 \\
\hline Weight (fruit) & $6867.690 *$ & 0.113 & 1044.740 & 0.017 & 557.715 NS & 0.00918 & 44907.600 & 0.739 & 60771.60 & 0.261 \\
\hline Weight(stone) & 3.094 NS & 0.018 & $26.946 * *$ & 0.157 & $1.733 \mathrm{NS}$ & 0.01009 & 132.377 & 0.771 & 171.7200 & 0.229 \\
\hline \multicolumn{11}{|l|}{ Ratio } \\
\hline \multicolumn{11}{|l|}{$\overline{\text { weight(stone) }}$} \\
\hline $\begin{array}{l}\text { Firmness } \\
\left(\mathrm{kgf} / \mathrm{cm}^{2}\right)\end{array}$ & $85.560 * *$ & 0.173 & $29.321^{* *}$ & 0.059 & 13.592 NS & 0.02752 & 359.382 & 0.728 & 493.834 & 0.272 \\
\hline
\end{tabular}

${ }^{*}$ Significant differences $(\mathrm{P} \leq 0.05)$; **Significant differences $(\mathrm{P} \leq 0.01)$; NS: non-significant.

Among the phenolic compounds, neochlorogenic and chlorogenic acids showed a significant genetic effect of 'Goldrich' (contribution of 25 and $12.2 \%$ of total sum of squares, respectively). However, nonsignificative contribution was observed in rutin and quercetin-3-glucuronide. The linear model coefficients were calculated for 'Goldrich' genetic effect in the accumulation of the studied phenolic 
compounds (Table 14). The value for neochlorogenic was $121.8 \mathrm{mg} / 100$ ( $71 \%$ of general average) and for chlorogenic acid $92.6 \mathrm{mg} / 100 \mathrm{gDW}$ (63.5\% of general average) These results indicate an important contribution of this variety to these polyphenol acids.

Table 14. Variables studied and 'Goldrich' contribution. C: General average value of the population studied. $G_{\text {Goldrich: }}$ 'Goldrich' contribution. $G_{\text {Goldrich }}$ relative: Relative contribution of 'Goldrich' to the general average. Confidence intervals at $95 \%$.

\begin{tabular}{l|c|c|c}
\hline \multicolumn{1}{c}{ Parameter } & C & GGoldrich & $\begin{array}{c}\text { GGoldrich } \\
\text { relative }\end{array}$ \\
\hline Neochlogenic & $170.2 \pm 12.8$ & $121.8 \pm 30.8^{* *}$ & 0.72 \\
Chlorogenic & $145.8 \pm 15.7$ & $92.6 \pm 37.7^{* *}$ & 0.64 \\
Rutin & $284.6 \pm 32.1$ & $72.6 \pm 77.1$ & 0.25 \\
Quercetin-3-glucurunide & $58.7 \pm 5.3$ & $7.1 \pm 12.8$ & 0.12 \\
Height (mm) & $41.5 \pm 1.0$ & $6.3 \pm 2.5^{* *}$ & 0.15 \\
Diameter(mm) & $47.0 \pm 1.0$ & $1.4 \pm 2.4 \mathrm{NS}$ & 0.03 \\
Ratio $\frac{\text { Height }}{\text { Diameter }}$ & $1.2 \pm 0.1$ & $0.1 \pm 0.1^{*}$ & 0.13 \\
Weight (fruit) (g) & $55.6 \pm 3.4$ & $8.2 \pm 8.4 \mathrm{NS}$ & 0.15 \\
Weight(stone) (g) & $2.8 \pm 0.2$ & $1.3 \pm 0.5^{* *}$ & 0.48 \\
Ratio $\frac{\text { weight(fruit) }}{\text { weight(stone) }}$ & $20.4 \pm 0.9$ & $-5.1 \pm 2.2^{* *}$ & -0.25 \\
& & & -0.57 \\
\hline Firmness (kgf/cm $\left.{ }^{2}\right)$ & $2.5 \pm 0.3$ & $-1.4 \pm 0.8^{* *}$ & \\
\hline
\end{tabular}

*Significant differences $(\mathrm{P} \leq 0.05)$; **Significant differences( $\mathrm{P} \leq 0.01)$; NS: non-significant parameter.

In pomological traits related to size and weight of the fruit, the genetic contribution of 'Goldrich' was significant as well (Tables 13 and 14). However, the contribution in firmness is negative, being $1.4 \mathrm{kgf} / \mathrm{cm} 2$ (57\% less of general average). This result indicates that 'Goldrich' might decrease the firmness of the fruits in the progenies.

\section{III.5. Discussion}

\section{III.5.1. Total polyphenol content}

Recent studies pointed out the antioxidant content of fruits as one of the main attributes to promote fruit consumption. Breeding for fruit quality should take into account the increase of those compounds with antioxidant activity. 
Several studies shown phenolic compounds distribution depends on tissues, being higher in peel than in pulp (Campbell and Padilla-Zakour, 2013). In fruits polyphenols have been located in flesh and peel. In many fruits analysed the content in peel is higher than in flesh. In the present study the content of all compounds analysed was more than 10 fold in peel than in flesh, in agreement with results in other studies focused on plum, peach and apricot (Veberic and Stampar, 2005). This fact has been explained because of their role in defence against ultraviolet radiation, protection in front of pathogens and environmental stress (Manach et al., 2004). Since apricot is consumed with peel in all ways of consumption, fresh, dried, and canning, the content of polyphenols of apricot becomes one of the most important attributes of fruit quality. The fruit consumption is decreasing in the EU 28 , hence the apricots fruits as a source of antioxidants, could be used for encouraging their consumption.

The phenolic acids studied as well the flavonoids derivates are secondary metabolites related to different functions including pigments and antioxidant activity. Polyphenol genetic control have been studied in model plants and some relevant genes have been identified. In Arabidopsis, a phenylalanine ammonia-lyase (PAL) has been identified as involved in the first step of the phenylpropanoid pathway (Fraser and Chapple, 2011). Other genes associated to anthocyanin accumulation were dihydroflavonol 4-reductase (DFR) and flavonol synthase (FLS) (Jin et al., 2016; Luo et al., 2016). In apricot by means of a transcriptomic approach MYB10 gene was proposed as the best candidate for skin colour (García-Gómez et al., 2019), however, there is still a lack of information of the genes and mechanisms involved in the anthocyanin pathway for using them in molecular breeding.

Their concentrations in foods vary according to numerous genetic and environmental factors (Manach et al., 2004; Mole et al., 1988). In this study, the genetic effect was indicated by the differences among genotypes and the environment effect was analysed by means of sampling in 3 crop years. An important effect of lower general content of polyphenols during crop year 2018 was observed. Since the polyphenols synthesis and accumulations occurs during maturity of the fruit, the ripening process is being close related to polyphenol accumulation (Kennedy et al., 2000). In our study since the varieties share the same location, crop management and laboratory conditions the differences observed between years might be due to differences in climatic conditions among years.

Several studies have shown that chlorogenic and neo-chlorogenic acids are related to some biological activities in which the antioxidant and antimicrobial properties are very relevant (Dillard and German, 2000; Jin et al., 2005; Sabu and Kuttan, 2002). The range of values obtained in apricot for both compounds was similar to those described in read plum skin (Stacewicz-Sapuntzakis et al., 2001), which indicates that apricot species is a good source of polyphenols acids. In apricot, a similar to plum range of concentrations of chlorogenic acid was found (Gündoğdu et al., 2013; Ruiz et al., 2005) in agreement with our results. 
Concerning to the amount of rutin content in apricot, similar results were obtained by Fan et al. (2018) and Gündoğdu et al. (2013). Rutin is the glycoside form of quercetin and it has been related as well with antioxidant and antimicrobial properties and due to its chemical structures are related with others beneficial health processes. Due to the high content of these compounds in apricot, some studies suggested that apricot is a good source of phytochemicals with antioxidant potential (Fan et al., 2018). Concerning to quercetin-3-glucuronide, the range of content obtained was similar as described in other species (Nicolle et al., 2004). Additionally, this compound had the higher contribution to antioxidant activity in apricots (Fan et al., 2018).

\section{III.5.2. Contribution of the PPV-resistant 'Goldrich' variety to fruit quality}

Since the spread of sharka diseases, the production of apricot in the main producing areas of Europe and the Mediterranean Basin are based on varieties obtained by breeding (Bassi et al, 2010; Egea et al, 2010; Karayiannis, 2006a and 2006b; Martinez-Calvo et al, 2009; Pennone et al, 2010). In Central Europe the resistant varieties from Ontario, such as 'Henderson' and 'Harlayne' were well adapted (Polak et al., 2008) but it was not the case in the European Southern regions as Spain and Italy in which the crop needs medium chilling varieties. Among the different resistant cultivars 'Goldrich' was the less affected for the lack of chilling.

Results from this study showed that 'Goldrich' is a good contributor for increasing antioxidant content, its genetic effect represented up to 65 to $70 \%$ of the total average, which indicated a relevant role in increasing polyphenolic compounds compared to the other cultivars studied. This fact pointed out that crosses involving this variety are even more relevant for increasing the polyphenol content of the seedlings than the other genotypes studied.

\section{III.6. Conclusions}

The set of apricot accessions analysed showed different contain in the polyphenols compounds. The content was genetic and environment dependent. Concentration of polyphenols in apricot peel is 10 fold higher than flesh, since this fruit is consumed with peel in the different ways, fresh and dried, this trait is relevant for increasing the apricot consumption. The cultivar 'Goldrich' used as a donor of resistance to sharka diseases at different breeding programs, including the IVIA's program, resulted the variety with highest contribution to the polyphenol content among the accessions studied. The genetic effect of 'Goldrich' in this trait indicated it was a good candidate for increasing both neochlorogenic and chlorogenic acid content of fruits in the breeding program. The comparison of the first generation of 'Goldrich' hybrids with other genotypes shows that 'Goldrich' remains as a good parental for increasing the antioxidant content of apricot by breeding, which would increase as well the fruit quality. 


\section{CHAPTER IV: Insights of phenolic pathway in fruits: transcriptional and metabolic profiling in apricot (Prunus armeniaca)}

This section is based on an article published in International Journal of Molecular Sciences (2021, 22(7), 3411). The final authenticated version is available online at:

https://doi.org/10.3390/ijms22073411

Author contribution:

- Helena Gómez-Martínez: exprerimental procedures, statistical analysis, data curation, writing original draft, review and editing.

- F. Gil-Muñoz: experimental procedures and statistical analysis, writing original draft, review and editing.

- Bermejo: Analysis of polyphenols.

- $\quad$ E. Zuriaga: Conceptualization, writing original draft, review, and editing

- M.L. Badenes: Conceptualization, funding acquisition, writing original draft, review, and editing. 


\section{IV.1. Abstract}

There is an increasing interest in polyphenols, plant secondary metabolites, in terms of fruit quality and diet, mainly due to its antioxidant effect. However, the identification of key gene enzymes and their roles in the phenylpropanoid pathway in temperate fruits species remains uncertain. Apricot (Prunus armeniaca) is a Mediterranean fruit with high diversity and fruit quality properties, being an excellent source of polyphenol compounds. For a better understanding of the phenolic pathway in those fruits, we selected a set of accessions with genetic-based differences in phenolic compounds accumulation. HPLC analysis of the main phenolic compounds and transcriptomic analysis of the genes involved in key steps of the polyphenol network were carried out. Phenylalanine ammonia-lyase (PAL), dihydroflavonol-4-reductase (DFR) and flavonol synthase (FLS) were the key enzymes selected. Orthologous of the genes involved in transcription of these enzymes were identified in apricot: ParPAL1, ParPAL2, ParDFR, ParFLS1 and ParFLS2. Transcriptomic data of the genes involved in those critical points and its relationships with the polyphenol compounds were analyzed. Higher expression of ParDFR and ParPAL2 has been associated to red-blushed accessions. Differences in expression between paralogous were linked to the presence of a BOXCOREDCPAL cis-acting element related to the genes involved in anthocyanin synthesis ParFLS2, ParDFR and ParPAL2.

\section{Keywords:}

Phenolic pathway; FLS; DFR; PAL; fruits 


\section{IV.2. Introduction}

Apricot (Prunus armeniaca) is an important fruit crop in Mediterranean basin countries and Asia, with a wide diversity in pomological characteristics and fruit quality properties due to its different diversification centres (Bailey and Hough, 1975). Apricots are a good source of vitamins, carotenoids, and polyphenols (Roussos et al., 2011); which makes this species a good choice from a nutraceutical point of view (Ruiz and Egea, 2008).

Higher plants have several defence mechanisms against biotic and abiotic stresses. Some of these mechanisms result in the synthesis of a large number of secondary metabolites. Flavonoids are one of these defence-related secondary metabolites, being a family of polyphenols synthesized by phenylpropanoid biosynthetic pathway (Ferrer et al., 2008). These secondary metabolites remain in different plant organs and accumulate on the plant surface (Harborne, 2009). In the case of flavonoid compounds, its accumulation is unequally distributed within tissues, being its concentration higher in the peel of several fruits such as apple (Bizjak et al., 2013), peach (Campbell and Padilla-Zakour, 2013) or apricot (Gómez-Martínez et al., 2021).

Polyphenols have been identified as secondary metabolites with great antioxidant activity (Fu et al., 2010; Gan et al., 2016; Mokrani et al., 2016). In recent years, there is an increasing interest in them as contributors to the fruit quality and dietary properties. In the case of apricot, the fruit peel is an excellent source of phenolic compounds. Main phenylpropanoid-derivate secondary metabolites in apricot are chlorogenic and neochlorogenic acids, two caffeate derivates monolignols, while main flavonols are rutin and quercetin-3-glucuronide (Erdogan-Orhan and Kartal, 2011).

Phenylpropanoid biosynthesis starts from the conversion of L-phenylalanine into cinnamic acid due to the action of phenylalanine ammonia-lyase (PAL) (Figure 13). Phenylalanine ammonia-lyase (PAL) has been described as the first enzyme in the phenylpropanoid pathway, being considered as a key regulatory point between primary and secondary metabolism through conversion of L-phenylalanine into cinnamic acid (Fraser and Chapple, 2011). PAL is encoded by a multi-gene family, in which the number of genes involved depends on the species. In Arabidopsis and Nicotiana four PAL-encoding genes have been described (Fukasawa-Akada et al., 1996; Raes et al., 2003; Reichert et al., 2009), five in poplar (Hamberger et al., 2007), and two in different Prunus species (Irisarri et al., 2016). In the following step, cinnamic acid 4-hydroxylase converts cinnamic acid into p-coumaric acid, to which a coenzyme-A is added due to the action of 4-coumarate-CoA ligase, giving a $\rho$-coumaroyl-CoA as a result. At this point, the pathway can branch off to the caffeate derivates biosynthesis, producing chlorogenic and neochlorogenic acids. Alternatively, $\rho$-coumaroyl-CoA is also used by chalcone synthase to catalyse the synthesis of chalcone, which is isomerized to colourless flavanones. These compounds can be hydroxylated at three different positions, by three different flavonoid hydroxylases, 
producing a group of dihydroflavonols. Then, the phenolic pathway can branch off to the flavonols biosynthesis due to the action of flavonol synthase (FLS). This enzyme uses dihydroflavonols (dihydroquercetin, dihydrokaempferol or dihydromyricetin) as a substrate to produce kaempferol, quercetin, or myricetin the main precursors of some flavonols such as rutin or quercetin-3-glucuronide. Previous works have identified FLS-encoding genes in Arabidopsis (Owens et al., 2008; Pelletier et al., 1999). In addition, FLS has been related with dihydroflavonols catalysis to flavonol but also it has been related with anthocyanin accumulation (Kuhn et al., 2011; Owens et al., 2008). On the other hand, dihydroflavonol-4-reductase (DFR) enzyme controls one of the limiting steps of the anthocyanin pathway reducing dihydroflavonols to leucoanthocyanidins (Lo Piero et al., 2006; Martens et al., 2003; Shimada et al., 2005), therefore using the same substrate as FLS. Several DFR-encoding genes have been identified in different species (Huang et al., 2012; Shimada et al., 2005; Singh et al., 2009; Xie et al., 2004). Although phenolic metabolism regulation remains ambiguous in some points, various studies have identified the role of MYB transcription factors in phenolic synthesis regulation (Hartmann et al., 2005; Jin et al., 2016; Luo et al., 2016).

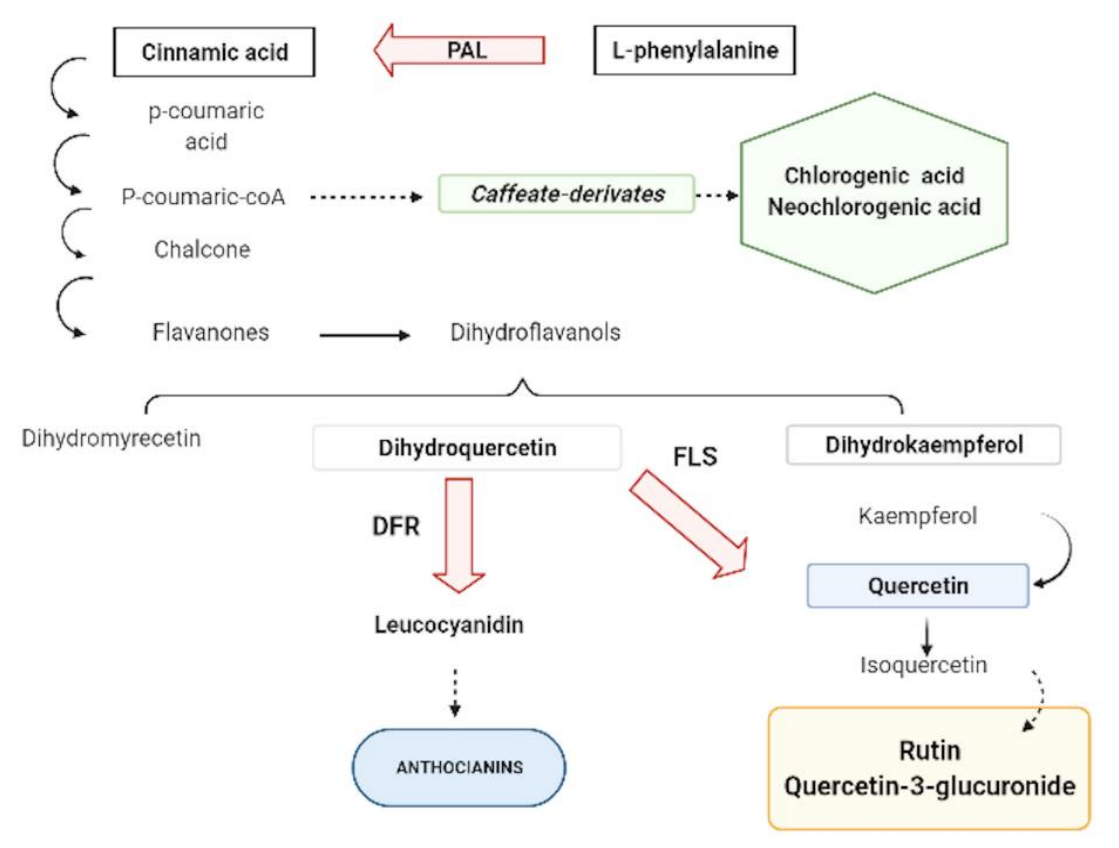

Figure 13. Phenolic biosynthesis pathway.

Although the main steps of the metabolic pathway are described, the identification of key gene enzymes and their roles in the phenylpropanoid pathway of some fruit crops, remains uncertain. As the first step for a better understanding of the phenolic pathway in fruits, we selected a set of apricot accessions from the IVIA's apricot breeding program with genetic-based differences in phenolic 
compound accumulation (Gómez-Martínez et al., 2021). Fruit phenolic content of the genotypes selected was evaluated and compared with the genetic expression of genes encoding key enzymes of phenolic biosynthesis pathway related to primary phenolic compounds (PAL), anthocyanin biosynthesis (DFR) and secondary phenolic metabolites (FLS). Since FLS and DFR use the same substrate for producing either flavonols or anthocyanins, respectively, their possible role in flavonol accumulation in apricot should be studied. Characterization of the expression of main genes acting in the phenolic pathway and its relationship with fruit polyphenol content will provide tools for unravel the phenolic pathway of fruit species aimed at further breeding on fruit quality and promotion of fruit consumption.

\section{IV.3. Materials and Methods}

\section{IV.3.1. Plant material}

A set of 2 Mediterranean cultivars ("Canino' and 'Mitger') a North American variety ('Goldrich') and 9 hybrids from the IVIA's apricot breeding program were analysed (Table 15). 'Goldrich' used as the main donor of resistance to PPV at the breeding program is one of the parents in most of the resistant hybrids obtained. 'Canino' and 'Mitger' are two autochthonous varieties used for introgression of adaptability to the Mediterranean conditions. The trees are maintained at the IVIA's apricot collection

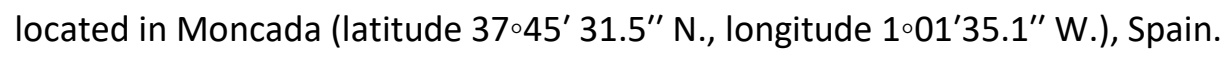

Table 15. Plant material used in the study, pedigree, and origin.

\begin{tabular}{lll}
\hline Genotype & Pedigree & Origin \\
\hline 'Canino' & Unknown & \\
'Dama Rosa' & Goldrich x Ginesta & Spain \\
'Dama Taronja' & IVIA \\
'GG9310' & Goldrich x Katy & IVIA \\
'GG979' & Goldrich x Ginesta & IVIA \\
Goldrich & Sunglo x Perfection & USA \\
'GP9817' & Goldrich x Palau & IVIA \\
'HG9821' & Harcot x Ginesta & IVIA \\
'HG9850' & Harcot x Ginesta & IVIA \\
'HM964' & Harcot x 'Mitger' & IVIA \\
'Mitger' & Unknown & Spain \\
'SEOP934' & SEO x Palau & IVIA \\
\hline
\end{tabular}


Five fruits per tree were harvested at the ripening stage during two growing seasons (2019 and 2020). For each fruit, the peel was separated from the flesh with a peeler. The samples consisted of a mix of the peel from 5 fruits per genotype and year. Samples were frozen with liquid nitrogen and kept at $80^{\circ} \mathrm{C}$ until processing.

\section{IV.3.2. HPLC analysis}

For HPLC analysis, the tissue was processed to lyophilized powder. Tissue homogenization was carried out using a vortex. Phenolic compounds were extracted and determined according to the procedure described by Cano et al., (2008) and Cano and Bermejo (2011). Briefly, $10 \mathrm{mg}$ of freeze-dried peel were mixed with $1 \mathrm{~mL}$ of DMSO/MeOH (1:1, v/v). Then the sample was centrifuged (Eppendorf 5810R centrifuge; Eppendorf Iberica, Madrid, Spain) at $4 \stackrel{\circ}{ } \mathrm{C}$ for $20 \mathrm{~min}$ at $10000 \mathrm{rpm}$. The supernatant was filtered through a $0.45 \mu \mathrm{m}$ nylon filter and analysed by HPLC-DAD and HPLC-MS in a reverse-phase column C18 Tracer Excel 5 rm 120 OSDB (250 mm x 4.6 mm) (Teknokroma, Barcelona, Spain). An Alliance liquid chromatographic system (Waters, Barcelona, Spain) equipped with a 2695 separation module, coupled to a 2996 photodiode array detector and a ZQ2000 mass detector was used. A gradient mobile phase consisting of acetonitrile (solvent A) and $0.6 \%$ acetic acid (solvent B) was used at a flow rate of $1 \mathrm{~mL} / \mathrm{min}$, with an injection volume of $10 \mu \mathrm{L}$. The gradient change was as follows: 10 $\% 2 \mathrm{~min}, 10-75 \% 28 \mathrm{~min}, 75-10 \% 1 \mathrm{~min}$, and hold at $10 \% 5 \mathrm{~min}$. An HPLC-MS analysis was performed and worked under electrospray ion positive (flavonoids) and negative (phenolic acids) conditions. Capillary voltage was $3.50 \mathrm{kV}$, cone voltage was $20 \mathrm{~V}$, source temperature was $100 \stackrel{\circ}{ }{ }^{\circ}$, desolvation temperature was $225 \stackrel{\circ}{\circ}$, cone gas flow was $70 \mathrm{~L} / \mathrm{h}$.

\section{IV.3.3. Obtention of gene sequences and cis-acting elements motif identification}

To identify the genetic regulation in the phenolics biosynthesis pathway, a set of genes encoding for dihydroflavonol-4-reductase (DFR), flavonol synthase $(F L S)$ and phenylalanine ammonia-lyase (PAL) were selected. To obtain putative orthologs of apricot species, a BLAST search was performed using $A$. thaliana and $P$. persica described genes in GDR (Genome Rosaceae Database, https://www.rosaceae.org/ / on Prunus armeniaca genome.

Identification of cis-acting elements was made from a total sequence of $1500 \mathrm{bp}$ upstream of the start codons from the Prunus armeniaca genome published at Genomic Database of Rosaceae (GDR). Analysis of cis-acting elements was made using PLACE (Plant cis-acting Elements) database (Higo et al., 1999) and searching for described motifs related to phenolic pathway.

In addition, to check the sequence conservation among species, a phylogenetic analysis was made with the obtained Prunus armeniaca genes predicted proteins and Prunus persica (PpeDFR (Prupe.1G376400.1), PpeFLS1 (Prupe.1G502700.1), PpeFLS2 (Prupe.1G502800.1), PpePAL1 
(ppa002328m), PpePAL2 (ppa002099m)), Fragaria vesca (FvDFR (mrna15174.1-v1.0-hybrid), FvFLS1 (mrna11126.1-v1.0-hybrid), FvPAL1 (mrna23261.1-v1.0-hybrid), FvPAL2 (mrna09753.1-v1.0-hybrid)), Vitis vinifera (VvDFR (GSVIVT01009742001), VvFLS1(GSVIVT01008913001), VvPAL1 (GSVIVT01016257001)), Malus domestica (MdDFR (MDP0000734274), MdFLS1 (MDP0000311541), MdFLS2 (MDP0000294667), MdPAL1 (MDP0000668828), MdPAL2 (MDP0000261492)) and Arabidopsis thaliana (AtDFR (NM_123645.4), AtFLS1 (U84259.1), AtFLS2 (BT003134.1), AtFLS3 (NM_125754.3), AtPAL1 (AY303128.1), AtPAL2 (AY303129.1), AtPAL3 (NM_001203294.1), AtPAL4 (AY303130.1)) predicted proteins. For apricot, coding sequences (ParDFR (PARG07267m); ParPAL1 (PARG18722m), ParPAL2 (PARG02214m), ParFLS1 (PARG08425m), ParFLS2 (PARG08426m), were translated into proteins with a DNA translate tool from Expasy (https://web.expasy.org/translate/). Multiple protein sequence alignment was performed with the ClustaIW program with MEGA X v.10.1.8 software, and a phylogenetic tree was built with the Neighbour-Joining method using MEGA X v.10.1.8 software with a bootstrap value of 1000 replicates.

The number of amino acid differences per site from between sequences ( $p$-distance) was calculated with MEGA X Software with bootstrap method with 1000 replications. 1- $p$-distance was calculated to similarity estimation among proteins. In addition, a BLAST and a synteny of Prunus persica against and Prunus armeniaca reference genome was performed in GDR database. Moreover, a BLAST of Arabidopsis thaliana against Prunus armeniaca genome was also performed in GDR database (Genome Rosaceae Database, https://www.rosaceae.org/).

\section{IV.3.4. Gene expression}

Samples consisted of $80 \mathrm{mg}$ of powered tissue. RNA isolation was made using Plant/Fungi Total RNA Purification Kit (NORGEN, Thorold, ON, Canada) with some modifications. Frozen power tissue was diluted in $600 \mathrm{~mL}$ of lysis buffer C, a $2 \%$ PVP-40 and $2 \% \beta$-mercaptoethanol was added. Purified RNA quality and integrity were checked by agarose gel electrophoresis, RNA was quantified by Qubit (Invitrogen, Carlsbad, CA, USA).

cDNA synthesis was obtained from 500ng of RNA diluted in $10 \mu \mathrm{L}$ reaction using the Primescript RT Reagent kit ('Perfect Real Time') (Takara Bio, Otsu, Japan).

Amplification was carried out with StepOnePLus Real-Time PCR System (Life Technologies, Carlsbad, CA, USA) software and TB Green Premix Ex Taq (Tli RNaseH Plus) (Takara Bio, Otsu, Japan) kit was used. Mix reaction contained $7.5 \mu \mathrm{L}$ enzyme, $0.09 \mu \mathrm{L}$ of primers [100 $\mu \mathrm{M}$ ], $0.3 \mathrm{~mL}$ ROX, $5.02 \mu \mathrm{L} \mathrm{H} 20$, and $1 \mu \mathrm{L}$ of cDNA. Mix was incubated at 95ㄷ for 30 seconds, followed by 40 cycles of 5 seconds at $95 \circ \mathrm{C}$ and 30 seconds at $60^{\circ} \mathrm{C}$. Finally, the mix was incubated for 15 seconds at $95^{\circ} \mathrm{C}$, followed by a minute at $60^{\circ} \mathrm{C}$ and 15 seconds at 95ㄷ. Apricot ACTIN and SAND as housekeeping genes. Primers used are indicated in Table 16. 
Table 16. Used primers in the studied and reference genes amplification.

\begin{tabular}{lcc}
\hline \multicolumn{1}{c}{ Gen } & Forward & Reverse \\
\hline ParPAL1 & CGACTGGGTTATGGATAGCATGA & CAATGTGTGGGTAGATTCTGTGC \\
ParPAL2 & TAAAGAGGTGGATAGTGCAAGGG & GAGAACACCTTGTCGCATTCTTC \\
ParfLS1F & TGGAGGGGATGACATGGTTTATC & CCGTTGCTCATAATCTCCATCTG \\
ParfLS2F & ACAGGAGGAAAAGGAGGCTTATG & GGCCAGAACCGGTAATTAATGAC \\
ParDFR & GTTCGAAGGCTGGTGTTTACATC & GAGAAATGGGCCAATCACAAGAG \\
ACTIN & CTTCTTACTGAGGCACCCCTGAAT & AGCATAGAGGGAGAGAACTGCTTG \\
SAND & TCGTGGGTACCAGGAAAACGACAT & CCTGCTAGCTTGTGTTCATCTCCA \\
\hline
\end{tabular}

\section{IV.3.5. Data analysis}

Data were statistically analysed by Statgraphics Centurion VII version 17.2.00 software (Statpoint Technologies Inc., Warrenton, VA, USA). Differences among samples and years were analysed with Kruskal-Wallis test $(P \leq 0.05)$ and averages were compared using the Multiple Range Test with Bonferroni method.

For testing the contribution of 'Goldrich' to the phenolic content and genetic expression in the set of accessions, we performed a regression of the data to a general linear model (Gómez-Martínez et al., 2021). In the model, the phenotype is linearly explained as follows:

$$
\text { Phenotype }=\mathrm{C}+\mathrm{G}_{\text {Goldrich }}+\text { Year }+\mathrm{G}_{\text {Goldrich }}{ }^{*} \text { Year }+ \text { Residual }
$$

Where $C$ is the general average of the population (constant), $G_{\text {Goldrich }}$ is the genetic effect of 'Goldrich', Year is the environmental effect due to the year and Residual is the residual effect. The model was calculated using the Statgraphics Centurion VII version 17.2.00 software (Statpoint Technologies, Warrenton, VA, USA). A quantitative variable for evaluating the genetic effect of 'Goldrich' was included with a value of 1 for 'Goldrich', 0.5 value for 'Goldrich $\times X^{\prime}$ ' hybrids, and a null value for the other genotypes non-related to 'Goldrich'. Model parameters were estimated with a $95 \%$ confidence level $(P \leq 0.05)$.

Elucidation of parameters significantly influent in phenolic content was made by a linear regression model with Statgraphics Centurion VII version 17.2.00 software (Statpoint Technologies, Warrenton, VA, USA). Parameters included in the linear regression were genetic expression in apricot of DFR, FLS1, FLS2, PAL1, and PAL2, and the following genetic expression ratios: PAL1/PAL2, PAL1/FLS1, PAL1/FLS2, PAL2/FLS1, PAL2/FLS2, and FLS1/FLS2. However, parameters with a $p$-value $>0.05$, were excluded from each model and only those significant were maintained.

In addition, a multivariate analysis was performed with Statgraphics XVII software (Statpoint 
Technologies, Warrenton, VA, USA) to study Pearson correlation among gene expression, phenolic contents, and relationships among all of them. Correlation with a $\mathrm{P}<0.05$ was considered significant. Graphics were made using R-studio software (Version1.1.463, 2009-2018, Rstudio, Inc.) with 'stats', grDevices', and 'graphics' (R Core Team), 'dplyr' (Wickham, 2021), 'readxl' (Wickham, 2016a), 'plyr' (Wickham, 2020), 'scales' (Wickham and Seidel, 2020) and 'ggplot2' (Wickham, 2016b) packages.

\section{IV.4. Results}

\section{IV.4.1. Apricot polyphenol content}

Total polyphenol content is indicated in Table 17. Significant differences were found among all genotypes studied. The higher values were obtained in genotypes with an important redblush colour on the skin.

Table 17. Polyphenol total content $(\mathrm{mg} / 100 \mathrm{gDW})$. Average \pm standard deviation. Different letter means significant differences among genotypes. Varieties with * produced fruits with a redblush of skin $>50 \%$.

\begin{tabular}{llll}
\hline Genotype & \multicolumn{1}{c}{$\mathbf{2 0 1 9}$} & \multicolumn{1}{c}{$\mathbf{2 0 2 0}$} & \multicolumn{1}{c}{ Two-years average } \\
\hline 'Canino' & $539,63 \pm 12,81 \mathrm{ab}$ & $669,52 \pm 30,03 \mathrm{a}$ & $604,58 \pm 74,08 \mathrm{a}$ \\
'Dama Rosa'* & $1725,21 \pm 222,12 \mathrm{~g}$ & $1565,50 \pm 64,26 \mathrm{e}$ & $1645,36 \pm 170,41 \mathrm{~d}$ \\
'Dama Taronja' * & $699,71 \pm 27,00 \mathrm{bcd}$ & $1171,19 \pm 286,97 \mathrm{~cd}$ & $935,45 \pm 316,10 \mathrm{abc}$ \\
'GG9310'* & $1024,98 \pm 9,58 \mathrm{ef}$ & $1008,08 \pm 80,72 \mathrm{abc}$ & $1016,53 \pm 52,23 \mathrm{bc}$ \\
'GG979'* & $630,10 \pm 11,57 \mathrm{abc}$ & $1018,73 \pm 159,22 \mathrm{bc}$ & $824,41 \pm 235,59 \mathrm{abc}$ \\
Goldrich & $894,02 \pm 25,15 \mathrm{de}$ & $876,18 \pm 22,06 \mathrm{abc}$ & $885,10 \pm 23,31 \mathrm{abc}$ \\
'GP9817'* & $1167,16 \pm 17,91 \mathrm{f}$ & $916,08 \pm 67,15 \mathrm{abc}$ & $1041,62 \pm 144,38 \mathrm{bc}$ \\
'HG9821' & $514,38 \pm 3,73 \mathrm{ab}$ & $814,96 \pm 51,46 \mathrm{ab}$ & $664,67 \pm 167,84 \mathrm{ab}$ \\
'HG9850' & $422,75 \pm 18,96 \mathrm{a}$ & $695,63 \pm 39,43 \mathrm{ab}$ & $559,19 \pm 152,00 \mathrm{a}$ \\
'HM964'* & $822,83 \pm 28,91 \mathrm{cde}$ & $825,43 \pm 23,56 \mathrm{ab}$ & $824,13 \pm 23,63 \mathrm{abc}$ \\
'Mitger' & $832,44 \pm 28,91 \mathrm{cde}$ & $1509,14 \pm 76,83 \mathrm{de}$ & $1170,79 \pm 374,27 \mathrm{c}$ \\
'SEOP934' & $497,04 \pm 20,18 \mathrm{ab}$ & $738,04 \pm 75,04 \mathrm{ab}$ & $617,54 \pm 140,85 \mathrm{a}$ \\
\hline
\end{tabular}

The donor of PPV resistance' 'Goldrich' and hybrids between 'Goldrich' and the Mediterranean autochthonous varieties 'Ginesta' and 'Palau' (Figure 14) presented more than $50 \%$ of red blush in the skin and the higher amounts of total polyphenol content. The variety "Mitger' contributes as well to the total polyphenol content of hybrids. Results indicated that hybrids from these 3 varieties crossed with 'Goldrich' produced genotypes with interesting polyphenol content. 


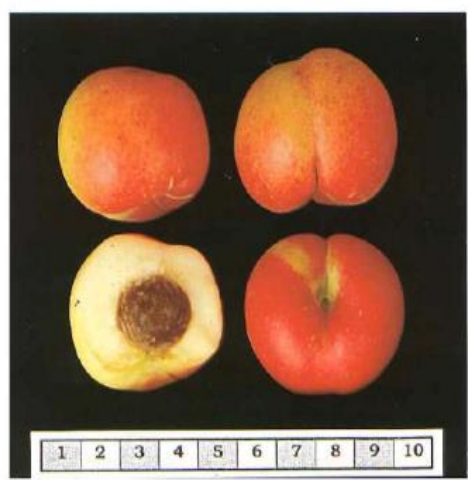

A

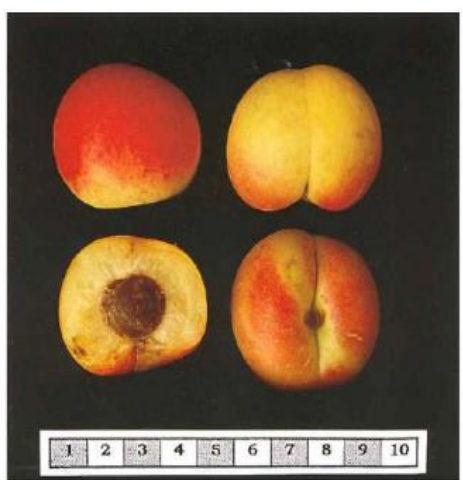

B

Figure 14. Examples of apricot fruits from Mediterranean varieties used as genitors in the breeding program with high redblush on the skin. This trait resulted related to anthocyanin content. A: Fruits from 'Ginesta'; B: Fruits from 'Palau'.

The main secondary phenolic compounds: rutin, quercetin, chlorogenic and neochlorogenic acid were analysed and similar trend was obtained (Figure 15).

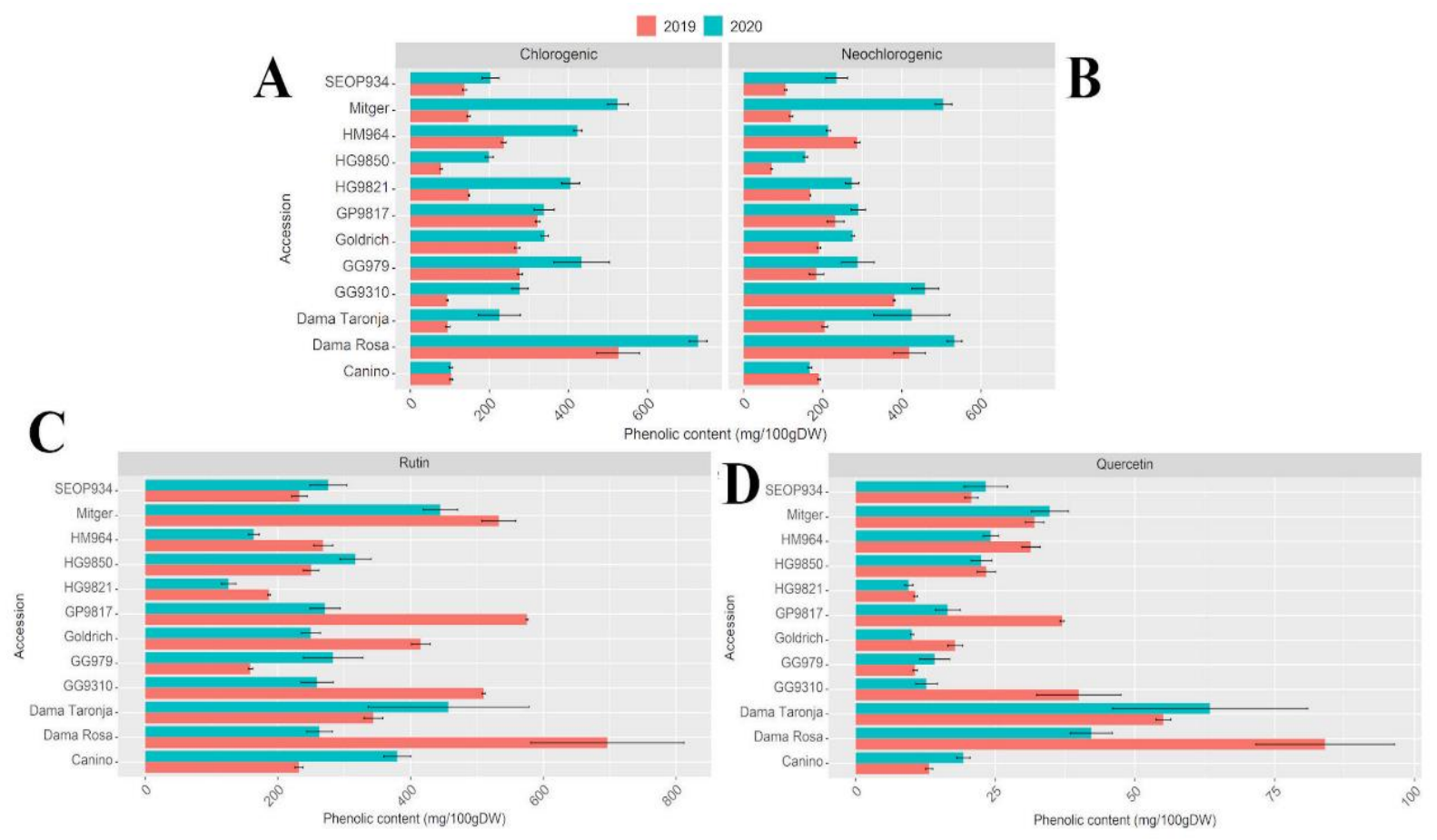

Figure 15. Chlorogenic (A), neochlorogenic (B), rutin (C) and quercetin-3-glucuronide (D) contents (mg/100g DW) in 2019 and 2020. 


\section{IV.4.2. Putative orthologous and phylogenetic analysis}

BLAST analysis using DFR, FLS and $P A L$ sequences from $P$. persica and $A$. thaliana identified a total of 5 genes in P. armeniaca: ParDFR (PARG07267), ParFLS1 (PARG08425), ParFLS2 (PARG08426), ParPAL1 (PARG18722), ParPAL2 (PARG02214). A high level of conservation (>95\%) between peach and apricot genes was observed (Table S12). PAL genes were located in different linkage groups in both species, and as a consequence, in different synteny block. PpePAL1 was in LG2, meanwhile in apricot was in LG5. However, PpePAL2, located in LG6, matched in LG1 in apricot. PpeDFR, PpeFLS1 and PpeFLS2 were located in LG1 in peach, but they match with LG2 in apricot. In addition, $A$. thaliana and apricot also had a high identity (>80\%) for PAL, more than $70 \%$ for ParDFR and $60 \%$ for ParFLS1 and $45.65 \%$ for ParFLS2 (Table S13). In addition, protein alignment also revealed a high conservation among Prunus and $A$. thaliana (Table S14 and S15). ParPAL1 and ParPAL2 showed around 80\% of similarity with AtPAL1 and AtPAL2, respectively. Regarding DFR, similarity was around 70\% mean. FLS showed the lowest similarity with a $57 \%$ and $43 \%$ for FLS1 and FLS2. Similar trend was observed for peach and Arabidopsis.

ParPAL1 and the putative PAL1 orthologous from Prunus persica and Malus domestica, were clustered together. ParPAL2 and its putative orthologous were grouped in a different cluster which showed the differences among both paralogs. The phylogenetic tree of phenylalanine ammonia-lyase proteins (Figure 16A) showed that all Arabidopsis thaliana proteins clustered together.

The phylogenetic tree revealed that DFR proteins of Prunus persica and Prunus armeniaca clustered together, being closed to its orthologous from Malus domestica (Figure 16B).

The predicted proteins encoded by FLS genes of Arabidopsis thaliana grouped in a cluster. On the other hand, Prunus persica predicted proteins from PpeFLS2 and ParFLS2 were grouped in the same cluster, as did Prunus armeniaca PpeFLS1 and ParFLS1. However, Fragaria vesca predicted sequences encoded by FvFLS clustered in another tree branch with the Malus domestica proteins group (Figure 16C). 
A
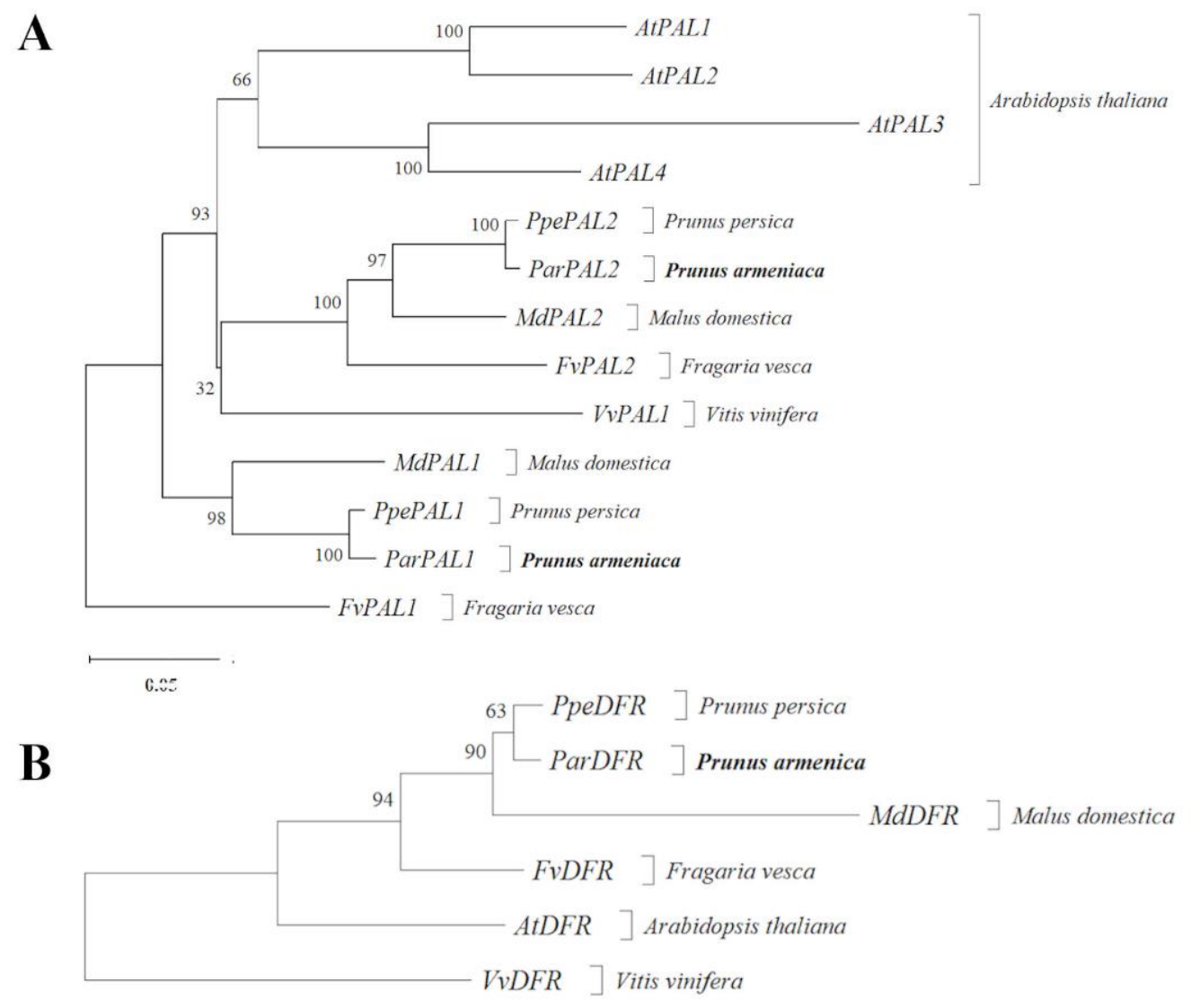

0.10

$\mathrm{C}$

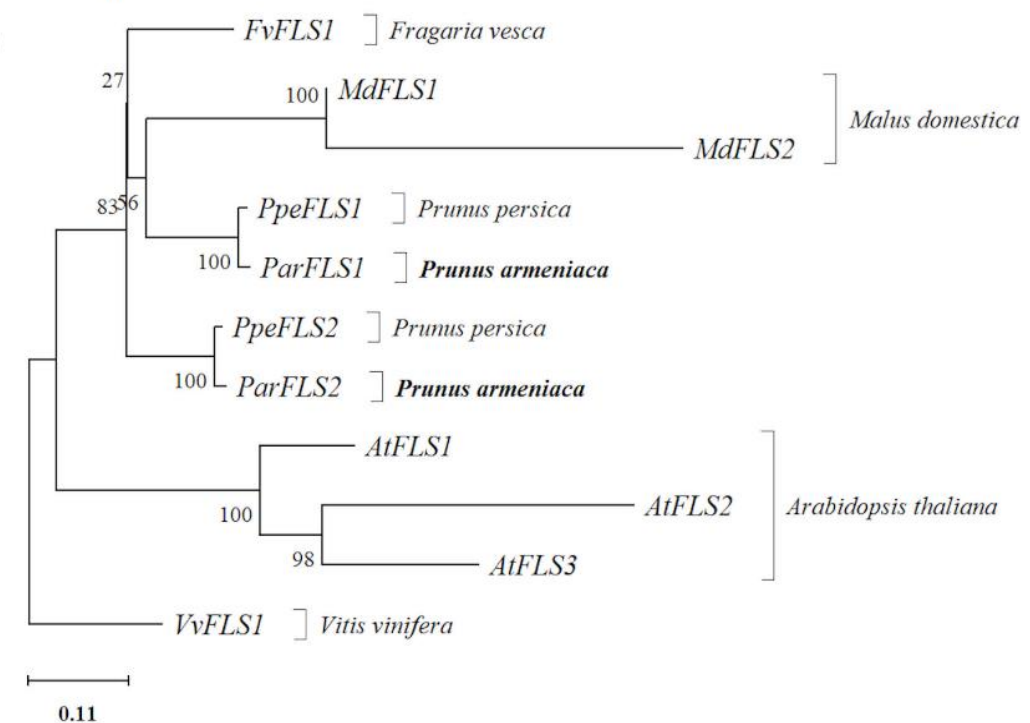

Figure 16. Neighbour-Joining phylogenetic tree for the proteins encoded by PAL (A), DFR (B) and FSL (C) genes. Data was bootstrapped 1000 times. Numbers close to each branch represents the percentage of replicate trees in which the associated taxa clustered together in the bootstrap test. Trees are drawn to scale according to evolutionary distances ( $p$-distance), included under each tree representing the number of substitutions per site. 


\section{IV.4.3. Gene expression}

Genetic expression of the genes studied (ParPAL1, ParPAL2, ParDFR, ParFLS1, ParFLS2) showed no significant differences between years. However, we found minor differences in gene expression among genotypes (Figure 17, Table S16).

Genetic expression of ParPAL1, ParPAL2, ParDFR, and ParFLS2, showed significant differences among genotypes (Figure 17). Concerning to the expression of flavonol-synthase encoding ParFLS1 gene, no significant differences among genotypes were observed.

A
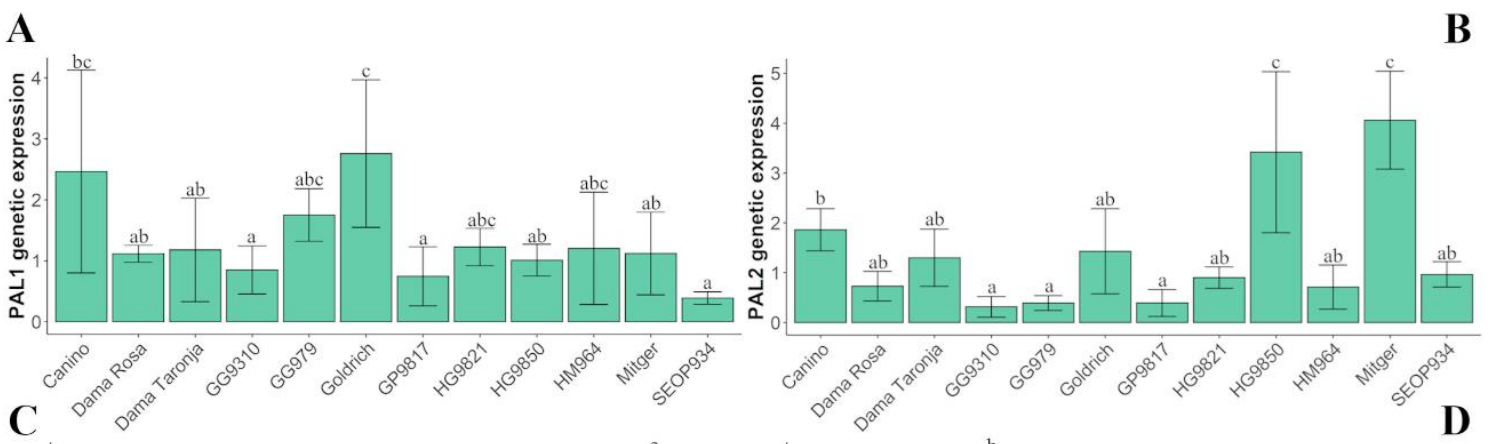

C

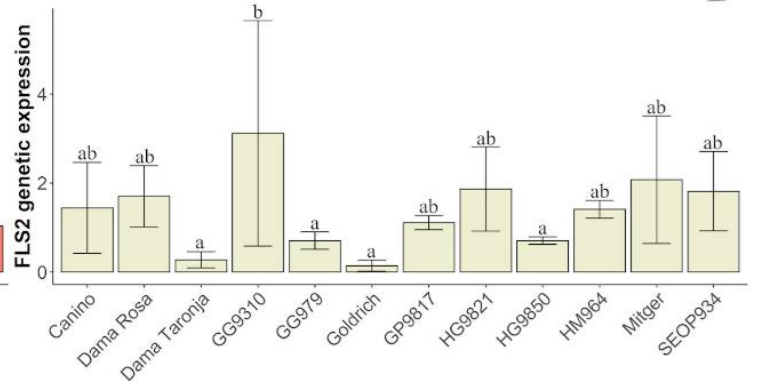

Figure 17. Genetic expression (average of both years of study) of ParPAL1 (A), ParPAL2 (B), ParDFR (C) and ParFLS2 (D). Bars represent standard deviation. Different letters represent statistically significant differences.

Regarding the expression of phenylalanine ammonia-lyase (ParPAL1 and ParPAL2), only the variety 'Goldrich' showed significant differences on PAL1 and two genotypes showed significant differences on PAL2 ('Mitger' and 'HG9850').

\section{IV.4.4. Contribution of 'Goldrich' to phenolic compounds content and genetic expression}

The most important disease affecting Prunus species is caused by the plum pox virus (PPV). In this study 'Goldrich', used as donor of PPV resistance in most apricot breeding programs worldwide and the main contributor to the hybrids included in this study, was evaluated as contributor of compounds for fruit quality (Table 18). 
Table 18. 'Goldrich' contribution to phenolic content: Sum of squares (SS) and model parameters coefficients. SSr: SS relative; SSt: SS total; P-v: P-value; Gr: 'Goldrich' relative; Sig: Significance.

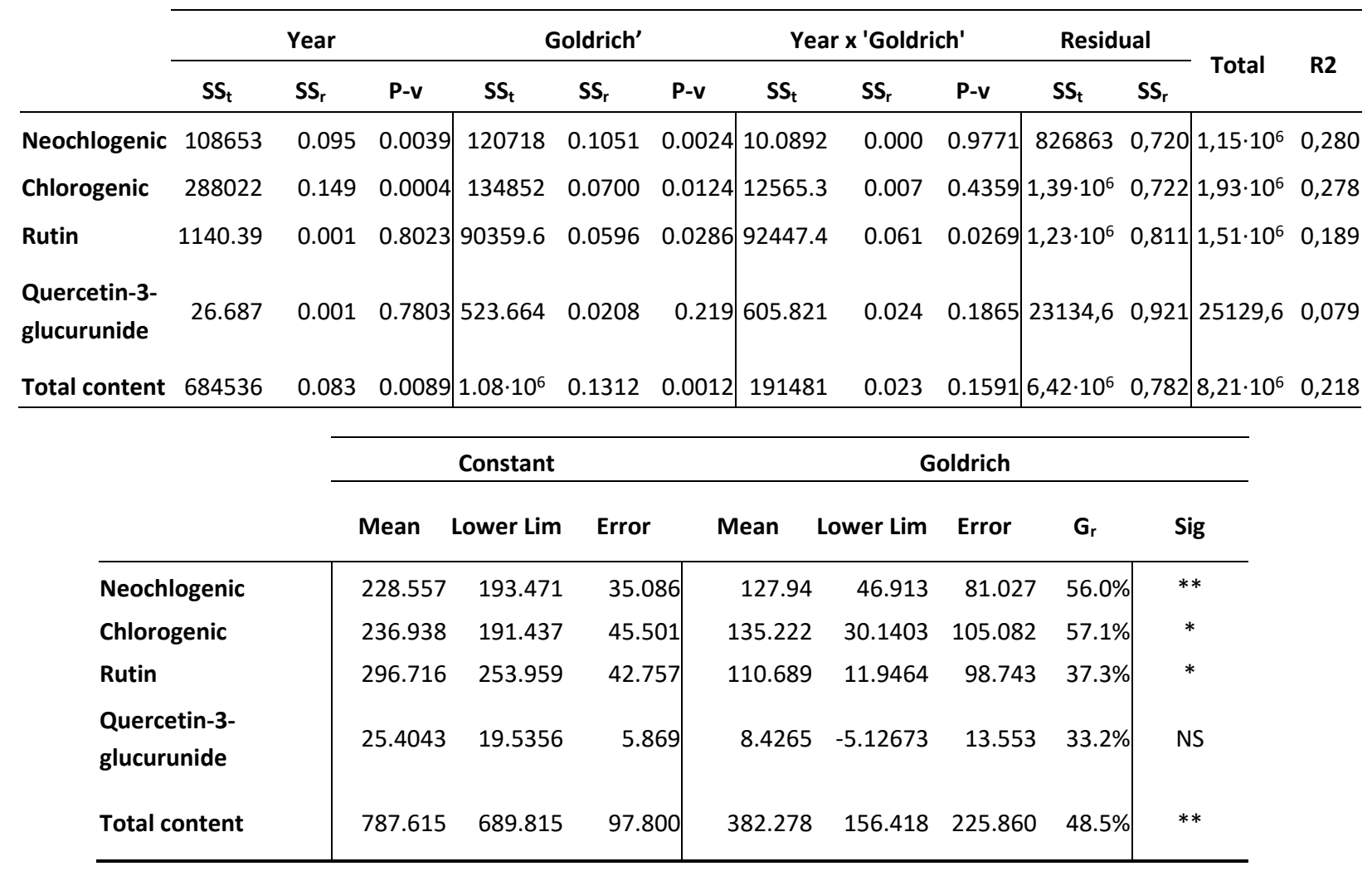

* Significant differences ( $\mathrm{P} \leq 0.05) ; *$ Significant differences $(\mathrm{P} \leq 0.01)$; NS: non-significant

The variety 'Goldrich' showed a significant genetic effect on total polyphenol content. A coefficient of $382.28 \mathrm{mg} / 100 \mathrm{~g}$, which represents more than $45 \%$ of the general average of the population. Similar genetic effect was observed for the specific phenolic compounds, except quercetin-3-glucuronide in which the genetic effect of 'Goldrich' was not significant. The genetic effect of 'Goldrich' for neochlorogenic and chlorogenic acids were 127.94 and $135.22 \mathrm{mg} / 100 \mathrm{~g}$, representing 56\% and 57\% of the general average, respectively. For rutin, the coefficient was $110.7 \mathrm{mg} / 100 \mathrm{~g}$ (37.3\% of the general average).

Concerning genetic expression, the cultivar 'Goldrich' had a significant genetic effect in the expression of all the 5 genes studied: ParPAL1, ParPAL2 ParDFR, ParFLS1 and ParFLS2 (Table 19). The genetic effect of 'Goldrich' varies from $58.2 \%$ in ParFLS2 to $98.7 \%$ in ParDFR. 
Table 19. 'Goldrich' contribution to genetic expression: Sum of squares and model parameters coefficients. SSr: SS relative; SSt: SS total; Pv: P-value; Gr: 'Goldrich' relative; Sig: Significance.

\begin{tabular}{|c|c|c|c|c|c|c|c|c|c|c|c|c|c|}
\hline & \multicolumn{3}{|c|}{ Year } & \multicolumn{3}{|c|}{ Goldrich } & \multicolumn{3}{|c|}{ Year x 'Goldrich' } & \multicolumn{2}{|c|}{ Residual } & \multirow{2}{*}{ Total } & \multirow{2}{*}{ R2 } \\
\hline & $\mathbf{S S}_{\mathrm{t}}$ & $\mathbf{S S}_{\mathrm{r}}$ & P-v & $\mathrm{SS}_{\mathrm{t}}$ & $\mathrm{SS}_{\mathrm{r}}$ & P-v & $\mathrm{SS}_{\mathrm{t}}$ & SS $_{r}$ & P-v & $\mathbf{S S}_{\mathrm{t}}$ & $\mathrm{SS}_{\mathrm{r}}$ & & \\
\hline ParDFR & 2.2294 & 0.023 & 0.1686 & 17.738 & 0.1795 & 0.0002 & 2.4002 & 0.024 & 0.1534 & 78.2977 & 0.792 & 98.8062 & 0,208 \\
\hline ParFLS1 & 17.938 & 0.073 & 0.002 & 8.0677 & 0.0327 & 0.0362 & 11.5896 & 0.047 & 0.0124 & 219.4 & 0.888 & 246.939 & 0,112 \\
\hline ParFLS2 & 0.0474 & 0.000 & 0.8526 & 6.7223 & 0.0636 & 0.0297 & 2.8529 & 0.027 & 0.1527 & 92.7271 & 0.878 & 105.665 & 0,122 \\
\hline ParPAL1 & 0.3709 & 0.006 & 0.5249 & 4.2923 & 0.0655 & 0.0332 & 0.3443 & 0.005 & 0.5401 & 60.8265 & 0.928 & 65.5523 & 0,072 \\
\hline ParPAL2 & 1.0437 & 0.009 & 0.4138 & 16.0300 & 0.1310 & 0.0020 & 0.1313 & 0.001 & 0.7714 & 104.974 & 0.859 & 122.217 & 0,141 \\
\hline
\end{tabular}

\begin{tabular}{|c|c|c|c|c|c|c|c|c|}
\hline & \multicolumn{3}{|c|}{ Constant } & \multicolumn{5}{|c|}{ Goldrich } \\
\hline & Mean & Lower Lim & Error & Mean & $\begin{array}{c}\text { Lower } \\
\text { Lim }\end{array}$ & Error & $\mathbf{G}_{\mathbf{r}}$ & Sig \\
\hline ParDFR & 1.57183 & 1.2304 & 0.341 & -1.5509 & -2.3393 & 0.788 & $-98.7 \%$ & $* *$ \\
\hline ParFLS1 & 1.54347 & 1.2259 & 0.318 & -0.7697 & -1.4891 & 0.719 & $-49.9 \%$ & $*$ \\
\hline ParFLS2 & 1.64119 & 1.2696 & 0.372 & -0.9547 & -1.8128 & 0.858 & $-58.2 \%$ & $*$ \\
\hline ParPAL1 & 1.08334 & 0.7799 & 0.303 & 0.7652 & 0.0628 & 0.702 & $70.6 \%$ & $*$ \\
\hline ParPAL2 & 1.80279 & 1.4075 & 0.395 & -1.4731 & -2.3861 & 0.913 & $-81.7 \%$ & $* *$ \\
\hline
\end{tabular}

* Significant differences ( $\mathrm{P} \leq 0.05)$; * Significant differences ( $\mathrm{P} \leq 0.01)$; NS: non-significant

IV.4.5. Relationships between gene expression and phenolic compound accumulation.

A correlation analysis performed among compounds and expression of genes studied revealed a significant correlation between neochlorogenic acid and the rest of phenolic compounds. (Table 20).

Table 20. Pearson correlation coefficients among compounds and gene expression.

\begin{tabular}{lccccccc}
\hline Parameter & ParPAL1 & ParPAL2 & ParDFR & ParFLS1 & ParFLS2 Neochlorogenic Chlorogenic & Rutin \\
\hline ParPAL1 & & & & & & & \\
ParPAL2 & 0.1507 & & & & & & \\
ParDFR & -0.0163 & $0.8098^{* *}$ & & & & & \\
ParFLS1 & -0.1899 & $0.3408^{* *}$ & $0.3139^{* *}$ & & & \\
ParFLS2 & $-0.2726^{*}$ & 0.0273 & 0.1629 & 0.1261 & & \\
Neochlorogenic & -0.1951 & -0.1369 & -0.0258 & -0.1043 & $0.2919^{*}$ & & \\
Chlorogenic & -0.1317 & -0.1216 & -0.017 & 0.0051 & -0.011 & $0.6835^{* *}$ & \\
Rutin & 0.0283 & 0.1635 & 0.0568 & -0.2062 & 0.1943 & $0.2929^{*}$ & 0.0734 \\
Quercetin-3- & -0.1638 & 0.0083 & 0.0477 & -0.144 & 0.1216 & $0.4452^{* *}$ & 0.2233 \\
glucuronide & & & & & & \\
\hline
\end{tabular}

* Significant differences $(P \leq 0.05) ; * *$ Significant differences $(P \leq 0.01)$. 
ParDFR expression revealed a positive correlation with ParAL2 (0.8) but also showed positive correlation with ParFLS1, which also correlated positively with ParPAL2. The gene expression obtained indicates interaction among the genes selected in key steps of the polyphenol pathway.

To complete the previous study, we studied the relationships between the gene expression and each phenolic compound content through a linear regression model (Tables S17 and S18). The trend between the phenolic compounds content and the expression of genes obtained is summarized in Figure 18.

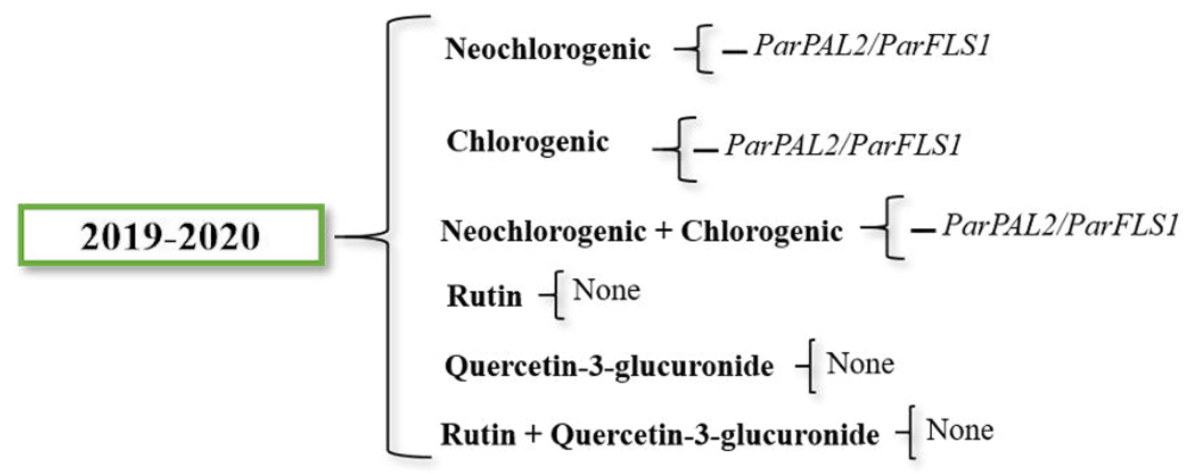

Figure 18. Significant correlations from the linear regression model for each compound.

Both neochlorogenic and chlorogenic acid content were negatively influenced by ParPAL2/ParFLS2 ratio. Due to neochlorogenic and chlorogenic acids are synthetized in the same pathway branch, the correlation between their content and the gene expression was evaluated also together. Data from the two-years average revealed a negative impact of ParPAL2/ParFLS1 in the neochlorogenic and chlorogenic total content. For rutin and quercetin-3-glucuronide content, no significant correlation was found. The gene expression effect was low in the levels of accumulation of all the compounds.

\section{IV.4.6. Cis-acting elements analysis}

Due to the correlation among expression of some genes, a study of upstream sequences to find cisacting elements recognized by MYB-like transcription factors was carried out (Figure 19). 

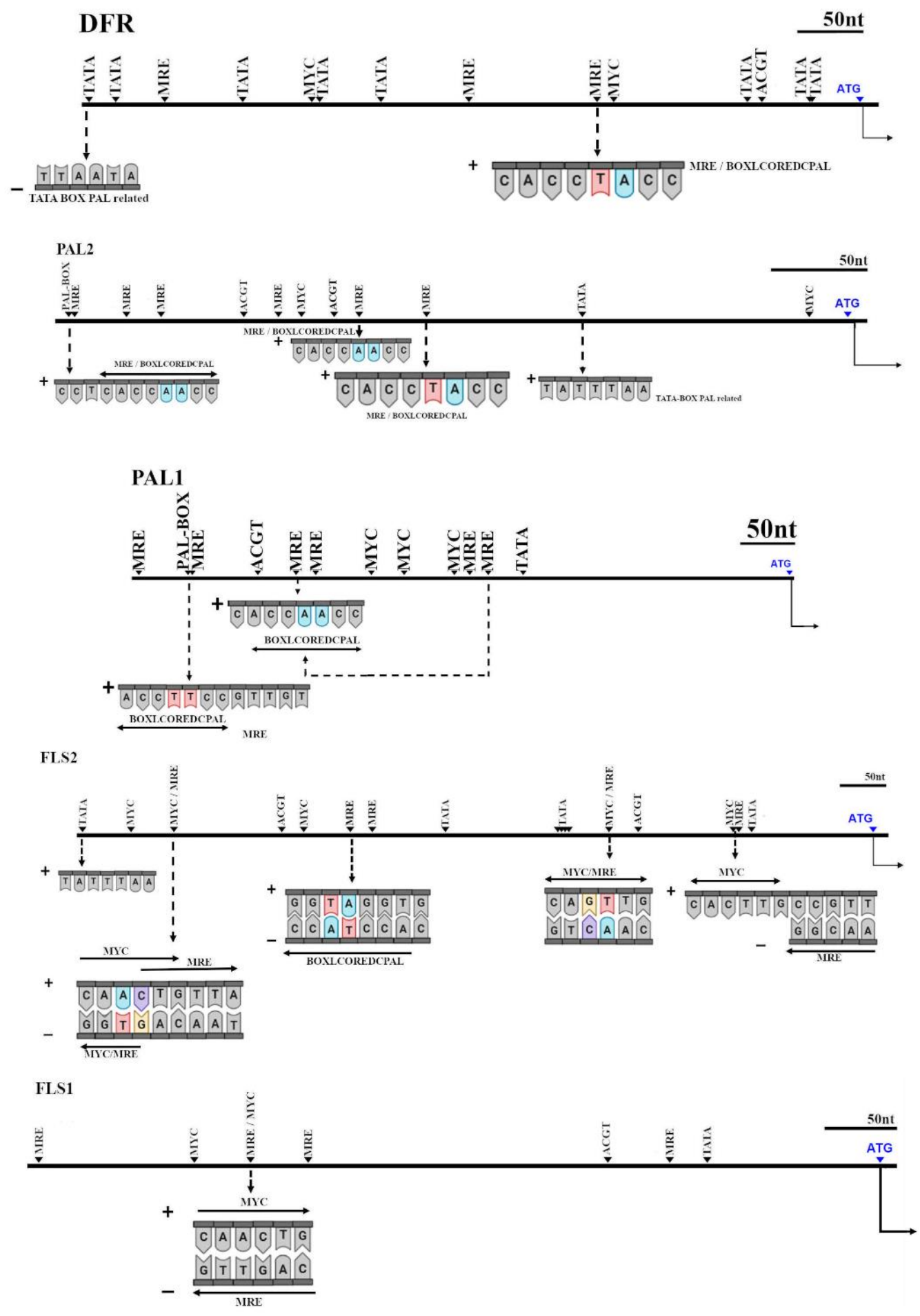

Figure 19. Analysis of cis-acting elements on 1500bp upstream from start codon (ATG) sequences of Prunus armeniaca DFR, PAL and FLS genes. MRE: MYB-like Recognition Element; MYC: MYC-like recognition sequence; TATA: TATA box-like; BOXLCOREDCPAL: Consensus of the putative "core" sequences of box-L-like PAL promoter region. 
In ParDFR, we found at 694bp upstream from ATG, a TATA-BOX-PAL related, next to other TATA-boxlike motif and MRE (a MYB-recognition element). In addition, a MYC motif was found together with a TATA-box-like. Besides, at 238 bp upstream from ATG, a MRE was found encoding also a BOXLCOREDCPAL, a motif related with the PAL promoter region. This MRE was closed to a MYC motif. In ParPAL2, 403 bp and 255 bp upstream from ATG we found a MRE encoding a BOXLCOREDCPAL, with a different sequence from the one found in ParDFR. However, 220bp upstream from ATG we found the same MRE encoding a BOXLCOREDCPAL as found in DFR. Besides, a TATA-BOX-PAL related was found 139 bp upstream.

However, in ParPAL1 we did not find the same MRE encoding the BOXLCOREDCPAL, found in ParDRF and PAL2 upstream. Indeed, we found 551bp upstream from ATG, also the same MRE motif but differing only in a nucleotide. On the other hand, 276bp upstream we found a MRE encoding a PALbox-like motif, identical as found twice in PAL2.

In ParFLS1 we found four MRE, but none of them encoded a PAL-box-like motif. However, 438bp upstream from ATG, we found a MYC motif, but also a MRE antisense.

In ParFLS2, we found 572 bp upstream the same MRE encoding a BOXLCOREDCPAL, as found in ParDFR and ParPAL2. Furthermore, 765bp upstream we found the same MYC/MRE motif found in ParFLS1. Moreover, the same cis-acting element was found antisense 289 bp upstream from ATG, but antisense.

\section{IV.5. Discussion}

\section{IV.5.1. Polyphenol content}

Total polyphenol and individual phenolic compounds analysed were genotype-dependent. The higher values corresponded to genotypes derived from varieties characterized by important red skin colour, as the Mediterranean autochthonous varieties 'Ginesta', 'Palau' and 'Mitger' or the donor of resistance to PPV 'Goldrich'. This fact agrees with the references in which polyphenol content, anthocyanins and red colour of fruits are related (Dossett et al., 2011; Kayesh et al., 2013). On the other hand, the linear model indicates that contribution of the variety 'Goldrich' to the content of polyphenols is remarkable in agreement with previous results (Gómez-Martínez et al., 2021). This suggest that the introgression of resistance to PPV (the most important objective of the apricot breeding programs worldwide) is not negatively affecting the fruit quality of apricot, another important objective of the apricot breeding programs from the Mediterranean basin.

Genetic expression of ParPAL1 was relevant in those accessions that showed high content in phenolic compounds as 'Goldrich'. Indeed, phenylalanine ammonia-lyase (PAL) plays a significant role in the phenylpropanoid metabolism pathway. PAL, as the first key enzyme in phenylpropanoid biosynthesis, 
catalyses the conversion of L-phenylalanine to cinnamic acid, linking primary metabolism with secondary metabolism and becoming a speed-limiting step in phenylpropanoid metabolism (Wang et al., 2014b). In Prunus species, this genetic family consists of two PAL members (Irisarri et al., 2016) and in our study they were identified in apricot by synteny with peach (ParPAL1 and ParPAL2). According to our results, ParPAL1 activity contributes to peel phenolic accumulation in the group of genotypes studied.

The next critical step analysed is the one where the phenolic pathway branches off towards anthocyanins or flavonol synthesis. Dihydroflavonol reductase (DFR) is an enzyme that catalyses the reduction from dihydroflavonols to anthocyanins biosynthesis (Lo Piero et al., 2006; Martens et al., 2003; Shimada et al., 2005). Our results revealed major ParDFR expression in hybrids from cultivars with high percentages of red blush, like the ones described by Badenes et al. (2018). This red coloration could be associated with anthocyanin accumulation as showed previous studies in apricot (Bureau et al., 2009). Consequently, our results may suggest a higher ParDFR expression in those cultivars with high percentages of red blush on the fruit skin.

Alternatively, flavonol synthase (FLS) catalyses the reaction from dihydroflavonols to flavanols, a group of flavonoids in which rutin and quercetin-3-glucuronide are found. In apricot, two FLS encoding genes are present: ParFLS1 and ParFLS2. Two crop years average revealed lower expression of ParFLS2 in those genotypes without contribution of autochthonous genitors characterized by redblush fruits. High expression was obtained in hybrids from cultivars with an important percentage ( $>50 \%$ ) of fruit skin covered by a red blush with a high intensity of over colour (Badenes et al., 2018). Additionally, most of the cultivars of this group were also reported as the accessions with major total content in polyphenols. These results are in agreement with previous works indicating that expression of $F L S$ could be related to phenolic biosynthesis and also linked with anthocyanins accumulation (Kuhn et al., 2011; Owens et al., 2008).

At gene expression level, 'Goldrich' effect was correlated positively with ParPAL1. Taking into account that 'Goldrich' has a positive contribution to polyphenol content, this fact suggests that ParPAL1 expression levels are related to the accumulation of phenolic compounds.

IV.5.2. Genes and its inference in polyphenols pathway

Both PAL and FLS putative orthologous analysis resulted in two genes per enzyme identified in $P$. armeniaca genome. Genome duplication is common among plants, leading to the duplication of genes (Roulin et al., 2013). Indeed, it has been described that Rosaceae family origin comes from a polyploidization event, explaining the presence of two of these genes in the Rosaceae species (Xiang et al., 2017). In agreement, $A$. thaliana presents three copies of $F L S$ and four of $P A L$, as result of the 
two polyploidization events that originated this species (Bomblies and Madlung, 2014; Bomblies and Weigel, 2007). Functional redundancy and natural selection lead to gene loss, silencing or neofunctionalized (del Pozo and Ramirez-Parra, 2015). Dosage-dependent genes are usually retained in the duplicated genomes (Edger and Pires, 2009), suggesting the dosage dependence of $F L S$ and PAL in the phenylpropanoid pathway.

Previous studies related MYB transcription factors with phenolic biosynthesis in various species (Jin et al., 2016; Luo et al., 2016). In fact, Hartmann et al. (2005) showed a relation between cis-acting elements recognized by R2R3-MYB (or MYB-recognition element (MRE)), BZIP (ACGT-element), and BHLH (CANNTG motif) with phenylpropanoid biosynthesis genes.

Taking into account this information, we did a screening of possible MRE cis-acting elements involved in phenolic biosynthesis. Results revealed a common MRE (MYBCORE) containing also a BOXLCOREDCPAL motif in ParDFR and ParPAL2, which suggested that both genes can be regulated by the same transcription factor. However, this MRE was not found in ParPAL1. This fact suggests different regulation or even different roles of each identified PAL paralogues in apricot. This is also supported by the high correlation of ParDFR and ParPAL2 expression, which indicates that they share the same regulation and supports the existence of different regulation for each paralog. This specialization between paralogous that result from ancestral genomic duplications has been previously described (Lian et al., 2020) and even can lead to neo-functionalization of genes. In addition, most of the accessions with a high expression for ParFLS2, such as "Dama Rosa', are siblings of the traditional cultivar 'Ginesta', a cultivar that had more than $50 \%$ of red blush (Badenes et al., 2018). These results suggest a possible role of ParFLS2 in anthocyanin synthesis, in agreement with previous studies that proposed a disequilibrium in the expression of $F L S$ and DFR enzymes determine the accumulation of flavonols and anthocyanins (Kuhn et al., 2011; Luo et al., 2016; Owens et al., 2008).

As the transcriptomic study was made at fruit maturity, a further analysis of ParPAL1 in different immature fruit stages would contribute to identify accurately its role in peel polyphenol content. Furthermore, the results obtained indicated a possible shared regulation for ParFLS2 and ParDFR expression related to anthocyanin biosynthesis in apricot. Our results contribute to unravel the relationship between genetic of red-blush trait and polyphenol compounds and the relationship between ParFLS2 and anthocyanin biosynthesis in apricot.

\section{IV.6. Conclusions}

The set of accessions studied showed that polyphenols content is genotype-dependant. In addition, cultivar 'Goldrich', used as donor of PPV resistance contributed positively to phenolic content and ParPAL1 expression. Transcriptomic data of the main genes involved in critical points at the polyphenol 
pathway have been described and its relationships with the different polyphenol compounds identified. Higher expression of ParDFR and ParPAL2 has been associated to red-blushed accessions. Differences in expression between paralogues in the phenolic pathway can be linked to the presence of a BOXCOREDLPAL cis-acting element related to the genes involved in anthocyanin synthesis: ParDFR, ParFLS2, and ParPAL2. 
GENERAL DISCUSSION 
Nowadays there is an increasing demand for safe, healthy, and nutritious food by consumers, turning the internal quality of the fruit into one of the main goals of breeding programs. Plants and more specifically some biocompounds of fruits are an excellent source of compounds with health benefits (Slavin and Lloyd, 2012; Vieira da Silva et al., 2016). From a dietary point of view, apricot is an excellent fruit that has an important role in human nutrition and it is an excellent source of sugars, fibres, vitamins, organic acids, and antioxidants (Sochor et al., 2010). However, must be taken into consideration that pomological and nutraceutical fruits properties vary with cultivation systems, environmental condition, fruit storage and fruit developmental stage (Bae et al., 2014; Drogoudi et al., 2008; Ruiz et al., 2005).

Apricot has very specific ecological requirements and each region usually grows locally adapted cultivars. For this reason, significant breeding efforts have been undertaken worldwide (Zhebentyayeva et al., 2012). The apricot breeding program at the Instituto Valenciano de Investigaciones Agrarias (IVIA) started in 1993 with the main purpose of obtaining new varieties resistant to the Plum Pox Virus (PPV), but also self-compatibles, with high fruit quality, and welladapted to the Southern European environment (Martínez-Calvo et al., 2009). At this moment, the emergence of the sharka disease forced the use of varieties not adapted to our conditions, like 'Goldrich', as donors of resistance. At present, numerous resistant varieties have been developed in the ongoing programs worldwide using these same sources (Polo-Oltra et al., 2020). After a great effort in gene mapping, transcriptomic and genomic data pointed out silencing of the ParPMC genes as the responsible for the PPV resistance in apricot (Zuriaga et al., 2018). Following a similar strategy, the development of new varieties with higher levels of nutritional attributes and also the study of the genetic control of these traits have been included as a new goal at the IVIA's apricot breeding program. For this purpose, as the first step of any breeding program, in this thesis part of the IVIA's germplasm collection was screened in order to identify sources of variation for the traits of interest. Results allowed the identification of parental lines with interesting nutraceutic profiles, that could be attractive for the breeding program goals concerning to main phenolic compounds, ascorbic acid, sugars, and organic acids.

The presence of high levels of nutraceutical compounds could be interesting for fresh consumption but also for the food industry. In this sense, the analysis of these compounds in different tissues could be interesting. In agreement with previous studies, our results showed a high peel content among the different accessions studied for all the analyzed compounds. For this reason, apricot peel could be pointed as a potential source of bioactive compounds for developing new functional foods or food additives, as occurs with others fruit wastes (Deng et al., 2012; Kumar et al., 2017).

Nutraceutical profiles of the analyzed IVIA's accessions showed variability for all the studied 
compounds. According to our analysis of sugar content, sucrose, followed by glucose, fructose and sorbitol have been identified as the main sugars in apricot, in agreement with results reported by other authors, like Cirilli et al. (2016) and Karabulut et al. (2018). However, the consumer perception of sweetness intensity depends on the quantity but also the proportions between the different sugars. In this sense, some accessions have been identified as potential candidates as sweetness sources. Organic acids also have an important role in apricot taste (Xi et al., 2016). Citric and malic acids were identified as the major ones in apricot as also occurs in other fruits. Some of the analyzed accessions showed an optimal malic:citric ratio according to Dolenc- Šturm et al. (1999) making them good candidates for fresh consumption. Additionally, the organic acids content of some accessions pointed them as useful for the food-industry. Ascorbic acid content showed significant differences between genotypes but also years. However, 'HM964' showed a high content but also a stable behaviour that points it as a promising pre-breeding material. Finally, 'Goldrich' showed the highest content of polyphenols, both phenolic acids and flavonoids, among the studied accessions. Interestingly, the range of values observed confirmed that apricot is a good source of phytochemicals with antioxidant potential as suggested by (Fan et al., 2018).

The genetic control of these traits is complex and also very influenced by the environmental conditions, making it more difficult to study. The efficiency of breeding programs could increase dramatically if genetic information is available, especially in the case of trees and/or fruit-related traits (Illa et al., 2011). Despite the enormous advances in genomics in recent years, there is still much to know about the genetic control of these traits and to be able to apply marker-assisted selection to help and improve the efficiency of the breeding programs. Expression of fruit-quality related candidate genes was evaluated taking into account that some QTLs linked to fruit quality traits have been described in apricot (Salazar et al., 2013 and 2017; García-Gómez et al., 2021) and peach (Cirilli et al., 2016), and the high synteny level between Prunus spp (Arús et al., 2010; Campoy et al., 2011). Phylogenetic analysis of the studied genes in different species showed a high level of conservation and confirmed the high level of synteny among Prunus spp. (Arús et al., 2010; Campoy et al., 2011; Dirlewanger et al., 2004). Regarding the sugar-related gene expression, non-significant differences between genotypes were observed, although it was observed for sugar contents. Further analyses should be necessary to analyse in detail the role of these genes in the control of the trait in apricot. Regarding polyphenolrelated genes, BLASTP analysis identified 5 apricot genes related to related to primary phenolic compounds (phenylalanine ammonia-lyases, PAL), anthocyanin biosynthesis (dihydroflavonol-4reductase, DFR) and secondary phenolic metabolites (flavonol synthases, FLS). The accessions showing a red blush in the fruit skin showed a higher expression of ParDFR and ParPAL2. The presence of MYBlike cis-acting elements has been related with phenolic biosynthesis (Hartmann et al., 2005). The 
screening of the presence of putative MRE cis-acting elements upstream of these genes revealed a BOXLCOREDCPAK motif in PARDFR and ParPAL2 that could indicate a common regulation pattern. These preliminary studies will be completed with new analyses at different fruit development stages in order to confirm these results.

'Goldrich' is a North-American well-known apricot cultivar, obtained in 1954 by the Washington State Agricultural Service (USA) (Brooks and OImo, 1997). It is one of the few available PPV resistance sources and has been commonly used as a donor in all the apricot breeding programs currently in progress (Martínez-Gómez et al., 2000). Indeed, 'Goldrich' is the female parent of 'Dama Taronja' or 'Dama Rosa', two mid-early ripening apricot cultivars obtained at the IVIA's breeding program (Badenes et al., 2018). The study of the genetic contribution of 'Goldrich' to the phenolic content of the offspring using an adapted model from Gómez and Ligarreto (2012), showed that in addition to the PPV resistance also improves fruit quality in terms of phenolic content.

In conclusion, the IVIA's apricot germplasm collection preserves good variability in terms of fruit quality attributes that may be of interest for the improvement of the species. Results of this thesis facilitate the selection of parental lines for apricot breeding but also for the development of progenies that will be useful to study the genetic control analysis of these traits. 
GENERAL CONCLUSIONS 
- Apricot is a fruit crop very appreciated by consumers due to its organoleptic characteristics. The results presented here also confirm this fruit species as an important source of phytocompounds as sugars, organic acids, vitamins, and polyphenols.

- The presence of high content in apricot peel points this tissue as an excellent source of nutraceutical compounds.

- Sucrose and glucose were identified as the major sugars in apricot, meanwhile malic and citric acid were the main organic acids, and ascorbic acid content varied among the studied cultivars.

- Some potentially interesting accessions for fresh consumption due to their sweetness intensity or the optimal ratio between organics acids have been identified in the germplasm collection, but also others that could be interesting for the food industry as sources of malic acid.

- The high synteny between Prunus spp. is useful to study candidate genes in less studied species, such as apricot. Sugar-related gene sequences from other species have been used to identify putative orthologous in apricot. The genetic expression of a sorbitol dehydrogenase (ParSDH), a fructokinase (ParFK1), three sucrose-synthases (ParSUS1, ParSUS3 and ParSUS6), and three sucrose-phosphate-synthases (ParSPS1, ParSPS2 and ParSPS3)) showed differences between genotypes in some cases.

- In some cases a correlation between metabolite content and the expression of the related genes analyzed were observed, such as a high sucrose content and the expression of genes related to sucrose synthesis in 'Canino', but not in all cases.

- The set of apricot accessions analysed showed different content in the polyphenols compounds analyzed, but this content is environmental dependent.

- The cultivar 'Goldrich', used worldwide as a donor of resistance to sharka disease, was the highest contributor to the polyphenol content among the accessions studied. The genetic effect of 'Goldrich' in this trait indicated it was a good candidate for increasing both neochlorogenic and chlorogenic acid content of fruits in the IVIA's breeding program.

- Higher expression of ParDFR and ParPAL2 has been associated with red-blushed accessions.

- Differences in expression between paralogous in the phenolic pathway could be linked to the presence of a BOXCOREDLPAL cis-acting element observed in ParDFR, ParFLS2, and ParPAL2 genes, involved in anthocyanin synthesis.

- Sources of variation for the content of sugars, organic acids, ascorbic acid, and phenolic compounds have been identified at the IVIA's apricot germplasm collection. These results pave the way for the incorporation of these traits as breeding goals but also for future studies to elucidate the genetic control of these traits. 
REFERENCES 
Abbott, A.G., Arús, P., and Scorza, R. (2008). Genetic engineering and genomics, in: The Peach: Botany, Production and Uses. (CABI Publishing), 85-105. ISBN: 9781845933869 https://doi.org/10.1079/9781845933869.0085

Agustí Fonfría, M. (2010). Fruticultura. (España: Mundi-Prensa Libros). ISBN: 9788484765295.

Akin, E.B., Karabulut, I., and Topcu, A. (2008). Some compositional properties of main Malatya apricot (Prunus armeniaca L.) varieties. Food Chem., 107, 939-948. https://doi.org/10.1016/J.FOODCHEM.2007.08.052

Alioto, T., Alexiou, K.G., Bardil, A., Barteri, F., Castanera, R., Cruz, F., et al. (2020). Transposons played a major role in the diversification between the closely related almond and peach genomes: results from the almond genome sequence. Plant J., 101, 455-472. https://doi.org/10.1111/tpj.14538

Almeida, M.M.B., de Sousa, P.H.M., Arriaga, Â.M.C., do Prado, G.M., Magalhães, C.E.C., Maia, G.A., et al. (2011). Bioactive compounds and antioxidant activity of fresh exotic fruits from northeastern Brazil. Food Res. Int., 44, 2155-2159. https://doi.org/10.1016/j.foodres.2011.03.051

Andre, C.M., Ghislain, M., Bertin, P., Oufir, M., Herrera, M.D.R., Hoffmann, L. et al. (2007). Andean potato cultivars (Solanum tuberosum L.) as a source of antioxidant and mineral micronutrients. $J$. Agric. Food Chem., 55, 366-378. https://doi.org/10.1021/jf062740i

Arts, I.C.W., Van De Putte, B., and Hollman, P.C.H. (2000). Catechin contents of foods commonly consumed in The Netherlands. 1. Fruits, vegetables, staple foods, and processed foods. J. Agric. Food Chem., 48, 1746-1751. https://doi.org/10.1021/jf000025h

Arús, P., Yamamoto, T., Dirlewanger, E., and Abbott, A.G. (2010). Synteny in the Rosaceae. Plant Breed. Rev., 27, 175-211. https://doi.org/10.1002/9780470650349.CH4

Audergon, J.M., Blanc, A., Gilles, F., Clauzel, G., Broquaire, J. M., Gouble, B., et al. (2011). An integrated apricot breeding program in france joining CEP innovation - CENTREX and INRA. International Symposium on Apricot Breeding and Culture. Acta Hortic., 966, 17-21. https://doi.org/10.17660/ActaHortic.2012.966.1

Audergon, J.M., Blanc, A., Gilles, F., Gouble, B., Grotte, M., Reich, M., et al. (2009). New recent selections issued from INRA's apricot breeding program. Acta Hortic., 814, 221-226. https://doi.org/10.17660/ActaHortic.2009.814.30 
Azmir, J., Zaidul, I.S.M., Rahman, M.M., Sharif, K.M., Mohamed, A., Sahena, F., et al. (2013). Techniques for extraction of bioactive compounds from plant materials: A review. J. Food Eng., 117, 426-436. https://doi.org/10.1016/J.JFOODENG.2013.01.014

Badenes, M.L., and Hutagalung, A. (2012). Fruit breeding. (Boston (MA, US): Springer). https://doi.org/10.1007/978-1-4419-0763-9

Badenes, M.L., Martínez-Calvo, J., Gómez, H., and Zuriaga, E. (2018). 'Dama Taronja' and 'Dama Rosa' apricot cultivars that are resistant to sharka (Plum pox virus). HortScience, 53, 1228-1229. https://doi.org/10.21273/HORTSCI13155-18

Badenes, M.L., Moustafa, T.A., Martínez-Calvo, J., and Llácer, G. (2006). Resistance to sharka trait in a family from selfpollination of "Lito" apricot cultivar. Acta Hortic., 381-384. https://doi.org/10.17660/ActaHortic.2006.701.63

Badenes, M. L., and Zuriaga, E. (2016). “Las variedades locales en la mejora genética de plantas”, in Las variedades locales en la mejora genética de plantas, eds. J.I. Ruiz de Galarreta, J. Prohens, and R. Tierno (Vitoria-Gasteiz, Spain: Gobierno Vasco), 239-259. ISBN: 978-84-457-3395-0

Bae, H., Yun, S.K., Jun, J.H., Yoon, I.K., Nam, E.Y., and Kwon, J.H. (2014). Assessment of organic acid and sugar composition in apricot, plumcot, plum, and peach during fruit development. J. Appl. Bot. Food Qual., 87, 24-29. https://doi.org/10.5073/JABFQ.2014.087.004

Bailey, C.H., and Hough, L.F. (1975). "Apricots”, in Advances in fruit breeding, eds. J. Janick and J. Moore (West Lafayette, IN (USA): Purdue University Press), 367-383. ISBN: 0911198369

Bassi, D., and Audergon, J.M. (2006). Apricot breeding: update and perspectives. Acta Hortic., 701 I, 279-294. https://doi.org/10.17660/ACTAHORTIC.2006.701.43

Bassi, D., Bartolozzi, F., and Muzzi, E. (1996). Patterns and heritability of carboxylic acids and soluble sugars in fruits of apricot (Prunus armeniaca L.). Plant Breed., 115, 67-70. https://doi.org/10.1111/j.1439-0523.1996.tb00873.x

Bassi, D., Bellini, E., Guerriero, R., Monastra, F., and Pennone, F. (1995). Apricot breeding in Italy. Acta Hortic., 384, 47-54. https://doi.org/10.17660/ActaHortic.1995.384.3

Bassi, D., Rizzo, M., and Foschi, S. (2010). Breeding apricot in Northern Italy. Acta Hortic. 862, 151-158. https://doi.org/10.17660/actahortic.2010.862.23

Bassi, D., and Selli, R. (1990). Evaluation of fruit quality in peach and apricot. Adv. Hortic. Sci. [rivista dell'Ortofloroftutticoltura Ital., 4 (N.2), 1000-1006. https://doi.org/10.1400/13970 
Biale, J.B. (1960). "Respiration of fruits", in Handbook der plantephysiologie, ed. J.Wolf (Berlin (Germany): Springer- Verleg), 536-592.

Bizjak, J., Mikulic-Petkovsek, M., Stampar, F., and Veberic, R. (2013). Changes in primary metabolites and polyphenols in the peel of "braeburn" Apples (Malus domestica Borkh.) during advanced maturation. J. Agric. Food Chem., 61, 10283-10292. https://doi.org/10.1021/jf403064p

Bolin, H.R., and Stafford, A.E. (1974). Effect of processing and storage on provitamin A and vitamin C in apricots. J. Food Sci., 39, 1034-1036. https://doi.org/10.1111/j.1365-2621.1974.tb07305.x

Bomblies, K., and Madlung, A. (2014). Polyploidy in the Arabidopsis genus. Chromosom. Res., 22, 117134. https://doi.org/10.1007/s10577-014-9416-x

Bomblies, K., and Weigel, D. (2007). Arabidopsis-a model genus for speciation. Curr. Opin. Genet. Dev., 17, 500-504. https://doi.org/10.1016/j.gde.2007.09.006

Borsani, J., Budde, C.O., Porrini, L., Lauxmann, M.A., Lombardo, V.A., Murray, R., et al. (2009). Carbon metabolism of peach fruit after harvest: Changes in enzymes involved in organic acid and sugar level modifications. J. Exp. Bot., 60, 1823-1837. https://doi.org/10.1093/jxb/erp055

Bourguiba, H., Audergon, J. M., Krichen, L., Trifi-Farah, N., Mamouni, A., Trabelsi, S., et al. (2012). Loss of genetic diversity as a signature of apricot domestication and diffusion into the Mediterranean Basin. BMC Plant Biol., 12:49. https://doi.org/10.1186/1471-2229-12-49

Bourguiba, H., Scotti, I., Sauvage, C., Zhebentyayeva, T., Ledbetter, C., Krška, B., et al. (2020). Genetic structure of a worldwide germplasm collection of Prunus armeniaca L. reveals three major diffusion routes for varieties coming from the species' center of origin. Front Plant Sci., 11:638. https://doi.org/10.3389/fpls.2020.00638

Boyer, J., Liu, R.H. (2004). Apple phytochemicals and their health benefits. Nutr. J., 3, 5-19. https://doi.org/10.1186/1475-2891-3-5

Brady, C.J., (1987). Fruit ripening. Annu. Rev. Plant Physiol., 38, 155-178. https://doi.org/10.1146/annurev.pp.38.060187.001103

Brooks, R.M., and Olmo, H.P. (1997). The Brooks and Olmo register of fruit and nut varieties. 3rd ed.. (Alexandria (VA ):ASHS Press). ISBN: 9780961502744.

Bureau, S., Renard, C.M.G.C., Reich, M., Ginies, C., and Audergon, J.M. (2009). Change in anthocyanin concentrations in red apricot fruits during ripening. LWT - Food Sci. Technol., 42, 372-377. https://doi.org/10.1016/j.Iwt.2008.03.010 
Byrne, D.H. (2012). "Trends in fruit breeding", in Fruit Breeding, eds. M.L. Badenes and A. Hutagalung (Boston, MA (US): Springer). https://doi.org/10.1007/978-1-4419-0763-9

Caliskan, O., Bayazit, S., Sumbul, A. (2012). Fruit quality and phytochemical attributes of some apricot (Prunus armeniaca L.) cultivars as affected by genotypes and seasons. Not. Bot. Horti Agrobot. Cluj-Napoca., 40, 284-294. https://doi.org/10.15835/nbha4028044

Campbell, O.E., and Padilla-Zakour, O.I. (2013). Phenolic and carotenoid composition of canned peaches (Prunus persica) and apricots (Prunus armeniaca) as affected by variety and peeling. Food Res. Int., 54, 448-455. https://doi.org/10.1016/j.foodres.2013.07.016

Campoy, J.A., Ruiz, D., Egea, J., Rees, D.J.G., Celton, J.M., and Martínez-Gómez, P. (2011). inheritance of flowering time in apricot (Prunus armeniaca L.) and analysis of linked Quantitative Trait Loci (QTLs) using Simple Sequence Repeat (SSR) markers. Plant Mol. Biol. Report., 29, 404-410. https://doi.org/10.1007/s11105-010-0242-9

Camps, C., and Christen, D. (2009). Non-destructive assessment of apricot fruit quality by portable visible-near infrared spectroscopy. LWT - Food Sci. Technol., 42, 1125-1131. https://doi.org/10.1016/j.Iwt.2009.01.015

Cano, A., and Bermejo, A. (2011). Influence of rootstock and cultivar on bioactive compounds in citrus peels. J. Sci. Food Agric., 91, 1702-1711. https://doi.org/10.1002/jsfa.4375

Cano, A., Medina, A., and Bermejo, A. (2008). Bioactive compounds in different citrus varieties. Discrimination among cultivars. J. Food Compos. Anal., 21, 377-381. https://doi.org/10.1016/j.jfca.2008.03.005

Chen, F.X., Liu, X.H., Chen, L.S. (2009). Developmental changes in pulp organic acid concentration and activities of acid-metabolising enzymes during the fruit development of two loquat (Eriobotrya japonica Lindl.) cultivars differing in fruit acidity. Food Chem., 114, 657-664. https://doi.org/10.1016/J.FOODCHEM.2008.10.003

Cirilli, M., Bassi, D., and Ciacciulli, A. (2016). Sugars in peach fruit: A breeding perspective. Hortic. Res., https://doi.org/10.1038/hortres.2015.67

Colaric, M., Veberic, R., Stampar, F., and Hudina, M. (2005). Evaluation of peach and nectarine fruit quality and correlations between sensory and chemical attributes. J. Sci. Food Agric., 85, 26112616. https://doi.org/10.1002/JSFA.2316 
CPVO and EU. (2008). "European Union. CPVO-TP/070/2", in Merger Control Worldwide, eds. M.M. Dabbah, K.P.E. Lasok Q.C. (Cambridge: Cambridge University Press), 41-44. https://doi.org/10.1017/СBO9780511494697.014

Crisosto, C.H., Crisosto, G.M., and Metheney, P. (2003). Consumer acceptance of 'Brooks' and 'Bing' cherries is mainly dependent on fruit SSC and visual skin color. Postharvest Biol. Technol., 28, 159-167. https://doi.org/10.1016/S0925-5214(02)00173-4

D’Ambrosio, C., Arena, S., Rocco, M., Verrillo, F., Novi, G., Viscosi, V., et al. (2013). Proteomic analysis of apricot fruit during ripening. J. Proteomics, 78, 39-57. https://doi.org/10.1016/J.JPROT.2012.11.008

Del Pozo, J.C., and Ramírez-Parra, E. (2015). Whole genome duplications in plants: An overview from Arabidopsis. J. Exp. Bot., 66, 6991-7003. https://doi.org/10.1093/jxb/erv432

Deng, G.-F., Shen, C., Xu, X.-R., Kuang, R.-D., Guo, Y.-J., Zeng, L.-S., et al. (2012). Potential of fruit wastes as natural resources of bioactive compounds. Int. J. Mol. Sci., 13, 8308-8323. https://doi.org/10.3390/ijms13078308

Desnoues, E., Baldazzi, V., Génard, M., Mauroux, J.-B., Lambert, P., Confolent, C., et al. (2016). Dynamic QTLs for sugars and enzyme activities provide an overview of genetic control of sugar metabolism during peach fruit development. J. Exp. Bot., 67, 3419-3431. https://doi.org/10.1093/jxb/erw169

Desnoues, E., Gibon, Y., Baldazzi, V., Signoret, V., Génard, M., and Quilot-Turion, B. (2014). Profiling sugar metabolism during fruit development in a peach progeny with different fructose-to-glucose ratios. BMC Plant Biol., 14, 1-13. https://doi.org/10.1186/s12870-014-0336-x

Dillard, C.J., and German, J.B. (2000). Phytochemicals: Nutraceuticals and human health. J. Sci. Food Agric., 80, 1744-1756. https://doi.org/10.1002/1097-0010(20000915)80:12<1744::AIDJSFA725>3.0.CO;2-W

Dirlewanger, E., Cosson, P., Renaud, C., Monet, R., Poëssel, J.L., and Moing, A. (2006). New detection Of QTLs controlling major fruit quality components in peach. Acta Hortic., 713, 65-72. https://doi.org/10.17660/ActaHortic.2006.713.5

Dirlewanger, E., Graziano, E., Joobeur, T., Garriga-Calderé, F., Cosson, P., Howad, W., et al. (2004). Comparative mapping and marker-assisted selection in Rosaceae fruit crops. Proc. Natl. Acad. Sci., U. S. A. 101, 9891-9896. https://doi.org/10.1073/PNAS.0307937101 
Dirlewanger, E., Moing, A., Rothan, C., Svanella, L., Pronier, V., Guye, A., et al. (1999). Mapping QTLs controlling fruit quality in peach (Prunus persica (L.) Batsch). Theor. Appl. Genet. 98, 18-31. https://doi.org/10.1007/s001220051035

Dolenc-Šturm, K., Štampar, F., and Usenik, V. (1999). Evaluating of some quality parameters of different apricot cultibars using HPLC method. Accredit. Qual. Assur., 4, 297-309.

Dossett, M., Lee, J., and Finn, C.E. (2011). Characterization of a novel anthocyanin profile in wild black raspberry mutants: an opportunity for studying the genetic control of pigment and color. J. Funct. Foods, 3, 207-214. https://doi.org/10.1016/j.jff.2011.04.003

Dragovic-Uzelac, V., Levaj, B., Mrkic, V., Bursac, D., and Boras, M. (2007). The content of polyphenols and carotenoids in three apricot cultivars depending on stage of maturity and geographical region. Food Chem., 102, 966-975. https://doi.org/10.1016/j.foodchem.2006.04.001

Drogoudi, P.D., Vemmos, S., Pantelidis, G., Petri, E., Tzoutzoukou, C., and Karayiannis, I. (2008). Physical characters and antioxidant, sugar, and mineral nutrient contents in fruit from apricot (Prunus armeniaca L.) cultivars and hybrids. J. Agric. Food Chem., 56, 10754-10760. https://doi.org/10.1021/jf801995x

Edger, P.P., and Pires, J.C. (2009). Gene and genome duplications: the impact of dosage-sensitivity on the fate of nuclear genes. Chromosom. Res., 17, 699-717. https://doi.org/10.1007/s10577-0099055-9

Egea, J., Dicenta, F., and Burgos, L. (2004a). 'Rojo Pasión' Apricot. HortScience, 39, 1490-1491. https://doi.org/10.21273/HORTSCI.39.6.1490

Egea, J., Dicenta, F., Burgos, L., Martínez-Gómez, P., Rubio, M., Campoy, J.A., et al. (2010). New apricot cultivars from CEBAS-CSIC (Murcia, Spain) breeding programme. Acta Hortic., 862, 113-118. https://doi.org/10.17660/actahortic.2010.862.17

Egea, J., Martínez-Gómez, P., Dicenta, F., and Burgos, L. (2004b). 'Selene' Apricot. HortScience, 39, 1492-1493. https://doi.org/10.21273/HORTSCI.39.6.1492

Egea, J., Ruiz, D., and Burgos, L. (2005). "Dorada" Apricot. HortScience, 40, 1919-1920. https://doi.org/10.21273/HORTSCI.40.6.1919

Erdogan-Orhan, I., and Kartal, M. (2011). Insights into research on phytochemistry and biological activities of Prunus armeniaca L. (apricot). Food Res. Int., 44, 1238-1243. https://doi.org/10.1016/j.foodres.2010.11.014 
Eriksson, L., Johansson, E., Kettapeh-Wold, S., Wold, S. (1999). Introduction to multi- and megavariate data analysis using projection methods (PCA \& PLS). (Ümea: Umetrics).

Etienne, C., Rothan, C., Moing, A., Plomion, C., Bodénès, C., Svanella-Dumas, L., et al. (2002). Candidate genes and QTLs for sugar and organic acid content in peach [Prunus persica (L.) Batsch]. Theor. Appl. Genet., 105, 145-159. https://doi.org/10.1007/s00122-001-0841-9

Fan, X., Jiao, W., Wang, X., Cao, J., and Jiang, W. (2018). Polyphenol composition and antioxidant capacity in pulp and peel of apricot fruits of various varieties and maturity stages at harvest. Int. J. Food Sci. Technol., 53, 327-336. https://doi.org/10.1111/ijfs.13589

Fan, X., Zhao, H., Wang, X., Cao, J., and Jiang, W. (2017). Sugar and organic acid composition of apricot and their contribution to sensory quality and consumer satisfaction. Sci. Hortic., 225, 553-560. https://doi.org/10.1016/j.scienta.2017.07.016

FAOSTAT, 2021. Food and Agriculture Organization of the United Nations [WWW Document]. URL http://www.fao.org/faostat/en/\#home (accessed 7.18.21).

Fenech, M., Amaya, I., Valpuesta, V., and Botella, M.A. (2019). Vitamin C content in fruits: biosynthesis and regulation. front. Plant Sci. https://doi.org/10.3389/FPLS.2018.02006

Ferrer, J.L., Austin, M.B., Stewart, C., and Noel, J.P. (2008). Structure and function of enzymes involved in the biosynthesis of phenylpropanoids. Plant Physiol. Biochem., 46, 356-370. https://doi.org/10.1016/j.plaphy.2007.12.009

Fraser, C.M., and Chapple, C. (2011). The phenylpropanoid pathway in Arabidopsis. Arab. B., 9, e0152. https://doi.org/10.1199/tab.0152

Fu, L., Xu, B.T., Xu, X.R., Qin, X.S., Gan, R.Y., Li, H., et al. (2010). Antioxidant capacities and total phenolic contents of 56 wild fruits from South China. Molecules, 15, 8602-8617. https://doi.org/10.3390/molecules15128602

Fujisawa, M., Nakano, T., Shima, Y., and Ito, Y. (2013). A Large-Scale identification of direct targets of the tomato MADS Box transcription factor ripening inhibitor reveals the regulation of fruit ripening. Plant Cell, 25, 371-386. https://doi.org/10.1105/TPC.112.108118

Fukasawa-Akada, T., Kung Shain-dow, and Watson, J.C. (1996). Phenylalanine ammonia-lyase gene structure, expression, and evolution in Nicotiana. Plant Mol. Biol., 30, 711-722. https://doi.org/10.1007/BF00019006 
Gan, R.Y., Deng, Z.Q., Yan, A.X., Shah, N.P., Lui, W.Y., Chan, C.L., et al. (2016). Pigmented edible bean coats as natural sources of polyphenols with antioxidant and antibacterial effects. Lwt, 73, 168177. https://doi.org/10.1016/j.Iwt.2016.06.012

García, J.A., and Cambra, M. (2007). Plum pox virus and sharka disease. Plant Viruses, 1, 69-79.

García, J.A., Glasa, M., Cambra, M., and Candresse, T. (2014). Plum pox virus and sharka: a model potyvirus and a major disease. Mol. Plant Pathol., 15, 226-241. https://doi.org/10.1111/mpp.12083

García-Gómez, B.E., Razi, M., Salazar, J.A., Prudencio, A.S., Ruiz, D., Dondini, L., et al. (2018). Comparative analysis of SSR markers developed in exon, intron, and intergenic regions and distributed in regions controlling fruit quality traits in Prunus species: genetic diversity and association studies. Plant Mol. Biol. Report., 36, 23-35. https://doi.org/10.1007/s11105-0171058-7

García-Gómez, B.E., Salazar, J.A., Dondini, L., Martínez-Gómez, P., and Ruiz, D. (2019). Identification of QTLs linked to fruit quality traits in apricot (Prunus armeniaca $\mathrm{L}$.) and biological validation through gene expression analysis using qPCR. Mol. Breed., 39, 28. https://doi.org/10.1007/s11032-0180926-7

García-Gómez, B.E., Salazar, J.A., Nicolás-Almansa, M., Razi, M., Rubio, M., Ruiz, D., et al. (2021). Molecular bases of fruit quality in Prunus species: an integrated genomic, transcriptomic, and metabolic review with a breeding perspective. Int. J. Mol. Sci., 22(1), 333. https://doi.org/10.3390/ijms22010333

Giada, M.L.R. (2013). "Food phenolic compounds: main classes, sources and their antioxidant power", in Oxidative stress chronic degenerative diseases: a role for antioxidants, ed. J.A. MorálezGonzález (Intech Publisher). https://doi.org/10.5772/51687

Gil, M.I., Tomás-Barberán, F.A., Hess-Pierce, B., and Kader, A.A. (2002). Antioxidant capacities, phenolic compounds, carotenoids, and vitamin C contents of nectarine, peach, and plum cultivars from California. J. Agric. Food Chem., 50, 4976-4982. https://doi.org/10.1021/jf020136b

Ginter, E., and Simko, V. (2012). Plant polyphenols in prevention of heart disease. Bratis/ Lek Listy,. 113 (8), 476-480. https://doi.org/10.4149/bll_2012_105

Gómez, G.E., and Ligarreto, G.A. (2012). Analysis of genetic effects of major genes on yield traits of a pea (Pisum sativum L.) cross between the Santa Isabel x WSU 31 varieties. Agron. Colomb., 30, 317-325. 
Gómez-Martínez, H., Bermejo, A., Zuriaga, E., and Badenes, M.L. (2021). Polyphenol content in apricot fruits. Sci. Hortic., 277, 109828. https://doi.org/10.1016/j.scienta.2020.109828

Graetz, D. (2018). Selecting and releasing to industry high quality fresh and dried apricots for export and domestic markets. Summary project report for SARDI Executive Director. (Urrbrae (South Australia): South Australian Research Development Institute. Sustainable Systems).

Graves, S., Piepho, H.P., Selzer, L., and Dorai-Raj, S. (2019). R package. multcompView: Visualizations of Paired Comparisons. https://cran.r-project.org/web/packages/multcompView/index.html

Groppi, A., Liu, S., Cornille, A., Decroocq, S., Bui, Q.T., Tricon, D., et al. (2021). Population genomics of apricots unravels domestication history and adaptive events. Nat. Commun., 12, 3956. https://doi.org/10.1038/s41467-021-24283-6

Gündoğdu, M., Kan, T., and Gecer, M.K. (2013). Vitamins, flavonoids, and phenolic acid levels in earlyand late-ripening apricot (Prunus armeniaca L.) cultivars from Turkey. HortScience, 48, 696-700. https://doi.org/10.21273/hortsci.48.6.696

Güneș, N.T. (2006). Frost hardiness of some Turkish apricot cultivars during the bloom period. HortScience, 41, 310-312. https://doi.org/10.21273/HORTSCI.41.2.310

Gurrieri, F., Audergon, J.M., Albagnac, G., and Reich, M. (2001). Soluble sugars and carboxylic acids in ripe apricot fruit as parameters for distinguishing different cultivars. Euphytica, 117, 183-189. https://doi.org/10.1023/A:1026595528044

Hamberger, B., Ellis, M., Friedmann, M., de Azevedo Souza, C., Barbazuk, B., and Douglas, C.J. (2007). Genome-wide analyses of phenylpropanoid-related genes in Populus trichocarpa, Arabidopsis thaliana and Oryza sativa: the Populus lignin toolbox and conservation and diversification of angiosperm gene families. Can. J. Bot., 85, 1182-1201. https://doi.org/10.1139/B07-098

Harborne, J.B. (2009). "Plant Secondary Metabolism", in Plant Ecology, Crawley, M.J. (Oxford (UK): Blackwell Publishing Ltd.), 132-155. https://doi.org/10.1002/9781444313642.ch5

Hartmann, U., Sagasser, M., Mehrtens, F., Stracke, R., and Weisshaar, B. (2005). Differential combinatorial interactions of cis-acting elements recognized by R2R3-MYB, BZIP, and BHLH factors control light-responsive and tissue-specific activation of phenylpropanoid biosynthesis genes. Plant Mol. Biol., 57, 155-171. https://doi.org/10.1007/s11103-004-6910-0 
Hegedüs, A., Engel, R., Abrankó, L., Balogh, E., Blázovics, A., Hermán, R., et al. (2010). Antioxidant and antiradical capacities in apricot (Prunus armeniaca L.) fruits: variations from genotypes, years, and analytical methods. J. Food Sci., 75, C722-C730. https://doi.org/10.1111/j.17503841.2010.01826.x

Hegedüs, A., Pfeiffer, P., Papp, N., Abrankó, L., Blázovics, A., Pedryc, A., et al. (2011). Accumulation of antioxidants in apricot fruit through ripening: characterization of a genotype with enhanced functional properties. Biol. Res., 44, 339-344. https://doi.org/10.4067/S071697602011000400004

Hernández, F., Pinochet, J., Moreno, M.A., Martínez, J.J., and Legua, P. (2010). Performance of Prunus rootstocks for apricot in Mediterranean conditions. Sci. Hortic., 124, 354-359. https://doi.org/10.1016/j.scienta.2010.01.020

Hernández Mora, J.R., Micheletti, D., Bink, M., van de Weg, E., Cantín, C., Nazzicari, N., et al. (2017). Integrated QTL detection for key breeding traits in multiple peach progenies. BMC Genomics, 18 , 1-15. https://doi.org/10.1186/s12864-017-3783-6

Higo, K., Ugawa, Y., Iwamoto, M., and Korenaga, T. (1999). Plant cis-acting regulatory DNA elements (PLACE) database: 1999. Nucleic Acids Res., 27, 297-300. https://doi.org/10.1093/nar/27.1.297

Hu, F., Stampfer, F., Manson, J., Grodstein, F., Colditz, G., Speizer, F., et al. (2000). Trends in the incidence of coronary heart disease and changes in diet and lifestyle in women. N. Engl. J. Med., 343, 530-537. https://doi.org/10.1056/NEJM200008243430802

Huang, Y., Gou, J., Jia, Z., Yang, L., Sun, Y., Xiao, X., et al. (2012). Molecular cloning and characterization of two genes encoding dihydroflavonol-4-reductase from Populus trichocarpa. PLoS One, 7, 1-8. https://doi.org/10.1371/journal.pone.0030364

Huang, Z., Wang, Q., Xia, L., Hui, J., Li, J., Feng, Y., et al. (2019). Preliminarily exploring of the association between sugars and anthocyanin accumulation in apricot fruit during ripening. Sci. Hortic., 248, 112-117. https://doi.org/10.1016/j.scienta.2019.01.012

Illa, E., Eduardo, I., Audergon, J.M., Barale, F., Dirlewanger, E., Li, X., et al. (2011). Saturating the Prunus (stone fruits) genome with candidate genes for fruit quality. Mol. Breed., 28, 667-682. https://doi.org/10.1007/s11032-010-9518-x

Irisarri, P., Zhebentyayeva, T., Errea, P., and Pina, A. (2016). Differential expression of phenylalanine ammonia lyase (PAL) genes implies distinct roles in development of graft incompatibility symptoms in Prunus. Sci. Hortic., 204, 16-24. https://doi.org/10.1016/j.scienta.2016.03.025 
Jaillon, O., Aury, J.M., Noel, B., Policriti, A., Clepet, C., Casagrande, A. et al. (2007). The grapevine genome sequence suggests ancestral hexaploidization in major angiosperm phyla. Nature, 449, 463-467. https://doi.org/10.1038/nature06148

Jiang, F., Zhang, J., Wang, S., Yang, L., Luo, Y., Gao, S., et al. (2019). The apricot (Prunus armeniaca L.) genome elucidates Rosaceae evolution and beta-carotenoid synthesis. Hortic. Res., 6, 128. https://doi.org/10.1038/s41438-019-0215-6

Jiao, Y., Ma, R.J., Shen, Z.J-, Yan, J., and Yu, M.L. (2014). Gene regulation of anthocyanin biosynthesis in two blood-flesh peach (Prunus persica (L.) Batsch) cultivars during fruit development. J. Zhejiang Univ. Sci. B, 15, 809-819. https://doi.org/10.1631/jzus.B1400086

Jin, X., Huang, H., Wang, L., Sun, Y., and Dai, S. (2016). Transcriptomics and metabolite analysis reveals the molecular mechanism of anthocyanin biosynthesis branch pathway in different senecio cruentus cultivars. Front. Plant Sci., 7, 1-14. https://doi.org/10.3389/fpls.2016.01307

Jin, U.H., Lee, J.Y., Kang, S.K., Kim, J.K., Park, W.H., Kim, J.G., et al. (2005). A phenolic compound, 5caffeoylquinic acid (chlorogenic acid), is a new type and strong matrix metalloproteinase- 9 inhibitor: Isolation and identification from methanol extract of Euonymus alatus. Life Sci., 77, 2760-2769. https://doi.org/10.1016/j.lfs.2005.02.028

Joobeur, T., Viruel, M.A., de Vicente, M.C., Jáuregui, B., Ballester, J., Dettori, M.T., et al. (1998). Construction of a saturated linkage map for Prunus using an almond x peach F2 progeny. Theor. Appl. Genet., 97, 1034-1041. https://doi.org/10.1007/s001220050988

Jung, S., Lee, T., Cheng, C.H., Buble, K., Zheng, P., Yu, J., et al. (2019). 15 years of GDR: New data and functionality in the Genome Database for Rosaceae. Nucleic Acids Res., 47, D1137-D1145. https://doi.org/10.1093/nar/gky1000

Kader, A.A. (2008). Flavor quality of fruits and vegetables. J. Sci. Food Agric., 88, 1863-1868. https://doi.org/10.1002/JSFA.3293

Kanayama, Y., Kogawa, M., Yamaguchi, M., and Kanahama, K. (2005). Fructose content and the activity of fructose-related enzymes in the fruit of eating-quality peach cultivars and native-type peach cultivars. J. Japanese Soc. Hortic. Sci., 74, 431-436. https://doi.org/10.2503/jjshs.74.431

Karabulut, I., Bilenler, T., Sislioglu, K., Gokbulut, I., Seyhan, F., Ozdemir, I.S., et al. (2018). Effect of fruit canopy positions on the properties of apricot (Prunus armeniaca L.) varieties. J. Food Biochem., 42, 1-12. https://doi.org/10.1111/jfbc.12458 
Karayiannis, I. (2006a). Progress in apricot breeding for resistance to Sharka disease (plum pox virus, PPV) in Greece. Acta Hortic., 93-96. https://doi.org/10.17660/ActaHortic.2006.717.17

Karayiannis, I., Mainou, A., Stylianidis, D., Thomidis, T., Karayiannis, N.I., and Tsaftaris, A. (2006b). Resistant to Sharka disease (PPV) apricot hybrids of high quality, selected in Greece. Acta Hortic., 701 I, 337-340. https://doi.org/10.17660/ACTAHORTIC.2006.701.53

Karlova, R., Chapman, N., David, K., Angenent, G.C., Seymour, G.B., and de Maagd, R.A. (2014). Transcriptional control of fleshy fruit development and ripening. J. Exp. Bot. 65, 4527-4541. https://doi.org/10.1093/jxb/eru316

Kassambara, A., Mundt, F. 2020. R package. factoextra: Extract and Visualize the Results of Multivariate Data Analyses. https://cran.r-project.org/web/packages/factoextra/index.html

Kayesh, E., Shangguan, L., Korir, N.K., Sun, X., Bilkish, N., Zhang, Y., et al. (2013). Fruit skin color and the role of anthocyanin. Acta Physiol. Plant., 35, 2879-2890. https://doi.org/10.1007/s11738013-1332-8

Kennedy, J.A., Matthews, M.A., and Waterhouse, A.L. (2000). Changes in grape seed polyphenols during fruit ripening. Phytochemistry, 55, 77-85. https://doi.org/10.1016/S0031-9422(00)001965

Kim, D.O., Jeong, S.W., and Lee, C.Y. (2003). Antioxidant capacity of phenolic phytochemicals from various cultivars of plums. Food Chem., 81, 321-326. https://doi.org/10.1016/S03088146(02)00423-5

Klee, H.J. (2010). Improving the flavor of fresh fruits: genomics, biochemistry, and biotechnology. New Phytol., 187, 44-56. https://doi.org/10.1111/J.1469-8137.2010.03281.X

Kostina K.F. (1964). Application the phytogeographical method to apricot classification. Trud. Nikit. Bot. Gard., 24.

Kroger, M., Meister, K., and Kava, R. (2006). Low-calorie sweeteners and other sugar substitutes: a review of the safety issues. Compr. Rev. Food Sci. Food Saf., 5, 35-47. https://doi.org/10.1111/J.1541-4337.2006.TB00081.X

Kuhn, B.M., Geisler, M., Bigler, L., and Ringli, C. (2011). Flavonols accumulate asymmetrically and affect auxin transport in Arabidopsis. Plant Physiol., 156, 585-595. https://doi.org/10.1104/pp.111.175976 
Kumar, S., Stecher, G., Li, M., Knyaz, C., and Tamura, K. (2018). MEGA X: Molecular evolutionary genetics analysis across computing platforms. Mol. Biol. Evol., 35, 1547-1549. https://doi.org/10.1093/molbev/msy096

Kumar, K., Yadav, A.N., Kumar, V., Vyas, P., and Dhaliwal, H.S. (2017). Food waste: a potential bioresource for extraction of nutraceuticals and bioactive compounds. Bioresour. Bioprocess. https://doi.org/10.1186/s40643-017-0148-6

Layne R.E.C., Bailey C.H., and Hough L.F.. (1996). "Apricots", in Fruit Breeding, Vol. 1. Tree and Tropical Fruits, eds. J. Janick and J.N. Moore (New York: John Wiley \& Sons, Inc),79-111.

Leccese, A., Bartolini, S., and Viti, R. (2008). Total antioxidant capacity and phenolics content in fresh apricots. Acta Aliment. 37, 65-76. https://doi.org/10.1556/AAlim.37.2008.1.6

Ledbetter, C.A. (2008). "Apricots", in Temperate Fruit Crop Breeding, ed. J.F. Hancock. (Dordrecht (Netherlands): Springer), 39-82. https://doi.org/10.1007/978-1-4020-6907-9_2

Ledbetter, C.A. (2010). Apricot breeding in North America: current status and future prospects. Acta Hortic. 862, 85-92. https://doi.org/10.17660/ActaHortic.2010.862.12

Lee, S.K., and Kader, A.A. (2000). Preharvest and postharvest factors influencing vitamin C content of horticultural crops. Postharvest Biol. Technol., 20, 207-220. https://doi.org/10.1016/S0925$5214(00) 00133-2$

Li, X., Wang, T., Zhou, B., Gao, W., Cao, J., and Huang, L. (2014). Chemical composition and antioxidant and anti-inflammatory potential of peels and flesh from 10 different pear varieties (Pyrus spp.). Food Chem., 152, 531-538. https://doi.org/10.1016/J.FOODCHEM.2013.12.010

Lian, S., Zhou, Y., Liu, Z., Gong, A., and Cheng, L. (2020). The differential expression patterns of paralogs in response to stresses indicate expression and sequence divergences. BMC Plant Biol. 20, 1-16. https://doi.org/10.1186/s12870-020-02460-x

Lima, G.P.P., Vianello, F., Corrêa, C.R., Campos, R.A.S., and Borguini, M.G. (2014). Polyphenols in fruits and vegetables and its effect on human health. Food Nutr. Sci., 05, 1065-1082. https://doi.org/10.4236/fns.2014.511117

Lloret, A., Conejero, A., Lleida, C., Petri, C., Gil-Muñoz, F., Burgos, L., et al. (2017). Dual regulation of water retention and cell growth by a stress-associated protein (SAP) gene in Prunus. Sci. Rep., 7, 1-15. https://doi.org/10.1038/s41598-017-00471-7 
Lo Piero, A.R., Puglisi, I., and Petrone, G. (2006). Gene characterization, analysis of expression and in vitro synthesis of dihydroflavonol 4-reductase from [Citrus sinensis (L.) Osbeck]. Phytochemistry, 67, 684-695. https://doi.org/10.1016/j.phytochem.2006.01.025

Luo, P., Ning, G., Wang, Z., Shen, Y., Jin, H., Li, P., et al. (2016). Disequilibrium of flavonol synthase and dihydroflavonol-4-reductase expression associated tightly to white vs. red color flower formation in plants. Front. Plant Sci. 6, 1-12. https://doi.org/10.3389/fpls.2015.01257

Magwaza, L.S., and Opara, U.L. (2015). Analytical methods for determination of sugars and sweetness of horticultural products: a review. Sci. Hortic. https://doi.org/10.1016/j.scienta.2015.01.001

Manach, C., Mazur, A., and Scalbert, A. (2005). Polyphenols and prevention of cardiovascular diseases. Curr. Opin. Lipidol. https://doi.org/10.1097/00041433-200502000-00013

Manach, C., Scalbert, A., Morand, C., Rémésy, C., and Jiménez, L. (2004). Polyphenols: food sources and bioavailability. Am. J. Clin. Nutr., 79 (5), 727-747. https://doi.org/10.1093/ajcn/79.5.727

Mangiafico, S. (2020). R package. rcompanion: Functions to Support Extension Education Program Evaluation. https://cran.r-project.org/web/packages/rcompanion/index.html

MAPA, 2020. Ministerio de Agricultura, Pesca y Alimentación [WWW Document]. URL https://www.mapa.gob.es/es/ministerio/default.aspx (accessed 7.18.21).

Martens, S., Knott, J., Seitz, C.A., Janvari, L., Yu, S.N., and Forkmann, G. (2003). Impact of biochemical pre-studies on specific metabolic engineering strategies of flavonoid biosynthesis in plant tissues. Biochem. Eng. J. 14, 227-235. https://doi.org/10.1016/S1369-703X(02)00224-3

Martínez-Calvo, J., Font, A., Llácer, G., and Badenes, M.L. (2009). Apricot and peach breeding programs from the IVIA. Acta Hortic., 814, 185-188. https://doi.org/10.17660/ActaHortic.2009.814.23

Martínez-Calvo, J., Llácer, G., and Badenes, M.L. (2010). "Rafel" and "Belgida", two apricot cultivars resistant to sharka. HortScience, 45, 1904-1905. https://doi.org/10.21273/hortsci.45.12.1904

Martínez-Gómez, P., Dicenta, F., and Audergon, J.M. (2000). Behaviour of apricot (Prunus armeniaca L.) cultivars in the presence of sharka (plum pox potyvirus): a review. Agronomie, 20, 407-422. https://doi.org/10.1051/AGRO:2000137

Martínez-Mora, C., Rodríguez, J., Cenis, J.L., and Ruiz-García, L. (2009). Variabilidad genética entre cultivares de albaricoquero tradicionales (Prunus armeniaca L.) del sureste español. Spanish J. Agric. Res., 7, 855-868. https://doi.org/10.5424/sjar/2009074-1099 
Matthes, A., and Schmitz-Eiberger, M. (2009). Polyphenol content and antioxidant capacity of apple fruit: Effect of cultivar and storage conditions. J. Appl. Bot. Food Qual., 82, 152-157.

Mesarović, J., Trifković, J., Tosti, T., Fotirić Akšić, M., Milatović, D., Ličina, V., et al. (2018). Relationship between ripening time and sugar content of apricot (Prunus armeniaca L.) kernels. Acta Physiol. Plant., 40, 0. https://doi.org/10.1007/s11738-018-2731-7

Moing, A., Renaud, C., Gaudillère, M., Raymond, P., Roudeillac, P., and Denoyes-Rothan, B. (2001). Biochemical changes during fruit development of four strawberry cultivars. J. Am. Soc. Hortic. Sci., 126, 394-403. https://doi.org/10.21273/JASHS.126.4.394

Mokrani, A., Krisa, S., Cluzet, S., da Costa, G., Temsamani, H., Renouf, E., et al. (2016). Phenolic contents and bioactive potential of peach fruit extracts. Food Chem., 202, 212-220. https://doi.org/10.1016/j.foodchem.2015.12.026

Moldes, A.B., Vecino, X., and Cruz, J.M. (2017). Nutraceuticals and food additives, in: current developments in biotechnology and bioengineering. Elsevier, 143-164. https://doi.org/10.1016/B978-0-444-63666-9.00006-6

Mole, S., Ross, J.A.M., and Waterman, P.G. (1988). Light-induced variation in phenolic levels in foliage of rain-forest plants. J. Chem. Ecol., 14, 1-21. https://doi.org/10.1007/BF01022527

Moustafa, K., and Cross, J. (2019). Production, pomological and nutraceutical properties of apricot. J. Food Sci. Technol., 56, 12-23. https://doi.org/10.1007/s13197-018-3481-7

Muñoz-Sanz, J.V., López, I., Badenes, M. L., Romero, C., and Zuriaga, E. (2020a). Selección asistida por marcadores para obtener albaricoques autocompatibles. Nota Técnica. IVIA-Centro de Citricultura y Protección Vegetal (Moncada (València, España): Generelalitat Valenciana).

Muñoz-Sanz J.V., Zuriaga E., Badenes M.L., and Romero C. 2017a. A disulfide bond A-like oxidoreductase is a strong candidate gene for self-incompatibility in apricot (Prunus armeniaca) pollen. Journal of Experimental Botany, 68 (18), 5069-5078, https://doi.org/10.1093/jxb/erx336

Muñoz-Sanz J.V., Zuriaga E., Cruz-García F., McClure B., and Romero C. 2020b. Self-(In)compatibility Systems: target traits for crop-production, plant breeding, and Biotechnology. Front Plant Sci., 11:195. https://doi.org/10.3389/fpls.2020.00195

Muñoz-Sanz, J.V., Zuriaga, E., López, I., Badenes, M.L., and Romero, C., 2017b. Self-(in)compatibility in apricot germplasm is controlled by two major loci, S and M. BMC Plant Biol. 17, 82. https://doi.org/10.1186/s12870-017-1027-1 
$\begin{array}{llllll}\text { Murrell, } & \text { P. (2005). } & \text { R } & \text { graphics. Chapman }\end{array}$ https://www.stat.auckland.ac.nz/ paul/RGraphics/rgraphics.html

Naryal, A., Angmo, S., Angmo, P., Kant, A., Chaurasia, O.P., and Stobdan, T. (2019). Sensory attributes and consumer appreciation of fresh apricots with white seed coats. Hortic. Environ. Biotechnol., 60, 603-610. https://doi.org/10.1007/s13580-019-00146-4

NCBI, 2021. National Center for Biotechnology Information [WWW Document]. URL https://www.ncbi.nlm.nih.gov/ (accessed 7.15.21).

Nicolle, C., Carnat, A., Fraisse, D., Lamaison, J.L., Rock, E., Michel, H., et al. (2004). Characterisation and variation of antioxidant micronutrients in lettuce (Lactuca sativa folium). J. Sci. Food Agric., 84, 2061-2069. https://doi.org/10.1002/jsfa.1916

Nunes, C., Guyot, S., Marnet, N., Barros, A.S., Saraiva, J.A., Renard, C.M.G.C., et al. (2008). Characterization of plum procyanidins by thiolytic depolymerization. J. Agric. Food Chem., 56, 5188-5196. https://doi.org/10.1021/JF8006135

Ogle, D.H., Doll, J.C., Wheeler, P., Dinno, A. (2020). FSA: Fisheries Stock Analysis. R package version 0.9.1. https://github.com/droglenc/FSA

Ogundiwin, E.A., Peace, C.P., Gradziel, T.M., Parfitt, D.E., Bliss, F.A., and Crisosto, C.H. (2009). A fruit quality gene map of Prunus. BMC Genomics, 10, 1-13. https://doi.org/10.1186/1471-2164-10587

Ogundiwin, E.A., Peace, C.P., Nicolet, C.M., Rashbrook, V.K., Gradziel, T.M., Bliss, F.A., et al. (2008). Leucoanthocyanidin dioxygenase gene $(P p L D O X)$ : a potential functional marker for cold storage browning in peach. Tree Genet. Genomes, 4, 543-554. https://doi.org/10.1007/s11295-0070130-0

Orero, G., Cuenca, J., Romero, C., Martinez-Calvo, J., Badenes, M.L., and Llacer, G. (2004). Selection of seedling rootstocks for apricot and almond. Acta Hortic., 658, 529-533 (658), 529-533. https://doi.org/10.17660/ActaHortic.2004.658.80

Osorio, S., Scossa, F., and Fernie, A. (2013). Molecular regulation of fruit ripening. Front. Plant Sci., 14. https://doi.org/10.3389/FPLS.2013.00198

Owais, S.J. (2010). Physical and chemical characteristics of apricot fruits grown in Southern Jordan. Jordan J. Agric. Sci., 3, 288-296. 
Owens, D.K., Alerding, A.B., Crosby, K.C., Bandara, A.B., Westwood, J.H., and Winkel, B.S.J. (2008). Functional analysis of a predicted flavonol synthase gene family in Arabidopsis. Plant Physiol., 147, 1046-1061. https://doi.org/10.1104/pp.108.117457

Pelletier, M.K., Burbulis, I.E., and Winkel-Shirley, B. (1999). Disruption of specific flavonoid genes enhances the accumulation of flavonoid enzymes and end-products in Arabidopsis seedlings. Plant Mol. Biol., 40, 45-54. https://doi.org/10.1023/A:1026414301100

Pennone, F., Abbate, V., Carbone, A., and Scarpato, L. (2010). Apricot Breeding in Caserta: results and perspectives. Acta Hortic., 67-73. https://doi.org/10.17660/actahortic.2010.862.8

Pérez-Pastor, A., Ruiz-Sánchez, Ma C., Domingo, R. and Torrecillas, A. (2004). Growth and phenological stages of Búlida apricot trees in south-east Spain. Agronomie, $24 \quad 2$ 93-100. https://doi.org/10.1051/agro:2004004

Petri, C., Scorza, R., and Srinivasan, C. (2012). "Highly efficient transformation protocol for plum (Prunus domestica L.)", in Transgenic Plants. Methods in Molecular Biology (Methods and Protocols), eds. J. Dunwell and A. Wetten (Humana Press). https://doi.org/10.1007/978-1-61779558-9_16

Petri, C., Wang, H., Alburquerque, N., Faize, M., and Burgos, L. (2008). Agrobacterium-mediated transformation of apricot (Prunus armeniaca L.) leaf explants. Plant Cell Rep., 27(8):1317-24. https://doi.org/10.1007/s00299-008-0550-9.

Piagnani, C.M., and Bassi, D. (2013). Fruit quality evaluation of diverse apricot cultivars. Aspects of Applied Biology, 119, 139-144.

Polak, J., Kominek, P., Krska, B., and Pivalova, J. (2008). Durable resistance of apricot cultivars Harlayne and Betinka to six different strains of Plum pox virus. J. Plant Pathol., 90, 37-40. https://doi.org/http://dx.doi.org/10.4454/jpp.v90i1sup.614

Polo-Oltra, Á., Romero, C., López, I., Badenes, M.L., and Zuriaga, E. (2020). Cost-effective and timeefficient molecular assisted selection for PPV resistance in apricot based on ParPMC2 allelespecific PCR. Agron., 10 (9), 1292. https://doi.org/10.3390/AGRONOMY10091292

Preece, J.E., and Aradhya, M. (2013). The Prunus collection at the National Clonal Germplasm Repository in Davis, California. Acta Hortic., 985, 47-54. https://doi.org/10.17660/ActaHortic.2013.985.5 
Quilot, B., Wu, B.H., Kervella, J., Génard, M., Foulongne, M., and Moreau, K. (2004). QTL analysis of quality traits in an advanced backcross between Prunus persica cultivars and the wild relative species P. davidiana. Theor. Appl. Genet., 109, 884-897. https://doi.org/10.1007/s00122-004$1703-z$

Radi, M., Mahrouz, M., Jaouad, A., Tacchini, M., Aubert, S., Hugues, M., et al. (1997). Phenolic composition, browning susceptibility, and carotenoid content of several apricot cultivars at maturity. HortScience., https://doi.org/10.21273/hortsci.32.6.1087

Raes, J., Rohde, A., Christensen, J.H., van de Peer, Y., and Boerjan, W. (2003). Genome-Wide characterization of the lignification toolbox in Arabidopsis. Plant Physiol., 133 (3), 1051-1071. https://doi.org/10.1104/pp.103.026484

Rahim, M.A., Busatto, N., and Trainotti, L. (2014). Regulation of anthocyanin biosynthesis in peach fruits. Planta, 240, 913-929. https://doi.org/10.1007/s00425-014-2078-2

Reichert, A.I., He, X.Z., and Dixon, R.A. (2009). Phenylalanine ammonia-lyase (PAL) from tobacco (Nicotiana tabacum): characterization of the four tobacco PAL genes and active heterotetrameric enzymes. Biochem. J., 424, 233-242. https://doi.org/10.1042/BJ20090620

Reig, G., Zarrouk, O., Font, C., and Moreno, M.Á. (2018). Anatomical graft compatibility study between apricot cultivars and different plum based rootstocks. Sci. Hortic., 237, 67-73. https://doi.org/10.1016/j.scienta.2018.03.035

Rice-Evans, C.A., Miller, N.J., and Paganga, G. (1997). Antioxidant properties of phenolic compounds. Trends Plant Sci. 2, 152-159. https://doi.org/10.1016/S1360-1385(97)01018-2

Rodríguez-Mateos, A., Vauzour, D., Krueger, C.G., Shanmuganayagam, D., Reed, J., Calani, L., et al. (2014). Bioavailability, bioactivity, and impact on health of dietary flavonoids and related compounds: an update. Arch. Toxicol., 88, 1803-1853. https://doi.org/10.1007/s00204-014$1330-7$

Romero, C., Pedryc, A., Muñoz, V., Llácer, G., and Badenes, M.L. (2003). Genetic diversity of different apricot geographical groups determined by SSR markers. Genome, 46, 244-252. https://doi.org/10.1139/g02-128

Romeu, J.F., Monforte, A.J., Sánchez, G., Granell, A., García-Brunton, J., Badenes, M.L., et al. (2014). Quantitative trait loci affecting reproductive phenology in peach. BMC Plant Biol., 14. https://doi.org/10.1186/1471-2229-14-52 
Roulin, A., Auer, P.L., Libault, M., Schlueter, J., Farmer, A., May, G., et al. (2013). The fate of duplicated genes in a polyploid plant genome. Plant J., 73, 143-153. https://doi.org/10.1111/tpj.12026

Roussos, P.A., Sefferou, V., Denaxa, N.K., Tsantili, E., and Stathis, V. (2011). Apricot (Prunus armeniaca L.) fruit quality attributes and phytochemicals under different crop load. Sci. Hortic., 129, 472478. https://doi.org/10.1016/j.scienta.2011.04.021

Rubio, M., Dicenta, F., García, A., Ruiz, D., Martínez-Gómez, P., and Egea, J. (2009). Obtención De Nuevas Variedades Resistentes. Origen y situación actual de la sharka en España. Ministerio de Medioambiente y Medio Rural y Marino, 1-13.

Ruiz, D., Campoy, J.A. and Egea, J. (2006). Chilling requirements of apricot varieties. Acta Hortic., 717, 67-70. https://doi.org/10.17660/ActaHortic.2006.717.10

Ruiz, D., and Egea, J. (2008). Phenotypic diversity and relationships of fruit quality traits in apricot (Prunus armeniaca L.) germplasm. Euphytica, 163, 143-158. https://doi.org/10.1007/s10681007-9640-y

Ruiz, D., Egea, J., Gil, M.I., and Tomás-Barberán, F.A. (2005). Characterization and quantitation of phenolic compounds in new apricot (Prunus armeniaca L.) varieties. J. Agric. Food Chem., 53, 9544-9552. https://doi.org/10.1021/jf051539p

Ruiz, D., Lambert, P., Audergon, J.M., Dondini, L., Tartarini, S., Adami, M., et al. (2010). Identification of QTLs for fruit quality traits in apricot. Acta Hortic., 587-592. https://doi.org/10.17660/ACTAHORTIC.2010.862.93

Sabu, M.C., and Kuttan, R., (2002). Anti-diabetic activity of medicinal plants and its relationship with their antioxidant property. J. Ethnopharmacol, 81, 155-160. https://doi.org/10.1016/S0378$8741(02) 00034-X$

Sajid, M., Khan, M.A., Bilal, W., Rab, A., Iqbal, Z., and Irshad khan, S. (2017). Einfluss der Blattbehandlung mit Ascorbinsäure auf die antioxidative Wirkung, auf chemische Eigenschaften und den Fruchtertrag von Pfirsichkultursorten. Gesunde Pflanz. 69, 113-121. https://doi.org/10.1007/s10343-017-0395-7

Salazar, J.A., Martinez-Gómez, P., Tartarini, S., Dondini, L., and Ruiz, D. (2017). Construction of linkage maps and analysis of QTL linked to phenology and fruit quality traits in two apricot progenies combining SSRs and SNPs. Acta Hortic., 1172, 385-389. https://doi.org/10.17660/ActaHortic.2017.1172.73 
Salazar, J.A., Ruiz, D., Campoy, J.A., Tartarini, S., Dondini, L., and Martínez-Gómez, P. (2016). Inheritance of reproductive phenology traits and related QTL identification in apricot. Tree Genetics \& Genomes, 12. https://doi.org/10.1007/S11295-016-1027-6

Salazar, J.A., Ruiz, D., Egea, J., and Martínez-Gómez, P. (2013). Transmission of fruit quality traits in apricot (Prunus armeniaca L.) and analysis of linked Quantitative Trait Loci (QTLs) using Simple Sequence Repeat (SSR) markers. Plant Mol. Biol. Report., 31, 1506-1517. https://doi.org/10.1007/s11105-013-0625-9

Scalbert, A., Manach, C., Morand, C., Rémésy, C., and Jiménez, L. (2005). Dietary polyphenols and the prevention of diseases. Crit. Rev. Food Sci. Nutr., 45 (4), 287-306. https://doi.org/10.1080/1040869059096

Schloerke, B., Cook, D., Larmarange, J., Briatte, F., Marbach, M., Thoen, E., et al. (2020). R package. GGally: Extension to 'ggplot2'. https://cloud.r-project.org/web/packages/GGally/index.html

Schmitzer, V., Slatnar, A., Mikulic-Petkovsek, M., Veberic, R., Krska, B., and Stampar, F. (2011). Comparative study of primary and secondary metabolites in apricot (Prunus armeniaca L.) cultivars. J. Sci. Food Agric., 91, 860-866. https://doi.org/10.1002/jsfa.4257

Sdiri, S., Bermejo, A., Aleza, P., Navarro, P., and Salvador, A. (2012). Phenolic composition, organic acids, sugars, vitamin $\mathrm{C}$ and antioxidant activity in the juice of two new triploid late-season $\begin{array}{lllll}\text { mandarins. } & \text { Food } & \text { Research }\end{array}$ https://doi.org/10.1016/J.FOODRES.2012.07.040

Seymour, G., Østergaard, L., Chapman, N., Knapp, S., and Martin, C. (2013). Fruit development and ripening. Annu. Rev. Plant Biol., 64, 219-241. https://doi.org/10.1146/ANNUREV-ARPLANT050312-120057

Shimada, N., Sasaki, R., Sato, S., Kaneko, T., Tabata, S., Aoki, T., et al. (2005). A comprehensive analysis of six dihydroflavonol 4-reductases encoded by a gene cluster of the Lotus japonicus genome. J. Exp. Bot., 56, 2573-2585. https://doi.org/10.1093/jxb/eri251

Shulaev, V., Sargent, D.J., Crowhurst, R.N., Mockler, T.C., Folkerts, O., Delcher, A.L., et al. (2011). The genome of woodland strawberry (Fragaria vesca). Nat. Genet., 43, 109-116. https://doi.org/10.1038/ng.740

Signorell, A., Aho, K., Alfons, A., Anderegg, N., Aragón, T., Arachchige, C., et al. (2020). DescTools: Tools for Descriptive Statistics. $\mathrm{R}$ package version 0.99.43. https://cran.rproject.org/package=DescTools. 
Singh, K., Kumar, S., Yadav, S.K., and Ahuja, P.S. (2009). Characterization of dihydroflavonol 4reductase cDNA in tea [Camellia sinensis (L.) O. Kuntze]. Plant Biotechnol. Rep., 3, 95-101. https://doi.org/10.1007/s11816-008-0079-y

Slavin, J., and Lloyd, B. (2012). Health benefits of fruits and vegetables. Adv. Nutr., 3, 506-516. https://doi.org/10.3945/AN.112.002154

Sochor, J., Zitka, O., Skutkova, H., Pavlik, D., Babula, P., Krska, B., et al. (2010). Content of phenolic compounds and antioxidant capacity in fruits of apricot genotypes. Molecules, 15, 6285. https://doi.org/10.3390/MOLECULES15096285

Socquet-Juglard, D., Christen, D., Devènes, G., Gessler, C., Duffy, B., and Patocchi, A. (2013). Mapping architectural, phenological, and fruit quality QTLs in apricot. Plant Mol. Biol. Report., 31, 387397. https://doi.org/10.1007/s11105-012-0511-x

Soriano, J.M., Domingo, M.L., Zuriaga, E., Romero, C., Zhebentyayeva, T., Abbott, A.G., et al. (2012). Identification of simple sequence repeat markers tightly linked to plum pox virus resistance in apricot. Mol. Breed. 30, 1017-1026. https://doi.org/10.1007/s11032-011-9685-4

Sosinski, B., Gannavarapu, M., Hager, L.D., Beck, L.E., King, G.J., Ryder, C.D.,et al. (2000). Characterization of microsatellite markers in peach [Prunus persica (L.) Batsch]. Theor. Appl. Genet., 101, 421-428. https://doi.org/10.1007/s001220051499

Sosinski, B., Sossey-Alaoui, K., Rajapakse, S., Glassmoyer, K., Ballard, R.E., Abbott, A.G., et al. (1998). Use of AFLP and RFLP Markers to create a combined linkage map in peach [Prunus persica (L.) Batsch] for use in marker assisted selection. Acta Hortic., 465, 61-68. https://doi.org/10.17660/ActaHortic.1998.465.4

Southon, S., and Faulks, R. (2002). Health benefits of increased fruit and vegetable consumption. Fruit Veg. Process., 5-22. https://doi.org/10.1533/9781855736641.1.5

Stacewicz-Sapuntzakis, M., Bowen, P.E., Hussain, E.A., Damayanti-Wood, B.I., and Farnsworth, N.R. (2001). Chemical composition and potential health effects of prunes: a functional food?, Critical Reviews in Food Science and Nutrition. https://doi.org/10.1080/20014091091814

Tabart, J., Kevers, C., Pincemail, J., Defraigne, J.O., and Dommes, J. (2006). Antioxidant capacity of black currant varies with organ, season, and cultivar. J. Agric. Food Chem., 54, 6271-6276. https://doi.org/10.1021/jf061112y 
Tanksley, S.D., and McCouch, S.R. (1997). Seed banks and molecular maps: unlocking genetic potential from the wild. Science, 277, 1063-1066. https://doi.org/10.1126/science.277.5329.1063

Tieman, D., Zhu, G., Resende, M.F.R., Lin, T., Nguyen, C., Bies, D., et al. (2017). A chemical genetic roadmap to improved tomato flavor. Science, 355, 391-394. https://doi.org/10.1126/SCIENCE.AAL1556

Tuan, P.A., Bai, S., Yaegaki, H., Tamura, T., Hihara, S., Moriguchi, T.,et al. (2015). The crucial role of PpMYB10.1 in anthocyanin accumulation in peach and relationships between its allelic type and skin color phenotype. BMC Plant Biol., 15, 280. https://doi.org/10.1186/s12870-015-0664-5

Van Nocker, S., and Gardiner, S. E. (2014). Breeding better cultivars, faster: applications of new technologies for the rapid deployment of superior horticultural tree crops. Hortic. Res., 1, 14022. https://doi.org/10.1038/hortres.2014.22

Vavilov, N.I. (1951). "The origin, variation, immunity, and breeding of cultivated plants", in Phytogeographic Basis of Plant Breeding, ed. K.S. Chester (Waltham (USA): Chronica Botanica Company), pp. 13, 366.

Veberic, R., and Stampar, F. (2005). Selected polyphenols in fruits of different cultivars of genus Prunus. Phyton; Annales Rei Botanicae, 45, 375-383.

Velasco, R., Zharkikh, A., Affourtit, J., Dhingra, A., Cestaro, A., Kalyanaraman, A., et al. (2010). The genome of the domesticated apple (Malus $x$ domestica Borkh.). Nat. Genet., 42, 833-839. https://doi.org/10.1038/ng.654

Verde, I., Jenkins, J., Dondini, L., Micali, S., Pagliarani, G., Vendramin, E., et al. (2017). The peach v2.0 release: high-resolution linkage mapping and deep resequencing improve chromosome-scale assembly and contiguity. BMC Genomics, 18, 1-18. https://doi.org/10.1186/s12864-017-3606-9

Vieira da Silva, B., Barreira, J.C.M., and Oliveira, M.B.P.P. (2016). Natural phytochemicals and probiotics as bioactive ingredients for functional foods: extraction, biochemistry and protected-delivery technologies. Trends Food Sci. Technol., 50, 144-158. https://doi.org/10.1016/j.tifs.2015.12.007

Vilanova, S., Romero, C., Abbott, A.G., Llácer, G., and Badenes, M.L. (2003). An apricot (Prunus armeniaca L.) F2 progeny linkage map based on SSR and AFLP markers, mapping plum pox virus resistance and self-incompatibility traits. Theor. Appl. Genet. 107, 239-247. https://doi.org/10.1007/s00122-003-1243-y 
Vilanova, S., Romero, C., Llácer, G., Badenes, M., and Burgos, L. (2005). Identification of Self(in)compatibility alleles in apricot by PCR and sequence analysis. Journal of the American Society for Horticultural Science jashs, 130(6), 893-89. https://doi.org/10.21273/JASHS.130.6.893

Voo, S.S., Grimes, H.D., and Lange, B.M. (2012). Assessing the biosynthetic capabilities of secretory glands in Citrus peel. Plant Physiol., 159, 81-94. https://doi.org/10.1104/PP.112.194233

Vu, V. (2011). R Package 'ggbiplot'. A biplot based on 'ggplot2'. https://github.com/vqv/ggbiplot

Wang, Z., Cao, J., and Jiang, W. (2016). Changes in sugar metabolism caused by exogenous oxalic acid related to chilling tolerance of apricot fruit. Postharvest Biol. Technol. 114, 10-16. https://doi.org/10.1016/j.postharvbio.2015.11.015

Wang, K., Jin, P., Han, L., Shang, H., Tang, S., Rui, H., et al. (2014b). Methyl jasmonate induces resistance against Penicillium citrinum in Chinese bayberry by priming of defense responses. Postharvest Biol. Technol., 98, 90-97. https://doi.org/10.1016/j.postharvbio.2014.07.009

Wang, Z., Kang, M., Liu, H., Gao, J., Zhang, Z., Li, Y., et al. (2014a). High-level genetic diversity and complex population structure of Siberian apricot (Prunus sibirica L.) in China as revealed by nuclear SSR markers. PLoS One, 9, e87381. https://doi.org/10.1371/journal.pone.0087381

Wickham, H. (2016a). R package 'readxl'. https://readxl.tidyverse.org

Wickham, H. (2016b). ggplot2: Elegant Graphics for Data Analysis, (New York: Springer-Verlag). https://doi.org/10.1007/978-3-319-24277-4

Wickham, H. (2020). R package 'plyr'. https://github.com/hadley/plyr

Wickham, H. (2021). R package 'dplyr'. https://dplyr.tidyverse.org

Wickham, H. and Seidel, D. (2020). R package 'scales'. https://scales.r-lib.org

Wolfe, K., Wu, X., and Liu, R.H. (2003). Antioxidant activity of apple peels. J. Agric. Food Chem., 51, 609-614. https://doi.org/10.1021/jf020782a

Xi, W., and Lei, Y. (2020). "Apricot", in Nutritional Composition and Antioxidant Properties of Fruits and Vegetables, ed. A. K. Jaiswal (Elsevier), 613-629. https://doi.org/10.1016/B978-0-12-8127803.00038-6

Xi, W., Zheng, H., Zhang, Q., and Li, W. (2016). Profiling taste and aroma compound metabolism during apricot fruit development and ripening. Int. J. Mol. Sci., 17, 998. https://doi.org/10.3390/ijms17070998 
Xiang, Y., Huang, C.H., Hu, Y., Wen, J., Li, S., Yi, T., et al. (2017). Evolution of Rosaceae fruit types based on nuclear phylogeny in the context of geological times and genome duplication. Mol. Biol. Evol., 34, 262-281. https://doi.org/10.1093/molbev/msw242

Xie, D.-Y., Jackson, L.A., Cooper, J.D., Ferreira, D., and Paiva, N.L. (2004). molecular and biochemical analysis of two cDNA clones encoding dihydroflavonol-4-reductase from Medicago truncatula. Plant Physiol., 134, 979-994. https://doi.org/10.1104/pp.103.030221

Yamamoto, T., Yamaguchi, M., and Hayashi, T. (2005). An integrated genetic linkage map of peach by SSR, STS, AFLP and RAPD. J. Japanese Soc. Hortic. Sci., 74, 204-213. https://doi.org/10.2503/jjshs.74.204

Yao, L.H., Jiang, Y.M., Shi, J., Tomás-Barberán, F.A., Datta, N., Singanusong, R., et al. (2004). Flavonoids in food and their health benefits. Plant Foods Hum. Nutr., https://doi.org/10.1007/s11130-0040049-7

You, S., Cao, K., Chen, C., Li, Y., Wu, J., Zhu, G., et al. (2021). Selection and validation reference genes for qRT-PCR normalization in different cultivars during fruit ripening and softening of peach (Prunus persica). Sci. Rep., 11, 7302. https://doi.org/10.1038/s41598-021-86755-5

Zeballos, J.L., Abidi, W., Giménez, R., Monforte, A.J., Moreno, M.Á., and Gogorcena, Y. (2016). Mapping QTLs associated with fruit quality traits in peach [Prunus persica (L.) Batsch] using SNP maps. Tree Genetics \& Genomes 12, 37. https://doi.org/10.1007/s11295-016-0996-9

Zhang, Q., Feng, C., Li, W., Qu, Z., Zeng, M., and Xi, W. (2019). Transcriptional regulatory networks controlling taste and aroma quality of apricot (Prunus armeniaca L.) fruit during ripening. BMC Genomics, 20, 1-15. https://doi.org/10.1186/s12864-019-5424-8

Zhang, C., Shen, Z., Zhang, Y., Han, J., Ma, R., Korir, N.K., et al. (2013). Cloning and expression of genes related to the sucrose-metabolizing enzymes and carbohydrate changes in peach. Acta Physiol. Plant., 35, 589-602. https://doi.org/10.1007/s11738-012-1100-1

Zhebentyayeva, T., Ledbetter, C., Burgos, L., and Llácer, G. (2012). "Apricot", in Fruit Breeding Handbook of Plant Breeding vol. 8, eds. M. L. Badenes and D.H. Byrne. (Boston (MA): Springer), 415-458. https://doi.org/10.1007/978-1-4419-0763-9_12

Zuriaga, E., Romero, C., Blanca, J.M., and Badenes, M.L. (2018). Resistance to Plum Pox Virus (PPV) in apricot (Prunus armeniaca L.) is associated with down-regulation of two MATHd genes. BMC Plant Biol., 18, 25. https://doi.org/10.1186/s12870-018-1237-1 
ANNEX I. Supplementary Figures 
A

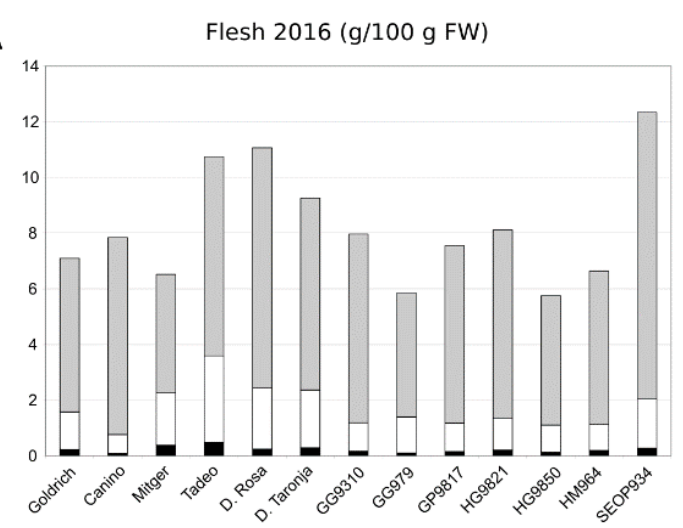

C

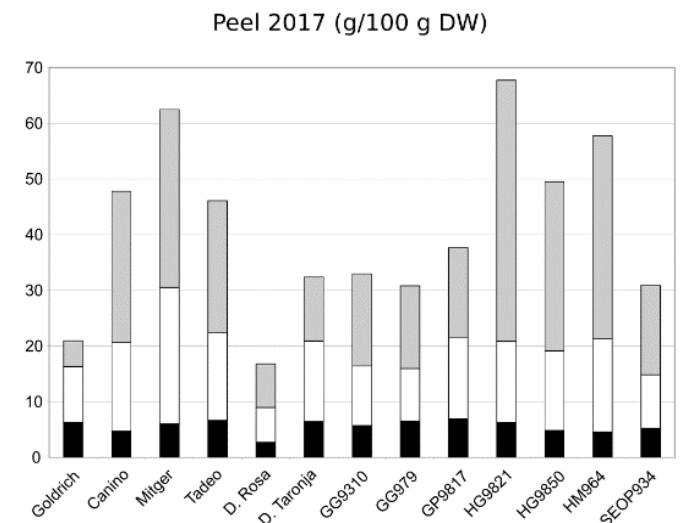

B

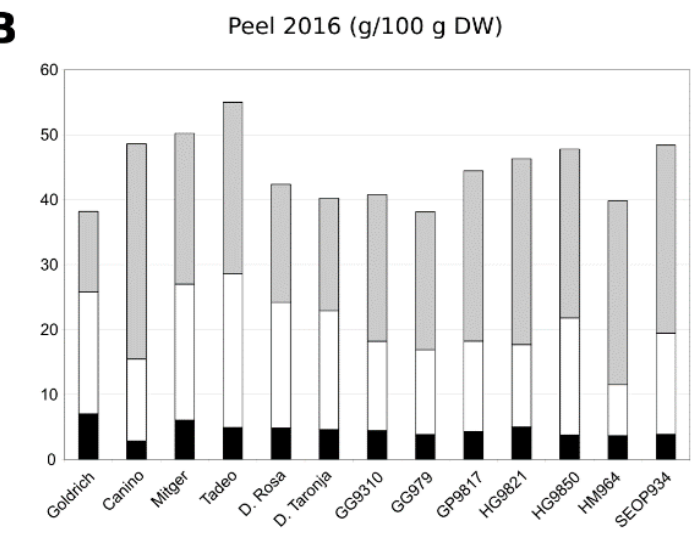

D

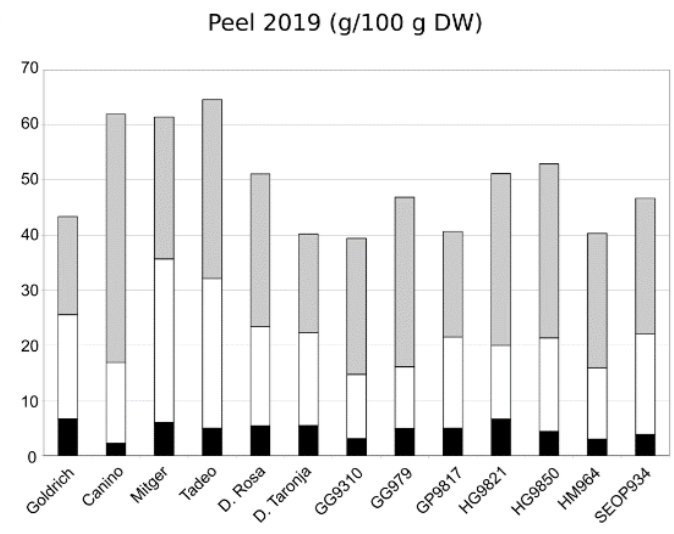

Figure S1. Profiles of sugar content in flesh (g/100 g fresh weight (FW)) and peel (g /100 g dry weight (DW)) during 2016, 2017 and 2019. 
A

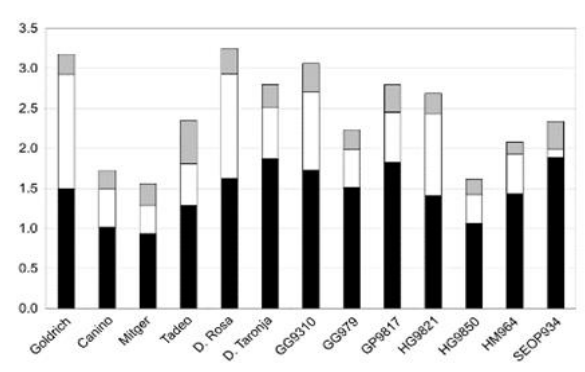

Flesh 2016 (g/100 g FW)

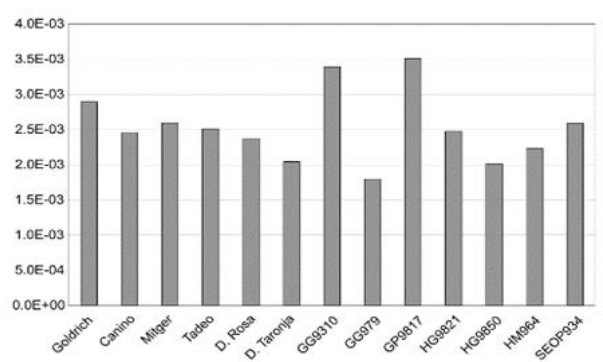

Peel 2016 (g/100 g DW)

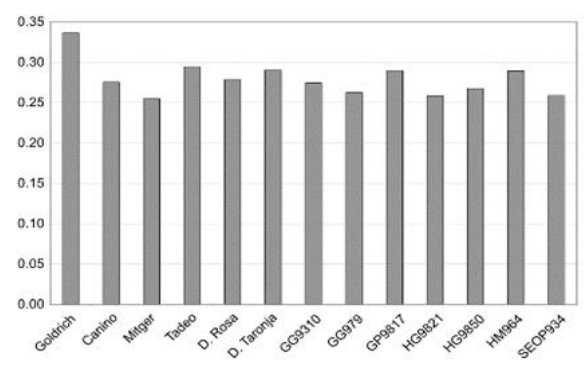

C

Peel 2017 (g/100 g DW)
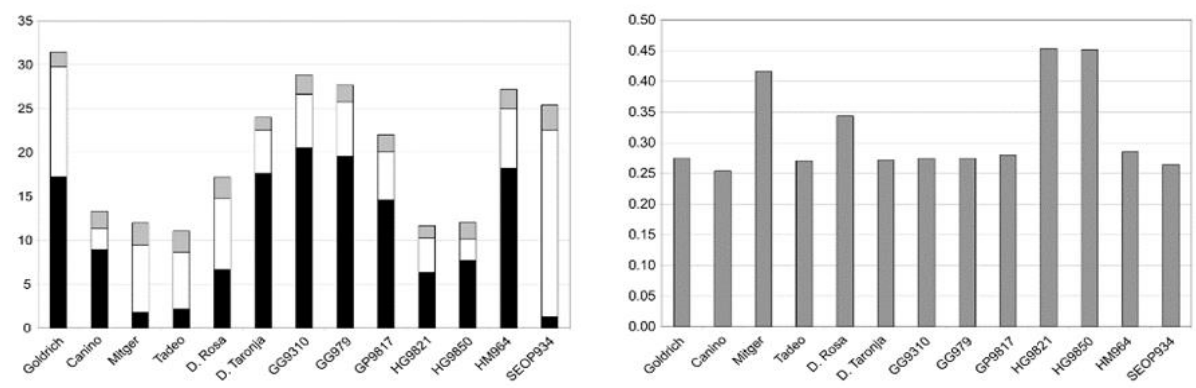

D

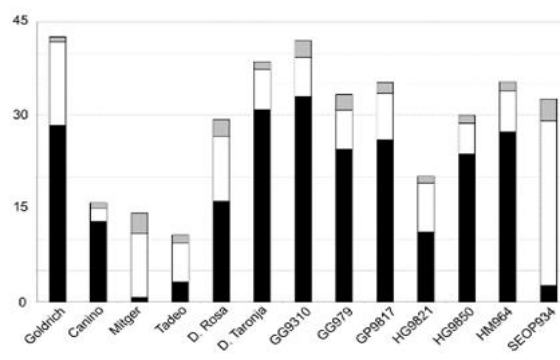

- Citric 口Malic 口Succinic

Peel 2019 (g/100 g DW)

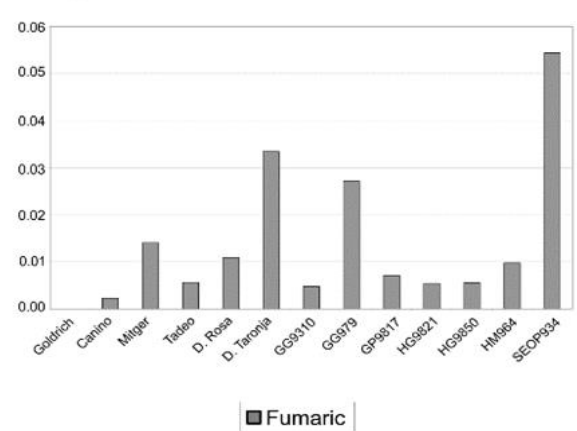

Figure S2. Profiles of organic acids content in flesh (g/100 g FW) and peel (g /100 g DW) during 2016, 2017 and 2019. 
A

Flesh 2016 (mg/100 g FW)

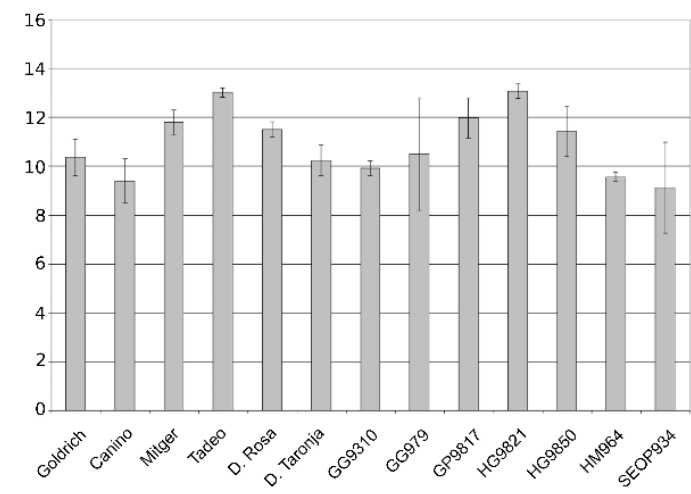

C

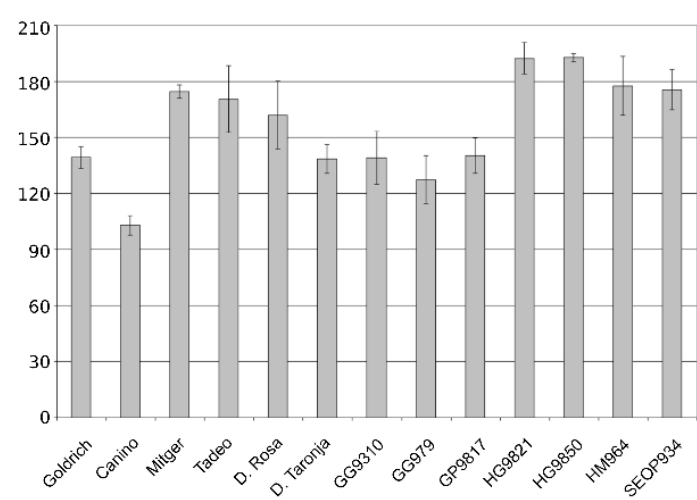

B Peel $2016(\mathrm{mg} / 100 \mathrm{~g} \mathrm{DW})$

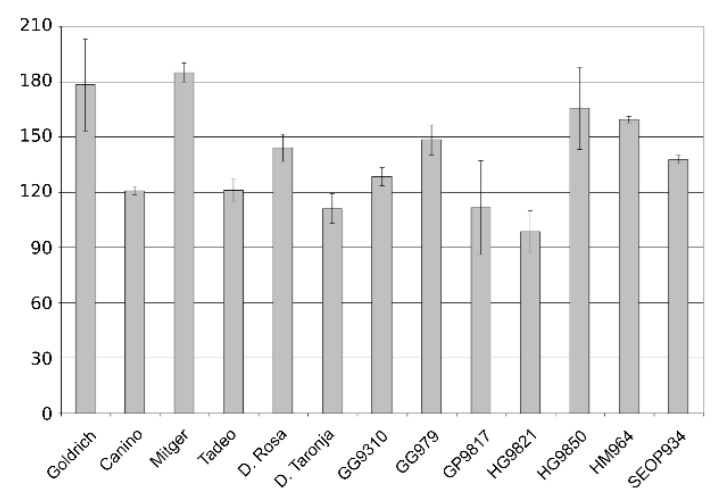

D Peel $2019(\mathrm{mg} / 100 \mathrm{~g} \mathrm{DW})$

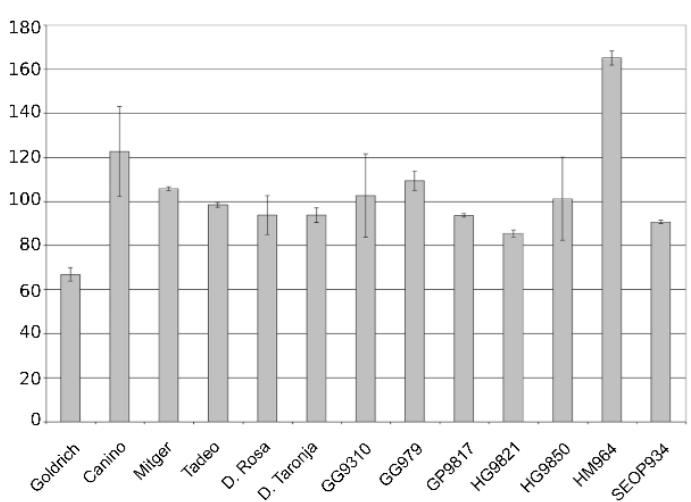

Figure S3. Ascorbic acid content in flesh (mg/100 g FW) and peel (mg /100 g DW) during 2016, 2017 and 2019. 
ANNEX II. Supplementary Tables 
Table S1. Spearman's rank correlation coefficients (upper triangular matrix) and significance levels (lower triangular matrix) among variables analyzed.

\begin{tabular}{|c|c|c|c|c|c|c|c|c|c|c|}
\hline & $\begin{array}{c}\text { Citric } \\
\text { F_2016 }\end{array}$ & $\begin{array}{c}\text { Malic } \\
\text { F_2016 }\end{array}$ & $\begin{array}{l}\text { Succinic } \\
\text { F_2016 }\end{array}$ & $\begin{array}{c}\text { Fumaric } \\
\text { F_2016 }\end{array}$ & $\begin{array}{c}\text { Citric } \\
\text { P_2016 }\end{array}$ & $\begin{array}{c}\text { Malic } \\
\text { P_2016 }\end{array}$ & $\begin{array}{l}\text { Succinic } \\
\text { P_2016 }\end{array}$ & $\begin{array}{l}\text { Fumaric } \\
\text { P_2016 }\end{array}$ & $\begin{array}{c}\text { Citric } \\
\text { P_2017 }\end{array}$ & $\begin{array}{c}\text { Malic } \\
\text { P_2017 }\end{array}$ \\
\hline Citric_F_2016 & 1 & 0,22 & 0,51 & 0,19 & 0,32 & 0,09 & $-0,12$ & 0,13 & 0,23 & 0,22 \\
\hline Malic_F_2016 & 0,51 & 1 & 0,26 & 0,03 & 0,48 & $-0,02$ & $-0,56$ & 0,78 & 0,55 & 0,05 \\
\hline Succinic_F_2016 & 0,39 & 0,99 & 1 & 0,64 & $-0,17$ & 0,03 & 0,32 & 0,28 & $-0,10$ & 0,35 \\
\hline Fumaric_F2016 & 0,42 & 0,99 & 0,15 & 1 & $-0,27$ & 0,24 & 0,17 & 0,15 & $-0,17$ & 0,39 \\
\hline Citric_P_2016 & 0,42 & 0,17 & 0,16 & 0,46 & 1 & $-0,29$ & $-0,92$ & 0,31 & 0,96 & $-0,40$ \\
\hline Malic_P_2016 & 0,29 & 0,74 & 0,82 & 0,47 & 0,15 & 1 & 0,13 & $-0,29$ & $-0,28$ & 0,83 \\
\hline Succinic_P_2016 & 0,79 & 0,04 & 0,13 & 0,74 & 0,00 & 0,31 & 1 & $-0,36$ & $-0,91$ & 0,27 \\
\hline Fumaric_P_2016 & 0,57 & 0,02 & 0,66 & 0,47 & 0,29 & 0,82 & 0,06 & 1 & 0,28 & $-0,06$ \\
\hline Citric_P_2017 & 0,20 & 0,16 & 0,42 & 0,86 & 0,00 & 0,29 & 0,00 & 0,27 & 1 & $-0,42$ \\
\hline Malic_P_2017 & 0,18 & 0,90 & 0,59 & 0,33 & 0,13 & $1,97 \cdot 10^{8}$ & 0,41 & 0,91 & 0,37 & 1 \\
\hline Succinic_P_2017 & 0,91 & 0,29 & 0,17 & 0,37 & 0,00 & 0,05 & 0,00 & 0,09 & 0,01 & 0,08 \\
\hline Fumaric_P_2017 & 0,08 & 0,66 & 0,22 & 0,57 & 0,49 & 0,46 & 0,67 & 0,27 & 0,25 & 0,46 \\
\hline Citric_P_2019 & 0,20 & 0,11 & 0,31 & 0,75 & $1,93 \cdot 10^{9}$ & 0,21 & 0,00 & 0,22 & $4,57 \cdot 10$ & 0,27 \\
\hline Malic_P_2019 & 0,19 & 0,72 & 0,74 & 0,35 & 0,10 & $7,17 \cdot 10^{8}$ & 0,30 & 0,86 & 0,26 & $9,95 \cdot 10^{6}$ \\
\hline Succinic_P_2019 & 0,48 & 0,46 & 0,72 & 0,60 & 0,12 & 0,09 & 0,13 & 0,02 & 0,36 & 0,10 \\
\hline Fumaric_P_2019 & 0,11 & 0,10 & 0,93 & 0,75 & 0,37 & 0,05 & 0,24 & 0,18 & 0,61 & 0,04 \\
\hline Ascorbic_F_2016 & 0,39 & 0,88 & 0,16 & 0,41 & 0,39 & 0,14 & 0,54 & 0,73 & 0,35 & 0,28 \\
\hline Ascorbic_P_2016 & 0,12 & 0,69 & 0,14 & 0,82 & 0,51 & 0,58 & 0,52 & 0,97 & 0,56 & 0,52 \\
\hline Ascorbic_P_2017 & 0,69 & 0,44 & 0,58 & 0,72 & 0,04 & 0,53 & 0,09 & 0,45 & 0,04 & 0,39 \\
\hline Ascorbic_P_2019 & 0,15 & 0,08 & 0,72 & 0,26 & 0,92 & 0,24 & 0,32 & 0,01 & 0,68 & 0,08 \\
\hline Fructose_F_2016 & 0,89 & 0,82 & 0,02 & 0,41 & 0,01 & 0,70 & 0,15 & 0,74 & 0,08 & 0,36 \\
\hline Glucose_F_2016 & 0,80 & 0,97 & 0,01 & 0,95 & 0,05 & 0,84 & 0,16 & 0,79 & 0,14 & 0,49 \\
\hline Sucrose_F_2016 & 0,08 & 1,00 & 0,17 & 0,83 & 0,34 & 0,04 & 0,09 & 0,91 & 0,32 & 0,07 \\
\hline Fructose_P_2016 & 0,95 & 0,08 & 0,74 & 0,28 & 0,62 & 0,79 & 0,28 & 0,07 & 0,95 & 0,39 \\
\hline Glucose_P_2016 & 0,41 & 0,72 & 0,19 & 0,91 & 0,07 & 0,78 & 0,42 & 0,46 & 0,09 & 0,83 \\
\hline Sucrose_P_2016 & 0,35 & 0,01 & 0,72 & 0,80 & 0,28 & 0,82 & 0,02 & 0,07 & 0,13 & 0,75 \\
\hline Fructose_P_2017 & 0,70 & 0,48 & 0,47 & 0,11 & 0,75 & 0,58 & 0,42 & 0,42 & 0,29 & 0,89 \\
\hline Glucose_P_2017 & 0,04 & 0,13 & 0,95 & 0,51 & 0,25 & 0,27 & 0,53 & 0,50 & 0,26 & 0,31 \\
\hline Sucrose_P_2017 & 0,01 & 0,02 & 0,80 & 0,90 & 0,19 & 0,38 & 0,14 & 0,06 & 0,10 & 0,23 \\
\hline Fructose_P_2019 & 0,86 & 0,26 & 0,97 & 0,63 & 0,81 & 0,91 & 0,34 & 0,17 & 0,96 & 0,61 \\
\hline Glucose_P_2019 & 0,18 & 0,62 & 0,24 & 0,37 & 0,00 & 0,91 & 0,17 & 0,91 & 0,01 & 0,66 \\
\hline Sucrose_P_2019 & 0,03 & 0,30 & 0,79 & 0,14 & 0,67 & 0,61 & 0,31 & 0,22 & 0,30 & 0,25 \\
\hline Weight_2016 & 0,44 & 0,87 & 0,04 & 0,15 & 0,01 & 0,29 & 0,09 & 0,81 & 0,03 & 0,29 \\
\hline Stone.weight_2016 & 0,73 & 0,07 & 0,99 & 0,79 & 0,81 & 0,98 & 0,22 & 0,03 & 0,87 & 0,87 \\
\hline Height_2016 & 0,65 & 0,62 & 0,05 & 0,15 & 0,01 & 0,19 & 0,02 & 0,56 & 0,02 & 0,21 \\
\hline LateralWidth_2016 & 0,23 & 0,93 & 0,04 & 0,32 & 0,02 & 0,61 & 0,15 & 0,60 & 0,04 & 0,54 \\
\hline VentralWidth_2016 & 0,47 & 0,27 & 0,07 & 0,43 & 0,20 & 0,66 & 0,41 & 0,19 & 0,16 & 0,69 \\
\hline Firmness_2016 & 0,09 & 0,88 & 0,95 & 0,51 & 0,75 & 0,30 & 0,90 & 0,30 & 0,46 & 0,30 \\
\hline Weight_2017 & 0,25 & 0,77 & 0,07 & 0,26 & 0,54 & 0,10 & 0,49 & 0,99 & 0,62 & 0,11 \\
\hline Stone.weight_2017 & 0,85 & 0,44 & 0,12 & 0,50 & 0,04 & 0,19 & 0,07 & 0,57 & 0,12 & 0,17 \\
\hline Height_2017 & 0,46 & 0,20 & 0,11 & 0,35 & 0,30 & 0,64 & 0,06 & 0,19 & 0,30 & 0,44 \\
\hline LateralWidth_2017 & 0,14 & 0,91 & 0,33 & 0,81 & 0,89 & 0,11 & 0,91 & 0,84 & 0,83 & 0,07 \\
\hline VentralWidth_2017 & 0,45 & 0,46 & 0,19 & 0,66 & 0,51 & 0,04 & 0,70 & 0,43 & 0,53 & 0,03 \\
\hline Firmness_2017 & 0,02 & 0,70 & 0,33 & 0,97 & 0,82 & 0,51 & 0,88 & 0,79 & 0,28 & 0,36 \\
\hline Weight_2019 & 0,56 & 0,66 & 0,14 & 0,44 & 0,24 & 0,25 & 0,02 & 0,23 & 0,21 & 0,43 \\
\hline Stone.weight_2019 & 0,00 & 0,82 & 0,64 & 0,75 & 0,37 & 0,45 & 0,73 & 0,87 & 0,54 & 0,53 \\
\hline Height_2019 & 0,58 & 0,31 & 0,20 & 0,50 & 0,17 & 0,22 & 0,01 & 0,07 & 0,15 & 0,41 \\
\hline LateralWidth_2019 & 0,43 & 0,66 & 0,19 & 0,80 & 0,22 & 0,13 & 0,02 & 0,20 & 0,18 & 0,27 \\
\hline VentralWidth_2019 & 0,28 & 0,69 & 0,24 & 0,73 & 0,71 & 0,24 & 0,17 & 0,73 & 0,52 & 0,43 \\
\hline Firmness_2019 & 0,49 & 0,66 & 0,43 & 0,00 & 0,93 & 0,59 & 0,83 & 0,38 & 0,81 & 0,83 \\
\hline
\end{tabular}


Table S1. (Cont.)

\begin{tabular}{|c|c|c|c|c|c|c|c|c|c|c|}
\hline & $\begin{array}{l}\text { Fumaric } \\
\text { P_2017 }\end{array}$ & $\begin{array}{c}\text { Citric } \\
\text { P_2019 }\end{array}$ & $\begin{array}{c}\text { Malic } \\
\text { P_2019 }\end{array}$ & $\begin{array}{l}\text { Succinic } \\
\text { P_2019 }\end{array}$ & $\begin{array}{l}\text { Fumaric } \\
\text { P_2019 }\end{array}$ & $\begin{array}{c}\text { Ascorbic } \\
\text { F_2016 }\end{array}$ & $\begin{array}{c}\text { Ascorbic } \\
\text { P_2016 }\end{array}$ & $\begin{array}{c}\text { Ascorbic } \\
\text { P_2017 }\end{array}$ & $\begin{array}{c}\text { Ascorbic } \\
\text { P_2019 }\end{array}$ & $\begin{array}{c}\text { Fructose } \\
\text { F_2016 }\end{array}$ \\
\hline Citric_F_2016 & $-0,26$ & 0,43 & 0,46 & 0,26 & 0,50 & $-0,33$ & $-0,50$ & $-0,07$ & $-0,58$ & 0,13 \\
\hline Malic_F_2016 & 0,04 & 0,69 & 0,12 & $-0,42$ & $-0,48$ & 0,07 & $-0,21$ & $-0,43$ & $-0,36$ & 0,01 \\
\hline Succinic_F_2016 & $-0,21$ & 0,06 & 0,24 & 0,41 & 0,13 & 0,25 & $-0,43$ & 0,16 & $-0,37$ & 0,57 \\
\hline Fumaric_F2016 & 0,01 & $-0,04$ & 0,38 & 0,25 & $-0,13$ & 0,27 & $-0,02$ & 0,30 & $-0,44$ & 0,34 \\
\hline Citric_P_2016 & $-0,11$ & 0,87 & $-0,23$ & $-0,47$ & $-0,13$ & $-0,27$ & $-0,30$ & $-0,76$ & 0,12 & $-0,50$ \\
\hline Malic_P_2016 & $-0,03$ & $-0,30$ & 0,74 & 0,48 & 0,09 & $-0,27$ & 0,56 & 0,14 & $-0,31$ & 0,07 \\
\hline Succinic_P_2016 & $-0,05$ & $-0,84$ & 0,07 & 0,57 & 0,30 & 0,20 & 0,05 & 0,71 & $-0,03$ & 0,47 \\
\hline Fumaric_P_2016 & $-0,21$ & 0,46 & $-0,03$ & $-0,68$ & $-0,48$ & 0,26 & $-0,42$ & $-0,28$ & $-0,47$ & 0,13 \\
\hline Citric_P_2017 & $-0,02$ & 0,91 & $-0,28$ & $-0,43$ & $-0,27$ & $-0,24$ & $-0,25$ & $-0,75$ & 0,19 & $-0,49$ \\
\hline Malic_P_2017 & 0,01 & $-0,32$ & 0,84 & 0,51 & 0,25 & 0,05 & 0,49 & 0,39 & $-0,56$ & 0,54 \\
\hline Succinic_P_2017 & $-0,06$ & $-0,71$ & 0,28 & 0,81 & 0,27 & 0,16 & 0,13 & 0,50 & $-0,01$ & 0,45 \\
\hline Fumaric_P_2017 & 1 & 0,07 & 0,12 & 0,22 & 0,00 & 0,55 & 0,56 & 0,45 & $-0,02$ & $-0,04$ \\
\hline Citric_P_2019 & 0,62 & 1 & $-0,10$ & $-0,44$ & $-0,27$ & $-0,20$ & $-0,30$ & $-0,52$ & $-0,16$ & $-0,32$ \\
\hline Malic_P_2019 & 0,74 & 0,24 & 1 & 0,44 & 0,42 & $-0,06$ & 0,31 & 0,29 & $-0,73$ & 0,43 \\
\hline Succinic_P_2019 & 0,61 & 0,23 & 0,06 & 1 & 0,61 & 0,06 & 0,24 & 0,45 & 0,00 & 0,32 \\
\hline Fumaric_P_2019 & 0,56 & 0,46 & 0,02 & 0,08 & 1 & $-0,04$ & $-0,09$ & 0,21 & $-0,10$ & 0,36 \\
\hline Ascorbic_F_2016 & 0,21 & 0,50 & 0,32 & 0,76 & 0,19 & 1 & 0,14 & 0,36 & $-0,05$ & 0,32 \\
\hline Ascorbic_P_2016 & 0,03 & 0,60 & 0,49 & 0,55 & 0,68 & 0,81 & 1 & 0,43 & 0,04 & 0,03 \\
\hline Ascorbic_P_2017 & 0,02 & 0,20 & 0,23 & 0,25 & 0,57 & 0,16 & 0,20 & 1 & $-0,37$ & 0,46 \\
\hline Ascorbic_P_2019 & 0,78 & 0,48 & 0,10 & 0,78 & 0,89 & 0,85 & 0,43 & 0,44 & 1 & $-0,37$ \\
\hline Fructose_F_2016 & 0,86 & 0,06 & 0,39 & 0,59 & 0,65 & 0,11 & 0,83 & 0,10 & 0,40 & 1 \\
\hline Glucose_F_2016 & 0,93 & 0,12 & 0,54 & 0,64 & 0,50 & 0,10 & 0,82 & 0,17 & 0,49 & $1,52 \mathrm{E}-$ \\
\hline Sucrose_F_2016 & 0,15 & 0,40 & 0,07 & 0,39 & 0,15 & 0,32 & 0,11 & 0,89 & 0,66 & 0,59 \\
\hline Fructose_P_2016 & 0,65 & 0,99 & 0,48 & 1,00 & 0,49 & 0,33 & 0,06 & 0,49 & 0,01 & 0,09 \\
\hline Glucose_P_2016 & 0,25 & 0,18 & 0,82 & 0,86 & 0,71 & 0,03 & 0,34 & 0,04 & 0,28 & 0,00 \\
\hline Sucrose_P_2016 & 0,94 & 0,12 & 0,85 & 0,81 & 0,82 & 0,81 & 0,27 & 0,98 & 0,02 & 0,67 \\
\hline Fructose_P_2017 & 0,32 & 0,61 & 0,76 & 0,49 & 0,89 & 0,63 & 0,68 & 0,59 & 0,66 & 0,56 \\
\hline Glucose_P_2017 & 0,24 & 0,21 & 0,40 & 0,80 & 0,57 & 0,28 & 0,62 & 0,66 & 0,27 & 0,28 \\
\hline Sucrose_P_2017 & 0,10 & 0,12 & 0,34 & 0,95 & 0,61 & 0,46 & 0,67 & 0,36 & 0,02 & 0,80 \\
\hline Fructose_P_2019 & 0,43 & 0,93 & 0,60 & 0,96 & 0,95 & 0,12 & 0,12 & 0,34 & 0,03 & 0,15 \\
\hline Glucose_P_2019 & 0,26 & 0,01 & 0,60 & 0,77 & 0,82 & 0,04 & 0,29 & 0,07 & 0,66 & $7,68 \cdot 10^{-4}$ \\
\hline Sucrose_P_2019 & 0,88 & 0,23 & 0,22 & 0,64 & 0,48 & 0,85 & 0,79 & 0,57 & 0,00 & 0,47 \\
\hline Weight_2016 & 0,94 & 0,02 & 0,37 & 0,50 & 0,47 & 0,18 & 0,61 & 0,23 & 0,69 & 0,07 \\
\hline Stone.weight_2016 & 0,44 & 0,88 & 0,93 & 0,25 & 0,27 & 0,56 & 0,56 & 0,26 & 0,24 & 0,71 \\
\hline Height_2016 & 0,83 & 0,01 & 0,24 & 0,15 & 0,95 & 0,21 & 0,70 & 0,13 & 0,97 & 0,14 \\
\hline LateralWidth_2016 & 0,79 & 0,04 & 0,67 & 0,99 & 0,33 & 0,14 & 0,55 & 0,16 & 0,68 & 0,03 \\
\hline VentralWidth_2016 & 0,71 & 0,20 & 0,87 & 0,57 & 0,13 & 0,24 & 0,97 & 0,67 & 0,51 & 0,19 \\
\hline Firmness_2016 & 0,88 & 0,42 & 0,26 & 0,02 & 0,36 & 0,64 & 0,89 & 0,49 & 0,68 & 0,40 \\
\hline Weight_2017 & 0,81 & 0,41 & 0,08 & 0,58 & 0,08 & 0,04 & 0,36 & 0,70 & 0,20 & 0,35 \\
\hline Stone.weight_2017 & 0,63 & 0,16 & 0,19 & 0,26 & 0,65 & 0,48 & 0,54 & 0,02 & 0,68 & 0,20 \\
\hline Height_2017 & 0,73 & 0,17 & 0,41 & 0,61 & 0,55 & 0,29 & 0,20 & 0,89 & 0,03 & 0,83 \\
\hline LateralWidth_2017 & 0,40 & 0,74 & 0,02 & 0,26 & 0,06 & 0,40 & 0,43 & 0,15 & 0,09 & 0,96 \\
\hline VentralWidth_2017 & 0,38 & 0,73 & 0,01 & 0,16 & 0,01 & 0,19 & 0,21 & 0,16 & 0,30 & 0,99 \\
\hline Firmness_2017 & 0,05 & 0,70 & 0,48 & 0,18 & 0,10 & 0,32 & 0,24 & 0,27 & 0,92 & 0,65 \\
\hline Weight_2019 & 0,72 & 0,22 & 0,41 & 0,11 & 0,74 & 0,75 & 0,34 & 0,56 & 0,52 & 0,98 \\
\hline Stone.weight_2019 & 0,09 & 0,38 & 0,50 & 1,00 & 0,18 & 0,26 & 0,01 & 0,48 & 0,72 & 0,41 \\
\hline Height_2019 & 0,89 & 0,17 & 0,34 & 0,05 & 0,45 & 0,89 & 0,34 & 0,41 & 0,35 & 1,00 \\
\hline LateralWidth_2019 & 0,57 & 0,19 & 0,26 & 0,09 & 0,41 & 0,89 & 0,31 & 0,62 & 0,54 & 0,99 \\
\hline VentralWidth_2019 & 0,42 & 0,65 & 0,44 & 0,39 & 0,88 & 0,94 & 0,23 & 0,90 & 0,95 & 0,65 \\
\hline Firmness_2019 & 0,76 & 0,62 & 0,94 & 0,94 & 0,36 & 0,08 & 0,79 & 0,61 & 0,26 & 0,67 \\
\hline
\end{tabular}


Table S1. (Cont.)

\begin{tabular}{|c|c|c|c|c|c|c|c|c|c|c|}
\hline & $\begin{array}{l}\text { Glucose } \\
\text { F_2016 } \\
\end{array}$ & $\begin{array}{l}\text { Sucrose } \\
\text { F_2016 } \\
\end{array}$ & $\begin{array}{c}\text { Fructose } \\
\text { P_2016 } \\
\end{array}$ & $\begin{array}{l}\text { Glucose } \\
\text { P_2016 }\end{array}$ & $\begin{array}{l}\text { Sucrose } \\
\text { P_2016 }\end{array}$ & $\begin{array}{c}\text { Fructose } \\
\text { P_2017 }\end{array}$ & $\begin{array}{l}\text { Glucose } \\
\text { P_2017 }\end{array}$ & $\begin{array}{c}\text { SucroseP } \\
2017 \\
\end{array}$ & $\begin{array}{c}\text { Fructose } \\
\text { P_2019 } \\
\end{array}$ & $\begin{array}{l}\text { Glucose } \\
\text { P_2019 } \\
\end{array}$ \\
\hline Citric_F_2016 & 0,19 & 0,47 & $-0,06$ & $-0,20$ & $-0,17$ & 0,20 & $-0,52$ & $-0,61$ & $-0,07$ & $-0,28$ \\
\hline Malic_F_2016 & 0,16 & 0,16 & 0,46 & 0,14 & $-0,64$ & 0,07 & $-0,27$ & $-0,64$ & 0,36 & $-0,13$ \\
\hline Succinic_F_2016 & 0,50 & 0,49 & 0,41 & 0,29 & 0,12 & 0,38 & 0,01 & $-0,12$ & 0,03 & 0,17 \\
\hline Fumaric_F2016 & 0,08 & $-0,03$ & 0,42 & 0,19 & 0,21 & 0,50 & 0,26 & 0,10 & 0,17 & 0,34 \\
\hline Citric_P_2016 & $-0,33$ & $-0,27$ & $-0,21$ & $-0,55$ & $-0,52$ & 0,33 & $-0,30$ & $-0,47$ & $-0,06$ & $-0,76$ \\
\hline Malic_P_2016 & 0,15 & 0,12 & 0,27 & 0,05 & $-0,12$ & $-0,31$ & $-0,49$ & $-0,36$ & 0,12 & 0,27 \\
\hline Succinic_P_2016 & 0,34 & 0,46 & $-0,01$ & 0,40 & 0,66 & $-0,30$ & 0,23 & 0,49 & $-0,20$ & 0,56 \\
\hline Fumaric_P_2016 & 0,23 & 0,26 & 0,40 & 0,27 & $-0,29$ & 0,36 & 0,04 & $-0,48$ & 0,37 & 0,10 \\
\hline Citric_P_2017 & $-0,38$ & $-0,34$ & $-0,14$ & $-0,53$ & $-0,52$ & 0,28 & $-0,22$ & $-0,36$ & $-0,08$ & $-0,76$ \\
\hline Malic_P_2017 & 0,60 & 0,21 & 0,61 & 0,49 & $-0,24$ & 0,00 & $-0,46$ & $-0,44$ & 0,42 & 0,59 \\
\hline Succinic_P_2017 & 0,34 & 0,36 & 0,16 & 0,26 & 0,53 & $-0,15$ & 0,09 & 0,35 & $-0,18$ & 0,42 \\
\hline Fumaric_P_2017 & $-0,05$ & $-0,61$ & 0,20 & 0,30 & $-0,39$ & $-0,07$ & $-0,09$ & 0,16 & 0,39 & 0,15 \\
\hline Citric_P_2019 & $-0,27$ & $-0,18$ & 0,01 & $-0,34$ & $-0,60$ & 0,26 & $-0,29$ & $-0,48$ & 0,05 & $-0,58$ \\
\hline Malic_P_2019 & 0,46 & 0,14 & 0,53 & 0,35 & $-0,37$ & 0,03 & $-0,45$ & $-0,58$ & 0,55 & 0,45 \\
\hline Succinic_P_2019 & 0,30 & 0,14 & 0,06 & 0,11 & 0,17 & $-0,16$ & $-0,26$ & 0,14 & $-0,11$ & 0,15 \\
\hline Fumaric_P_2019 & 0,44 & 0,12 & $-0,08$ & 0,09 & $-0,03$ & 0,13 & $-0,23$ & $-0,15$ & 0,12 & 0,05 \\
\hline Ascorbic_F_2016 & 0,38 & $-0,29$ & 0,42 & 0,59 & $-0,06$ & 0,46 & 0,27 & 0,20 & 0,50 & 0,38 \\
\hline Ascorbic_P_2016 & 0,02 & $-0,54$ & 0,29 & 0,31 & $-0,29$ & $-0,26$ & $-0,24$ & 0,09 & 0,32 & 0,42 \\
\hline Ascorbic_P_2017 & 0,26 & 0,05 & 0,19 & 0,57 & 0,24 & $-0,16$ & 0,01 & 0,33 & 0,15 & 0,68 \\
\hline Ascorbic_P_2019 & $-0,36$ & $-0,36$ & $-0,44$ & $-0,38$ & 0,26 & $-0,16$ & 0,38 & 0,62 & $-0,44$ & $-0,42$ \\
\hline Fructose_F_2016 & 0,88 & 0,26 & 0,76 & 0,85 & $-0,14$ & 0,23 & 0,18 & $-0,05$ & 0,56 & 0,77 \\
\hline Glucose_F_2016 & 1 & 0,34 & 0,72 & 0,81 & $-0,31$ & 0,21 & $-0,12$ & $-0,34$ & 0,61 & 0,62 \\
\hline Sucrose_F_2016 & 0,38 & 1 & $-0,06$ & 0,06 & 0,33 & $-0,31$ & $-0,22$ & $-0,28$ & $-0,30$ & 0,09 \\
\hline Fructose_P_2016 & 0,27 & 0,43 & 1 & 0,81 & $-0,54$ & 0,28 & 0,06 & $-0,30$ & 0,83 & 0,69 \\
\hline Glucose_P_2016 & 0,00 & 0,88 & 0,04 & 1 & $-0,27$ & 0,11 & 0,14 & $-0,04$ & 0,73 & 0,88 \\
\hline Sucrose_P_2016 & 0,52 & 0,48 & 0,01 & 0,35 & 1 & $-0,11$ & 0,46 & 0,66 & $-0,67$ & 0,01 \\
\hline Fructose_P_2017 & 0,92 & 0,20 & 0,50 & 0,98 & 0,81 & 1 & 0,24 & $-0,11$ & 0,35 & $-0,01$ \\
\hline Glucose_P_2017 & 0,84 & 0,14 & 0,69 & 0,35 & 0,32 & 0,25 & 1 & 0,73 & 0,02 & 0,25 \\
\hline Sucrose_P_2017 & 0,77 & 0,29 & 0,32 & 0,74 & 0,02 & 0,92 & 0,01 & 1 & $-0,39$ & 0,11 \\
\hline Fructose_P_2019 & 0,16 & 0,30 & 0,00 & 0,04 & 0,01 & 0,47 & 0,81 & 0,27 & 1 & 0,59 \\
\hline Glucose_P_2019 & 0,03 & 0,81 & 0,08 & 0,00 & 0,97 & 0,72 & 0,03 & 0,24 & 0,13 & 1 \\
\hline Sucrose_P_2019 & 0,65 & 0,98 & 0,05 & 0,61 & 0,02 & 0,26 & 0,67 & 0,06 & 0,03 & 0,86 \\
\hline Weight_2016 & 0,15 & 0,40 & 0,36 & 0,12 & 0,47 & 0,69 & 0,75 & 0,58 & 0,88 & 0,04 \\
\hline Stone.weight_2016 & 0,62 & 0,75 & 0,08 & 0,48 & 0,19 & 0,87 & 0,88 & 0,20 & 0,09 & 0,46 \\
\hline Height_2016 & 0,21 & 0,36 & 0,77 & 0,30 & 0,26 & 0,72 & 0,91 & 0,47 & 0,91 & 0,11 \\
\hline LateralWidth_2016 & 0,09 & 0,67 & 0,29 & 0,03 & 0,58 & 0,93 & 0,52 & 0,38 & 0,84 & 0,02 \\
\hline VentralWidth_2016 & 0,21 & 0,34 & 0,39 & 0,13 & 0,78 & 0,48 & 0,84 & 0,96 & 0,94 & 0,17 \\
\hline Firmness_2016 & 0,53 & 0,62 & 0,74 & 0,24 & 0,76 & 0,72 & 0,09 & 0,38 & 0,77 & 0,16 \\
\hline Weight_2017 & 0,48 & 0,51 & 0,95 & 0,52 & 0,26 & 0,34 & 0,09 & 0,16 & 0,72 & 0,23 \\
\hline Stone.weight_2017 & 0,26 & 0,49 & 0,92 & 0,28 & 0,48 & 0,91 & 0,70 & 0,51 & 0,81 & 0,36 \\
\hline Height_2017 & 0,86 & 0,77 & 0,15 & 0,72 & 0,01 & 0,84 & 0,38 & 0,09 & 0,10 & 0,68 \\
\hline LateralWidth_2017 & 0,97 & 0,34 & 0,65 & 0,89 & 0,28 & 0,34 & 0,27 & 0,23 & 0,33 & 0,79 \\
\hline VentralWidth_2017 & 0,92 & 0,51 & 0,81 & 1,00 & 0,61 & 0,52 & 0,62 & 0,66 & 0,51 & 1,00 \\
\hline Firmness_2017 & 0,41 & 0,27 & 0,92 & 0,56 & 0,38 & 0,63 & 0,33 & 0,08 & 0,96 & 0,47 \\
\hline Weight_2019 & 0,76 & 0,05 & 0,20 & 0,62 & 0,09 & 0,21 & 0,32 & 0,88 & 0,18 & 0,85 \\
\hline Stone.weight_2019 & 0,96 & 0,01 & 0,13 & 0,23 & 0,84 & 0,46 & 0,02 & 0,07 & 0,35 & 0,07 \\
\hline Height_2019 & 0,81 & 0,05 & 0,11 & 0,56 & 0,04 & 0,25 & 0,49 & 0,60 & 0,11 & 0,87 \\
\hline LateralWidth_2019 & 0,62 & 0,01 & 0,16 & 0,62 & 0,13 & 0,10 & 0,18 & 0,90 & 0,16 & 0,74 \\
\hline VentralWidth_2019 & 0,89 & 0,01 & 0,23 & 0,50 & 0,32 & 0,09 & 0,04 & 0,45 & 0,21 & 0,41 \\
\hline Firmness_2019 & 0,97 & 0,62 & 0,28 & 0,72 & 0,75 & 0,22 & 0,44 & 0,87 & 0,26 & 0,46 \\
\hline
\end{tabular}


Table S1. (Cont.)

\begin{tabular}{|c|c|c|c|c|c|c|c|c|c|c|}
\hline & & & & & & & & & & \\
\hline Citric_F_2016 & $-0,62$ & 0,17 & 0,32 & $-0,02$ & 0,36 & 0,16 & $-0,53$ & 0,43 & $-0,06$ & 0,18 \\
\hline Malic_F_2016 & $-0,39$ & 0,11 & $-0,45$ & 0,34 & 0,06 & $-0,38$ & 0,08 & 0,06 & $-0,44$ & 0,22 \\
\hline Succinic_F_2016 & $-0,25$ & $-0,53$ & 0,07 & $-0,62$ & $-0,33$ & $-0,40$ & $-0,26$ & $-0,34$ & 0,30 & $-0,48$ \\
\hline Fumaric_F2016 & $-0,44$ & $-0,60$ & $-0,03$ & $-0,53$ & $-0,46$ & $-0,27$ & $-0,18$ & $-0,32$ & 0,21 & $-0,42$ \\
\hline Citric_P_2016 & $-0,28$ & 0,81 & 0,14 & 0,76 & 0,74 & 0,42 & $-0,11$ & 0,17 & $-0,44$ & 0,23 \\
\hline Malic_P_2016 & $-0,26$ & $-0,48$ & $-0,28$ & $-0,31$ & $-0,46$ & $-0,37$ & $-0,45$ & 0,36 & 0,16 & 0,21 \\
\hline Succinic_P_2016 & 0,41 & $-0,69$ & 0,08 & $-0,81$ & $-0,56$ & $-0,34$ & $-0,01$ & $-0,21$ & 0,56 & $-0,36$ \\
\hline Fumaric_P_2016 & $-0,26$ & $-0,01$ & $-0,57$ & 0,24 & $-0,02$ & $-0,45$ & 0,49 & $-0,09$ & $-0,35$ & 0,04 \\
\hline Citric_P_2017 & $-0,26$ & 0,74 & 0,17 & 0,71 & 0,64 & 0,35 & $-0,16$ & 0,06 & $-0,40$ & 0,14 \\
\hline Malic_P_2017 & $-0,37$ & $-0,69$ & $-0,26$ & $-0,56$ & $-0,66$ & $-0,42$ & $-0,36$ & 0,32 & 0,37 & 0,23 \\
\hline Succinic_P_2017 & 0,23 & $-0,81$ & 0,05 & $-0,90$ & $-0,63$ & $-0,42$ & $-0,32$ & $-0,33$ & 0,56 & $-0,53$ \\
\hline Fumaric_P_2017 & $-0,08$ & 0,00 & 0,10 & $-0,13$ & $-0,05$ & 0,28 & $-0,28$ & $-0,07$ & 0,05 & 0,25 \\
\hline Citric_P_2019 & $-0,50$ & 0,65 & 0,19 & 0,63 & 0,56 & 0,27 & $-0,19$ & 0,22 & $-0,39$ & 0,30 \\
\hline Malic_P_2019 & $-0,72$ & $-0,42$ & $-0,22$ & $-0,29$ & $-0,30$ & $-0,11$ & $-0,37$ & 0,48 & $-0,06$ & 0,43 \\
\hline Succinic_P_2019 & 0,00 & $-0,46$ & 0,36 & $-0,72$ & $-0,27$ & 0,03 & $-0,71$ & $-0,06$ & 0,47 & $-0,21$ \\
\hline Fumaric_P_2019 & $-0,20$ & 0,04 & 0,37 & $-0,25$ & 0,24 & 0,52 & $-0,35$ & 0,25 & 0,09 & 0,16 \\
\hline Ascorbic_F_2016 & 0,17 & $-0,39$ & $-0,32$ & $-0,46$ & $-0,28$ & $-0,14$ & 0,20 & $-0,64$ & 0,15 & $-0,34$ \\
\hline Ascorbic_P_2016 & 0,04 & $-0,28$ & $-0,07$ & $-0,19$ & $-0,50$ & $-0,01$ & $-0,22$ & 0,24 & 0,36 & 0,39 \\
\hline Ascorbic_P_2017 & 0,02 & $-0,57$ & 0,22 & $-0,71$ & $-0,58$ & $-0,11$ & $-0,19$ & 0,10 & 0,63 & 0,09 \\
\hline Ascorbic_P_2019 & 0,71 & 0,32 & 0,23 & 0,21 & 0,24 & 0,28 & 0,15 & $-0,47$ & 0,01 & $-0,40$ \\
\hline Fructose_F_2016 & $-0,27$ & $-0,64$ & $-0,06$ & $-0,56$ & $-0,60$ & $-0,21$ & 0,16 & $-0,04$ & 0,26 & 0,03 \\
\hline Glucose_F_2016 & $-0,17$ & $-0,52$ & $-0,26$ & $-0,47$ & $-0,45$ & $-0,27$ & 0,11 & 0,01 & 0,18 & 0,11 \\
\hline Sucrose_F_2016 & 0,07 & $-0,29$ & $-0,15$ & $-0,28$ & $-0,15$ & $-0,57$ & 0,01 & 0,16 & 0,11 & $-0,13$ \\
\hline Fructose_P_2016 & $-0,46$ & $-0,57$ & $-0,42$ & $-0,29$ & $-0,62$ & $-0,36$ & 0,16 & $-0,06$ & $-0,02$ & 0,17 \\
\hline Glucose_P_2016 & $-0,12$ & $-0,64$ & $-0,32$ & $-0,49$ & $-0,68$ & $-0,33$ & 0,30 & $-0,06$ & 0,23 & 0,17 \\
\hline Sucrose_P_2016 & 0,56 & $-0,29$ & 0,14 & $-0,41$ & $-0,15$ & $-0,21$ & 0,13 & $-0,41$ & 0,34 & $-0,69$ \\
\hline Fructose_P_2017 & $-0,32$ & $-0,01$ & $-0,02$ & $-0,06$ & 0,11 & 0,22 & 0,12 & $-0,38$ & $-0,02$ & $-0,31$ \\
\hline Glucose_P_2017 & 0,24 & $-0,16$ & $-0,06$ & $-0,03$ & $-0,14$ & 0,06 & 0,66 & $-0,64$ & $-0,19$ & $-0,52$ \\
\hline Sucrose_P_2017 & 0,59 & $-0,16$ & 0,35 & $-0,28$ & $-0,20$ & 0,13 & 0,20 & $-0,56$ & 0,32 & $-0,55$ \\
\hline Fructose_P_2019 & $-0,52$ & $-0,25$ & $-0,47$ & $-0,01$ & $-0,26$ & 0,01 & 0,28 & 0,09 & $-0,30$ & 0,42 \\
\hline Glucose_P_2019 & $-0,12$ & $-0,78$ & $-0,34$ & $-0,56$ & $-0,83$ & $-0,39$ & 0,33 & 0,03 & 0,28 & 0,14 \\
\hline Sucrose_P_2019 & 1 & $-0,05$ & $-0,04$ & $-0,17$ & $-0,09$ & $-0,21$ & 0,25 & $-0,47$ & 0,36 & $-0,48$ \\
\hline Weight_2016 & 0,57 & 1 & 0,36 & 0,86 & 0,91 & 0,68 & $-0,02$ & 0,29 & $-0,46$ & 0,35 \\
\hline Stone.weight_2016 & 0,81 & 0,48 & 1 & $-0,04$ & 0,29 & 0,62 & $-0,53$ & 0,18 & 0,40 & 0,03 \\
\hline Height_2016 & 0,55 & $3,94 \mathrm{E}+0$ & 0,999 & 1 & 0,70 & 0,43 & 0,30 & 0,34 & $-0,71$ & 0,46 \\
\hline LateralWidth_2016 & 0,50 & $5,07 E+0$ & 0,64 & 0,00 & 1 & 0,67 & $-0,09$ & 0,14 & $-0,56$ & 0,14 \\
\hline VentralWidth_2016 & 0,44 & 0,00 & 0,17 & 0,03 & 0,00 & 1 & $-0,18$ & 0,20 & $-0,23$ & 0,29 \\
\hline Firmness_2016 & 0,30 & 0,88 & 0,13 & 0,53 & 0,49 & 0,39 & 1 & $-0,26$ & $-0,39$ & $-0,07$ \\
\hline Weight_2017 & 0,30 & 0,15 & 0,68 & 0,23 & 0,13 & 0,17 & 0,31 & 1 & $-0,05$ & 0,88 \\
\hline Stone.weight_2017 & 0,76 & 0,03 & 0,27 & 0,00 & 0,02 & 0,19 & 0,27 & 0,88 & 1 & $-0,22$ \\
\hline Height_2017 & 0,07 & 0,12 & 0,54 & 0,05 & 0,24 & 0,32 & 0,95 & 0,00 & 0,37 & 1 \\
\hline LateralWidth_2017 & 0,06 & 0,41 & 0,54 & 0,60 & 0,29 & 0,27 & 0,20 & 0,00 & 0,88 & 0,02 \\
\hline VentralWidth_2017 & 0,25 & 0,45 & 0,43 & 0,71 & 0,36 & 0,16 & 0,35 & 0,00 & 0,71 & 0,05 \\
\hline Firmness_2017 & 0,31 & 0,54 & 0,95 & 0,70 & 0,35 & 0,41 & 0,34 & 0,73 & 0,60 & 0,84 \\
\hline Weight_2019 & 0,38 & 0,07 & 0,49 & 0,01 & 0,34 & 0,22 & 0,13 & 0,52 & 0,11 & 0,02 \\
\hline Stone.weight_2019 & 0,67 & 0,38 & 0,66 & 0,55 & 0,20 & 0,72 & 0,41 & 0,27 & 0,66 & 0,82 \\
\hline Height_2019 & 0,37 & 0,14 & 0,20 & 0,02 & 0,45 & 0,46 & 0,11 & 0,63 & 0,09 & 0,02 \\
\hline LateralWidth_2019 & 0,34 & 0,13 & 0,49 & 0,03 & 0,46 & 0,29 & 0,17 & 0,88 & 0,15 & 0,08 \\
\hline VentralWidth_2019 & 0,51 & 0,19 & 0,83 & 0,09 & 0,56 & 0,17 & 0,17 & 0,95 & 0,28 & 0,19 \\
\hline rmne & 0,07 & 0,59 & 0,71 & 0,62 & 0,82 & 0,78 & 0,63 & 0,26 & 0,85 & 0,62 \\
\hline
\end{tabular}


Table S1. (Cont.)

\begin{tabular}{|c|c|c|c|c|c|c|c|c|c|}
\hline & $\begin{array}{l}\text { Lateral } \\
\text { Width }\end{array}$ & $\begin{array}{l}\text { Ventral } \\
\text { Width }\end{array}$ & $\begin{array}{c}\text { Firmness } \\
2017 \\
\end{array}$ & $\begin{array}{c}\text { Weight } \\
2019\end{array}$ & $\begin{array}{l}\text { Stone. } \\
\text { weight }\end{array}$ & $\begin{array}{c}\text { Height } \\
2019 \\
\end{array}$ & $\begin{array}{c}\text { LateralWidth_ } \\
2019\end{array}$ & $\begin{array}{l}\text { Ventral } \\
\text { Width }\end{array}$ & $\begin{array}{c}\text { Firmness } \\
2019\end{array}$ \\
\hline Citric_F_2016 & 0,50 & 0,26 & $-0,66$ & $-0,37$ & $-0,80$ & $-0,41$ & $-0,36$ & $-0,46$ & $-0,13$ \\
\hline Malic_F_2016 & $-0,03$ & $-0,36$ & $-0,15$ & 0,12 & $-0,06$ & 0,15 & 0,14 & $-0,10$ & 0,25 \\
\hline Succinic_F_2016 & $-0,13$ & $-0,35$ & $-0,49$ & $-0,56$ & $-0,33$ & $-0,57$ & $-0,60$ & $-0,55$ & 0,40 \\
\hline Fumaric_F2016 & $-0,17$ & $-0,16$ & 0,06 & $-0,29$ & 0,14 & $-0,24$ & $-0,18$ & $-0,19$ & 0,75 \\
\hline Citric_P_2016 & $-0,16$ & $-0,19$ & $-0,36$ & 0,40 & $-0,12$ & 0,41 & 0,40 & 0,24 & $-0,24$ \\
\hline Malic_P_2016 & 0,32 & 0,22 & $-0,05$ & $-0,32$ & 0,00 & $-0,21$ & $-0,25$ & $-0,26$ & $-0,03$ \\
\hline Succinic_P_2016 & 0,13 & 0,12 & 0,13 & $-0,58$ & $-0,14$ & $-0,62$ & $-0,64$ & $-0,43$ & 0,03 \\
\hline Fumaric_P_2016 & $-0,16$ & $-0,41$ & 0,11 & 0,11 & $-0,04$ & 0,13 & 0,15 & $-0,10$ & 0,30 \\
\hline Citric_P_2017 & $-0,28$ & $-0,29$ & $-0,33$ & 0,40 & 0,00 & 0,42 & 0,39 & 0,26 & $-0,10$ \\
\hline Malic_P_2017 & 0,38 & 0,27 & $-0,22$ & $-0,30$ & 0,03 & $-0,22$ & $-0,27$ & $-0,26$ & 0,22 \\
\hline Succinic_P_2017 & $-0,04$ & $-0,09$ & $-0,11$ & $-0,70$ & $-0,10$ & $-0,69$ & $-0,73$ & $-0,52$ & 0,16 \\
\hline Fumaric_P_2017 & 0,26 & 0,30 & 0,26 & 0,18 & 0,45 & 0,18 & 0,27 & 0,21 & 0,53 \\
\hline Citric_P_2019 & $-0,01$ & $-0,11$ & $-0,31$ & 0,34 & $-0,12$ & 0,33 & 0,35 & 0,16 & 0,08 \\
\hline Malic_P_2019 & 0,68 & 0,52 & $-0,24$ & $-0,17$ & $-0,22$ & $-0,12$ & $-0,07$ & $-0,16$ & 0,26 \\
\hline Succinic_P_2019 & 0,27 & 0,23 & $-0,42$ & $-0,57$ & $-0,23$ & $-0,58$ & $-0,60$ & $-0,41$ & 0,05 \\
\hline Fumaric_P_2019 & 0,53 & 0,54 & $-0,59$ & $-0,16$ & $-0,50$ & $-0,22$ & $-0,20$ & $-0,07$ & $-0,26$ \\
\hline Ascorbic_F_2016 & $-0,23$ & $-0,27$ & 0,25 & $-0,05$ & 0,43 & $-0,04$ & 0,02 & $-0,03$ & 0,66 \\
\hline Ascorbic_P_2016 & 0,18 & 0,38 & 0,31 & 0,25 & 0,69 & 0,32 & 0,28 & 0,34 & 0,21 \\
\hline Ascorbic_P_2017 & 0,42 & 0,51 & 0,28 & $-0,27$ & 0,18 & $-0,30$ & $-0,26$ & $-0,16$ & 0,41 \\
\hline Ascorbic_P_2019 & $-0,62$ & $-0,38$ & 0,02 & 0,23 & 0,30 & 0,21 & 0,12 & 0,32 & $-0,37$ \\
\hline Fructose_F_2016 & 0,20 & 0,19 & $-0,36$ & 0,00 & 0,01 & $-0,03$ & $-0,10$ & 0,06 & 0,39 \\
\hline Glucose_F_2016 & 0,24 & 0,10 & $-0,47$ & $-0,08$ & $-0,11$ & $-0,10$ & $-0,18$ & $-0,10$ & 0,17 \\
\hline Sucrose_F_2016 & 0,22 & $-0,12$ & $-0,25$ & $-0,66$ & $-0,74$ & $-0,69$ & $-0,73$ & $-0,73$ & $-0,36$ \\
\hline Fructose_P_2016 & 0,07 & 0,00 & $-0,16$ & 0,23 & 0,35 & 0,27 & 0,22 & 0,21 & 0,65 \\
\hline Glucose_P_2016 & 0,21 & 0,18 & 0,04 & 0,12 & 0,32 & 0,11 & 0,07 & 0,13 & 0,53 \\
\hline Sucrose_P_2016 & $-0,33$ & $-0,25$ & 0,36 & $-0,54$ & $-0,16$ & $-0,56$ & $-0,53$ & $-0,39$ & $-0,16$ \\
\hline Fructose_P_2017 & $-0,35$ & $-0,26$ & $-0,22$ & 0,17 & 0,16 & 0,19 & 0,23 & 0,17 & 0,46 \\
\hline Glucose_P_2017 & $-0,52$ & $-0,29$ & 0,37 & 0,29 & 0,34 & 0,25 & 0,28 & 0,41 & 0,34 \\
\hline Sucrose_P_2017 & $-0,46$ & $-0,15$ & 0,41 & 0,02 & 0,41 & $-0,03$ & $-0,03$ & 0,21 & 0,15 \\
\hline Fructose_P_2019 & 0,30 & 0,26 & $-0,02$ & 0,46 & 0,32 & 0,48 & 0,52 & 0,42 & 0,55 \\
\hline Glucose_P_2019 & 0,24 & 0,30 & 0,26 & 0,05 & 0,35 & 0,06 & 0,05 & 0,13 & 0,48 \\
\hline Sucrose_P_2019 & $-0,52$ & $-0,44$ & 0,32 & $-0,22$ & 0,21 & $-0,23$ & $-0,31$ & $-0,16$ & $-0,39$ \\
\hline Weight_2016 & 0,05 & 0,12 & $-0,16$ & 0,46 & $-0,20$ & 0,42 & 0,45 & 0,35 & $-0,48$ \\
\hline Stone.weight_2016 & 0,12 & 0,36 & $-0,40$ & 0,02 & $-0,16$ & $-0,06$ & $-0,09$ & 0,11 & $-0,16$ \\
\hline Height_2016 & $-0,01$ & 0,05 & 0,04 & 0,66 & $-0,04$ & 0,65 & 0,67 & 0,52 & $-0,38$ \\
\hline LateralWidth_2016 & 0,09 & 0,05 & $-0,24$ & 0,20 & $-0,46$ & 0,14 & 0,23 & 0,10 & $-0,45$ \\
\hline VentralWidth_2016 & 0,22 & 0,49 & $-0,26$ & 0,50 & $-0,04$ & 0,43 & 0,50 & 0,55 & $-0,16$ \\
\hline Firmness_2016 & $-0,33$ & $-0,25$ & 0,45 & 0,46 & 0,26 & 0,44 & 0,45 & 0,42 & 0,03 \\
\hline Weight_2017 & 0,78 & 0,76 & $-0,17$ & 0,18 & $-0,30$ & 0,18 & 0,17 & 0,10 & $-0,39$ \\
\hline Stone.weight_2017 & $-0,10$ & 0,05 & $-0,06$ & $-0,41$ & 0,23 & $-0,39$ & $-0,50$ & $-0,30$ & 0,06 \\
\hline Height_2017 & 0,74 & 0,76 & $-0,06$ & 0,49 & $-0,02$ & 0,49 & 0,49 & 0,38 & $-0,17$ \\
\hline LateralWidth_2017 & 1 & 0,87 & $-0,14$ & $-0,06$ & $-0,44$ & $-0,10$ & $-0,02$ & $-0,10$ & $-0,11$ \\
\hline VentralWidth_2017 & 0,00 & 1 & $-0,05$ & 0,24 & $-0,13$ & 0,20 & 0,26 & 0,28 & $-0,08$ \\
\hline Firmness_2017 & 0,82 & 0,87 & 1 & 0,08 & 0,49 & 0,12 & 0,23 & 0,11 & 0,26 \\
\hline Weight_2019 & 0,88 & 0,72 & 0,84 & 1 & 0,52 & 0,99 & 0,96 & 0,96 & 0,08 \\
\hline Stone.weight_2019 & 0,26 & 0,64 & 0,23 & 0,26 & 1 & 0,58 & 0,54 & 0,59 & 0,48 \\
\hline Height_2019 & 0,96 & 0,99 & 0,83 & $5,19 E+07$ & 0,28 & 1 & 0,97 & 0,95 & 0,11 \\
\hline LateralWidth_2019 & 0,85 & 0,97 & 0,59 & $1,70 \mathrm{E}+08$ & 0,13 & $8,73 E+0$ & 1 & 0,93 & 0,19 \\
\hline VentralWidth_2019 & 0,86 & 0,74 & 0,80 & $9,15 E+09$ & 0,05 & 0,00 & $5,02 E+09$ & 1 & 0,13 \\
\hline Firmness_2019 & 0,97 & 0,54 & 0,63 & 0,81 & 0,83 & 0,92 & 0,72 & 0,99 & 1 \\
\hline
\end{tabular}


Table S2. Sweetness estimation, SI and TSI were calculated according to Magwaza and Opara (2015).

\begin{tabular}{l|cccc|cccc}
\cline { 2 - 9 } \multicolumn{1}{c}{ Genotype } & \multicolumn{9}{c}{ SI } & \multicolumn{3}{c}{ TSI } \\
\cline { 2 - 9 } & Flesh & Peel & Peel & Peel & Flesh & Peel & Peel & Peel \\
\hline 'Goldrich' & 9,33 & $\mathbf{2 0 1 6}$ & $\mathbf{2 0 1 7}$ & $\mathbf{2 0 1 9}$ & $\mathbf{2 0 1 6}$ & $\mathbf{2 0 1 6}$ & $\mathbf{2 0 1 7}$ & $\mathbf{2 0 1 9}$ \\
'Canino' & 10,43 & 63,67 & 30,74 & 58,22 & 6,89 & 37,20 & 21,68 & 42,14 \\
'Mitger' & 8,50 & 66,22 & 81,45 & 80,63 & 7,72 & 47,00 & 46,34 & 59,55 \\
'Tadeo' & 13,88 & 70,66 & 63,02 & 82,27 & 6,25 & 48,22 & 59,60 & 57,32 \\
'Dama Rosa' & 14,40 & 55,04 & 23,08 & 67,85 & 10,24 & 51,79 & 45,63 & 60,56 \\
'Dama & 12,04 & 52,27 & 44,83 & 53,57 & 8,66 & 40,15 & 16,66 & 49,50 \\
Taronja' & & & & 38,13 & 32,17 & 38,89 \\
'GG9310' & 10,56 & 54,46 & 46,20 & 52,07 & 7,81 & 39,70 & 33,27 & 38,16 \\
'GG979' & 7,56 & 50,54 & 44,51 & 64,04 & 5,60 & 36,91 & 31,82 & 46,65 \\
'GP9817' & 9,98 & 59,19 & 52,29 & 53,85 & 7,38 & 43,24 & 37,61 & 39,19 \\
'HG9821' & 10,74 & 62,78 & 92,30 & 70,66 & 7,93 & 45,73 & 67,37 & 51,25 \\
'HG9850' & 7,55 & 61,78 & 66,43 & 69,67 & 5,58 & 45,34 & 48,50 & 51,04 \\
'HM964' & 8,82 & 54,53 & 76,46 & 52,73 & 6,51 & 39,79 & 56,03 & 38,70 \\
'SEOP934' & 16,30 & 63,58 & 43,30 & 60,28 & 12,05 & 46,60 & 31,20 & 44,22 \\
\hline
\end{tabular}

Table S3. Used primers in the qPCR amplification for sugar-related and reference genes

\begin{tabular}{|c|c|c|}
\hline Gene & Forward $\left(5^{\prime}-3^{\prime}\right)$ & Reverse $\left(5^{\prime}-3^{\prime}\right)$ \\
\hline ParFK1 & GAGTTCTTGACTGGAGGTGATG & CTGCTGCCCCTACAGTTACTATCAGA \\
\hline ParSDH & GAAGCCAGCAGAGATGGTTG & ACATATGCCAACAGCCTTGAGT \\
\hline Parsus1 & CCCTTCAGAACAGAGAAGGGTAT & TCAGGGTACTTTGTCTTCTCCAG \\
\hline ParsUS3 & AACTGAGGGATCTGGTAAACCTC & AGCACCTCGTGTATCTGCTATGT \\
\hline Parsus6 & CTCAGGACTAGTTGAATGGTTCG & ATCGATCAGTCTGAGCTGCTATC \\
\hline ParSPS1 & AGACGTGGATTAGTCAGCAGAAG & CACCATAGCTCCAATCTACATCC \\
\hline ParSPS2 & CTGAAGGAGAGAAAGGAGACACA & GTATCAGAATCACGACCAAGCTC \\
\hline ParSPS3 & GATGGTGCTCTAGCTCACATTCT & GTTTCTTCCAAGTGAGTGTCCTG \\
\hline Actin & СTTCTTACTGAGGCACCCCTGAAT & AGCATAGAGGGAGAGAACTGCTTG \\
\hline SAND & TCGTGGGTACCAGGAAAACGACAT & CCTGCTAGCTTGTGTTCATCTCCA \\
\hline
\end{tabular}


Table S4. Sugar content (g/100gDW) in the studied accessions in 2019 and 2020.

\begin{tabular}{|c|c|c|c|c|c|c|c|c|c|c|c|c|}
\hline \multirow{3}{*}{ Genotype } & \multicolumn{9}{|c|}{2019} & & & \\
\hline & \multicolumn{3}{|c|}{ Sucrose } & \multicolumn{3}{|c|}{ Glucose } & \multicolumn{3}{|c|}{ Fructose } & \multicolumn{3}{|c|}{ Sorbitol } \\
\hline & Mean & sd & sig & Mean & $\mathrm{sd}$ & sig & Mean & sd & sig & Mean & sd & sig \\
\hline 'Canino' & 50.45 & 0.19 & $\mathrm{~g}$ & 5.19 & 0.1 & $\mathrm{a}$ & 1.87 & 0.0 & $a$ & 8.29 & 0.6 & de \\
\hline 'Dama Rosa' & 29.81 & 0.36 & de & 13.35 & 0.9 & $f$ & 5.01 & 0.2 & $d$ & 5.05 & 1.7 & $a b c d$ \\
\hline $\begin{array}{c}\text { 'Dama } \\
\text { Taronja' }\end{array}$ & 17.14 & 3.62 & $a$ & 12.51 & $\begin{array}{c}0.2 \\
2\end{array}$ & $f$ & 5.12 & $\begin{array}{c}0.1 \\
7\end{array}$ & $d$ & 5.60 & $\begin{array}{c}0.9 \\
8\end{array}$ & bcd \\
\hline 'GG9310' & 27.44 & 0.69 & $\mathrm{~cd}$ & 8.28 & 0.1 & c & 3.09 & 0.0 & $b$ & 3.38 & 0.8 & $a b c$ \\
\hline 'GG979' & 36.65 & 0.05 & $f$ & 10.76 & 0.3 & $\mathrm{e}$ & 5.29 & 0.0 & $d$ & 2.34 & 0.7 & $a b$ \\
\hline 'Goldrich' & 32.74 & 0.09 & de & 17.13 & 0.0 & g & 5.58 & 0.0 & de & 2.12 & 0.8 & $a b c$ \\
\hline 'GP9817' & 19.53 & 0.15 & $a b$ & 12.89 & 0.0 & $f$ & 5.15 & 1.0 & $d$ & 3.86 & 0.0 & $a b c$ \\
\hline 'HG9821' & 33.89 & 1.29 & ef & 10.79 & 0.4 & e & 6.52 & 0.2 & $f$ & 2.12 & 0.6 & $\mathrm{a}$ \\
\hline 'HG9850' & 32.63 & 0.68 & ef & 7.50 & 0.1 & $\mathrm{bc}$ & 4.22 & 0.0 & c & 8.18 & 1.2 & de \\
\hline 'HM964' & 24.58 & 0.36 & $\mathrm{bc}$ & 6.75 & 0.2 & $b$ & 3.01 & 0.1 & $b$ & 6.10 & 1.9 & $\mathrm{~cd}$ \\
\hline 'Mitger' & 27.64 & 0.03 & $\mathrm{~cd}$ & 16.63 & 0.1 & $\mathrm{~g}$ & 5.97 & 0.1 & ef & 13.15 & 1.3 & $f$ \\
\hline 'SEOP934' & 25.40 & 3.21 & c & 9.49 & 0.3 & d & 3.51 & 0.2 & $\mathrm{~b}$ & 9.43 & 1.4 & $\mathrm{e}$ \\
\hline \multicolumn{13}{|c|}{2020} \\
\hline \multirow{2}{*}{ Genotype } & \multicolumn{3}{|c|}{ Sucrose } & \multicolumn{3}{|c|}{ Glucose } & \multicolumn{3}{|c|}{ Fructose } & \multicolumn{3}{|c|}{ Sorbitol } \\
\hline & Mean & sd & sig & Mean & $\mathrm{sd}$ & sig & Mean & sd & sig & Mean & $\mathrm{sd}$ & sig \\
\hline 'Canino' & 39.33 & 0.57 & $f$ & 6.68 & 0.1 & a & 2.41 & 0.0 & $a$ & 3.23 & 0.0 & $a b c d$ \\
\hline 'Dama Rosa' & 25.73 & 0.82 & d & 9.00 & 0.3 & $\mathrm{bc}$ & 4.19 & 0.1 & $b$ & 3.07 & 0.1 & $a b c d$ \\
\hline $\begin{array}{c}\text { 'Dama } \\
\text { Taronja' }\end{array}$ & 22.33 & 0.16 & c & 16.97 & $\begin{array}{c}0.2 \\
5\end{array}$ & e & 7.29 & $\begin{array}{c}0.0 \\
3\end{array}$ & e & 5.26 & $\begin{array}{c}1.2 \\
3\end{array}$ & de \\
\hline 'GG9310' & 28.17 & 2.21 & d & 10.91 & 0.1 & c & 4.53 & 0.7 & $b$ & 2.65 & 0.7 & $a b c d$ \\
\hline 'GG979' & 17.04 & 1.98 & $a b$ & 16.72 & 2.3 & $\mathrm{e}$ & 5.49 & 0.7 & $\mathrm{~cd}$ & 2.60 & 0.3 & $a b c$ \\
\hline Goldrich & 15.98 & 0.18 & $\mathrm{a}$ & 14.44 & 0.0 & $d$ & 5.76 & 0.0 & $d$ & 2.10 & 0.5 & $\mathrm{a}$ \\
\hline 'GP9817' & 21.95 & 0.05 & c & 14.04 & 0.3 & $d$ & 4.66 & 0.1 & $\mathrm{bc}$ & 4.96 & 1.4 & bcde \\
\hline 'HG9821' & 32.70 & 0.53 & e & 10.10 & 0.5 & $\mathrm{bc}$ & 5.65 & 0.2 & $d$ & 2.39 & 0.4 & $a b$ \\
\hline 'HG9850' & 32.57 & 0.98 & e & 9.04 & 0.3 & $\mathrm{bc}$ & 4.66 & 0.1 & $\mathrm{bc}$ & 10.35 & 1.6 & $f$ \\
\hline 'HM964' & 31.27 & 0.99 & e & 8.36 & 0.5 & $a b$ & 4.56 & 0.1 & $b$ & 5.94 & 1.5 & $\mathrm{e}$ \\
\hline 'Mitger' & 19.15 & 0.01 & $b$ & 17.85 & 0.2 & e & 6.83 & 0.0 & e & 12.56 & 1.5 & $f$ \\
\hline 'SEOP934' & 26.03 & 0.06 & d & 14.04 & 0.0 & $d$ & 2.98 & 0.0 & $a$ & 5.19 & 0.4 & cde \\
\hline
\end{tabular}


Table S5. Homologous proteins in other species which sugar content in fruit has economically importance in Prunus persica (A) and Prunus armeniaca (B). Ev: E-Value

\begin{tabular}{|c|c|c|c|c|c|c|c|c|c|c|c|}
\hline \multirow{2}{*}{ Gene } & \multicolumn{3}{|c|}{ Prunus persica } & \multicolumn{4}{|c|}{ Vitis vinifera } & \multicolumn{4}{|c|}{ Fragaria vesca } \\
\hline & Sequence ID & Gene location & LG & Obtained sequence & Position & Identity & Ev & Obtained sequence & Position & Identity & Ev \\
\hline PperSDH & Prupe.8G143000.1 & $\begin{array}{c}\text { Pp08: } \\
\text { 15999040- } \\
\text { 16001622+ }\end{array}$ & 8 & GSVIVT01010642001 & $\begin{array}{c}\text { chr16: } \\
\text { 15675424-15679159 } \\
\text { fwd }\end{array}$ & $\begin{array}{l}280 / 349 \\
(80.23 \%)\end{array}$ & 0 & $\begin{array}{c}\text { mrna13340.1-v1.0- } \\
\text { hybrid }\end{array}$ & $\begin{array}{c}\text { LG7: } \\
\text { 22062128-22064232 } \\
\text { rev }\end{array}$ & $\begin{array}{l}289 / 355 \\
(81.41 \%)\end{array}$ & 0 \\
\hline PperFK1 & Prupe.2G151100.1 & $\begin{array}{c}\text { Pp02: } \\
\text { 20597751- } \\
20600680+\end{array}$ & 2 & GSVIVT01027578001 & $\begin{array}{c}\text { chr15: } \\
\text { 15399528-15404025 } \\
\text { rev }\end{array}$ & $\begin{array}{l}275 / 367 \\
(74.93 \%)\end{array}$ & $\begin{array}{c}2.76 \cdot 10^{-} \\
166\end{array}$ & $\begin{array}{l}\text { mrna19425.1-v1.0- } \\
\text { hybrid }\end{array}$ & $\begin{array}{c}\text { LG7: } \\
\text { 4380104-4385219 } \\
\text { fwd }\end{array}$ & $\begin{array}{c}62 / 73 \\
(84.9 \%)\end{array}$ & $7.5 \cdot 10^{-77}$ \\
\hline PperSUS1 & Prupe.7G192300.1 & $\begin{array}{c}\text { Pp07: } \\
\text { 18350336- } \\
\text { 18356360+ }\end{array}$ & 7 & GSVIVT01015018001 & $\begin{array}{c}\text { chr11: } \\
490468-494415 \text { fwd }\end{array}$ & $\begin{array}{l}670 / 806 \\
(83.13 \%)\end{array}$ & 0 & mrna12940.1-v1.0- & $\begin{array}{c}\text { LG1: } \\
6534488-6537776\end{array}$ & $\begin{array}{r}719 / 806 \\
(89.21 \%)\end{array}$ & 0 \\
\hline PperSUS3 & Prupe.8G264300.1 & $\begin{array}{c}\text { Pp08: } \\
22179197- \\
22184773\end{array}$ & 8 & chr12 & $\begin{array}{c}\text { chr12: } \\
\text { 8810872-8811602+ }\end{array}$ & $\begin{array}{c}47 / 57 \\
(82.46 \%)\end{array}$ & $1.58 \cdot 10^{-19}$ & hybrid & $\begin{array}{c}6534488-653 / 1 / 6 \\
\text { rev }\end{array}$ & $\begin{array}{l}574 / 810 \\
(70.86 \%)\end{array}$ & 0 \\
\hline PperSUS6 & Prupe.5G241700.1 & $\begin{array}{c}\text { Pp05: } \\
\text { 18195911- } \\
\text { 18200676+ }\end{array}$ & 5 & GSVIVT01029388001 & $\begin{array}{c}\text { chr17: } \\
\text { 15994779-15999389 } \\
\text { fwd }\end{array}$ & $\begin{array}{l}660 / 901 \\
(73.25 \%)\end{array}$ & 0 & $\begin{array}{l}\text { mrna09290.1-v1.0- } \\
\text { hybrid }\end{array}$ & $\begin{array}{c}\text { LG5: } \\
\text { 9513235-9523248 } \\
\text { rev }\end{array}$ & $\begin{array}{l}747 / 912 \\
(81.91 \%)\end{array}$ & 0 \\
\hline PperSPS1 & Prupe.7G249900.1 & $\begin{array}{c}\text { Pp07: } \\
\text { 21151882- } \\
\text { 21157785- }\end{array}$ & 7 & GSVIVT01012825001 & $\begin{array}{c}\text { chr11: } \\
\text { 5766519-5776338 } \\
\text { reverse }\end{array}$ & $\begin{array}{l}878 / 1057 \\
(83.07 \%),\end{array}$ & 0 & $\begin{array}{c}\text { mrna31122.1-v1.0- } \\
\text { hybrid }\end{array}$ & $\begin{array}{c}\text { LG1: } \\
\text { 2640599-2646376 } \\
\text { rev }\end{array}$ & $\begin{array}{l}956 / 1057 \\
(90.44 \%)\end{array}$ & 0 \\
\hline PperSPS2 & Prupe.1G483200.1 & $\begin{array}{c}\text { Pp01: } \\
\text { 40288494- } \\
\text { 40295210- }\end{array}$ & 1 & GSVIVT01035882001 & $\begin{array}{c}\text { chr4: } \\
5217811 . .5232786 \mathrm{rev}\end{array}$ & $\begin{array}{l}816 / 1059 \\
(77.05 \%)\end{array}$ & 0 & $\begin{array}{l}\text { mrna11606.1-v1.0- } \\
\text { hybrid }\end{array}$ & $\begin{array}{c}\text { unanchored: } \\
\text { 9174890-9180436 } \\
\text { fwd }\end{array}$ & $\begin{array}{l}745 / 895 \\
(83.24 \%)\end{array}$ & 0 \\
\hline PperSPS3 & Prupe.1G159700.1 & $\begin{array}{c}\text { Pp01: } \\
\text { 12702147- } \\
\text { 12709381- }\end{array}$ & 1 & GSVIVT01020928001 & $\begin{array}{c}\text { chr5: } \\
\text { 16983726-16990038 } \\
\text { rev }\end{array}$ & $\begin{array}{l}878 / 1067 \\
(82.29 \%)\end{array}$ & 0 & $\begin{array}{c}\text { mrna06523.1-v1.0- } \\
\text { hybrid }\end{array}$ & $\begin{array}{c}\text { LG4: } \\
\text { 16876681-16882157 } \\
\text { rev }\end{array}$ & $\begin{array}{c}973 / 1068 \\
(91.1 \%)\end{array}$ & 0 \\
\hline
\end{tabular}


Table S5. A. (Cont.)

\begin{tabular}{|c|c|c|c|c|c|c|c|c|c|c|c|}
\hline \multirow{2}{*}{ Gene } & \multicolumn{3}{|c|}{ Prunus persica } & \multicolumn{4}{|c|}{ Malus domestica } & \multicolumn{4}{|c|}{ Prunus dulcis } \\
\hline & Sequence ID & Gene location & LG & Obtained sequence & Position & Identity & Ev & Obtained sequence & Position & Identity & Ev \\
\hline PperSDH & Prupe.8G143000.1 & $\begin{array}{l}\text { Pp08: } \\
\text { 15999040- } \\
\text { 6001622+ }\end{array}$ & 8 & MDP0000188052 & $\begin{array}{l}\text { MDC010241.225: } \\
11562-12807 \text { fwd }\end{array}$ & $\begin{array}{l}265 / 350 \\
(75.71 \%)\end{array}$ & 0.0 & Prudul26A023895T2 & $\begin{array}{c}\text { Pd08: } \\
\text { 13571383- } \\
13573631+\end{array}$ & 361/371 (97.3\%) & 0 \\
\hline PperFK1 & Prupe.2G151100.1 & $\begin{array}{c}\text { Pp02: } \\
\text { 20597751- } \\
20600680+\end{array}$ & 2 & MDP0000147281 & $\begin{array}{l}\text { MDC002232.257: } \\
6218-8755 \text { fwd }\end{array}$ & $\begin{array}{r}332 / 371 \\
(89.49 \%)\end{array}$ & 0 & Prudul26A007797T1 & $\begin{array}{c}\text { Pd02: } \\
\text { 16319571- } \\
\text { 16322428+ }\end{array}$ & $367 / 370$ (99.19\%) & 0 \\
\hline PperSUS1 & Prupe.7G192300.1 & $\begin{array}{c}\text { Pp07: } \\
\text { 18350336- } \\
\text { 18356360+ }\end{array}$ & 7 & MDP0000250070 & $\begin{array}{l}\text { MDC010818.345: } \\
28226-31888 \text { fwd }\end{array}$ & $\begin{array}{l}745 / 807 \\
(92.32 \%)\end{array}$ & 0 & Prudul26A005915T2 & $\begin{array}{c}\text { Pd07: } \\
\text { 17213855- } \\
\text { 17218941+ }\end{array}$ & $\begin{array}{r}806 / 806 \\
(100 \%)\end{array}$ & 0 \\
\hline PperSUS3 & Prupe.8G264300.1 & $\begin{array}{c}\text { Pp08: } \\
22179197- \\
22184773\end{array}$ & 8 & MDP0000739824 & $\begin{array}{l}\text { MDC013646.294: } \\
1738-4842 \text { rev }\end{array}$ & $\begin{array}{c}78 / 94 \\
(82.98 \%)\end{array}$ & $4.53 \cdot 10^{-41}$ & Prudul26A022490T1 & $\begin{array}{c}\text { Pd08: } \\
\text { 20102696- } \\
\text { 20108206- }\end{array}$ & $804 / 810(99.26 \%)$ & 0 \\
\hline PperSUS6 & Prupe.5G241700.1 & $\begin{array}{c}\text { Pp05: } \\
\text { 18195911- } \\
\text { 18200676+ }\end{array}$ & 5 & $\begin{array}{c}\text { MDC017371.127 } \\
\text { (MDP0000212593) }\end{array}$ & $\begin{array}{c}\text { MDC017371.127: } \\
\text { 11402-15390+ }\end{array}$ & $\begin{array}{l}222 / 251 \\
(88.45 \%)\end{array}$ & $\underset{134}{\substack{9.15 \cdot 10^{-} \\
134}}$ & Prudul26A027851T1 & $\begin{array}{c}\text { Pd05: } \\
\text { 17983841- } \\
\text { 17988280+ }\end{array}$ & $892 / 898$ (99.33\%) & 0 \\
\hline PperSPS1 & Prupe.7G249900.1 & $\begin{array}{c}\text { Pp07: } \\
\text { 21151882- } \\
\text { 21157785- }\end{array}$ & 7 & MDP0000331376 & $\begin{array}{l}\text { MDC018491.206: } \\
\text { 21241-23065rev }\end{array}$ & $\begin{array}{l}274 / 290 \\
(94.48 \%)\end{array}$ & $\begin{array}{c}6.82 \cdot 10^{-} \\
170\end{array}$ & Prudul26A018764T1 & $\begin{array}{c}\text { Pd07: } \\
\text { 20092219- } \\
\text { 20098144- }\end{array}$ & $\begin{array}{c}1052 / 1057 \\
(99.53 \%)\end{array}$ & 0 \\
\hline PperSPS2 & Prupe.1G483200.1 & $\begin{array}{c}\text { Pp01: } \\
\text { 40288494- } \\
\text { 40295210- }\end{array}$ & 1 & MDP0000174537 & $\begin{array}{c}\text { MDC004632.398: } \\
\text { 19489-24938 } \\
\text { reverse }\end{array}$ & $\begin{array}{c}945 / 1090 \\
(86.7 \%)\end{array}$ & 0 & Prudul26A003083T1 & $\begin{array}{c}\text { Pd01: } \\
\text { 36737186- } \\
\text { 36743693- }\end{array}$ & $\begin{array}{c}1054 / 1059 \\
(99.53 \%)\end{array}$ & 0 \\
\hline PperSPS3 & Prupe.1G159700.1 & $\begin{array}{c}\text { Pp01: } \\
\text { 12702147- } \\
\text { 12709381- }\end{array}$ & 1 & MDP0000783676 & $\begin{array}{l}\text { MDC001262.252: } \\
16461-21536 \mathrm{fwd}\end{array}$ & $\begin{array}{c}1002 / 1066 \\
(94 \%)\end{array}$ & 0 & Prudul26A023500T1 & $\begin{array}{c}\text { Pd01: } \\
\text { 12946224- } \\
\text { 12952454- }\end{array}$ & $\begin{array}{c}1062 / 1066 \\
(99.62 \%)\end{array}$ & 0 \\
\hline
\end{tabular}


Annex II

B. Prunus armeniaca against spp genomes

\begin{tabular}{|c|c|c|c|c|c|c|c|c|c|c|c|}
\hline \multirow{2}{*}{ Gene } & \multicolumn{3}{|c|}{ Prunus armeniaca } & \multicolumn{4}{|c|}{ Vitis vinifera } & \multicolumn{4}{|c|}{ Fragaria vesca } \\
\hline & Sequence ID & Position & LG & Obtained sequence & Position & Identity & Ev & Obtained sequence & Position & Identity & Ev \\
\hline ParSDH & PARG21073m09 & $\begin{array}{c}\text { LG6: } \\
\text { 7503637-7507256- }\end{array}$ & 6 & GSVIVT01010642001 & $\begin{array}{c}\text { chr16: } \\
\text { 15675424-15679159 } \\
\text { fwd }\end{array}$ & $\begin{array}{l}290 / 371 \\
(78.17 \%)\end{array}$ & 0 & $\begin{array}{c}\text { mrna13340.1-v1.0- } \\
\text { hybrid }\end{array}$ & $\begin{array}{c}\text { LG7: } \\
\text { 22062128-22064232 } \\
\text { rev }\end{array}$ & $\begin{array}{l}295 / 371 \\
(79.51 \%)\end{array}$ & 0 \\
\hline ParFK1 & PARG18141m01 & $\begin{array}{c}\text { LG5: } \\
\text { 16389523- } \\
16397626+\end{array}$ & 5 & GSVIVT01027578001 & $\begin{array}{c}\text { chr15: } \\
\text { 15399528-15404025 } \\
\text { rev }\end{array}$ & $\begin{array}{l}273 / 368 \\
(74.18 \%)\end{array}$ & $\begin{array}{c}2.34 \cdot 10^{-} \\
166\end{array}$ & $\begin{array}{c}\text { mrna19425.1-v1.0- } \\
\text { hybrid }\end{array}$ & $\begin{array}{c}\text { LG7: } \\
\text { 4380104-4385219 } \\
\text { fwd }\end{array}$ & $\begin{array}{c}45 / 61 \\
(73.77 \%)\end{array}$ & $\begin{array}{c}3.68 \cdot 10 \\
18\end{array}$ \\
\hline ParsUS1 & PARG27579m02 & $\begin{array}{c}\text { LG8: } \\
\text { 17611450- } \\
17617468+\end{array}$ & 8 & GSVIVT01015018001 & $\begin{array}{c}\text { chr11: } \\
490468-494415 \mathrm{fwd}\end{array}$ & $\begin{array}{c}669 / 806 \\
(83 \%)\end{array}$ & 0 & mrna12940.1-v1.0- & $\begin{array}{c}\text { LG1: } \\
\text { L }\end{array}$ & $\begin{array}{l}720 / 806 \\
(89.33 \%)\end{array}$ & 0 \\
\hline ParsUS3 & PARG19843m01 & $\begin{array}{c}\text { LG6: } \\
\text { 156578-162135- } \\
\text { LG7. }\end{array}$ & 6 & chr12 & $\begin{array}{c}\text { chr12: } \\
\text { 8810872-8811602+ } \\
\text { chr17: }\end{array}$ & $\begin{array}{l}19 / 25 \\
(76 \%)\end{array}$ & 1.75 & & $6534488-6537776$ rev & $\begin{array}{l}573 / 810 \\
(70.74 \%)\end{array}$ & 0 \\
\hline Parsus6 & PARG25311m01 & $\begin{array}{l}\text { 18661616- } \\
18666437-\end{array}$ & 7 & GSVIVT01029388001 & $\begin{array}{l}\text { 15994779-15999389 } \\
\text { fwd }\end{array}$ & $\begin{array}{l}455 / 781 \\
(58.26 \%)\end{array}$ & 0 & $\begin{array}{l}\text { mrna09290.1-v1.0- } \\
\text { hybrid }\end{array}$ & $\begin{array}{c}\text { LG5: } \\
\text { 9513235-9523248 rev }\end{array}$ & $\begin{array}{l}748 / 912 \\
(82.02 \%)\end{array}$ & 0 \\
\hline ParSPS1 & PARG28120m01 & $\begin{array}{c}\text { LG8: } \\
\text { 20774824- } \\
\text { 20780900- }\end{array}$ & 8 & GSVIVT01012825001 & $\begin{array}{c}\text { chr11: } \\
\text { 5766519-5776338 rev }\end{array}$ & $\begin{array}{l}878 / 1057 \\
(83.07 \%)\end{array}$ & 0 & $\begin{array}{c}\text { mrna31122.1-v1.0- } \\
\text { hybrid }\end{array}$ & $\begin{array}{c}\text { LG1: } \\
\text { 2640599-2646376 rev }\end{array}$ & $\begin{array}{l}955 / 1057 \\
(90.35 \%)\end{array}$ & 0 \\
\hline ParSPS2 & PARG08259m01 & $\begin{array}{c}\text { LG2: } \\
\text { 35365183- } \\
\text { 35371588- }\end{array}$ & 2 & GSVIVT01035882001 & $\begin{array}{c}\text { chr4: } \\
\text { 5217811-5232786 rev }\end{array}$ & $\begin{array}{l}824 / 1059 \\
(77.81 \%)\end{array}$ & 0 & $\begin{array}{c}\text { mrna11606.1-v1.0- } \\
\text { hybrid }\end{array}$ & $\begin{array}{c}\text { unanchored: } \\
\text { 9174890-9180436 } \\
\text { fwd }\end{array}$ & $\begin{array}{c}743 / 892 \\
(83.3 \%)\end{array}$ & 0 \\
\hline ParSPS3 & PARG04316m01 & $\begin{array}{c}\text { LG2: } \\
7826103-7832502\end{array}$ & 2 & GSVIVT01020928001 & $\begin{array}{c}\text { chr5: } \\
\text { 16983726-16990038 } \\
\text { rev } \\
\end{array}$ & $\begin{array}{l}800 / 975 \\
(82.05 \%)\end{array}$ & 0 & $\begin{array}{c}\text { mrna06523.1-v1.0- } \\
\text { hybrid }\end{array}$ & $\begin{array}{c}\text { LG4: } \\
\text { 16876681- } \\
\text { 16882157rev }\end{array}$ & $\begin{array}{l}891 / 976 \\
(91.29 \%)\end{array}$ & 0 \\
\hline
\end{tabular}


Table S5.B. (Cont.).

\begin{tabular}{|c|c|c|c|c|c|c|c|c|c|c|c|}
\hline \multirow{2}{*}{ Gene } & \multicolumn{3}{|c|}{ Prunus armeniaca } & \multicolumn{4}{|c|}{ Malus domestica } & \multicolumn{4}{|c|}{ Prunus dulcis } \\
\hline & Sequence ID & Position & LG & Obtained sequence & Position & Identity & Ev & Obtained sequence & Position & Identity & Ev \\
\hline ParSDH & PARG21073m09 & $\begin{array}{c}\text { LG6: } \\
\text { 7503637-7507256- }\end{array}$ & 6 & MDP0000188052 & $\begin{array}{l}\text { MDC010241.225: } \\
\text { 11562-12807 } \\
\text { fwd }\end{array}$ & $\begin{array}{l}270 / 371 \\
(72.78 \%)\end{array}$ & $8.50 \cdot 10^{-174}$ & Prudul26A023895T2 & $\begin{array}{c}\text { Pd08: } \\
\text { 13571383-13573631+ }\end{array}$ & $359 / 371$ (96.77\%) & 0 \\
\hline ParFK1 & PARG18141m01 & $\begin{array}{c}\text { LG5: } \\
16389523- \\
16397626+\end{array}$ & 5 & MDP0000147281 & $\begin{array}{l}\text { MDC002232.257: } \\
6218-8755 \text { fwd }\end{array}$ & $\begin{array}{l}326 / 366 \\
(89.07 \%)\end{array}$ & 0 & Prudul26A007797T1 & $\begin{array}{c}\text { Pd02: } \\
\text { 16319571-16322428+ }\end{array}$ & $358 / 365$ (98.08\%) & 0 \\
\hline ParsUS1 & PARG27579m02 & $\begin{array}{c}\text { LG8: } \\
\text { 17611450- } \\
17617468+\end{array}$ & 8 & MDP0000250070 & $\begin{array}{l}\text { MDC010818.345: } \\
\text { 28226-31888 } \\
\text { fwd }\end{array}$ & $\begin{array}{l}745 / 807 \\
(92.32 \%)\end{array}$ & 0 & Prudul26A005915T2 & $\begin{array}{c}\text { Pd07: } \\
\text { 17213855-17218941+ }\end{array}$ & $804 / 806$ (99.75\%) & 0 \\
\hline ParsUS3 & PARG19843m01 & $\begin{array}{c}\text { LG6: } \\
\text { 156578-162135- }\end{array}$ & 6 & MDP0000287311 & $\begin{array}{l}\text { MDC010779.409: } \\
\text { 5311-10299 rev }\end{array}$ & 78/94 (82.98\%) & $5.09 \cdot 10^{-41}$ & Prudul26A022490T1 & $\begin{array}{c}\text { Pd08: } \\
\text { 20102696-20108206- }\end{array}$ & $794 / 810(98.02 \%)$ & 0 \\
\hline Parsus6 & PARG25311m01 & $\begin{array}{c}\text { LG7: } \\
\text { 18661616- } \\
\text { 18666437- }\end{array}$ & 7 & $\begin{array}{c}\text { MDC017371.127 } \\
\text { (MDP0000212593) }\end{array}$ & $\begin{array}{l}\text { MDC017371.127: } \\
\text { 11402-15390+ }\end{array}$ & $\begin{array}{l}221 / 251 \\
(88.05 \%)\end{array}$ & $1.25 \cdot 10^{-132}$ & Prudul26A027851T1 & $\begin{array}{c}\text { Pd05: } \\
\text { 17983841-17988280+ }\end{array}$ & $881 / 899$ (98\%) & 0 \\
\hline ParSPS1 & PARG28120m01 & $\begin{array}{c}\text { LG8: } \\
\text { 20774824- } \\
\text { 20780900- }\end{array}$ & 8 & MDP0000331376 & $\begin{array}{l}\text { MDC018491.206: } \\
21241-23065 \text { rev }\end{array}$ & $\begin{array}{l}274 / 290 \\
(94.48 \%)\end{array}$ & $4.71 \cdot 10^{-170}$ & Prudul26A018764T1 & $\begin{array}{c}\text { Pd07: } \\
\text { 20092219-20098144- }\end{array}$ & $\begin{array}{c}1047 / 1057 \\
(99.05 \%)\end{array}$ & 0 \\
\hline ParSPS2 & PARG08259m01 & $\begin{array}{c}\text { LG2: } \\
35365183- \\
35371588-\end{array}$ & 2 & MDP0000174537 & $\begin{array}{l}\text { MDC004632.398: } \\
\text { 19489-24938 rev }\end{array}$ & $\begin{array}{l}946 / 1090 \\
(86.79 \%)\end{array}$ & 0 & Prudul26A003083T1 & $\begin{array}{c}\text { Pd01: } \\
\text { 36737186-36743693- }\end{array}$ & $\begin{array}{c}1043 / 1059 \\
(98.49 \%)\end{array}$ & 0 \\
\hline ParSPS3 & PARG04316m01 & $\begin{array}{c}\text { LG2: } \\
\text { 7826103-7832502 } \\
\end{array}$ & 2 & MDP0000783676 & $\begin{array}{c}\text { MDC001262.252: } \\
\text { 16461-21536 } \\
\text { fwd } \\
\end{array}$ & $\begin{array}{l}910 / 974 \\
(93.43 \%)\end{array}$ & 0 & Prudul26A023500T1 & $\begin{array}{c}\text { Pd01: } \\
\text { 12946224..12952454- }\end{array}$ & 968/974 (99.38\%) & 0 \\
\hline
\end{tabular}


Table S6. Gene expression. Mean, standard deviation (sd) and significance (sg)

\begin{tabular}{|c|c|c|c|c|c|c|c|c|c|c|c|c|c|c|c|c|c|c|c|c|c|c|c|c|}
\hline \multirow{2}{*}{ Genotype } & \multicolumn{3}{|c|}{ ParSDH } & \multicolumn{3}{|c|}{ ParSPS1 } & \multicolumn{3}{|c|}{ ParSPS2 } & \multicolumn{3}{|c|}{ ParSPS3 } & \multicolumn{3}{|c|}{ ParSUS1 } & \multicolumn{3}{|c|}{ ParSUS3 } & \multicolumn{3}{|c|}{ ParSUS6 } & \multicolumn{3}{|c|}{ ParFK1 } \\
\hline & Mean & sd & sg & Mean & sd & sg & Mean & sd & sg & Mean & sd & sg & Mean & sd & sg & Mean & sd & sg & Mean & sd & sg & Mean & sd & sg \\
\hline 'Canino' & 3.31 & 4.59 & $\mathrm{a}$ & 4.22 & 1.21 & $a$ & 3.77 & 1.10 & c & 2.61 & 0.88 & abcd & 4.60 & 1.54 & $\mathrm{~cd}$ & 4.35 & 0.73 & c & 4.70 & 3.99 & $\mathrm{a}$ & 5.67 & 7.72 & $a$ \\
\hline 'Dama Rosa' & 1.11 & 1.40 & $\mathrm{a}$ & 1.85 & 0.54 & $a$ & 2.05 & 0.56 & $a b$ & 4.42 & 1.52 & cde & 1.19 & 0.29 & a & 1.75 & 0.40 & a & 2.92 & 0.86 & a & 0.97 & 0.62 & a \\
\hline 'Dama Taronja' & 0.94 & 0.69 & a & 2.56 & 0.63 & $a$ & 1.93 & 0.28 & a & 1.74 & 1.13 & $a b c$ & 2.79 & 0.74 & abcd & 1.89 & 0.32 & $a b$ & 1.22 & 0.55 & $a$ & 0.48 & 0.24 & $a$ \\
\hline 'GG9310' & 1.01 & 1.49 & $\mathrm{a}$ & 3.15 & 1.69 & $a$ & 2.55 & 1.12 & $a b c$ & 2.54 & 0.61 & abcd & 2.04 & 0.71 & $a b$ & 1.99 & 0.37 & $a b$ & 2.92 & 1.83 & a & 0.78 & 1.04 & $a$ \\
\hline 'GG979' & 0.40 & 0.21 & $\mathrm{a}$ & 2.79 & 1.06 & $a$ & 2.38 & 0.71 & $a b$ & 1.33 & 0.71 & $a b c$ & 4.00 & 1.14 & bcd & 3.22 & 0.23 & $a b c$ & 1.10 & 0.55 & $a$ & 0.19 & 0.12 & a \\
\hline 'Goldrich' & 0.59 & 0.45 & $a$ & 2.52 & 0.81 & $a$ & 1.96 & 0.42 & a & 0.70 & 0.41 & $a b$ & 4.80 & 3.02 & $d$ & 3.24 & 1.70 & $a b c$ & 0.94 & 0.42 & $a$ & 0.98 & 0.85 & a \\
\hline 'GP9817’ & 1.79 & 2.20 & a & 2.04 & 0.68 & $\mathrm{a}$ & 1.46 & 0.65 & a & 3.23 & 0.85 & abcde & 1.27 & 0.24 & a & 1.61 & 0.80 & a & 1.90 & 1.13 & $a$ & 0.85 & 0.63 & a \\
\hline 'HG9821' & 2.77 & 3.22 & a & 2.50 & 0.35 & $a$ & 2.62 & 0.37 & $a b c$ & 5.29 & 2.30 & de & 2.86 & 0.64 & abcd & 2.49 & 0.76 & $a b$ & 5.59 & 3.54 & $a$ & 1.91 & 1.57 & $a$ \\
\hline 'HG9850’ & 2.22 & 1.99 & a & 2.87 & 0.55 & $a$ & 2.52 & 0.70 & $a b c$ & 6.06 & 2.62 & e & 3.16 & 0.48 & abcd & 3.20 & 0.82 & $a b c$ & 5.58 & 2.61 & a & 1.53 & 1.64 & a \\
\hline 'HM964’ & 2.43 & 1.91 & a & 2.92 & 1.38 & $a$ & 2.65 & 0.42 & $a b c$ & 3.96 & 2.89 & bcde & 1.53 & 0.39 & a & 2.75 & 1.15 & $a b c$ & 5.08 & 2.04 & $\mathrm{a}$ & 2.37 & 2.06 & $a$ \\
\hline 'Mitger' & 0.19 & 0.46 & $a$ & 2.91 & 1.17 & $a$ & 3.28 & 0.51 & bc & 0.62 & 0.25 & $a$ & 1.77 & 0.35 & $a b$ & 3.65 & 1.05 & bc & 5.03 & 3.93 & a & 0.37 & 0.32 & a \\
\hline 'SEOP934' & 1.20 & 1.35 & $\mathrm{a}$ & 2.95 & 0.93 & $\mathrm{a}$ & 1.95 & 0.29 & a & 4.19 & 1.99 & cde & 2.32 & 0.64 & $a b c$ & 2.56 & 0.83 & $a b$ & 3.86 & 1.53 & $\mathrm{a}$ & 1.27 & 1.45 & a \\
\hline
\end{tabular}


Table S7. Polyphenol total concentration in flesh (mg/100g FW) and peel (mg/100g DW) for each year and three years average (mean \pm standard deviation). Different letter means significant differences among genotypes.

\begin{tabular}{llllll}
\hline Genotype & Flesh & Peel & & & \\
\cline { 2 - 6 } & $\mathbf{2 0 1 6}$ & $\mathbf{2 0 1 6}$ & $\mathbf{2 0 1 7}$ & $\mathbf{2 0 1 8}$ & $\mathbf{3}$ years average \\
\hline 'Canino' & 46.00 & 706.37 & 1080.5 & 547.75 & $778.21 \pm 273.55 \mathrm{abc}$ \\
'Dama Rosa' & 57.52 & 986.07 & 893.58 & 749.58 & $876.41 \pm 119.18 \mathrm{bc}$ \\
'Dama Taronja' & 44.70 & 994.13 & 694.06 & 416.78 & $701.66 \pm 288.75 \mathrm{ab}$ \\
'GG9310' & 91.29 & 734.34 & 684.94 & 745.11 & $721.46 \pm 32.08 \mathrm{abc}$ \\
'GG979' & 39.19 & 773.07 & 496.54 & 584.31 & $617.98 \pm 141.3 \mathrm{ab}$ \\
'Goldrich' & 97.41 & 1196.41 & 1228.1 & 706.07 & $1043.53 \pm 292.69 \mathrm{c}$ \\
'GP9817' & 96.62 & 821.95 & 939.37 & 569.57 & $776.96 \pm 188.96 \mathrm{abc}$ \\
'HG9821' & 67.69 & 659.43 & 440.76 & 794.67 & $631.62 \pm 178.59 \mathrm{ab}$ \\
'HG9850' & 41.47 & 552.18 & 461.3 & 495.42 & $502.97 \pm 45.91 \mathrm{a}$ \\
'HM964' & 61.32 & 697.37 & 1001.8 & 537.14 & $745.44 \pm 236.04 \mathrm{abc}$ \\
'Mitger' & 41.60 & 903.77 & 456.58 & 500.72 & $620.36 \pm 246.43 \mathrm{ab}$ \\
'SEOP934' & 40.21 & 652.64 & 880.11 & 764.89 & $765.88 \pm 113.74 \mathrm{abc}$ \\
'Tadeo' & 38.05 & 729.44 & 835.53 & 561.18 & $708.72 \pm 138.35 \mathrm{ab}$ \\
\hline
\end{tabular}

Table S8. Neochlorogenic acid concentration in peel (mg/100g DW) in 2016, 2017, 2018 and three years average. Mean \pm standard deviation. Different letter means significant differences.

\begin{tabular}{lllll}
\hline Genotype & \multicolumn{1}{c}{ 3 years average } & \multicolumn{1}{c}{$\mathbf{2 0 1 6}$} & \multicolumn{1}{c}{$\mathbf{2 0 1 7}$} & \multicolumn{1}{c}{$\mathbf{2 0 1 8}$} \\
\hline 'Canino' & $174.43 \pm 53.13 \mathrm{abc}$ & $209.08 \pm 14.9 \mathrm{cde}$ & $200.95 \pm 16.12 \mathrm{c}$ & $113.27 \pm 3.16 \mathrm{ab}$ \\
'Dama Rosa' & $241.75 \pm 68.33 \mathrm{bcd}$ & $229.65 \pm 13.81 \mathrm{def}$ & $315.32 \pm 15.95 \mathrm{ef}$ & $180.27 \pm 12.83 \mathrm{~g}$ \\
'Dama Taronja' & $241.75 \pm 83.66 \mathrm{bcd}$ & $267.75 \pm 14.84 \mathrm{f}$ & $286.22 \pm 30.11 \mathrm{~d}$ & $132.97 \pm 15.16 \mathrm{c}$ \\
'GG9310' & $271.33 \pm 49.16 \mathrm{~cd}$ & $244.84 \pm 22.49 \mathrm{ef}$ & $328.05 \pm 12.84 \mathrm{f}$ & $241.11 \pm 7.37 \mathrm{~h}$ \\
'GG979' & $160.31 \pm 19.75 \mathrm{ab}$ & $165.22 \pm 7.36 \mathrm{bc}$ & $177.14 \pm 16.22 \mathrm{bc}$ & $138.56 \pm 1.13 \mathrm{~cd}$ \\
'Goldrich' & $299.97 \pm 106.69 \mathrm{~d}$ & $357.99 \pm 91.07 \mathrm{~g}$ & $365.08 \pm 12.92 \mathrm{~g}$ & $176.85 \pm 4.78 \mathrm{fg}$ \\
'GP9817' & $236.79 \pm 73.99 \mathrm{bcd}$ & $261.39 \pm 24.77 \mathrm{f}$ & $295.36 \pm 9.04 \mathrm{de}$ & $153.63 \pm 3.19 \mathrm{de}$ \\
'HG9821' & $162.66 \pm 16.52 \mathrm{abc}$ & $145.17 \pm 5.89 \mathrm{ab}$ & $164.81 \pm 0.33 \mathrm{~b}$ & $178 \pm 19.08 \mathrm{~g}$ \\
'HG9850' & $113.32 \pm 12.43 \mathrm{a}$ & $102.14 \pm 14.26 \mathrm{a}$ & $126.71 \pm 21.21 \mathrm{a}$ & $111.11 \pm 5.99 \mathrm{a}$ \\
'HM964' & $237.58 \pm 109.86 \mathrm{bcd}$ & $186.09 \pm 12.56 \mathrm{bcd}$ & $363.73 \pm 19.53 \mathrm{~g}$ & $162.92 \pm 1.01 \mathrm{ef}$ \\
'Mitger' & $164.16 \pm 38 \mathrm{abc}$ & $203.47 \pm 16.23 \mathrm{cde}$ & $161.38 \pm 10.13 \mathrm{~b}$ & $127.63 \pm 0.32 \mathrm{bc}$ \\
'SEOP934' & $207.65 \pm 88.31 \mathrm{abcd}$ & $164.63 \pm 11.56 \mathrm{bc}$ & $309.22 \pm 15.82 \mathrm{def}$ & $149.08 \pm 12.41 \mathrm{de}$ \\
'Tadeo' & $139.32 \pm 20.76 \mathrm{ab}$ & $162.97 \pm 1.36 \mathrm{bc}$ & $124.07 \pm 8.45 \mathrm{a}$ & $130.93 \pm 1.35 \mathrm{c}$ \\
\hline
\end{tabular}


Table S9. Chlorogenic acid concentration in peel (mg/100g DW) in 2016, 2017, 2018, and three years average. Mean \pm standard deviation. Different letter means significant.

\begin{tabular}{l|llll}
\hline Genotype & \multicolumn{1}{c}{$\mathbf{3}$ years average } & \multicolumn{1}{c}{$\mathbf{2 0 1 6}$} & \multicolumn{1}{c}{$\mathbf{2 0 1 7}$} & \multicolumn{1}{c}{$\mathbf{2 0 1 8}$} \\
\hline 'Canino' & $110.28 \pm 38.94 \mathrm{a}$ & $152.35 \pm 8.24 \mathrm{abc}$ & $102.98 \pm 12.52 \mathrm{a}$ & $75.50 \pm 4.09 \mathrm{a}$ \\
'Dama Rosa' & $262.96 \pm 117.32 \mathrm{bc}$ & $262.21 \pm 14.78 \mathrm{e}$ & $380.66 \pm 17.02 \mathrm{~g}$ & $146.03 \pm 6.02 \mathrm{gh}$ \\
'Dama Taronja' & $125.27 \pm 51.23 \mathrm{a}$ & $139.36 \pm 27.61 \mathrm{ab}$ & $167.99 \pm 18.79 \mathrm{c}$ & $68.47 \pm 8.78 \mathrm{a}$ \\
'GG9310' & $132.04 \pm 15.69 \mathrm{a}$ & $141.08 \pm 16.70 \mathrm{ab}$ & $141.12 \pm 7.01 \mathrm{bc}$ & $113.92 \pm 2.12 \mathrm{c}$ \\
'GG979' & $165.08 \pm 31.4 \mathrm{abc}$ & $165.78 \pm 6.22 \mathrm{bc}$ & $196.12 \pm 19.22 \mathrm{~d}$ & $133.33 \pm 5.19 \mathrm{ef}$ \\
'Goldrich' & $277.13 \pm 129.46 \mathrm{c}$ & $250.69 \pm 67.79 \mathrm{e}$ & $417.77 \pm 30.89 \mathrm{~h}$ & $162.94 \pm 6.14 \mathrm{i}$ \\
'GP9817' & $197.69 \pm 121.98 \mathrm{abc}$ & $126.04 \pm 8.95 \mathrm{a}$ & $338.54 \pm 9.09 \mathrm{f}$ & $128.50 \pm 2.60 \mathrm{de}$ \\
'HG9821' & $126.27 \pm 31.78 \mathrm{a}$ & $130.05 \pm 7.29 \mathrm{ab}$ & $92.77 \pm 0.40 \mathrm{a}$ & $156.00 \pm 9.17 \mathrm{hi}$ \\
'HG9850' & $136.91 \pm 11.99 \mathrm{ab}$ & $123.09 \pm 17.26 \mathrm{a}$ & $144.52 \pm 31.72 \mathrm{bc}$ & $143.14 \pm 10.92 \mathrm{fg}$ \\
'HM964' & $203.72 \pm 92.04 \mathrm{abc}$ & $189.11 \pm 10.90 \mathrm{~cd}$ & $302.19 \pm 4.45 \mathrm{e}$ & $119.87 \pm 2.01 \mathrm{~cd}$ \\
'Mitger' & $134.59 \pm 61.62 \mathrm{ab}$ & $205.73 \pm 18.55 \mathrm{~d}$ & $98.04 \pm 3.34 \mathrm{a}$ & $100.00 \pm 1.96 \mathrm{~b}$ \\
'SEOP934' & $224.15 \pm 107.9 \mathrm{abc}$ & $181.01 \pm 3.48 \mathrm{~cd}$ & $346.94 \pm 15.93 \mathrm{f}$ & $144.48 \pm 15.58 \mathrm{fgh}$ \\
'Tadeo' & $123.23 \pm 29.97 \mathrm{a}$ & $155.75 \pm 5.08 \mathrm{abc}$ & $117.25 \pm 3.40 \mathrm{ab}$ & $96.71 \pm 1.16 \mathrm{~b}$ \\
\hline
\end{tabular}

Table S10. Rutin concentration in peel (mg/100g DW) in 2016, 2017, 2018, and three years average. Mean \pm standard deviation. Different letter means significant differences.

\begin{tabular}{lllll}
\hline Genotype & Mean & Mean 2016 & Mean 2017 & Mean 2018 \\
\hline 'Canino' & $420.16 \pm 238.55 \mathrm{a}$ & $264.34 \pm 22.56 \mathrm{abc}$ & $694.79 \pm 48.06 \mathrm{~d}$ & $301.36 \pm 4.57 \mathrm{efg}$ \\
'Dama Rosa' & $314.44 \pm 132.14 \mathrm{a}$ & $419.20 \pm 21.12 \mathrm{ef}$ & $165.99 \pm 21.02 \mathrm{a}$ & $358.13 \pm 31.08 \mathrm{hi}$ \\
'Dama & $272.34 \pm 167.14 \mathrm{a}$ & $464.42 \pm 31.81 \mathrm{f}$ & $192.54 \pm 26.85 \mathrm{a}$ & $160.05 \pm 24.69 \mathrm{a}$ \\
'GG9310' & $264.56 \pm 73.86 \mathrm{a}$ & $285.30 \pm 20.58 \mathrm{abc}$ & $182.54 \pm 5.18 \mathrm{a}$ & $325.83 \pm 2.02 \mathrm{gh}$ \\
'GG979' & $241.45 \pm 134.84 \mathrm{a}$ & $367.16 \pm 16.55 \mathrm{de}$ & $99.03 \pm 20.70 \mathrm{a}$ & $258.17 \pm 16.44 \mathrm{cde}$ \\
'Goldrich' & $387.82 \pm 86.87 \mathrm{a}$ & $478.48 \pm 118.71 \mathrm{f}$ & $379.65 \pm 120.37 \mathrm{~cd}$ & $305.32 \pm 7.58 \mathrm{fg}$ \\
'GP9817' & $293.97 \pm 67.3 \mathrm{a}$ & $369.29 \pm 22.87 \mathrm{de}$ & $272.88 \pm 30.38 \mathrm{bc}$ & $239.75 \pm 9.26 \mathrm{bcd}$ \\
'HG9821' & $289.51 \pm 117.55 \mathrm{a}$ & $323.02 \pm 20.06 \mathrm{bcd}$ & $158.85 \pm 2.77 \mathrm{a}$ & $386.67 \pm 48.01 \mathrm{i}$ \\
'HG9850' & $219.05 \pm 42.15 \mathrm{a}$ & $257.61 \pm 32.71 \mathrm{ab}$ & $174.06 \pm 29.21 \mathrm{a}$ & $225.49 \pm 20.75 \mathrm{bc}$ \\
'HM964' & $243.43 \pm 46.39 \mathrm{a}$ & $248.43 \pm 16.64 \mathrm{a}$ & $287.11 \pm 20.29 \mathrm{ab}$ & $194.73 \pm 9.82 \mathrm{ab}$ \\
'Mitger' & $268.43 \pm 130.97 \mathrm{a}$ & $415.74 \pm 32.31 \mathrm{ef}$ & $165.15 \pm 12.68 \mathrm{a}$ & $224.39 \pm 21.62 \mathrm{bc}$ \\
'SEOP934' & $255.74 \pm 71.7 \mathrm{a}$ & $222.04 \pm 7.86 \mathrm{a}$ & $207.09 \pm 5.56 \mathrm{a}$ & $338.08 \pm 65.30 \mathrm{gh}$ \\
'Tadeo' & $375.03 \pm 127.49 \mathrm{a}$ & $329.83 \pm 8.58 \mathrm{~cd}$ & $518.97 \pm 89.52 \mathrm{~cd}$ & $276.30 \pm 10.97 \mathrm{def}$ \\
\hline
\end{tabular}


Table S11. Quercetin-3-glucuronide content in peel (mg/100gDW) in 2016, 2017, 2018 and three years average. Mean \pm standard deviation. Different letter means significant differences.

\begin{tabular}{lllll}
\hline Genotype & 3 years average & Mean 2016 & Mean 2017 & Mean 2018 \\
\hline 'Canino' & $73.34 \pm 13.62 \mathrm{a}$ & $80.60 \pm 4.12 \mathrm{~cd}$ & $81.80 \pm 12.10 \mathrm{~g}$ & $57.62 \pm 0.66 \mathrm{bc}$ \\
'Dama Rosa' & $57.26 \pm 22.74 \mathrm{a}$ & $75.01 \pm 1.14 \mathrm{abcd}$ & $31.62 \pm 2.05 \mathrm{~cd}$ & $65.15 \pm 2.66 \mathrm{~cd}$ \\
'Dama Taronja' & $75.06 \pm 41.35 \mathrm{a}$ & $122.59 \pm 6.24 \mathrm{e}$ & $47.31 \pm 4.20 \mathrm{e}$ & $55.29 \pm 4.52 \mathrm{bc}$ \\
'GG9310' & $53.53 \pm 17.59 \mathrm{a}$ & $63.12 \pm 4.98 \mathrm{ab}$ & $33.22 \pm 0.57 \mathrm{~d}$ & $64.25 \pm 1.64 \mathrm{~cd}$ \\
'GG979' & $51.14 \pm 25.47 \mathrm{a}$ & $74.91 \pm 1.86 \mathrm{abcd}$ & $24.26 \pm 1.82 \mathrm{bc}$ & $54.25 \pm 1.13 \mathrm{bc}$ \\
'Goldrich' & $78.61 \pm 26.63 \mathrm{a}$ & $109.24 \pm 30.68 \mathrm{e}$ & $65.62 \pm 4.14 \mathrm{f}$ & $60.95 \pm 3.68 \mathrm{bcd}$ \\
'GP9817' & $48.5 \pm 16.33 \mathrm{a}$ & $65.23 \pm 5.33 \mathrm{abc}$ & $32.60 \pm 1.10 \mathrm{~d}$ & $47.69 \pm 0.54 \mathrm{~b}$ \\
'HG9821' & $53.17 \pm 25.79 \mathrm{a}$ & $61.19 \pm 3.97 \mathrm{a}$ & $24.33 \pm 0.58 \mathrm{bc}$ & $74.00 \pm 6.00 \mathrm{~d}$ \\
'HG9850' & $33.68 \pm 30.88 \mathrm{a}$ & $69.34 \pm 8.38 \mathrm{abcd}$ & $16.02 \pm 0.85 \mathrm{a}$ & $15.69 \pm 3.92 \mathrm{a}$ \\
'HM964' & $60.71 \pm 12.51 \mathrm{a}$ & $73.73 \pm 5.56 \mathrm{abcd}$ & $48.79 \pm 4.83 \mathrm{e}$ & $59.62 \pm 2.59 \mathrm{bc}$ \\
'Mitger' & $53.18 \pm 23.73 \mathrm{a}$ & $78.82 \pm 4.75 \mathrm{bcd}$ & $32.01 \pm 5.03 \mathrm{~d}$ & $48.69 \pm 3.15 \mathrm{~b}$ \\
'SEOP934' & $78.35 \pm 58.47 \mathrm{a}$ & $84.96 \pm 4.58 \mathrm{~d}$ & $16.85 \pm 0.74 \mathrm{ab}$ & $133.24 \pm 28.54 \mathrm{e}$ \\
'Tadeo' & $71.13 \pm 12.35 \mathrm{a}$ & $80.89 \pm 5.54 \mathrm{~cd}$ & $75.25 \pm 5.40 \mathrm{~g}$ & $57.24 \pm 0.66 \mathrm{bc}$ \\
\hline
\end{tabular}

Table S12. Prunus persica and Prunus armeniaca synteny and protein identity.

\begin{tabular}{|c|c|c|c|c|c|c|c|c|}
\hline \multirow{2}{*}{$\begin{array}{l}\text { Gene } \\
\text { name }\end{array}$} & \multicolumn{3}{|c|}{ Prunus persica } & \multicolumn{2}{|c|}{ Prunus armeniaca } & \multicolumn{3}{|c|}{$\begin{array}{l}\text { Prunus persica vs Prunus } \\
\text { armeniaca }\end{array}$} \\
\hline & $\begin{array}{l}\text { Sequence } \\
\text { identifier }\end{array}$ & Gene location & LG & $\begin{array}{l}\text { Obtained } \\
\text { sequence }\end{array}$ & Position & $\begin{array}{l}\text { Synteny } \\
\text { block }\end{array}$ & Identity & $\begin{array}{c}\text { E- } \\
\text { value }\end{array}$ \\
\hline PpePAL1 & Prupe.2G211800.1 & $\begin{array}{c}\text { Pp02:24393791- } \\
24397929\end{array}$ & 2 & PARG18722 & $\begin{array}{c}\text { LG5:20435326- } \\
20438772\end{array}$ & арppB272 & $\begin{array}{l}710 / 719 \\
(98.75 \%)\end{array}$ & 0 \\
\hline PpePAL2 & Prupe.6G235400.1 & $\begin{array}{c}\text { Pp06:23639324- } \\
23642649\end{array}$ & 6 & PARG02214 & $\begin{array}{c}\text { LG1:17635804- } \\
17638656\end{array}$ & арppB018 & $\begin{array}{l}711 / 717 \\
(99.16 \%)\end{array}$ & 0 \\
\hline PpeDFR & Prupe.1G376400.1 & $\begin{array}{c}\text { Pp01:34110835- } \\
34113473\end{array}$ & 1 & PARG07267 & $\begin{array}{c}\text { LG2: } 29288431- \\
29290653\end{array}$ & аррpB056 & $\begin{array}{l}333 / 346 \\
(96.24 \%)\end{array}$ & 0 \\
\hline PpeFLS1 & Prupe.1G502700.1 & $\begin{array}{c}\text { Pp01:41577480- } \\
41579444\end{array}$ & 1 & PARG08425 & $\begin{array}{c}\text { LG2:36614060- } \\
36616498\end{array}$ & аррpB057 & $\begin{array}{l}328 / 335 \\
(97.91 \%)\end{array}$ & 0 \\
\hline PpeFLS2 & Prupe.1G502800.1 & $\begin{array}{c}\text { Pp01:41580633- } \\
41583079\end{array}$ & 1 & PARG08426 & $\begin{array}{c}\text { LG2:36622560- } \\
36624610\end{array}$ & арррв057 & $\begin{array}{l}330 / 338 \\
(97.63 \%)\end{array}$ & 0 \\
\hline
\end{tabular}


Table S13. Arabidopsis thaliana and Prunus armeniaca protein identity.

\begin{tabular}{l|cc|cc}
\hline \multirow{2}{*}{\begin{tabular}{c} 
Arabidopsis \\
\multicolumn{1}{c}{ thaliana }
\end{tabular}} & Prunus armeniaca & $\begin{array}{c}\text { Arabidopsis thaliana vs Prunus } \\
\text { persica }\end{array}$ \\
\cline { 2 - 5 } AtPAL1 & ParPAL2 & PARG02214m01 & $591 / 705(83.83 \%)$ & 0 \\
AtPAL2 & Identity with apricot & E-value \\
AtPAL3 & ParPAL1 & PARG18722m01 & $576 / 692(83.24 \%)$ & 0 \\
AtPAL4 & ParPAL1 & PARG18722m01 & $579 / 709(81.66 \%)$ & 0 \\
AtDFR & ParDFR & PARG07267m01 & $241 / 327(73.7 \%)$ & $1.15 \cdot 10^{-161}$ \\
AtFLS1 & ParFLS1 & PARG08425m01 & $200 / 333(60.06 \%)$ & $3.35 \cdot 10^{-126}$ \\
AtFLS2 & ParFLS2 & PARG08426m01 & $105 / 230(45.65 \%)$ & $2.38 \cdot 10^{-50}$ \\
AtFLS3 & ParFLS1 & PARG08425m01 & $158 / 294(53.74 \%)$ & $3.23 \cdot 10^{-94}$ \\
\hline
\end{tabular}


Table S14. p-distance for PAL (A), DFR (B) and FLS (C ) proteins.

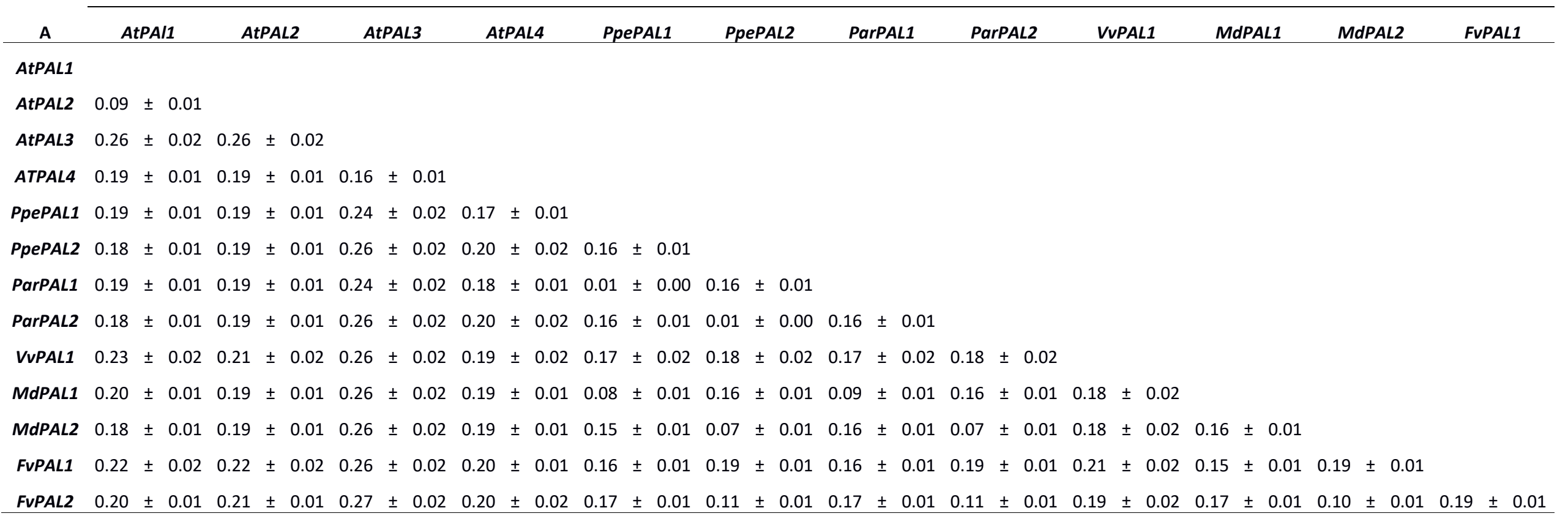

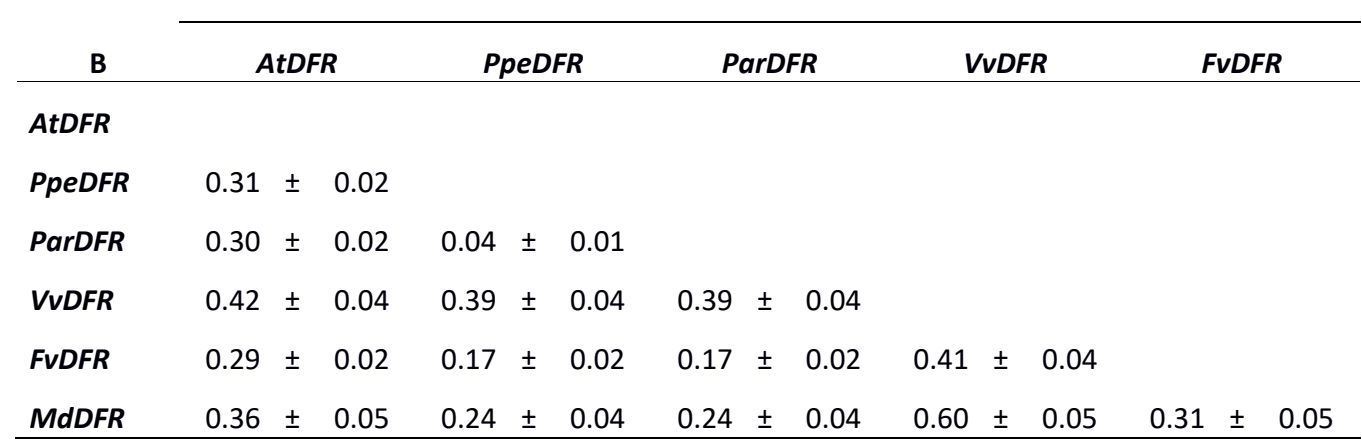




\begin{tabular}{|c|c|c|c|c|c|c|c|c|c|c|}
\hline C & ATFLS1 & AtFLS2 & AtFLS3 & AtFLS3 & PpeFLS1 & PpeFLS2 & ParFLS1 & ParFLS2 & VvFLS1 & MdFLS1 \\
\hline \multicolumn{11}{|l|}{ AtFLS1 } \\
\hline AtFLS2 & $0.3 \pm 0.0$ & & & & & & & & & \\
\hline AtFLS3 & $0.3 \pm 0.0$ & $0.4 \pm 0.0$ & & & & & & & & \\
\hline PpeFLS1 & $0.4 \pm 0.0$ & $0.5 \pm 0.0$ & $0.4 \pm 0.0$ & & & & & & & \\
\hline PpeFLS2 & $0.4 \pm 0.0$ & $0.5 \pm 0.0$ & $0.4 \pm 0.0$ & $0.2 \pm 0.0$ & & & & & & \\
\hline ParFLS1 & $0.4 \pm 0.0$ & $0.5 \pm 0.0$ & $0.4 \pm 0.0$ & $0.0 \pm 0.0$ & $0.2 \pm 0.0$ & & & & & \\
\hline ParfLS2 & $0.4 \pm 0.0$ & $0.5 \pm 0.0$ & $0.4 \pm 0.0$ & $0.2 \pm 0.0$ & $0.0 \pm 0.0$ & $0.2 \pm 0.0$ & & & & \\
\hline VvFLS1 & $0.3 \pm 0.0$ & $0.5 \pm 0.0$ & $0.5 \pm 0.0$ & $0.2 \pm 0.0$ & $0.2 \pm 0.0$ & $0.3 \pm 0.0$ & $0.2 \pm 0.0$ & & & \\
\hline FvFLS1 & $0.4 \pm 0.0$ & $0.5 \pm 0.0$ & $0.4 \pm 0.0$ & $0.2 \pm 0.0$ & $0.2 \pm 0.0$ & $0.2 \pm 0.0$ & $0.2 \pm 0.0$ & $0.2 \pm 0.0$ & & \\
\hline MdFLS1 & $0.4 \pm 0.0$ & $0.5 \pm 0.0$ & $0.5 \pm 0.0$ & $0.2 \pm 0.0$ & $0.3 \pm 0.0$ & $0.2 \pm 0.0$ & $0.3 \pm 0.0$ & $0.3 \pm 0.0$ & $0.2 \pm 0.0$ & \\
\hline MdFLS2 & $0.6 \pm 0.0$ & $0.7 \pm 0.0$ & $0.6 \pm 0.0$ & $0.5 \pm 0.0$ & $0.4 \pm 0.0$ & $0.5 \pm 0.0$ & $0.4 \pm 0.0$ & $0.6 \pm 0.0$ & $0.5 \pm 0.0$ & $0.3 \pm 0.0$ \\
\hline
\end{tabular}


Table S15. Similarity (1-p-distance) among protein sequences of PAL (A), DFR (B), FLS (C).

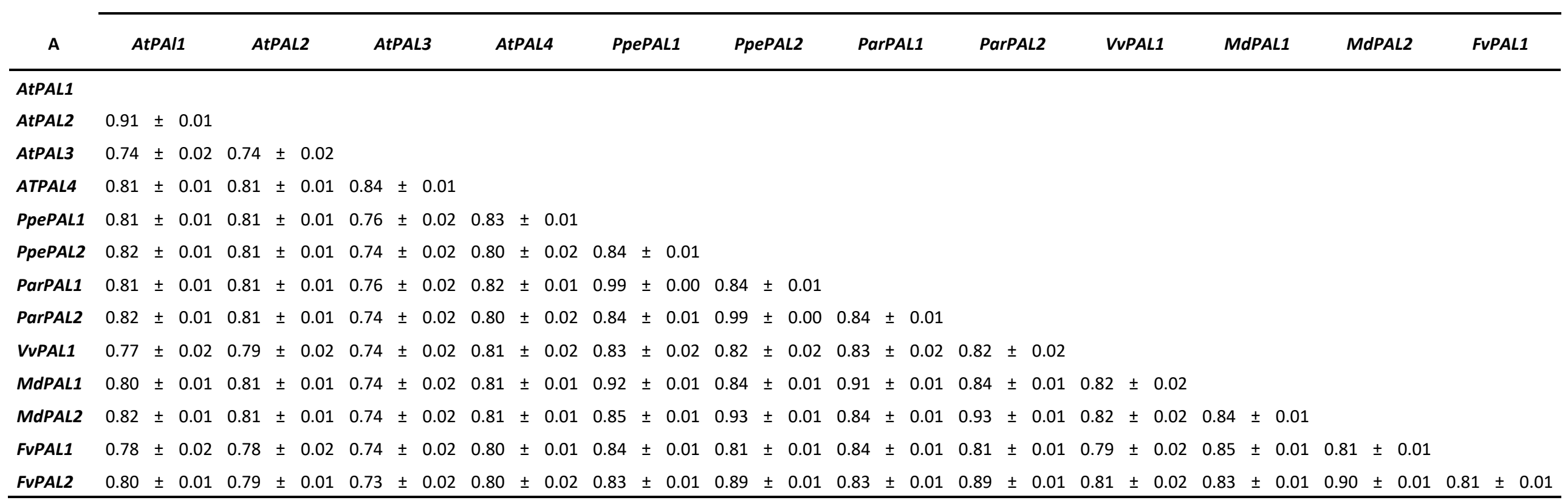

\begin{tabular}{|c|c|c|c|c|c|c|c|c|c|c|c|c|c|c|c|}
\hline \multirow{2}{*}{$\frac{\text { B }}{\text { AtDFR }}$} & \multicolumn{3}{|c|}{ AtDFR } & \multicolumn{3}{|c|}{ PpeDFR } & \multicolumn{3}{|c|}{ ParDFR } & \multicolumn{3}{|c|}{ VvDFR } & \multicolumn{3}{|c|}{ FvDFR } \\
\hline & & & & & & & & & & & & & & & \\
\hline PpeDFR & 0.69 & \pm & 0.02 & & & & & & & & & & & & \\
\hline ParDFR & 0.70 & \pm & 0.02 & 0.96 & $5 \pm$ & 0.01 & & & & & & & & & \\
\hline VVDFR & 0.58 & \pm & 0.04 & 0.61 & $1 \pm$ & 0.04 & 0.61 & $1 \pm$ & 0.04 & & & & & & \\
\hline FvDFR & 0.71 & \pm & 0.02 & 0.83 & $3 \pm$ & 0.02 & 0.83 & $3 \pm$ & 0.02 & 0.59 & $9 \pm$ & 0.04 & & & \\
\hline MdDFR & 0.64 & \pm & 0.05 & 0.76 & $5 \pm$ & 0.04 & 0.76 & $6 \pm$ & 0.04 & 0.40 & $0 \pm$ & 0.05 & 0.69 & \pm & 0.05 \\
\hline
\end{tabular}




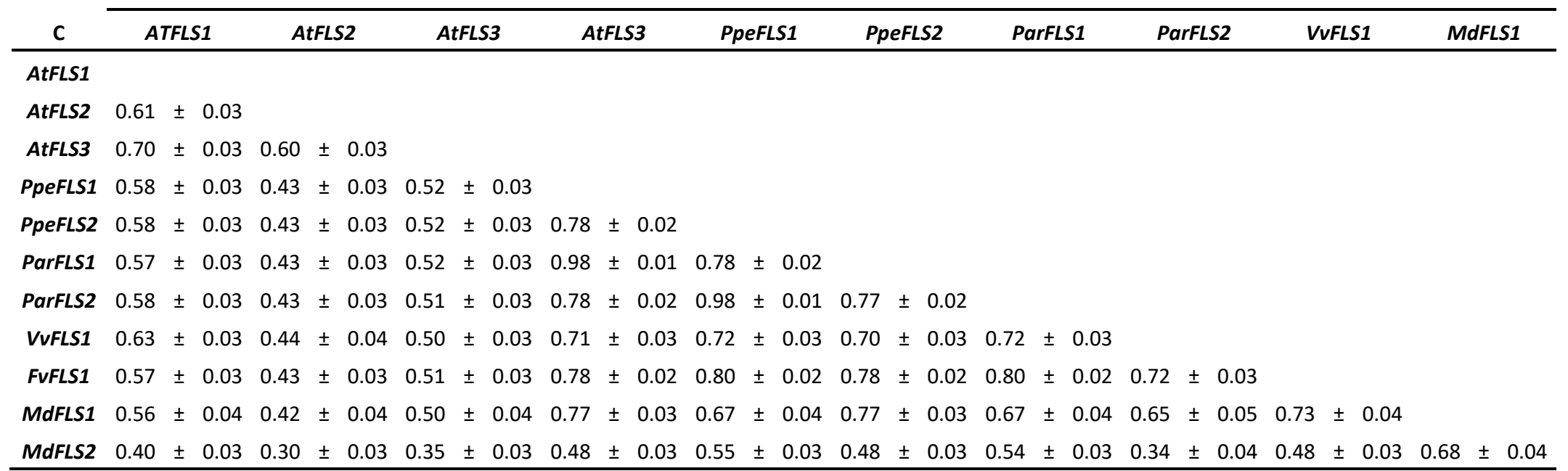


Table S16. Genetic expression of studied genotypes. Different letter means significant differences among genotypes.

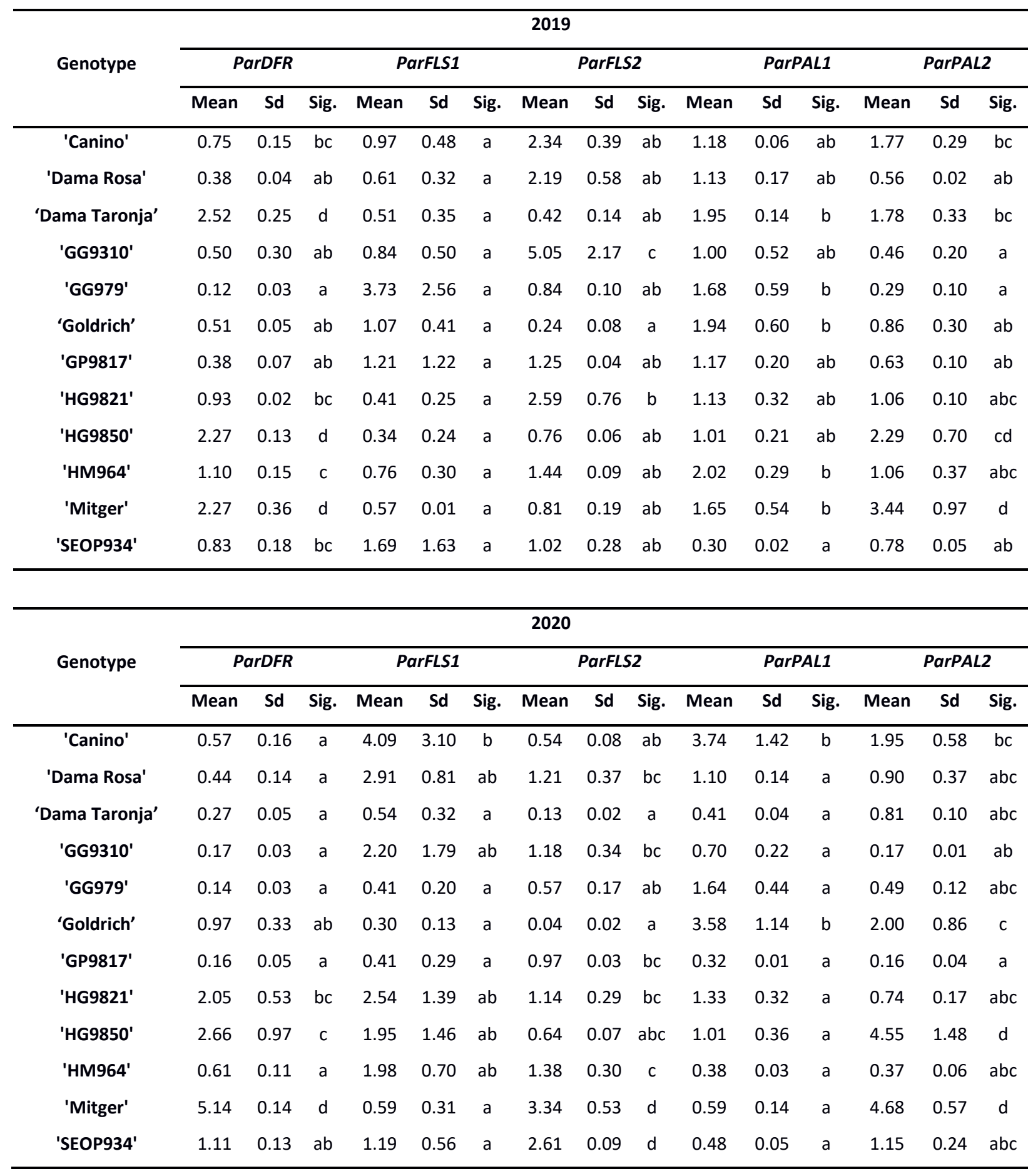


Table S17. Linear regression model in caffeate-derivates.

\begin{tabular}{|c|c|c|c|c|c|}
\hline \multicolumn{6}{|c|}{ NEOCHLORGENIC ACID } \\
\hline \multicolumn{6}{|c|}{2019} \\
\hline Parameter & Estimation & Sd & $\mathbf{T}$ & P-value & $\mathbf{R 2}$ \\
\hline CONSTANT & 185.653 & 42.8202 & 4.33564 & 0.0002 & \\
\hline ParDFR & -81.7058 & 33.5748 & -2.43355 & 0.0216 & \\
\hline ParFLS2 & 34.5919 & 10.6549 & 3.24656 & 0.003 & \\
\hline ParPAL1 & 106.167 & 33.3428 & 3.1841 & 0.0035 & 0.6191 \\
\hline ParPAL2 & -108.959 & 36.882 & -2.95426 & 0.0063 & \\
\hline ParPAL1Par / FLS2 & -49.3894 & 19.9979 & -2.46973 & 0.0199 & \\
\hline ParPAL2 / ParFLS2 & 92.9533 & 37.829 & 2.4572 & 0.0205 & \\
\hline \multicolumn{6}{|c|}{2020} \\
\hline Parameter & Estimation & Sd & $\mathbf{T}$ & P-value & $\mathbf{R} 2$ \\
\hline CONSTANT & 275.686 & 32.8475 & 8.39292 & 0 & 0.0784 \\
\hline ParFLS2 & 37.5785 & 22.0911 & 1.70107 & 0.0981 & \\
\hline \multicolumn{6}{|c|}{ 2019-2020 } \\
\hline Parameter & Estimation & Sd & $\mathbf{T}$ & P-value & $\mathbf{R 2}$ \\
\hline CONSTANT & 294.892 & 21.027 & 14.0244 & 0 & \\
\hline ParPAL2 / ParFLS1 & -18.5607 & 8.70188 & -2.13296 & 0.0366 & 0.063 \\
\hline
\end{tabular}

CHLOROGENIC ACID

\begin{tabular}{|c|c|c|c|c|}
\hline \multicolumn{5}{|c|}{2019} \\
\hline Parameter & Estimation & Sd & P-value & $\mathbf{R 2}$ \\
\hline CONSTANT & \multicolumn{2}{|c|}{290.90229 .67949 .80146} & $6 \quad 0$ & 0.293 \\
\hline ParDFR & \multicolumn{4}{|c|}{$-84.037122 .3848-3.754210 .0007$} \\
\hline \multicolumn{5}{|c|}{2020} \\
\hline Parameter & Estimation & Sd & P-value & $\mathbf{R 2}$ \\
\hline CONSTANT & \multicolumn{2}{|c|}{386.40739 .51989 .77757} & \multicolumn{2}{|c|}{00.0461} \\
\hline ParPAL1 & \multicolumn{2}{|c|}{$-28.677622 .355-1.28283$} & 30.2082 & 6 \\
\hline \multicolumn{5}{|c|}{ 2019-2020 } \\
\hline Parameter & Estimation & Sd & P-value & $\mathbf{R 2}$ \\
\hline CONSTANT & \multicolumn{2}{|c|}{318.27827 .134511 .7296} & \multicolumn{2}{|c|}{$\begin{array}{l}0 \\
0.0816\end{array}$} \\
\hline ParPAL2 / ParFLS1 & \multicolumn{4}{|c|}{$-27.404511 .2294-2.440430 .0173$} \\
\hline
\end{tabular}


CHLOROGENIC AND NEOCHLOROGENIC TOTAL CONTENT

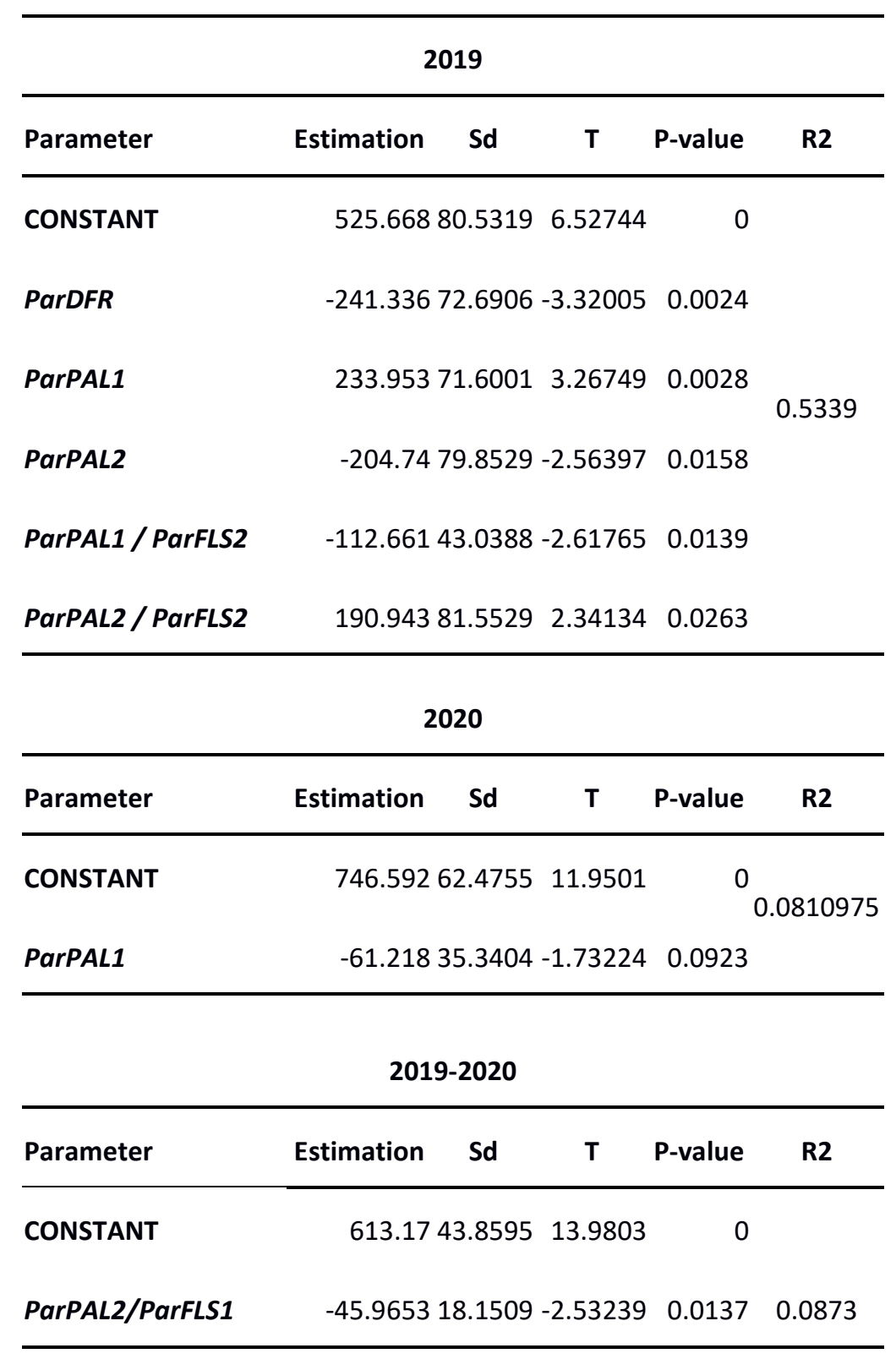


Table S18. Linear regression model in flavonols.

\begin{tabular}{|c|c|c|c|c|c|}
\hline \multicolumn{6}{|c|}{ RUTIN } \\
\hline \multicolumn{6}{|c|}{2019} \\
\hline Parameter & Estimatio & Sd & $\mathbf{T}$ & P-value & $\mathbf{R 2}$ \\
\hline CONSTANT & 562.793 & 90.0062 & 6.25283 & 0 & \\
\hline ParDFR & -294.562 & 78.952 & -3.7309 & 0.0009 & \\
\hline ParPAL1 & 251.179 & 86.265 & 2.91171 & 0.007 & \\
\hline ParPAL2 & -251.405 & 86.1836 & -2.91709 & 0.0069 & 0.366 \\
\hline ParPAL1 / ParPAL2 & -58.0325 & 26.0671 & -2.22627 & 0.0342 & \\
\hline ParPAL1 / ParFLS2 & -151.616 & 44.6125 & -3.39851 & 0.0021 & \\
\hline ParPAL2 / ParFLS2 & 304.215 & 84.1984 & 3.61308 & 0.0012 & \\
\hline \multicolumn{6}{|c|}{2020} \\
\hline Parameter & Estimatio & Sd & $\mathbf{T}$ & P-value & $\mathbf{R 2}$ \\
\hline CONSTANT & 211.629 & 21.3124 & 9.92987 & 0 & \\
\hline ParFLS1 / ParFLS2 & 9.19147 & 2.2127 & 4.15396 & 0.0003 & 0515868 \\
\hline ParPAL2 & 38.4111 & 8.62697 & 4.45244 & 0.0001 & . \\
\hline ParPAL1 / ParFLS2 & -2.59935 & 0.720061 & -3.6099 & 0.0011 & \\
\hline \multicolumn{6}{|c|}{$2019-2020$} \\
\hline Parameter & Estimatio & Sd & $\mathbf{T}$ & P-value & $\mathbf{R 2}$ \\
\hline CONSTANT & 356.984 & 23.5464 & 15.1609 & 0 & \multirow{2}{*}{0.0425} \\
\hline ParFLS1 & -19.4258 & 11.2636 & -1.72466 & 0.0892 & \\
\hline
\end{tabular}

\begin{tabular}{lrrrrrr}
\hline \multicolumn{2}{c}{ QUERCETIN_3-GLUCURONIDE } & & & \\
\hline & \multicolumn{2}{c}{ 2019 } & & & \\
\hline Parameter & Estimation & Sd & T & P-value & R2 \\
\hline CONSTANT & 48.7709 & 8.15762 & 5.97857 & 0 & \\
ParPAL2 & -23.0258 & 8.99826 & -2.55892 & 0.0156 & 0.1815 \\
ParPAL1 / ParFLS2 & -8.35646 & 3.44867 & -2.42309 & 0.0214 & \\
ParPAL2 / ParFLS2 & 17.4134 & 6.84919 & 2.5424 & 0.0162 & \\
\hline & & $\mathbf{2 0 2 0}$ & & & & \\
\hline Parameter & Estimation & Sd & T & P-value & R2 \\
CONSTANT & 28.3714 & 2.98273 & 9.51191 & & 0 & \\
ParFLS1 / ParFLS2 & 1.40385 & 0.294044 & 4.7743 & & 0 & 0.5852 \\
ParPAL1 / ParPAL2 & -4.4801 & 1.31522 & -3.40636 & 0.0019 & \\
ParPAL1 / ParFLS2 & -0.448444 & 0.0963081 & -4.65634 & 0.0001 & \\
\hline & & $\mathbf{2 0 1 9 - 2 0 2 0}$ & & & \\
\hline Parameter & Estimation & Sd & T & P-value & R2 \\
\hline CONSTANT & 32.7225 & 3.31507 & 9.87083 & & \multirow{2}{*}{0.04786} \\
ParPAL1 / ParPAL2 & -2.83612 & 1.52274 & -1.8625 & 0.0668 & \\
\hline
\end{tabular}


RUTIN+QUERCETIN-3-GLUCURONIDE

\begin{tabular}{|c|c|c|c|c|c|}
\hline \multicolumn{6}{|c|}{2019} \\
\hline Parameter & Estimation & Sd & $\mathbf{T}$ & P-value & $\mathbf{R 2}$ \\
\hline CONSTANT & 615.21 & 98.5015 & 6.24569 & 0 & \\
\hline ParDFR & -313.956 & 86.4039 & -3.63358 & 0.0011 & \\
\hline ParPAL1 & 282.717 & 94.4072 & 2.99465 & 0.0057 & \\
\hline ParPAL2 & -293.832 & 94.3181 & -3.11533 & 0.0042 & 0.368 \\
\hline ParPAL1 / ParPAL2 & -64.1542 & 28.5275 & -2.24885 & 0.0326 & \\
\hline ParPAL1 / ParFLS2 & -172.127 & 48.8232 & -3.52552 & 0.0015 & \\
\hline ParPAL2 / FLS2 & 341.894 & 92.1456 & 3.71037 & 0.0009 & \\
\hline \multicolumn{6}{|c|}{2020} \\
\hline Parameter & Estimation & Sd & $\mathbf{T}$ & P-value & $\mathbf{R 2}$ \\
\hline CONSTANT & 227.956 & 23.062 & 9.88451 & 0 & \\
\hline ParFLS1 /Par FLS2 & 10.8076 & 2.39435 & 4.51382 & 0.0001 & 0.536877 \\
\hline ParPAL2 & 41.3101 & 9.33517 & 4.42521 & 0.0001 & \\
\hline ParPAL1 / ParFLS2 & -3.12702 & 0.779172 & -4.01326 & 0.0004 & \\
\hline \multicolumn{6}{|c|}{$2019-2020$} \\
\hline Parameter & Estimation & Sd & $\mathbf{T}$ & P-value & $\mathbf{R 2}$ \\
\hline CONSTANT & 387.332 & 25.922 & 14.9423 & 0 & 0.0417 \\
\hline ParFLS1 & -21.1966 & 12.3999 & -1.70941 & 0.092 & \\
\hline
\end{tabular}


ANNEX III. Supplementary Documents 
Document S1. Acceptance mail of the publication "Nutraceutical profiles of apricots (Prunus armeniaca L.) as a source of fruit quality traits for breeding" (Chapter 1) in the Spanish Journal of Agricultural Research.

De SJAR <publinia@inia.es> $\lesssim$

\section{Asunto SJAR [18331] Editor Decision}

Responder a María Roca <mroca@ig.csic.es> §

A garcia_zur@gva.es $₫$

Cc Carmen De Blas < sjar@inia.es> $\lesssim$, Elena Prats <elena.prats@ias.csic.es> $\lesssim$

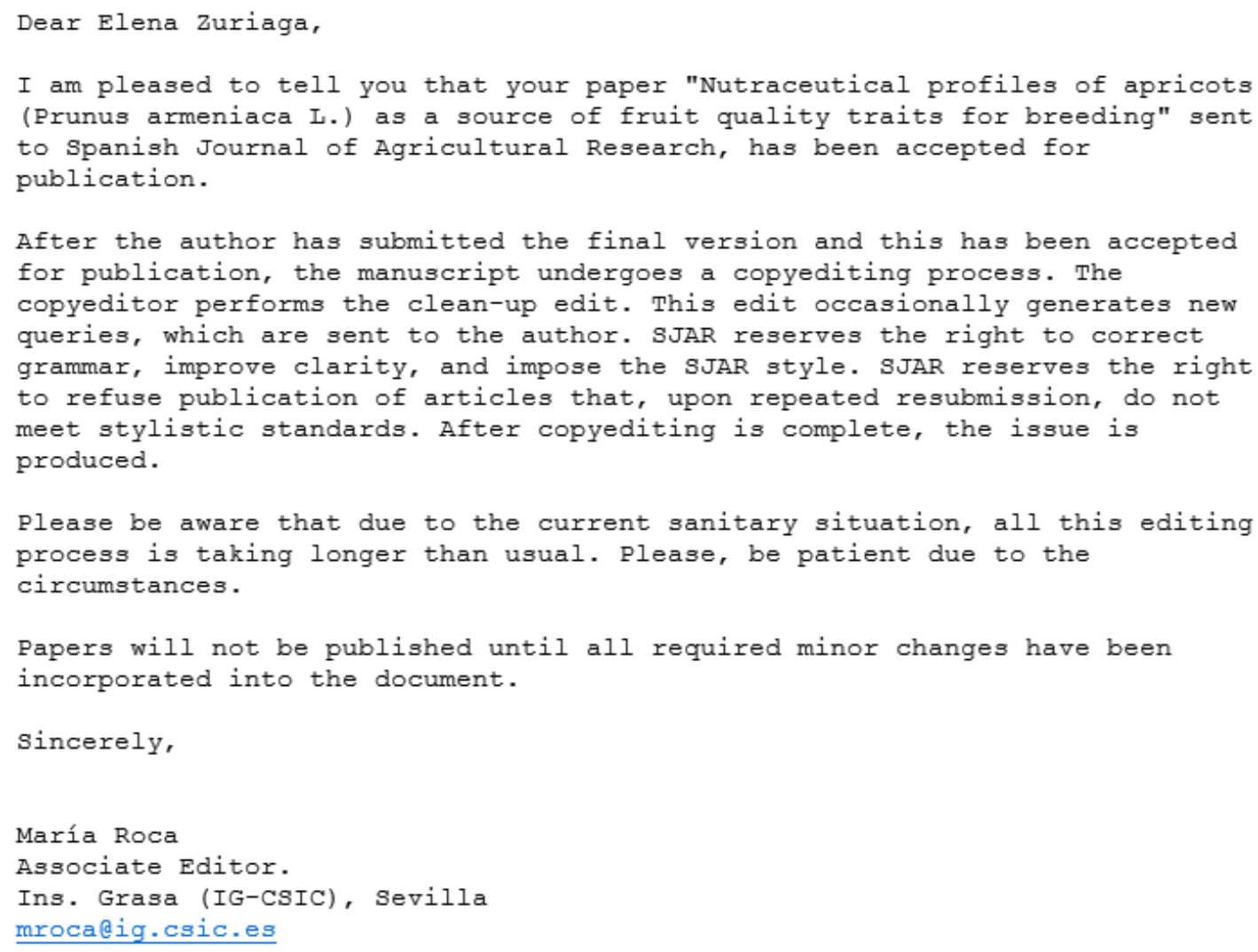

